\title{
Fluctuation Phenomena in Superconductors
}

\author{
Anatoly Larkin and Andrei Varlamov
}

To our friend Lev Aslamazov, in memoriam

\section{Contents}

\section{Introduction}

2.Ginzburg-Landau formalism. Thermodynamics.

2.1 Fluctuation contribution to heat capacity

2.1.1 GL functional.

2.1.2 Zero dimensionality: the exact solution.

2.1.3 Arbitrary dimensionality: case $T \geq T_{c}$.

2.1.4 Arbitrary dimensionality: case $T<T_{c}$.

2.2 Ginzburg-Levanyuk criterion

2.3 Scaling and renormalization group

2.4 Fluctuation diamagnetism

2.4.1 Qualitative preliminaries.

2.4.2 Zero-dimensional diamagnetic susceptibility.

2.4.3 GL treatment of fluctuation magnetization.

3. Fluctuations below critical temperature (to be published in the complete version)

3. Ginzburg-Landau theory of fluctuations in transport phenomena

3.1 Time dependent GL equation

3.2 Paraconductivity

3.3 General expression for paraconductivity

3.4 Fluctuation conductivity of layered superconductor

3.4.1 In-plane conductivity.

3.4.2 Out-of plane conductivity.

3.4.3 Analysis of the general expressions.

3.5 Magnetic field angular dependence of paraconductivity

4. Fluctuations near superconductor-insulator transition

4.1 Quantum phase transition

$4.23 \mathrm{D}$ superconductors

$4.32 \mathrm{D}$ superconductors

4.3.1 Preliminaries.

4.3.2 Boson mechanism of the $T_{c}$ suppression.

4.3.3 Fermion mechanism of $T_{c}$ suppression. 


\section{Microscopic derivation of the Time-Dependent Ginzburg-Landau equation}

5.1 Preliminaries

5.2 The Cooper channel of electron-electron interaction

5.3 Superconductor with impurities

5.3.1 Account for impurities.

5.3.2 Propagator.

5.4. Microscopic theory of fluctuation conductivity of layered superconductor

5.4.1 Qualitative discussion of different fluctuation contributions

5.4.2 Generalities

5.4.3 Aslamazov-Larkin contribution

5.4.4 Contributions from fluctuations of the density of states

5.4.5 Maki-Thompson contribution

5.4.6 Discussion

6. Manifestation of fluctuations in various properties

6.1 The effects of fluctuations on magnetoconductivity

6.2 Fluctuations far from $T_{c}$ or in strong magnetic fields

6.2.1 Fluctuation magnetic susceptibility far from transition.

6.2.2 Fluctuation magnetoconductivity far from transition

6.2.3 Fluctuations in magnetic fields near $H_{c 2}(0)$

6.3 The effect of fluctuations on the Hall conductivity

6.4 Fluctuations in the ultra-clean case

6.5 The effect of fluctuations on the one-electron density of states and on tunneling measurements

6.5.1 Density of states

6.5.2 The effect of fluctuations on the tunnel current

6.6 Nonlinear fluctuation effects

6.7 The effect of fluctuation on the optical conductivity

6.8 Thermoelectric power above the superconducting transition

6.9 The effect of fluctuations on NMR characteristics

6.9.1 Preliminaries.

6.9.2 Spin Susceptibility.

6.9.3 Relaxation Rate.

7. Conclusions

8. Acknowledgments

9. Bibliography

\section{Introduction}

A major success of low temperature physics was achieved with the introduction by Landau of the notion of quasiparticles. According to his hypothesis, the properties of many body interacting systems at low temperatures are determined by the spectrum of some low energy, long living excitations 
(quasiparticles). Another milestone of many body theory is the Mean Field Approximation (MFA), which permitted achieving considerable progress in the theory of phase transitions. Phenomena which cannot be described by the quasiparticle method or by MFA are usually called fluctuations. The BCS theory of superconductivity is a bright example of the use of both the quasiparticle description and MFA. The success of the BCS theory for traditional superconductors was determined by the fact that fluctuations give small corrections with respect to the MFA results.

During the first half of the century after the discovery of superconductivity the problem of fluctuation smearing of the superconducting transition was not even considered. In bulk samples of traditional superconductors the critical temperature $T_{c}$ sharply divides the superconducting and the normal phases. It is worth mentioning that such behavior of the physical characteristics of superconductors is in perfect agreement both with the GinzburgLandau (GL) phenomenological theory (1950) [1] and the BCS microscopic theory of superconductivity (1957) [2].

The characteristics of high temperature and organic superconductors, low dimensional and amorphous superconducting systems studied today, strongly differ from those of the traditional superconductors discussed in textbooks. The transitions turn out to be much more smeared out. The appearance of superconducting fluctuations above critical temperature leads to precursor effects of the superconducting phase occurring while the system is still in the normal phase, sometimes far from $T_{c}$. The conductivity, the heat capacity, the diamagnetic susceptibility, the sound attenuation, etc. may increase considerably in the vicinity of the transition temperature.

The first numerical estimation of the fluctuation contribution to the heat capacity of a superconductor in the vicinity of $T_{c}$ was done by Ginzburg in 1960 [3]. In that paper he showed that superconducting fluctuations increase the heat capacity even above $T_{c}$. In this way fluctuations change the temperature dependence of the specific heat in the vicinity of critical temperature, where, in accordance with the phenomenological GL theory of second order phase transitions, a jump should take place. The range of temperatures where the fluctuation correction to the heat capacity of a bulk clean conventional superconductor is relevant was estimated by Ginzburg 1 to be

$$
G i=\frac{\delta T}{T_{c}} \sim\left(\frac{T_{c}}{E_{F}}\right)^{4} \sim 10^{-12} \div 10^{-14}
$$

where $E_{F}$ is the Fermi energy. The correction occurs in a temperature range $\delta T$ many orders of magnitude smaller than that accessible in real experiments.

\footnotetext{
1 The expression for the width of the strong fluctuation region in terms of the Landau phenomenological theory of phase transitions was obtained by Levanyuk 㭡. So in the modern theory of phase transitions the relative temperature width of fluctuation region is called the Ginzburg-Levanyuk parameter $G i_{(D)}$, where $D$ is the effective space dimensionality.
} 
In the 1950s and 60s the formulation of the microscopic theory of superconductivity, the theory of type-II superconductors and the search for high- $T_{c}$ superconductivity attracted the attention of researchers to dirty systems, superconducting films and filaments. In 1968, in the papers of L. G. Aslamazov and A. I. Larkin [5], K.Maki [6] and a little later in the paper of R.S.Thompson [7] the fundaments of the microscopic theory of fluctuations in the normal phase of a superconductor in the vicinity of the critical temperature were formulated. This microscopic approach confirmed Ginzburg's evaluation [3] for the width of the fluctuation region in a bulk clean superconductor. Moreover, was found that the fluctuation effects increase drastically in thin dirty superconducting films and whiskers. In the cited papers was demonstrated that fluctuations affect not only the thermodynamical properties of superconductor but its dynamics too. Simultaneously the fluctuation smearing of the resistive transition in bismuth amorphous films was experimentally found by Glover [8], and it was perfectly fitted by the microscopic theory.

In the BCS theory [2] only the Cooper pairs forming a Bose-condensate are considered. Fluctuation theory deals with the Cooper pairs out of the condensate. In some phenomena these fluctuation Cooper pairs behave similarly to quasiparticles but with one important difference. While for the well defined quasiparticle the energy has to be much larger than its inverse life time, for the fluctuation Cooper pairs the "binding energy" $E_{0}$ turns out to be of the same order. The Cooper pair life time $\tau_{G L}$ is determined by its decay into two free electrons. Evidently at the transition temperature the Cooper pairs start to condense and $\tau_{G L}=\infty$. So it is natural to suppose from dimensional analysis that $\tau_{G L} \sim \hbar / k_{B}\left(T-T_{c}\right)$. The microscopic theory confirms this hypothesis and gives the exact coefficient:

$$
\tau_{G L}=\frac{\pi \hbar}{8 k_{B}\left(T-T_{c}\right)} .
$$

Another important difference of the fluctuation Cooper pairs from quasiparticles lies in their large size $\xi(T)$. This size is determined by the distance on which the electrons forming the fluctuation Cooper pair move away during the pair life-time $\tau_{G L}$. In the case of an impure superconductor the electron motion is diffusive with the diffusion coefficient $\mathcal{D} \sim v_{F}^{2} \tau(\tau$ is the electron scattering time民, and $\xi_{d}(T)=\sqrt{\mathcal{D} \tau_{G L}} \sim v_{F} \sqrt{\tau \tau_{G L}}$. In the case of a clean superconductor, where $k_{B} T \tau \gg \hbar$, impurity scattering does not affect any more the electron correlations. In this case the time of electron ballistic motion turns out to be less than the electron-impurity scattering time $\tau$ and is determined by the uncertainty principle: $\tau_{b a l} \sim \hbar / k_{B} T$. Then this time has

\footnotetext{
${ }^{2}$ Strictly speaking $\tau$ in the most part of future results should be understood as the electron transport scattering time $\tau_{t r}$. Nevertheless, as it is well known, in the case of isotropic scattering these values coincide, so for sake of simplicity we will use hereafter the symbol $\tau$.
} 
to be used in this case for determination of the effective size instead of $\tau$ : $\xi_{c}(T) \sim v_{F} \sqrt{\hbar \tau_{G L} / k_{B} T}$. In both cases the coherence length grows with the approach to the critical temperature as $\left(T-T_{c}\right)^{-1 / 2}$, and we will write it down in the unique way $\left(\xi=\xi_{c, d}\right)$ :

$$
\xi(T)=\frac{\xi}{\sqrt{\epsilon}}, \quad \epsilon=\frac{T-T_{c}}{T_{c}} .
$$

The microscopic theory in the case of an isotropic Fermi surface gives for $\xi$ the precise expression:

$$
\xi_{(D)}^{2}=-\frac{v_{F}^{2} \tau^{2}}{D}\left\{\psi\left(\frac{1}{2}+\frac{\hbar}{4 \pi k_{B} T \tau}\right)-\psi\left(\frac{1}{2}\right)-\frac{\hbar}{4 \pi k_{B} T \tau} \psi^{\prime}\left(\frac{1}{2}\right)\right\}
$$

where $\psi(x)$ is the digamma function and $D=3,2,1$ is the space dimensionality. In the clean $(c)$ and dirty $(d)$ limits:

$$
\begin{aligned}
& \xi_{c}=0.133 \frac{\hbar v_{F}}{k_{B} T_{c}} \sqrt{\frac{3}{D}}=0.74 \xi_{0} \sqrt{\frac{3}{D}}, \\
& \xi_{d}=0.36 \sqrt{\frac{\hbar v_{F} l}{k_{B} T_{c}} \frac{3}{D}}=0.85 \sqrt{\xi_{0} l} \sqrt{\frac{3}{D}} .
\end{aligned}
$$

Here $l=v_{F} \tau$ is the electron mean free path and $\xi_{0}=\hbar v_{F} / \pi \Delta(0)$ is the conventional BCS definition of the coherence length of a clean superconductor at zero temperature. One can see that (5) and (6) coincide with the above estimations 3 .

Finally it is necessary to recognize that fluctuation Cooper pairs can be really treated as classical objects, but these objects instead of Boltzmann particles appear as classical fields in the sense of Rayleigh-Jeans. This means that in the general Bose-Einstein distribution function only small energies $E(p)$ are involved and the exponent can be expanded:

$$
n(p)=\frac{1}{\exp \left(\frac{E(p)}{k_{B} T}\right)-1}=\frac{k_{B} T}{E(p)} .
$$

${ }^{3}$ Let us stress some small numerical difference between our Exp. (4) and the usual definition of the coherence length. We are dealing near the critical temperature, so the definition (4) is natural and permits us to avoid many numerical coefficients in further calculations. The cited coherence length $\xi_{0}=\hbar v_{F} / \pi \Delta(0)=$ $0.18 \hbar v_{F} / k_{B} T_{c}$, as is evident, was introduced for zero temperature and an isotropic $3 D$ superconductor.

It is convenient to determine the coherence length also from the formula for the upper critical field: $H_{c 2}(T)=A(T) \Phi_{0} / 2 \pi \xi^{2}(T) . A\left(T_{c}\right)=1$, while its value at $T=0$ depends on the impurities concentration. For the dirty case the appropriate value was found by K.Maki 9] $A_{d}(0)=0.69$, for the clean case by L.Gor'kov [10] $A_{c}^{2 D}(0)=0.59, A_{c}^{3 D}(0)=0.72$. 
This is why the more appropriate tool to study fluctuation phenomena is not the Boltzmann transport equation but the GL equation for classical fields. Nevertheless at the qualitative level the treatment of fluctuation Cooper pairs as particles with the density $n_{(D)}=\int n(p) \frac{d^{D} p}{(2 \pi \hbar)^{D}}$ often turns out to be useful 同.

Below will be demonstrated both in the framework of the phenomenological Ginzburg-Landau theory and the microscopic BCS theory that in the vicinity of the transition

$$
E(p)=\alpha k_{B}\left(T-T_{c}\right)+\frac{\mathbf{p}^{2}}{2 m^{*}}=\frac{1}{2 m^{*}}\left[\hbar^{2} / \xi^{2}(T)+\mathbf{p}^{2}\right] .
$$

Far from transition temperature the dependence $n(p)$ turns out to be more sophisticated than (耳), nevertheless one can always write it in the form

$$
n(p)=\frac{m^{*} k_{B} T}{\hbar^{2}} \xi^{2}(T) f\left(\frac{\xi(T) p}{\hbar}\right) .
$$

In classical field theory the notions of the particle distribution function $n(p)$ (proportional to $E^{-1}(p)$ in our case) and Cooper pair mass $m^{*}$ are poorly determined. At the same time the characteristic value of the Copper pair center of mass momentum can be defined and it turns out to be of the order of $p_{0} \sim \hbar / \xi(T)$. So for the combination $m^{*} E\left(p_{0}\right)$ one can write $m^{*} E\left(p_{0}\right) \sim p_{0}^{2} \sim \hbar^{2} / \xi^{2}(T)$. In fact the particles density enters into many physical values in the combination $n / m^{*}$. As the consequence of the above observation it can be expressed in terms of the coherence length:

$$
\frac{n_{(D)}}{m^{*}}=\frac{k_{B} T}{m^{*} E\left(p_{0}\right)}\left(\frac{p_{0}}{\hbar}\right)^{D} \sim \frac{k_{B} T}{\hbar^{2}} \xi^{2-D}(T),
$$

$p_{0}^{D}$ here estimates the result of momentum integration.

For example we can evaluate the fluctuation Cooper pairs contribution to conductivity by using the Drude formula

$$
\sigma=\frac{n e^{2} \tau}{m^{*}} \Rightarrow \frac{k_{B} T}{\hbar^{2}} d^{D-3} \xi^{2-D}(T)(2 e)^{2} \tau_{G L}(\epsilon) \sim \epsilon^{D / 2-2} .
$$

\footnotetext{
${ }^{4}$ This particle density is defined in the $(D)$-dimensional space. This means that it determines the normal volume density of pairs in $3 D$ case, the density per square unit in $2 D$ case and the number of pairs per unit length in $1 D$. The real three dimensional density $n$ can be defined too: $n=d^{D-3} n_{(D)}$, where $d$ is the thickness of the film or wire.
} 
Analogously a qualitative understanding of the increase in the diamagnetic susceptibility above the critical temperature may be obtained from the wellknown Langevin expression for the atomic susceptibilityp:

$$
\chi=-\frac{e^{2}}{c^{2}} \frac{n}{m^{*}}\left\langle R^{2}\right\rangle \Rightarrow-\frac{4 e^{2}}{c^{2}} \frac{k_{B} T}{\hbar^{2}} d^{D-3} \xi^{4-D}(T) \sim-\epsilon^{D / 2-2} .
$$

Besides these examples of the direct influence of fluctuations on superconducting properties, indirect manifestations by means of quantum interference in the pairing process and of renormalization of the density of one-electron states in the normal phase of superconductor take place. These effects, being much more sophisticated, have a purely quantum nature, and in contrast to the direct Cooper pair contributions require microscopic consideration. This is why, in developing phenomenological methods through the first five Sections of this review, we will deal with the direct fluctuation pair contributions only. The sixth Section is devoted to the microscopic justification of the time dependent Ginzburg-Landau equation. The description of the microscopic theory of fluctuations, including indirect fluctuation effects, discussion of their manifestations in various physical properties of superconductors will be given in the Sections 7-8.

The first seven Sections are written in detail, so they can serve as a textbook. On the contrary, in the last (eighth) Section a wide panorama of fluctuation effects in different physical properties of superconductors is presented. Thus this Section has more of a handbook character, and the intermediate calculations often are omitted.

Finally we would like to mention that the number of articles devoted to superconducting fluctuations published in the last 33 years is of the order of ten thousands, so our bibliography list does not pretend nor at the completeness nor at the establishment of the rigorous priorities.

\section{Ginzburg-Landau formalism. Thermodynamics}

\subsection{Fluctuation contribution to heat capacity}

GL functional. The complete description of the thermodynamic properties of a system can be done through the exact calculation of the partition function $[$ :

$$
Z=\operatorname{Tr}\left\{\exp \left(-\frac{\widehat{\mathcal{H}}}{T}\right)\right\} .
$$

${ }^{5}$ This formula is valid for the dimensionalities $D=2,3$, when fluctuation Cooper pair has the possibility to "rotate" in the applied magnetic field and the average square of the rotation radius is $<R^{2}>\sim \xi^{2}(T)$. "Size" effects, important for low dimensional samples, will be discussed later on.

${ }^{6}$ Hereafter $\hbar=k_{B}=c=1$. 
As discussed in the Introduction, in the vicinity of the superconducting transition, side by side with the fermionic electron states, fluctuation Cooper pairs of a bosonic nature appear in the system. As already mentioned, they can be described by means of classical bosonic fields $\Psi(\mathbf{r})$ which can be treated as "Cooper pair wave functions ". So the calculation of the trace in (13) can be separated into a summation over the "fast" electron degrees of freedom and a further functional integration carried out over all possible configurations of the "slow" Cooper pairs wave functions:

$$
Z=\int D \Psi(\mathbf{r}) \mathcal{Z}[\Psi(\mathbf{r})]
$$

where

$$
\mathcal{Z}[\Psi(\mathbf{r})]=\exp \left(-\frac{\mathcal{F}[\Psi(\mathbf{r})]}{T}\right)
$$

is the system partition function in a fixed bosonic field $\Psi(\mathbf{r})$, already summed over the electronic degrees of freedom. Here it is assumed that the classical field dependent part of the Hamiltonian can be chosen in the spirit of the GL approach :

$$
F[\Psi(r)]=F_{N}+\int d V\left\{a|\Psi(\mathbf{r})|^{2}+\frac{b}{2}|\Psi(\mathbf{r})|^{4}+\frac{1}{4 m}|\nabla \Psi(\mathbf{r})|^{2}\right\} . .
$$

Let us discuss the coefficients of this functional. In accordance with the Landau hypothesis, the coefficient $a$ goes to zero at the transition point and depends linearly on $T-T_{c}$. Then $a=\alpha T_{c} \epsilon$; all the coefficients $\alpha, b$ and $m$ are supposed to be positive and temperature independent. Concerning the magnitude of the coefficients it is necessary to make the following comment. One of these coefficients can always be chosen arbitrary: this option is related to the arbitrariness of the Cooper pair wave function normalization. Nevertheless the product of two of them is fixed by dimensional analysis: $m a \sim \xi^{-2}(T)$. Another combination of the coefficients, independent of the wave function normalization and temperature, is $\alpha^{2} / b$. One can see that it has the dimensionality of the density of states. Since these coefficients were obtained by a summation over the electronic degrees of freedom, the only reasonable candidate for this value is the one electron density of states $\nu$. The microscopic theory gives the precise coefficients for the above relations:

$$
4 m \alpha T_{c}=\xi^{-2} ; \alpha^{2} / b=\frac{8 \pi^{2}}{7 \zeta(3)} \nu,
$$

where $\zeta(x)$ is the Riemann zeta function, $\zeta(3)=1.202$. One can notice that the arbitrariness in the normalization of the order parameter amplitude leads

\footnotetext{
${ }^{7}$ For simplicity the magnetic field is assumed to be zero.
} 
to the unambiguity in the choice of the Cooper mass introduced in (16) as $2 m$. Indeed, this value enters in (17) in the product with $\alpha$ so one of these parameters has to be fixed. In the case of a clean D-dimensional superconductor it is natural to suppose that the Copper pair mass is equal to two free electron masses what results in

$$
\alpha_{(D)}=\frac{2 D \pi^{2}}{7 \zeta(3)} \frac{T_{c}}{E_{F}} .
$$

As the first step in the Landau theory of phase transitions $\Psi$ is supposed to be independent of position. This assumption in the limit of sufficiently large volume $V$ of the system permits a calculation of the functional integral in (14) by the method of steepest descent. Its saddle point determines the equilibrium value of the order parameter

$$
|\widetilde{\Psi}|^{2}=\left\{\begin{array}{l}
-\alpha T_{c} \epsilon / b, \epsilon<0 \\
0, \epsilon>0
\end{array} .\right.
$$

Choosing $\alpha$ in accordance with (18) one finds that this value coincides with the superfluid density $n_{s}$ of the microscopic theory [2].

The fluctuation part of the free energy related to the transition is determined by the minimum of the functional (16):

$$
F=(\mathcal{F}[\Psi])_{\min }=\mathcal{F}[\widetilde{\Psi}]=\left\{\begin{array}{l}
F_{N}-\frac{\alpha^{2} T_{c}^{2} \epsilon^{2}}{2 b} V, \epsilon<0 \\
F_{N}, \epsilon>0
\end{array} .\right.
$$

From the second derivative of (20) one can find an expression for the jump of the specific heat capacity at the phase transition point:

$$
\begin{aligned}
\Delta C & =C_{S}-C_{N}=\frac{T_{c}}{V}\left(\frac{\partial S_{S}}{\partial T}\right)-\frac{T_{c}}{V}\left(\frac{\partial S_{N}}{\partial T}\right)= \\
& =-\frac{1}{V T_{c}}\left(\frac{\partial^{2} F}{\partial \epsilon^{2}}\right)=\frac{\alpha^{2}}{b} T_{c}=\frac{8 \pi^{2}}{7 \zeta(3)} \nu T_{c} .
\end{aligned}
$$

Let us mention that the jump of the heat capacity was obtained because of the system volume was taken to infinity first, and after this the reduced temperature $\epsilon$ was set equal to zero.

Zero dimensionality: the exact solution. In a system of finite volume fluctuations smear out the jump in heat capacity. For a small superconducting sample with the characteristic size $d \ll \xi(T)$ the space independent mode $\Psi_{0}=\Psi \sqrt{V}$ defines the main contribution to the free energy: 


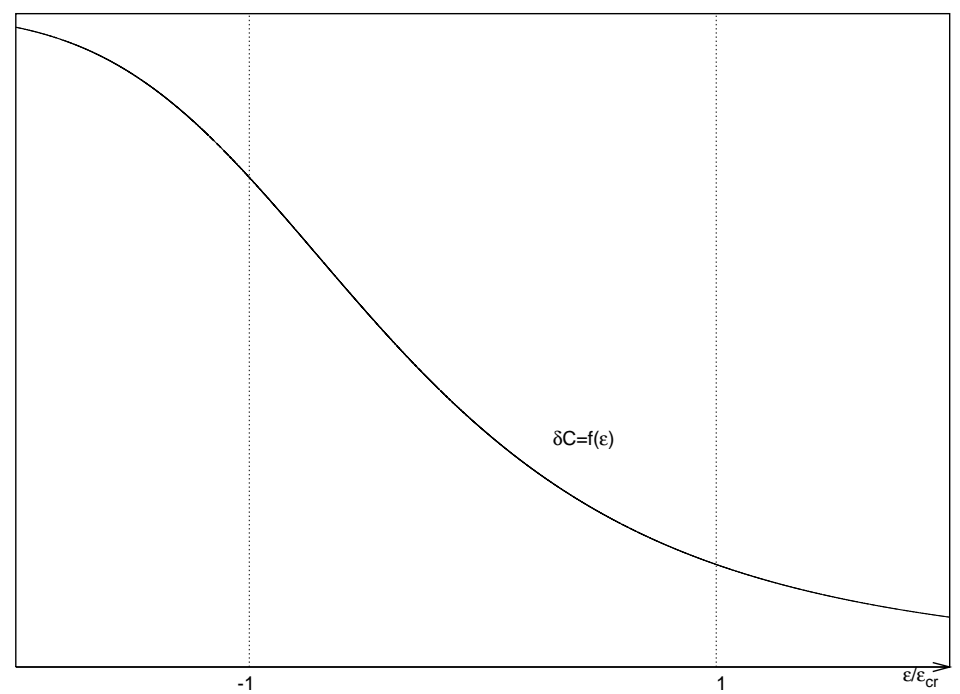

Fig. 1. Temperature dependence of the heat capacity of superconducting grains in the region of the critical temperature

$$
\begin{aligned}
Z_{(0)} & =\int d^{2} \Psi_{0} \mathcal{F}\left[\Psi_{0}\right]=\pi \int d\left|\Psi_{0}\right|^{2} \exp \left(-\frac{\left(a\left|\Psi_{0}\right|^{2}+\frac{b}{2 V}\left|\Psi_{0}\right|^{4}\right)}{T}\right)= \\
& =\left.\sqrt{\frac{\pi^{3} V T}{2 b}} \exp \left(x^{2}\right)(1-\operatorname{erf}(x))\right|_{x=a \sqrt{\frac{V}{2 b T}}}
\end{aligned}
$$

By evaluating the second derivative of this exact result [11] one can find the temperature dependence of the superconducting granular heat capacity (see Fig. 1). It is evident that the smearing of the jump takes place in the region of temperatures in the vicinity of transition where $x \sim 1$, i.e.

$$
\epsilon_{c r}=G i_{(0)}=\frac{\sqrt{7 \zeta(3)}}{2 \pi} \frac{1}{\sqrt{\nu T_{c} V}} \approx 13,3\left(\frac{T_{c 0}}{E_{F}}\right) \sqrt{\frac{\xi_{0}^{3}}{V}}
$$

where we have supposed that the granule is clean; $T_{c 0}$ and $\xi_{0}$ are the critical temperature and the zero temperature coherence length (see the footnote 3 in Introduction) of the appropriate bulk material. From this formula one can see that even for granule with the size $d \sim \xi_{0}$ the smearing of the transition still is very narrow.

Far above the critical region, where $G i_{(0)} \ll \epsilon \ll 1$, one can use the asymptotic expression for the $\operatorname{erf}(x)$ function and find

$$
F_{(0)}=-T \ln Z_{(0)}=-T \ln \frac{\pi}{\alpha \epsilon} .
$$


Calculation of the second derivative gives an expression for the fluctuation part of the heat capacity in this region:

$$
\delta C_{(0)}=1 / \epsilon^{2} .
$$

The experimental study of the heat capacity of small Sn particles in the vicinity of transition was done in $[12$

Arbitrary dimensionality: case $\boldsymbol{T} \geq \boldsymbol{T}_{\boldsymbol{c}}$. It is possible to estimate the fluctuation contribution to the heat capacity for a specimen of an arbitrary effective dimensionality on the basis of the following observation. The volume of the specimen may be divided into regions of size $\xi(T)$, which are weakly correlated with each other. Then the whole free energy can be estimated as the free energy of one such zero-dimensional specimen (23), multiplied by their number $N_{(D)}=V \xi^{-D}(T)$ :

$$
F_{(D)}=-T V \xi^{-D}(T) \ln \frac{\pi}{\alpha \epsilon} .
$$

This formula gives the correct temperature dependence for the free energy for even dimensionalities. A more accurate treatment removes the $\ln \epsilon$ dependence from it in the case of the odd dimensions.

Let us begin with the calculation of the fluctuation contribution to the heat capacity in the normal phase of a superconductor. We restrict ourselves to the region of temperatures beyond the immediate vicinity of transition, where this correction is still small. In this region one can omit the fourth order term in $\Psi(\mathbf{r})$ with respect to the quadratic one and write down the GL functional, expanding the order parameter in a Fourier series:

$$
F\left[\Psi_{\mathbf{k}}\right]=F_{N}+\sum_{\mathbf{k}}\left[a+\frac{\mathbf{k}^{2}}{4 m}\right]\left|\Psi_{\mathbf{k}}\right|^{2}=\alpha T_{c} \sum_{\mathbf{k}}\left(\epsilon+\xi^{2} \mathbf{k}^{2}\right)\left|\Psi_{\mathbf{k}}\right|^{2} .
$$

Here $\Psi_{\mathbf{k}}=\frac{1}{\sqrt{V}} \int \Psi(\mathbf{r}) e^{-i \mathbf{k r}} d V$ and the summation is carried out over the vectors of the reciprocal space. Now we see that the free energy functional appears as a sum of energies of the independent modes $\mathbf{k}$. The functional integral for the partition function (15) can be separated to a product of Gaussian type integrals over these modes:

$$
Z=\prod_{\mathbf{k}} \int d^{2} \Psi_{\mathbf{k}} \exp \left\{-\alpha\left(\epsilon+\frac{\mathbf{k}^{2}}{4 m \alpha T_{c}}\right)\left|\Psi_{\mathbf{k}}\right|^{2}\right\}
$$

Carrying out these integrals, one gets the fluctuation contribution to the free energy:

$$
F(\epsilon>0)=-T \ln Z=-T \sum_{\mathbf{k}} \ln \frac{\pi}{\alpha\left(\epsilon+\frac{\mathbf{k}^{2}}{4 m \alpha T_{c}}\right)} .
$$


The appropriate correction to the specific heat capacity of a superconductor at temperatures above the critical temperature may thus be calculated. We are interested in the most singular term in $\epsilon^{-1}$, so the differentiation over the temperature can be again replaced by that over $\epsilon$ :

$$
\delta C_{+}=-\frac{1}{V T_{c}}\left(\frac{\partial^{2} F}{\partial \epsilon^{2}}\right)=\frac{1}{V} \sum_{\mathbf{k}} \frac{1}{\left(\epsilon+\frac{\mathbf{k}^{2}}{4 m \alpha T_{c}}\right)^{2}} .
$$

The result of the summation over $\mathbf{k}$ strongly depends on the linear sizes of the sample, i.e. on its effective dimensionality. As it is clear from (29), the scale with which one has to compare these sizes is determined by the value $\left(4 m \alpha T_{c} \epsilon\right)^{-\frac{1}{2}}$ which, as was already mentioned above, coincides with the effective size of Cooper pair $\xi(T)$. Thus, if all dimensions of the sample considerably exceed $\xi(T)$, one can integrate over $(2 \pi)^{-3} L_{x} L_{y} L_{z} d k_{x} d k_{y} d k_{z}$ instead of summing over $n_{x}, n_{y}, n_{z}$. In the case of arbitrary dimensionality the fluctuation correction to the heat capacity turns out to be

$$
\delta C_{+}=\frac{V_{D}}{V} \int \frac{1}{\left(\epsilon+\frac{\mathbf{k}^{2}}{4 m \alpha T_{c}}\right)^{2}} \frac{d^{D} \mathbf{k}}{(2 \pi)^{D}}=\vartheta_{D} \frac{V_{D}}{V} \frac{\left(4 m \alpha T_{c}\right)^{\frac{D}{2}}}{\epsilon^{2-\frac{D}{2}}}
$$

where $V_{D}=V, S, L, 1$ for $D=3,2,1,0$. For the coefficients $\vartheta_{D}$ it is convenient to write an expression valid for an arbitrary dimensionality $D$, including fractional ones. For a space of fractional dimensionality we just mention that the momentum integration in spherical coordinates is carried out according to the rule: $\int d^{D} \mathbf{k} /(2 \pi)^{D}=\mu_{D} \int k^{D-1} d k$, where

$$
\mu_{D}=\frac{D}{2^{D} \pi^{D / 2} \Gamma(D / 2+1)}
$$

and $\Gamma(x)$ is a gamma-function. The coefficient in (30) can be expressed in terms of the gamma-function too:

$$
\vartheta_{D}=\frac{\Gamma(2-D / 2)}{2^{D} \pi^{D / 2}}
$$

yielding $\vartheta_{1}=1 / 4, \vartheta_{2}=1 / 4 \pi$ and $\vartheta_{3}=1 / 8 \pi$.

In the case of small particles with characteristic sizes $d \lesssim \xi(\epsilon)$ the appropriate fluctuation contribution to the free energy and the specific heat capacity coincides with the asymptotics of the exact results (23) and (24). From the formula given above it is easy to see that the role of fluctuations increases when the effective dimensionality of the sample or the electron mean free path decrease. 
Arbitrary dimensionality: case $\boldsymbol{T}<\boldsymbol{T}_{\boldsymbol{c}}$. The general expressions (14) and $(16)$ allow one to find the fluctuation contribution to heat capacity below $T_{c}$. For this purpose let us restrict ourselves to the region of temperatures not very close to $T_{c}$ from below, where fluctuations are sufficiently weak. In this case the order parameter can be written as the sum of the equilibrium $\widetilde{\Psi}($ see $(19))$ and fluctuation $\psi(\mathbf{r})$ parts:

$$
\Psi(\mathbf{r})=\widetilde{\Psi}+\psi(\mathbf{r}) .
$$

Keeping in (16) the terms up to the second order in $\psi(\mathbf{r})$ and up to the fourth order in $\widetilde{\Psi}$, one can find

$$
\begin{aligned}
\mathcal{Z}[\widetilde{\Psi}]= & \exp \left(-\frac{a \widetilde{\Psi}^{2}+b / 2 \widetilde{\Psi}^{4}}{T}\right) \prod_{\mathbf{k}} \int d \operatorname{Re} \psi_{\mathbf{k}} d \operatorname{Im} \psi_{\mathbf{k}} \times \\
& \times \exp \left\{-\frac{1}{T}\left[\left(3 b \widetilde{\Psi}^{2}+a+\frac{\mathbf{k}^{2}}{4 m}\right) \operatorname{Re}^{2} \psi_{\mathbf{k}}+\left(b \widetilde{\Psi}^{2}+a+\frac{\mathbf{k}^{2}}{4 m}\right) \operatorname{Im}^{2} \psi_{\mathbf{k}}\right]\right\} .
\end{aligned}
$$

Carrying out the integral over the real and imaginary parts of the order parameter one can find an expression for the fluctuation part of the free energy:

$$
F=-\frac{T}{2} \sum_{\mathbf{k}}\left\{\ln \frac{\pi T_{c}}{3 b \widetilde{\Psi}^{2}+a+\frac{\mathbf{k}^{2}}{4 m}}+\ln \frac{\pi T_{c}}{b \widetilde{\Psi}^{2}+a+\frac{\mathbf{k}^{2}}{4 m}}\right\} .
$$

Let us discuss this result. It is valid both above and below $T_{c}$. The two terms in it correspond to the contributions of the modulus and phase fluctuations of the order parameter. Above $T_{c} \widetilde{\Psi} \equiv 0$ and these contributions are equal: phase and modulus fluctuations in the absence of $\widetilde{\Psi}$ represent just two equivalent degrees of freedom of the scalar complex order parameter. Below $T_{c}$, the symmetry of the system decreases (see (19)). The order parameter modulus fluctuations remain of the same diffusive type as above $T_{c}$, while the character of the phase fluctuations, in accordance with the Goldstone theorem, changes dramatically.

Substitution of (19) to (35) results in the disappearance of the temperature dependence of the phase fluctuation contribution and, calculating the second derivative, one sees that only the fluctuations of the order parameter modulus contribute to the heat capacity. As a result the heat capacity, calculated below $T_{c}$, turns out to be proportional to that found above:

$$
\delta C_{-}=2^{\frac{D}{2}-2} \delta C_{+} .
$$

Hence, in the framework of the theory proposed we found that the heat capacity of the superconductor tends to infinity at the transition temperature. Strictly speaking, the restrictions of the above approach do not permit us to 
discuss seriously this divergence at the critical point itself. The calculations in principle are valid only in that region of temperatures where the fluctuation correction is small. We will discuss in the next Section the quantitative criteria for the applicability of this perturbation theory.

\subsection{Ginzburg-Levanyuk criterion}

The fluctuation corrections to the heat capacity obtained above allow us to answer quantitatively the question: where are the limits of applicability of the GL theory?

This theory is valid not too near to the transition temperature, where the fluctuation correction is still small in comparison with the heat capacity jump. Let us define as the Ginzburg-Levanyuk number $G i_{(D)}$, 14,3 the value of the reduced temperature at which the fluctuation correction (30) equals the value of $\Delta C(21)$;

$$
G i_{(D)}=\frac{1}{\alpha}\left[\frac{V_{D}}{V} \vartheta_{D} b(4 m)^{\frac{D}{2}} T_{c}^{\frac{D}{2}-1}\right]^{\frac{2}{4-D}} .
$$

Substituting into this formula the microscopic values of the GL theory parameters (17) one can find

$$
G i_{(D)}=\left[\frac{7 \zeta(3) \vartheta_{D}}{8 \pi^{2}}\left(\frac{V_{D}}{V}\right) \frac{1}{\nu_{D} T_{c} \xi^{D}}\right]^{\frac{2}{4-D}} .
$$

Since $\nu_{D} T_{c} \sim \nu_{D} v_{F} / \xi_{c} \sim p_{F}^{D-1} \xi_{c}^{-1} \sim \mathfrak{a}^{1-D} \xi_{c}^{-1}$ one can convert this formula to the form

$$
G i_{(D)} \sim\left[\frac{7 \zeta(3) \vartheta_{D}}{8 \pi^{2}}\left(\frac{V_{D}}{V}\right) \frac{\xi_{c} \mathfrak{a}^{D-1}}{\xi^{D}}\right]^{\frac{2}{4-D}}
$$

where $\mathfrak{a}$ is the interatomic distance. It is worth mentioning that in bulk conventional superconductors, due to the large value of the coherence length $\left(\xi_{c} \sim 10^{-6} \div 10^{-4} \mathrm{~cm}\right)$, which drastically exceeds the interatomic distance $\left(\mathfrak{a} \sim 10^{-8} \mathrm{~cm}\right)$, the fluctuation correction to the heat capacity is extremely small. However, the fluctuation effect increases for small effective sample dimensionality and small electron mean free path. For instance, the fluctuation heat capacity of a superconducting granular system is readily accessible for experimental study.

\footnotetext{
${ }^{8}$ One can see that some arbitrariness occurs in this definition. For instance the $G i$ number could be defined as the reduced temperature at which the AL correction to conductivity is equal to the normal value of conductivity (as it was done in 13,14$)$. Such definition results in the change of the numerical factor in $G i$ number:

$$
G i_{(2, \sigma)}=1.44 G i_{(2, h . c .)} \text {. }
$$
}


Using the microscopic expression for the coherence length (1), the GinzburgLevanyuk number (37) can be evaluated for different cases of clean (c) and dirty (d) superconductors of various dimensionalities and geometries (film, wire, whisker and granule are supposed to have $3 D$ electronic spectrum):

\begin{tabular}{|c|c|c|c|}
\hline$G i_{(3)}$ & $G i_{(2)}$ & $G i_{(1)}$ & $G i_{(0)}$ \\
\hline $\begin{array}{l}80\left(\frac{T_{c}}{E_{F}}\right)^{4},(c) \\
\frac{1.6}{\left(p_{F} l\right)^{3}}\left(\frac{T_{c}}{E_{F}}\right),(d)\end{array}$ & $\begin{array}{l}\left(\frac{T_{c}}{E_{F}}\right),(c) \\
\frac{0.27}{p_{F} l},(d) \\
\frac{1.3}{p_{F}^{2} l d},(d) \text { film }\end{array}$ & $\begin{array}{l}0.5,(c) \\
1.3\left(p_{F}^{2} S\right)^{-2 / 3}\left(T_{c} \tau\right)^{-1 / 3},(d) \text { wire } \\
2.3\left(p_{F}^{2} S\right)^{-2 / 3},(c) \text { whisker }\end{array}$ & $\begin{array}{l}\frac{\sqrt{7 \zeta(3)}}{2 \pi} \frac{1}{\sqrt{\nu T_{c} V}} \approx \\
13.3 \frac{T_{c 0}}{E_{F}} \sqrt{\frac{\xi_{0}^{3}}{V}}\end{array}$ \\
\hline
\end{tabular}

Table 1.

One can see that for the three dimensional clean case the result coincides with the original Ginzburg evaluation and demonstrates the negligibility of the superconducting fluctuation effects in clean bulk materials.

\subsection{Scaling and renormalization group}

In the above study of the fluctuation contribution to heat capacity we have restricted ourselves to the temperature range out of the direct vicinity of the critical temperature: $|\epsilon| \gtrsim G i_{(D)}$. As we have seen the fluctuations in this region turn out to be weak and neglecting their interaction was justified. In this Section we will discuss the fluctuations in the immediate vicinity of the critical temperature $\left(|\epsilon| \lesssim G i_{(D)}\right)$ where this interaction turns out to be of great importance.

We will start with the scaling hypothesis, i.e. with the belief that in the immediate vicinity of the transition the only relevant length scale is $\xi(T)$. The temperature dependencies of all other physical quantities can be expressed through $\xi(T)$. This means, for instance, that the formula for the fluctuation part of the free energy (25) with the logarithm omitted is still valid in the region of critical fluctuations 9

$$
F_{(D)} \sim-\xi^{-D}(\epsilon),
$$

the coherence length is a power function of the reduced temperature: $\xi(\epsilon) \sim$ $\epsilon^{-\nu}$. The corresponding formula for the fluctuation heat capacity can be rewritten as

$$
\delta C \sim-\frac{\partial^{2} F}{\partial \epsilon^{2}} \sim \epsilon^{D \nu-2} .
$$

As was demonstrated in the Introduction, the GL functional approach, where the temperature dependence of $\xi(T)$ is determined only by the diffusion of the electrons forming Cooper pair, $\xi(\epsilon) \sim \epsilon^{-1 / 2}$ and $\delta C \sim \epsilon^{-1 / 2}$.

\footnotetext{
${ }^{9}$ The logarithm in (25) is essential for the case $D=2$. This case will be discussed later.
} 


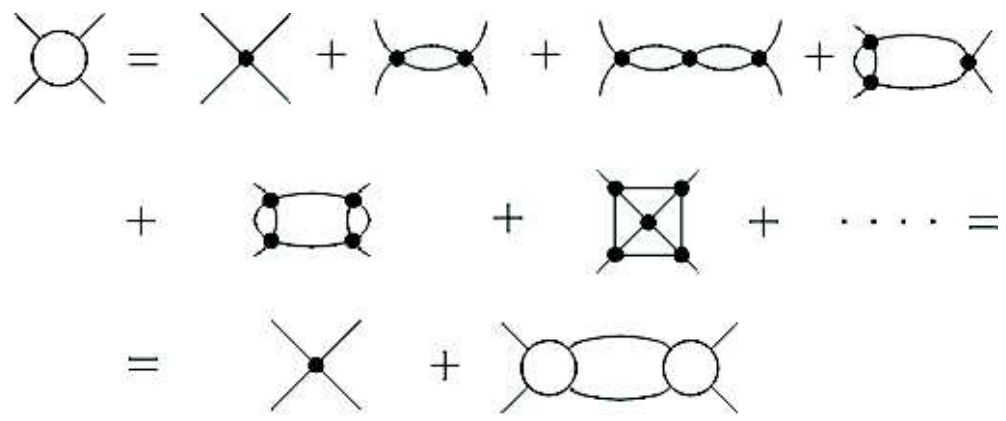

Fig. 2. Examples of diagrams for the fluctuation contribution to $b$.

These results are valid for the GL region $(|\epsilon| \gtrsim G i)$ only, where the interaction between fluctuations can be neglected. In the immediate vicinity of the transition (so-called critical region), where $|\epsilon| \lesssim G i$, the interaction of fluctuations becomes essential. Here fluctuation Cooper pairs affect the coherence length themselves, changing the temperature dependencies of $\xi(\epsilon)$ and $\delta C(\epsilon)$. In order to find the heat capacity temperature dependence in the critical region one would have to calculate the functional integral with the fourth order term, accounting for the fluctuation interaction, as was done for $0 D$ case. For the $3 D$ case up to now it is only known how to calculate a Gaussian type functional integral. This was done above when, for the GL region, we omitted the fourth order term in the free energy functional (16).

The first evident step in order to include in consideration of the critical region would be to develop a perturbation series in $b$ 10. Any term in this series has the form of a Gaussian integral and can be represented by a diagram, where the solid lines correspond to the correlators $\left\langle\Psi(r) \Psi^{*}\left(r^{\prime}\right)\right\rangle$. The "interactions " $b$ are represented by the points where four correlator lines intersect (see Fig. 2).

This series can be written as

$$
C \sim \sum_{n=0} c_{n}\left(\frac{G i_{(D)}}{\epsilon}\right)^{\frac{4-D}{2} n} .
$$

For $\epsilon \gtrsim G i$ it is enough to keep only the first two terms to reproduce the perturbational result obtained above. For $\epsilon \lesssim G i$ all terms have to be summed. It turns out that the coefficients $c_{n}$ can be calculated for the space dimensionality $D \rightarrow 4$ only. In this case the complex diagrams from Fig. 2 (like the diagram similar to an envelope) are small by the parameter $\varepsilon=4-D$ and

${ }^{10}$ Let us mention that this series is an asymptotic one, i.e. it does not converge even for small $b$. One can easily see this for small negative $b$, when the integral for the partition function evidently diverges. This is also confirmed by the exact $0 D$ solution (22). 
in order to calculate $c_{n}$ it is sufficient to sum the relatively simple "parquet" type diagrammatic series. Such a summation results in the substitution of the "bare" vertex $b$ by some effective interaction $\widetilde{b}$ which diminishes and tends to zero when the temperature approaches the transition point. Such a method was originally worked out in quantum field theory [15, 16, 17]. For the problem of a phase transition such a summation was first accomplished in [18.

Instead of a direct summation of the diagrams it is more convenient and physically obvious to use the method of the renormalization group. In the case of quantum field theory this method was known long ago 19,20. For phase transition theory it was proposed in [18 21] but the most simple and evident formulation was presented by Wilson 22. The idea of the renormalization group method consists in separating the functional integration over "fast" $\left(\psi_{|k|>\Lambda}\right)$ and "slow" $\left(\psi_{|k|<\Lambda}\right)$ fluctuation modes. If the cut off $\Lambda$ is large enough the fast mode contribution is small and the integration over them is Gaussian. After the first integration over fast modes the functional obtained depends on the slow ones only. They can, in their turn, be divided on slow $\left(|k|<\Lambda_{1}\right)$ and fast $\left(\Lambda_{1}<|k|<\Lambda\right)$, and the procedure can be repeated. Moving step by step ahead in this way one can calculate the complete partition function.

As an example of the first step of renormalization, the partition function calculation below $T_{c}$ can be recalled. There we separated the order parameter into the space-independent part $\widetilde{\Psi}$ ("slow" mode) and the fluctuation part $\psi(r)$ ("fast" mode) which was believed to be small in magnitude. Being in the GL region it was enough to average over the fast variables just once, while in the critical region the renormalization procedure requires subsequent approximations.

The cornerstone of the method consists in the fact that in the critical region at any subsequent step the free energy functional has the same form. For $D$ close to 4 this form coincides with the initial free energy GL functional but with the coefficients $a_{\Lambda}$ and $b_{\Lambda}$ depending on $\Lambda$. We will perform these calculations by the method of mathematical induction. Let us suppose that after the $(n-1)$-st step the free energy functional has the form:

$$
\begin{aligned}
\mathcal{F}\left[\widetilde{\Psi}_{\Lambda_{n-1}}\right]= & F_{N, \Lambda_{n-1}}+\int d V\left\{a_{\Lambda_{n-1}}\left|\widetilde{\Psi}_{\Lambda_{n-1}}\right|^{2}+\right. \\
& \left.+\frac{b_{\Lambda_{n-1}}}{2}\left|\widetilde{\Psi}_{\Lambda_{n-1}}\right|^{4}+\frac{1}{4 m}\left|\nabla \widetilde{\Psi}_{\Lambda_{n-1}}\right|^{2}\right\} .
\end{aligned}
$$

Writing $\widetilde{\Psi}_{\Lambda_{n-1}}$ in the form $\widetilde{\Psi}_{\Lambda_{n-1}}=\widetilde{\Psi}_{\Lambda_{n}}+\psi_{\Lambda_{n}}$ and choosing $\Lambda_{n}$ close enough to $\Lambda_{n-1}$ it is possible to make $\psi_{\Lambda_{n}}$ so small, that one can restrict the functional to the quadratic terms in $\psi_{\Lambda_{n}}$ only and perform the Gaussian integration in complete analogy with (34). The important property of the spaces with dimensionalities close to 4 is the possibility to choose $\Lambda_{n} \ll \Lambda_{n-1}$ and still to have $\psi_{\Lambda_{n}} \ll \widetilde{\Psi}_{\Lambda_{n}}$. In this case $\widetilde{\Psi}_{\Lambda_{n}}$ can be taken as coordinate independent, and one can use the result directly following from (34): 


$$
\begin{aligned}
\mathcal{F}\left[\widetilde{\Psi}_{\Lambda_{n}}\right]= & F_{N, \Lambda_{n-1}}+\int d V\left\{a_{\Lambda_{n}}\left|\widetilde{\Psi}_{\Lambda_{n}}\right|^{2}+\frac{b_{\Lambda_{n}}}{2}\left|\widetilde{\Psi}_{\Lambda_{n}}\right|^{4}+\frac{1}{4 m}\left|\nabla \widetilde{\Psi}_{\Lambda_{n}}\right|^{2}\right\} \\
& -\frac{T}{2} \sum_{\Lambda_{n}<|\mathbf{k}|<\Lambda_{n-1}}\left\{\ln \frac{\pi T_{c}}{\left(3 b_{\Lambda_{n}}\left|\widetilde{\Psi}_{\Lambda_{n}}\right|^{2}+a_{\Lambda_{n}}+\frac{\mathbf{k}^{2}}{4 m}\right)}+\right. \\
& \left.\ln \frac{\pi T_{c}}{b_{\Lambda_{n}}\left|\widetilde{\Psi}_{\Lambda_{n}}\right|^{2}+a_{\Lambda_{n}}+\frac{\mathbf{k}^{2}}{4 m}}\right\} .
\end{aligned}
$$

Expanding the last term in (41) in a series in $\widetilde{\Psi}_{\Lambda_{n}}$ one can get for $\mathcal{F}\left[\widetilde{\Psi}_{\Lambda_{n}}\right]$ the same expression (40) with the substitution of $\Lambda_{n-1} \rightarrow \Lambda_{n}$. From (41) follows that

$$
\begin{aligned}
& F_{n, \Lambda_{n}}=F_{n, \Lambda_{n-1}}-T \sum_{\Lambda_{n}<|\mathbf{k}|<\Lambda_{n-1}} \ln \frac{\pi T_{c}}{\left(a_{\Lambda_{n}}+\frac{\mathbf{k}^{2}}{4 m}\right)}, \\
& a_{\Lambda_{n}}=a_{\Lambda_{n-1}}+2 T \sum_{\Lambda_{n}<|\mathbf{k}|<\Lambda_{n-1}} \frac{b_{\Lambda_{n}}}{\left(a_{\Lambda_{n}}+\frac{\mathbf{k}^{2}}{4 m}\right)} \\
& b_{\Lambda_{n}}=b_{\Lambda_{n-1}}-5 T \sum_{\Lambda_{n}<|\mathbf{k}|<\boldsymbol{\Lambda}_{n-1}} \frac{b_{\Lambda_{n}}^{2}}{\left(a_{\Lambda_{n}}+\frac{\mathbf{k}^{2}}{4 m}\right)^{2}} .
\end{aligned}
$$

Passing to a continuous variable $\Lambda_{n} \rightarrow \Lambda$ one can rewrite these recursion equations as the set of differential equations:

$$
\begin{aligned}
& \frac{\partial F(\Lambda)}{\partial \Lambda}=-T \mu_{D} \Lambda^{D-1} \ln \frac{\pi T_{c}}{\left(a(\Lambda)+\frac{\Lambda^{2}}{4 m}\right)}, \\
& \left\{\begin{array}{l}
\frac{\partial a(\Lambda)}{\partial \Lambda}=-2 T \mu_{D} \frac{b(\Lambda) \Lambda^{D-1}}{\left(a(\Lambda)+\frac{\Lambda^{2}}{4 m}\right)} \\
\frac{\partial b(\Lambda)}{\partial \Lambda}=5 T \mu_{D} \frac{b^{2}(\Lambda) \Lambda^{D-1}}{\left(a(\Lambda)+\frac{\Lambda^{2}}{4 m}\right)^{2}}
\end{array}\right.
\end{aligned}
$$

These renormalization group equations are evidently valid for small enough $\Lambda$ only, where the transition from discrete to continuous variables is justified. This means at least

$$
\Lambda^{2} / 4 m \ll T_{c 0} G i_{(D)} .
$$

in order to move away from the first approximation.

Let us recall that in the framework of the Landau theory of phase transitions the coefficient $a\left(T_{c 0}\right)=0$ at the transition point and this can be considered as the MFA definition of the critical temperature $T_{c 0}$. The same statement for the function $a=a(\Lambda)$ in the framework of the renormalization 
group method can be written as the $a\left(T_{c 0}, \Lambda \sim \xi^{-1}\right)=0$. With the decrease of $\Lambda$ the effect of critical fluctuations more and more is taken into account and the renormalized value of the critical temperature decreases, being defined by the equation: $a\left(T_{c}(\Lambda), \Lambda\right)=0$. Finally, after the application of the complete renormalization procedure, one can define the real critical temperature $T_{c}$, shifted down with respect to $T_{c 0}$ due to the effect of fluctuations, from the equation:

$$
a\left(T_{c}, \Lambda=0\right)=0 .
$$

It is easy to find this shift in the first approximation. Indeed, let us integrate the first equation of (44) over $\Lambda$ in limits $\left[0, \xi^{-1}\right]$. The main contribution to the integral will be determined by the region where $a(\Lambda) \ll \frac{\Lambda^{2}}{4 m}$. Being far from the critical point one can assume that the coefficient $b=$ const and then

$$
a\left(\xi^{-1}\right)=\alpha \delta T_{c}=\int_{a(0)}^{a\left(\xi^{-1}\right)} d a=-8 m T \mu_{D} b \int_{0}^{1 / \xi} \Lambda^{D-3} d \Lambda .
$$

For the $3 D$ case this gives the shift of the critical temperature $\delta T_{c}$ due to fluctuations 11

$$
\frac{\delta T_{c}^{(3)}}{T_{c}} \sim-\frac{2 m b}{\pi \alpha \xi}=-\frac{b}{2 \pi T_{c} \alpha^{2}} \frac{1}{\xi^{3}}=-\frac{7 \zeta(3)}{16 \pi^{3} \nu T_{c} \xi^{3}}=-\frac{8}{\pi} \sqrt{G i_{(3)}} .
$$

Let us come back to study of properties of the system of equations (44). One can find its partial solution at $T=T_{c}$ in the form:

$$
\begin{aligned}
a\left(T_{c}, \Lambda\right) & =\frac{4-D}{4 m[5+(4-D)]} \Lambda^{2} \\
b\left(T_{c}, \Lambda\right) & =\frac{5}{16 m^{2} T \mu_{D}} \frac{4-D}{[5+(4-D)]^{2}} \Lambda^{4-D} .
\end{aligned}
$$

These power solutions are correct in the domain of validity of the system (44) itself, i.e. for small enough $\Lambda$ defined by the condition (45). Nevertheless, in a space of dimensionality close to $4(D=4-\varepsilon, \varepsilon \ll 1)$ it is possible to extend their validity up to the GL region and to observe their crossover to

${ }^{11}$ In the same way we can analyze the shift of the critical temperature in $2 D$ case too and obtain:

$$
\frac{\delta T_{c}^{(2)}}{T_{c}}=-2 G i_{(2)} \ln \frac{1}{4 G i_{(2)}} .
$$

As we will show below both $3 D$ and $2 D$ results for $\delta T_{c}$ coinside with those obtained by the analysis of the effect of fluctuations on superconducting density in the perturbation approach. 
the GL results: $a\left(T_{c}\right)=0 ; b\left(T_{c}\right)=b_{0}=$ const. Indeed, in this case, due to proportionality of $a(\Lambda)$ to $\varepsilon \rightarrow 0$, one can omit it in the denominators of the system (44) and to write down the solution for $b(\Lambda)$ in the form

$$
b^{-1}\left(T_{c}, \Lambda\right)=b_{0}^{-1}+\frac{80 m^{2}}{(4-D)} T \mu_{D}\left(\Lambda^{D-4}-\xi^{4-D}\right) .
$$

We have chosen the constant of integration as $b_{0}^{-1}-\frac{80 m^{2}}{(4-D)} T \mu_{D} \xi^{4-D}$ in order to match the renormalization group and GL solutions at the value of $\Lambda=$ $\Lambda_{\max } \sim \xi^{-1}$.

Now let us pass to study of the function $a(T, \Lambda)$ for the same interesting case of space dimensionality $D \rightarrow 4$ for temperatures slightly different ( but still close enough) from $T_{c}$, where one can write

$$
a(T, \Lambda)=a\left(T_{c}, \Lambda\right)+\alpha\left(T_{c}, \Lambda\right) T_{c} \epsilon .
$$

The first term on the right hand side is determined by Eq. (49). In order to determine $\alpha\left(T_{c}, \Lambda\right)$ let us expand the first equation in (44) in terms of $\epsilon$

$$
\frac{\partial \alpha\left(T_{c}, \Lambda\right)}{\partial \Lambda}=2 T \mu_{D} \frac{b\left(T_{c}, \Lambda\right) \Lambda^{D-1}}{\left(a\left(T_{c}, \Lambda\right)+\frac{\Lambda^{2}}{4 m}\right)^{2}} \alpha\left(T_{c}, \Lambda\right) .
$$

For

$$
\Lambda^{2} / 4 m \gtrsim \alpha\left(T_{c}, \Lambda\right) T_{c} \epsilon
$$

we can again use the solution (50) for $b\left(T_{c}, \Lambda\right)$ and omit $a\left(T_{c}, \Lambda\right)$ in the denominator of (51). The constant of integration, appearing in the process of solution of (51), is chosen in accordance with the condition that for $\Lambda=$ $\Lambda_{\max } \sim \xi^{-1}$ we match $\alpha\left(T_{c}, \Lambda\right)=\alpha\left(T_{c 0}, \xi^{-1}\right)=\alpha_{0}$ with the GL theory:

$$
\alpha\left(T_{c}, \Lambda\right)=\alpha_{0}\left[1+\frac{80 m^{2} b_{0}}{(4-D)} T \mu_{D}\left(\Lambda^{D-4}-\xi^{4-D}\right)\right]^{-2 / 5} .
$$

The condition (22) can be written as $\Lambda \gtrsim \xi^{-1}(T)$, where $\xi(T)$ is the generalized coherence length, determined by the equation:

$$
\xi^{-2}(T)=4 m \alpha\left(T_{c}, \xi^{-1}(T)\right) T_{c} \epsilon .
$$

Such a definition is valid at any temperature. For example, far enough from the critical point, in the GL region, $\alpha\left(T_{c}, \xi^{-1}(T)\right)=\alpha_{0}$ and one reproduces the result (3). Vice versa, in the critical region the main contribution on the right hand side of the Eq. (53) results from the second term containing $\Lambda^{D-4}$ so, putting $\xi^{-1}(T)=\Lambda$, one can rewrite the self-consistent equation for $\xi(T)$ and get

$$
\xi(T)=(4 m)^{(1-D) / 2} \frac{4-D}{20 b_{0} T \mu_{D} \sqrt{T_{c} \alpha_{0}}} \epsilon^{-\nu},
$$


where $2 \nu=[1-(4-D) / 5]^{-1}$. As was already mentioned, strictly speaking this result was carried out for $\varepsilon=4-D \ll 1$, so it is confident up to the first in $\varepsilon$ expansion only: $\nu=1 / 2+\varepsilon / 10$. Nevertheless extending it to $\varepsilon=1$ $(D=3)$ one can obtain $\nu_{3}=3 / 5$.

Let us pass to the calculation of the critical exponent of the heat capacity in the immediate vicinity of the transition. For this purpose one can calculate the second derivative of equation (43) with respect to $\epsilon$ :

$$
\frac{\partial C(\Lambda)}{\partial \Lambda}=T^{2} \mu_{D} \frac{\Lambda^{D-1} \alpha^{2}(\Lambda)}{\left(\alpha(\Lambda) T_{c} \epsilon+\frac{\Lambda^{2}}{4 m}\right)^{2}}
$$

The heat capacity renormalized by fluctuations has the value $C(\Lambda=0)$ which is the result of integration over all fluctuation degrees of freedom. Carrying out the integration of (55) over all $\Lambda \lesssim \xi^{-1}$ one can divide the domain of integration on the right hand side in two: $\Lambda \lesssim \xi^{-1}(T)$ and $\xi^{-1}(T) \lesssim \Lambda \lesssim$ $\xi^{-1}$. In the calculation of the integral over the region $\xi>\Lambda \gtrsim \xi^{-1}(T)$ the inequality $\alpha(\Lambda) T_{c} \epsilon \ll \frac{\Lambda^{2}}{4 m}$ holds, and the function $\alpha(\Lambda)$ can be omitted in the denominator. In the numerator of (55) one can use for $\alpha(\Lambda)$ the solution (53). In the region $\Lambda \lesssim \xi^{-1}(T)$ one has to use the partial solution (49) for $\alpha(\Lambda)$ and can find that the contribution of this domain has the same singularity as that from the region $\Lambda \gtrsim \xi^{-1}(T)$, but with a coefficient proportional to $(4-D)^{2}=\varepsilon^{2}$, hence negligible in our approximation. The result is:

$$
C(\Lambda=0)=\alpha_{0}^{2}\left[(4 m T)^{2} \mu_{D}\right]^{\frac{1}{5}}\left[\frac{4}{5} \frac{(4-D)}{b_{0}}\right]^{4 / 5} \frac{5}{4-D} \xi^{\frac{4-D}{5}}(T) .
$$

Substituting the expression for $\xi(T)$ one can finally find

$$
C=2^{12 / 5} \alpha_{0}^{2}\left[5 \mu_{D} \frac{m^{2} T^{2}}{b_{0}^{4}(4-D)}\right]^{\frac{1}{5}} \epsilon^{-\alpha},
$$

confirming the validity of the scaling hypothesis and the relation (39). The critical exponent in (57) is

$$
\alpha=\frac{(4-D)}{10[1-(4-D) / 5]} \approx \varepsilon / 10 .
$$

One can see that generally speaking the critical exponents $\nu$ and $\alpha$ appear in the form of series in powers of $\varepsilon$. More cumbersome calculations permit finding the next approximations for them in $\varepsilon=4-D$. Nevertheless it is worth mentioning that even the first approximation, giving $\nu_{3}=3 / 5$ and $\alpha_{3}=1 / 10$ for $\varepsilon=1$, is already weakly affected by the following steps of the expansion in powers of $\varepsilon$ [23].

One can notice that the exercise performed in this Section has more academic than practical character. Indeed, the results obtained turn out applicable to the analysis of the critical region of a $3 D$ superconductor only if 
$G i$ is so small that the theoretical predictions are hardly experimentally observable. Nevertheless we demonstrated the RG method which helps to see the complete picture of the fluctuations manifestation in the vicinity of the critical temperature.

\subsection{Fluctuation diamagnetism}

Qualitative preliminaries. In this Section we discuss the effect of fluctuations on the magnetization and the susceptibility of a superconductor above the transition temperature. Being the precursor effect for the Meissner diamagnetism, the fluctuation induced magnetic susceptibility has to be a small correction with respect to the diamagnetism of a superconductor but it can be comparable to or even exceed the value of the normal metal diamagnetic or paramagnetic susceptibility and can be easily measured experimentally. As was already mentioned in the Introduction the temperature dependence of the fluctuation induced diamagnetic susceptibility can be qualitatively analyzed on the basis of the Langevin formula, but some precautions in the case of low dimensional samples have to be made.

As regards the $3 D$ case we would like just to mention here that Exp.(12), presented in terms of $\xi(T)$, has a wider region of applicability than the GL one. Namely, the scaling arguments are valid for diamagnetic susceptibility too and one can write the general relation

$$
\chi_{(3)} \sim-e^{2} T \xi(T) \sim-\chi_{P} \epsilon^{-1 / 2}\left\{\begin{array}{l}
1, \epsilon \gtrsim G i \\
\left(\frac{\epsilon}{G i}\right)^{1 / 2-\nu}, \epsilon \lesssim G i
\end{array},\right.
$$

which is valid in the region of critical fluctuations in the immediate vicinity of the transition temperature too. Here, in order to define the scale of fluctuation effects, we have introduced the Pauli paramagnetic susceptibility $\chi_{P}=e^{2} v_{F} / 4 \pi^{2}$. Moreover, the Langevin formula permits us to extend the estimation of the fluctuation diamagnetic effect to the other side beyond the GL region: to high temperatures $T \gg T_{c}$. The coherence length far from the transition becomes a slow function of temperature. In a clean superconductor, far from $T_{c}, \xi(T) \sim v_{F} / T$, so one can write

$$
\chi_{(3 c)}\left(T \gg T_{c}\right) \sim-e^{2} T \xi(T) \sim-\chi_{P}
$$

and see that the fluctuation diamagnetism turns out to be of the order of the Pauli paramagnetism even far from the transition. More precise microscopic calculations of $\chi_{(3)}\left(T \gg T_{c}\right)$ lead to the appearance of $\ln ^{2}\left(T / T_{c}\right)$ in the denominator of (59).

In the $2 D$ case Exp.(12) is applicable for the estimation of $\chi_{(2)}$ in the case when the magnetic field is applied perpendicular to the plane, permitting $2 D$ rotations of fluctuation Cooper pairs in it:

$$
\chi_{(2 c)}(T) \sim e^{2} \frac{n}{m}<R^{2}>\sim e^{2} T \xi^{2}(T) \sim-\chi_{P} \frac{E_{F}}{T-T_{c}} .
$$


This result is valid for a wide range of temperatures and can exceed the Pauli paramagnetism by factor $\frac{E_{F}}{T}$ even far from the critical point (we consider the clean case here).

For a thin film $(d \ll \xi(T))$ perpendicular to the magnetic field the fluctuation Cooper pairs behave like effective $2 D$ rotators, and the formula (12) still can be used, though one has to take into account that the susceptibility in this case is calculated per unit square of the film. So for the realistic case (from the experimental point of view) of the dirty film, one has just use in (60) the expression (6) for the coherence length:

$$
\chi_{(2 d)} \sim \frac{e^{2} T}{d} \xi^{2}(T) \sim-\chi_{P}\left(\frac{l}{d}\right) \frac{T_{c}}{T-T_{c}} .
$$

Let us discuss now the important case of a layered superconductor (for example, a high temperature superconductor). It is usually supposed that the electrons move freely in conducting planes separated by a distance $s$. Their motion in the perpendicular direction has a tunneling character, with effective energy $J$. The related velocity and coherence length can be estimated as $v_{z}=\partial E(\mathbf{p}) / \partial p_{\perp} \sim J / p_{\perp} \sim s J$ and $\xi_{z,(c)} \sim s J / T$ for clean case. In dirty case the anisotropy can be taken into account in the spirit of formula (6) yielding $\xi_{z,(d)} \sim \sqrt{D_{\perp} / T} \sim s J \sqrt{\tau / T}$.

We start from the case of a weak magnetic field applied perpendicular to layers. The effective area of a rotating fluctuation pair is $\xi_{x}(\epsilon) \xi_{y}(\epsilon)$. The density of Cooper pairs in the conducting layers (10) has to be modified for the anisotropic case. Its isotropic $3 D$ value is proportional to $1 / \xi(\epsilon)$, that now has to be read as $\sim 1 / \sqrt{\xi_{x}(\epsilon) \xi_{y}(\epsilon)}$. The anisotropy of the electron motion leads to a concentration of fluctuation Cooper pairs in the conducting layers and hence, to an effective increase of the Cooper pairs density of $\sqrt{\xi_{x}(\epsilon) \xi_{y}(\epsilon)} / \xi_{z}(\epsilon)$ times its isotropic value. This increase is saturated when $\xi_{z}(\epsilon)$ reaches the interlayer distance $s$, so finally the anisotropy factor appears in the form $\sqrt{\xi_{x}(\epsilon) \xi_{y}(\epsilon)} / \max \left\{s, \xi_{z}(\epsilon)\right\}$ and the square root in its numerator is removed in the Langevin formula (12), rewritten for this case

$$
\chi_{(\text {layer }, \perp)}(\epsilon, H \rightarrow 0) \sim-e^{2} T \frac{\xi_{x}(\epsilon) \xi_{y}(\epsilon)}{\max \left\{s, \xi_{z}(\epsilon)\right\}} .
$$

The existence of a crossover between the $2 D$ and $3 D$ temperature regimes in this formula is evident: as the temperature tends to $T_{c}$ the diamagnetic susceptibility temperature dependence changes from $1 / \epsilon$ to $1 / \sqrt{\epsilon}$. This happens when the reduced temperature reaches its crossover value $\epsilon_{c r}=r$ $\left(\xi_{z}\left(\epsilon_{c r}\right) \sim s\right)$. The anisotropy parameter

$$
r=\frac{4 \xi_{z}^{2}(0)}{s^{2}}=\frac{J^{2}}{T}\left\{\begin{array}{l}
\frac{\pi \tau}{4}, T \tau \ll 1 \\
\frac{7 \zeta(3)}{8 \pi^{2} T}, T \tau \gg 1
\end{array}\right.
$$

plays an important role in the theory of layered superconductors 12 .

$\overline{12}$ We use here a definition of $r$ following from microscopic theory (see Section 6 ). 
It is interesting to note that this intrinsic crossover, related to the spectrum anisotropy, has an opposite character to the geometric crossover which happens in thick enough films when $\xi(T)$ reaches $d$. In the latter case the characteristic $3 D \quad 1 / \sqrt{\epsilon}$ - dependence taking place far enough from $T_{c}$ (where $\xi(T) \ll d)$, is changed to the $2 D 1 / \epsilon$ law (see (61)) in the immediate vicinity of transition ( where $\xi(T) \gg d$ ) [24]. It is worth mentioning that in a strongly anisotropic layered superconductor the fluctuation-induced susceptibility may considerably exceed the normal metal dia- and paramagnetic effects even relatively far from $T_{c}$ [25,26].

Let us consider a magnetic field applied along the layers. First it is necessary to mention that the fluctuation diamagnetic effect disappears in the limit $J \sim \xi_{z} \rightarrow 0$. Indeed, for the formation of a circulating current it is necessary to tunnel twice, so

$$
\chi_{(\text {layer }, \|)} \sim-e^{2} T \frac{\xi_{z}^{2}}{\max \left\{s, \xi_{z}(T)\right\}} \sim-\chi_{P}\left(\frac{s J}{v_{F}}\right) \frac{J / T}{\sqrt{\epsilon} \max \{\sqrt{\epsilon}, J / T\}} .
$$

In the general case of an anisotropic superconductor, choosing the $z$ axis along the direction of magnetic field $H$, the following extrapolation of the results obtained may be written

$$
\chi \sim-e^{2} T \frac{\xi_{x}^{2}(\epsilon) \xi_{y}^{2}(\epsilon)}{\max \left\{\mathfrak{a}, \xi_{x}(\epsilon)\right\} \max \left\{\mathfrak{b}, \xi_{y}(\epsilon)\right\} \max \left\{\mathfrak{s}, \xi_{z}(\epsilon)\right\}} .
$$

This general formula is useful for the analysis of the fluctuation diamagnetism of anisotropic superconductors or samples of some specific shape: granular, quasi- $1 D$, quasi- $2 D$, and $3 D$. It is also applicable to the case of a thin film $\left(d \ll \xi_{z}(\epsilon)\right)$ placed perpendicular to the magnetic field: it is enough to replace $\xi_{z}(\epsilon)$ by $d$ in (64). Nevertheless the formula (64) cannot be applied to the cases of thin films in parallel fields, wires and granules. In those cases the Langevin formula (12) can still be used with the replacement of $\left\langle R^{2}\right\rangle \rightarrow d^{2}$ :

$$
\chi_{(D)} \sim-\chi_{P}\left(\frac{T}{v_{F}}\right) \xi^{2-D} d^{D-1} \sim \epsilon^{D / 2-1} .
$$

The magnetic field dependence of the fluctuation part of free energy in these cases is reduced only to account for the quadratic shift of the critical temperature versus magnetic field.

For $3 D$ systems or in the case of a film in a perpendicular magnetic field the critical temperature depends on $H$ linearly, while the magnetic field dependent part of the free energy for $H \ll H_{c 2}^{*}(-\epsilon)$ (the line $H_{c 2}^{*}(-\epsilon)$ is mirrorsymmetric to the $H_{c 2}(\epsilon)$ with respect to $y$-axis passing through $\left.T=T_{c}\right)$ is proportional to $H^{2}$. This is why the magnetic susceptibility is determined by Eq. (64) for weak enough magnetic fields $H \ll \Phi_{0} /\left[\xi_{x}(\epsilon) \xi_{y}(\epsilon)\right]=H_{c 2}(\epsilon) \ll$ $H_{c 2}(0)$ only. In the vicinity of $T_{c}$ these fields are small enough. 
Zero-dimensional diamagnetic susceptibility. For quantitative analysis of the fluctuation diamagnetism we start by writing down the GL functional for the free energy (see Exp.(16)) in the presence of the magnetic field

$$
\begin{aligned}
\mathcal{F}[\Psi(\mathbf{r})]= & F_{n}+\int d V\left\{a|\Psi(\mathbf{r})|^{2}+\frac{b}{2}|\Psi(\mathbf{r})|^{4}+\frac{1}{4 m}|(-i \nabla-2 e \mathbf{A}) \Psi(\mathbf{r})|^{2}+\right. \\
& \left.+\frac{\mathbf{B}^{2}}{8 \pi}-\frac{\mathbf{H} \cdot \mathbf{B}}{4 \pi}\right\} .
\end{aligned}
$$

where $\mathbf{A}$ is vector potential. As long as fluctuation effects are comparatively small, the average magnetic field in the metal $\mathbf{B}$ may be assumed to be equal to the external field $\mathbf{H}$. Thus we omit the last two terms in (65) (see later on).

The fluctuation contribution to the diamagnetic susceptibility in the simplest case of a "zero-dimensional" superconductor (spherical superconducting granule of diameter $d \ll \xi(\epsilon))$ was considered by V.Shmidt [11]. In this case the order parameter does not depend on the space variables and the free energy can be calculated exactly for all temperatures including the critical region in the same way as was done for the case of the heat capacity in the absence of a magnetic field. Formally the effect of a magnetic field in this case is reduced to the renormalization of the coefficient $a$, or, in other words, to the suppression of the critical temperature. This is why one can use the same formula 222 for the partition function with the critical temperature $T_{c}$ shifted by magnetic field as: 13 .

$$
T_{c}(H)=T_{c}(0)\left(1-\frac{4 \pi^{2} \xi^{2}}{\Phi_{0}^{2}}<\mathbf{A}^{2}>\right) .
$$

Here $\Phi_{0}=\frac{\pi}{e}$ is the magnetic flux quantum and $<\ldots . .>$ means the averaging over the sample volume.

Such a trivial dependence of the properties of $0 D$ samples on magnetic field immediately allows one to understand its effect on the heat capacity of a granular sample. Indeed, with the growth of the field the temperature dependence of the heat capacity presented in Fig. 1 1 just moves in the direction of lower temperatures.

In the GL region $G i_{(0)} \lesssim \epsilon$ one can write the asymptotic expression (23) for the free energy:

$$
F_{(0)}(\epsilon, H)=-T \ln \frac{\pi}{\alpha\left(\epsilon+\frac{4 \pi^{2} \xi^{2}}{\Phi_{0}^{2}}<\mathbf{A}^{2}>\right)} .
$$

In the case of a spherical particle the relation $\left\langle\mathbf{A}^{2}\right\rangle=\frac{1}{10} H^{2} d^{2}$ can be used in full analogy with the calculation of the moment of inertia of a solid sphere.

\footnotetext{
${ }^{13}$ Let us stress the difference between the $H^{2}$ shift of the critical temperature for
} a zero-dimensional granule and the linear shift in the case of bulk material. 
In this way an expression for the $0 D$ fluctuation magnetization valid for all fields $H \ll H_{c 2}(0)$ can be found:

$$
M_{(0)}(\epsilon, H)=-\frac{\partial F_{(0)}(\epsilon, H)}{\partial H}=-T \frac{\frac{2 \pi^{2} \xi^{2}}{5 \Phi_{0}^{2}} d^{2}}{\left(\epsilon+\frac{\pi^{2} \xi^{2}}{5 \Phi_{0}^{2}} H^{2} d^{2}\right)} H .
$$

One can see that the fluctuation magnetization turns out to be negative and linear up to some crossover field, which can be called the temperature dependent upper critical field of the granule $H_{c 2(0)}(\epsilon) \sim \frac{\Phi_{0}}{d \xi(\epsilon)}=\frac{\xi}{d} H_{c 2}(0) \sqrt{\epsilon}$ at which it reaches a minimum. At higher fields $H_{c 2(0)}(\epsilon) \lesssim H \ll H_{c 2}(0)$ the fluctuation magnetization of the $0 D$ granule decreases as $1 / H$. In the weak field region $H \ll H_{c 2(0)}(\epsilon)$ the diamagnetic susceptibility is:

$$
\chi_{(0)}(\epsilon, H)=-\frac{12 \pi T \xi_{0}^{2}}{5 \Phi_{0}^{2} d} \frac{1}{\epsilon} \approx-2 \cdot 10^{2} \chi_{P}\left(\frac{\xi}{d}\right) \frac{1}{\epsilon}
$$

which coincides with our previous estimate in its temperature dependence but the numerical factor found is very large. Let us underline that the temperature dependence of the $0 D$ fluctuation diamagnetic susceptibility turns out to be less singular than the $0 D$ heat capacity correction: $\epsilon^{-1}$ instead of $\epsilon^{-2}$.

The expression for the fluctuation part of free energy (28) is also applicable to the cases of a wire or a film placed in a parallel field: as was already mentioned above all its dependence on magnetic field is manifested by the shift of the critical temperature (66). In the case of the wire in a parallel field one has to choose the gauge of the vector-potential $\mathbf{A}=\frac{1}{2} \mathbf{H} \times \mathbf{r}$ yielding $\left\langle\mathbf{A}^{2}\right\rangle_{(\text {wire }, \|)}=\frac{H^{2} d^{2}}{32}$ (the calculation of this average is analogous to that of the moment of inertia of a solid sphere). For a wire in a perpendicular field, or a film in a parallel field, the gauge has to be chosen in the form $\mathbf{A}=(0, H x, 0)$ (to avoid the appearance of currents perpendicular to surface). One can find $\left\langle\mathbf{A}^{2}\right\rangle_{(\text {wire }, \perp)}=\frac{H^{2} d^{2}}{16}$ for a wire and $\left\langle\mathbf{A}^{2}\right\rangle_{(\text {film. } .)}=\frac{H^{2} d^{2}}{12}$ for a film.

Calculating the second derivative of Eq.(28) with the appropriate magnetic field dependencies of the critical temperature one can find the following expressions for the diamagnetic susceptibility:

$$
\chi_{(D)}(\epsilon)=-2 \pi \frac{\xi T}{v_{F}} \chi_{P}\left\{\begin{array}{l}
\frac{1}{\sqrt{\epsilon}}, \text { wire in parallel field } \\
\frac{2}{\sqrt{\epsilon}}, \text { wire in perpendicular field } \\
\frac{d}{3 \xi} \ln \frac{1}{\epsilon}, \text { film in parallel field }
\end{array}\right.
$$

GL treatment of fluctuation magnetization. Let us analyze quantitatively, on the basis of the GL functional, the temperature and field dependencies of the fluctuation magnetization. We will carry on the discussion for a layered superconductor. As was already mentioned this system has a great 
practical importance because of its direct applicability to high temperature superconductors, where the fluctuation effects are very noticeable. Moreover, the general results obtained will allow us to analyze $3 D$ and $2 D$ situations as limiting cases. The effects of a magnetic field are more pronounced for perpendicular orientation, so let us consider first this case.

The generalization of the GL functional for a layered superconductor (Lawrence-Doniach (LD) functional [27) in a perpendicular magnetic field can be written as

$$
\begin{aligned}
\mathcal{F}_{L D}[\Psi]= & \sum_{l} \int d^{2} r\left(a\left|\Psi_{l}\right|^{2}+\frac{b}{2}\left|\Psi_{l}\right|^{4}+\left.\frac{1}{4 m}\left|\left(\nabla_{\|}-2 i e \mathbf{A}_{\|}\right)\right| \Psi_{l}\right|^{2}\right. \\
& \left.+\mathcal{J}\left|\Psi_{l+1}-\Psi_{l}\right|^{2}\right),
\end{aligned}
$$

where $\Psi_{l}$ is the order parameter of the $l$-th superconducting layer and the phenomenological constant $\mathcal{J}$ is proportional to the Josephson coupling between adjacent planes. The gauge with $A_{z}=0$ is chosen in (69). In the immediate vicinity of $T_{c}$ the LD functional is reduced to the GL one with the effective mass $M=\left(4 \mathcal{J} s^{2}\right)^{-1}$ along $c$-direction, where $s$ is the inter-layer spacing. One can relate the value of $\mathcal{J}$ to the coherence length along the c-direction: $\mathcal{J}=2 \alpha T_{c} \xi_{z}^{2} / s^{2}$. Since we are dealing with the GL region the fourth order term in $(69)$ can be omitted.

As it is well known the Landau representation is the most appropriate for problems related with the motion of a charged particle in a uniform magnetic field. The fluctuation Cooper pair wave function $\phi_{n k_{z}}(\mathbf{r})$ can be written as the product of a plane wave propagating along the magnetic field direction and a Landau state wave function. Let us expand the order parameter $\Psi_{l}(\mathbf{r})$ on the basis of these eigenfunctions:

$$
\Psi_{l}(\mathbf{r})=\sum_{\mathbf{n}, k_{z}} \Psi_{n, k_{z}} \phi_{n k_{z}}(\mathbf{r}) \exp \left(i k_{z} l\right)
$$

where $\mathbf{n}$ is the quantum number related with the degenerate Landau state and $k_{z}$ is the momentum component along the direction of the magnetic field. Substituting this expansion into (69) one can find the LD free energy as a functional of the $\Psi_{n, k_{z}}$ coefficients:

$$
\mathcal{F}_{L D}\left[\Psi_{n, k_{z}}\right]=\sum_{\mathbf{n}, k_{z}}\left\{\alpha T_{c} \epsilon+\frac{H}{2 m \Phi_{0}}\left(n+\frac{1}{2}\right)+\mathcal{J}\left(1-\cos \left(k_{z} s\right)\right)\right\}\left|\Psi_{n, k_{z}}\right|^{2} .
$$

In complete analogy with the case of an isotropic spectrum the functional integral over the order parameter configurations $\Psi_{n, k_{z}}$ in the partition function can be reduced to a product of ordinary Gaussian integrals, and the fluctuation part of the free energy in a magnetic field takes the form:

$$
F(\epsilon, H)=-\frac{2 \pi S H}{\Phi_{0}} T \sum_{n, k_{z}} \ln \frac{\pi T}{\alpha T_{c} \epsilon+\frac{H}{2 m \Phi_{0}}\left(n+\frac{1}{2}\right)+J\left(1-\cos \left(k_{z} s\right)\right)} .
$$


Here the summation over the degenerate states of each Landau level was performed ( $S$ is the sample cross-section) and results in appearance of the number of particle states $\left(2 \pi H S / \Phi_{0}\right)$ with the definite quantum numbers $n$ and $k_{z}$. The summation over $n$ has to be performed through all occupied states, i.e. the upper limit of the sum is $N \sim 2 m \Phi_{0} E_{F} / H$.

In the limit of weak fields one can carry out the summation over the Landau states by means of the Euler-Maclaurin's transformation

$$
\sum_{n=0}^{N} f(n)=\int_{-1 / 2}^{N+1 / 2} f(n)-\frac{1}{24}\left[f^{\prime}(N+1 / 2)-f^{\prime}(-1 / 2)\right]
$$

and obtain

$$
F(\epsilon, H)=F(\epsilon, 0)+\frac{\pi S T H^{2}}{24 m \Phi_{0}^{2}} \int_{-\pi / s}^{\pi / s} \frac{\mathcal{N} s d k_{z}}{2 \pi}\left\{\frac{1}{\alpha T_{c} \epsilon+\mathcal{J}\left(1-\cos \left(k_{z} s\right)\right)}\right\} .
$$

Here $\mathcal{N}$ is the total number of layers. After the momentum integration one gets:

$$
F(\epsilon, H)=F(\epsilon, 0)+\frac{\pi V H^{2}}{24 m \alpha s \Phi_{0}^{2}} \frac{1}{\sqrt{\epsilon(\epsilon+r)}}
$$

with the anisotropy parameter defined as 4

$$
r=\frac{2 \mathcal{J}}{\alpha T}=\frac{4 \xi_{z}^{2}(0)}{s^{2}} .
$$

The magnetic susceptibility in a weak field turns out 28,29] to be

$$
\chi_{(\text {layer }, \perp)}=-\frac{e^{2} T}{3 \pi s} \frac{\xi_{x y}^{2}}{\sqrt{\epsilon(\epsilon+r)}} .
$$

These results confirm the qualitative estimation (62) additionally providing the exact value of the numerical coefficient and the temperature dependence in the crossover region. In the limit $r \gg \epsilon$ Exp.(75) transforms into the diamagnetic susceptibility of the $3 D$ anisotropic superconductor 30].

\footnotetext{
${ }^{14}$ Let us stress the difference between $J$ and $\mathcal{J}$ in the two definitions (63) and (74) of the anisotropy parameter $r$. The first one was introduced as the electron tunneling matrix element, while the second one enters in the LD functional as the characteristic Josephson energy for the order parameter. Later on, in the framework of the microscopic theory, it will be demostrated that, in accordance with our qualitative definition, $r \sim J^{2}$, while $\mathcal{J}$ turns out to be proportional to $J^{2}$ too. In the dirty case it depends on the relaxation time of the electron scattering on impurities: $\mathcal{J} \sim \alpha J^{2} \max \{\tau, 1 / T\}$. Hence both definitions (63), appearing in the qualitative consideration, and (74), following from the LD model, are consistent.
} 
For a film of thickness $d$ the integral over $k_{z}$ in Exp.(73) has to be replaced by a summation over the discrete $k_{z}$ and when $\xi_{z}(T) \gg d$ only the term with $k_{z}=0$ has to be taken into account:

$$
\chi_{(f i l m, \perp)}=-\frac{e^{2} T}{3 \pi d} \frac{\xi_{x y}^{2}}{\epsilon} .
$$

Note that these formulas predict a nontrivial increase of diamagnetic susceptibility for clean metals [29]. The usual statement that fluctuations are most important in dirty superconductors with a short electronic mean free path does not hold in the particular case of susceptibility because here $\xi$ turns out to be in the numerator of the fluctuation correction.

Now we will demonstrate that, besides the crossovers in its temperature dependence, the fluctuation induced magnetization is a nonlinear function of magnetic field too, and these nonlinearities, different for various dimensionalities, take place at relatively low fields. This, strong in comparison with the expected scale of $H_{c 2}(0)$, manifestation of the nonlinear regime in fluctuation magnetization and hence, field dependent fluctuation susceptibility, was the subject of the intensive debates in early seventies [30, 31, 32, 33, 34, 35, 36, 37, 38, 39, (see also the old but excellent review of W.J. Skocpol and M. Tinkham 40]) and after the discovery of HTS 41, 42, 43, 44] ( see also very recent detailed essay of T.Mishonov and E.Penev [45] with references there). We will mainly follow here the paper of Buzdin et al. [46], dealing with the fluctuation magnetization of a layered superconductor, which permits observing in a unique way all variety of the crossover phenomena in temperature and magnetic field.

Let us go back to the general expression (72) and evaluate it without taking the magnetic field to small. The difficulty in dealing with it consists in the divergence of the sum over Landau levels $n$. This divergence can be regularized (see [45, 177]), but let us observe that in order to calculate the magnetization we must know the magnetic field dependent part of the free energy only. So a very convenient method to bypass the divergence problem 48 is to calculate the difference $F(H)-F(0)$, turning the sum over Landau states in $F(\epsilon, 0)$ into an integral and then, in its turn, turning this integral into a sum of integrals over the unit length intervals $x \in[n-1 / 2, n+1 / 2]$. Then

$$
\begin{aligned}
F(\epsilon, 0)= & -\lim _{H \rightarrow 0} \frac{2 \pi V H}{\Phi_{0}} T \int_{-\pi / s}^{\pi / s} \frac{d k_{z}}{2 \pi} \sum_{n=0}^{\infty} \int_{-1 / 2}^{1 / 2} d x \times \\
& \times \ln \frac{\pi T}{\alpha T_{c} \epsilon+\frac{H}{2 m \Phi_{0}}\left(x+n+\frac{1}{2}\right)+\mathcal{J}\left(1-\cos \left(k_{z} s\right)\right)} .
\end{aligned}
$$


and by introducing the dimensionless variable ${ }^{15}$

$$
h=\frac{H}{H_{c 2}(0)}, H_{c 2}(0)=2 m \alpha T_{c} / e=\Phi_{0} / 2 \pi \xi_{x y}^{2},
$$

one can write

$$
\begin{aligned}
& F(\epsilon, H)-F(\epsilon, 0)= \\
= & -\frac{T V}{2 \pi s \xi_{x y}^{2}} h \int_{-\pi}^{\pi} d z \sum_{n=0} \int_{-1 / 2}^{1 / 2} d x \ln \frac{(2 n+1+2 x) h+r / 2(1-\cos z)+\epsilon}{(2 n+1) h+r / 2(1-\cos z)+\epsilon} .
\end{aligned}
$$

Performing the integrations over $z$ and $x$ in $(78)$ and differentiating with respect to $h$ we finally obtain a very convenient general expression for the fluctuation magnetization in a layered superconductor:

$$
\begin{aligned}
M(\epsilon, H)=\frac{T}{\Phi_{0} s} & \sum_{n=0}^{\infty}\left\{n \ln \frac{\varphi\left(R_{n}+1\right)}{\varphi\left(R_{n}\right)}+\right. \\
& \left.\ln \frac{\varphi\left(R_{n}+1\right)}{\varphi\left(R_{n}+1 / 2\right)}-\frac{n+1 / 2}{\sqrt{\left(R_{n}+1 / 2\right)^{2}-\rho^{2}}}\right\}
\end{aligned}
$$

with $\varphi(x)=x+\sqrt{x^{2}-\rho^{2}}, R_{n}=n+\epsilon / 2 h+\rho$ and $\rho=r / 2 h$. The sum in (79) converges as $1 / n^{2}$ and it provides a volume magnetization expression that can be compared with experiment.

Let us comment on the different crossovers in the $M(\epsilon, H)$ field dependence analyzing the general formula (79). Let us fix the temperature $\epsilon \ll r$. In this case the $c$-axis coherence length exceeds the interlayer distance $\left(\xi_{z} \gg s\right.$ ) and in the absence of a magnetic field the fluctuation Cooper pairs motion has a $3 D$ character. Supposing the magnetic field to be not too high $(h \ll r)$ we may perform an expansion in (79) in1/ $\rho$ and obtain

$$
\begin{aligned}
& M_{(3)}(\epsilon \ll r, h \ll r)=\frac{T \sqrt{2} h^{1 / 2}}{\Phi_{0} \xi_{z}} \times \\
& \sum_{n}\left\{(n+1)\left(n+1+\frac{\epsilon}{2 h}\right)^{1 / 2}-\right. \\
&\left.-n\left(n+\frac{\epsilon}{2 h}\right)^{1 / 2}-\frac{3}{2} \frac{\left(n+1+\frac{2 \epsilon}{3 h}\right)}{\sqrt{n+\frac{1}{2}+\frac{\epsilon}{2 h}}}\right\} .
\end{aligned}
$$

For weak fields $(h \ll \epsilon)$ the magnetization grows linearly with magnetic field, justifying our preliminary qualitative results. Nevertheless, this linear

$\overline{15}$ Let us remind that the exact definition of $H_{c 2}(0)$ contains the numerical coefficient $A(0)$ (see footnote 3 ). 
growth is changed to the nonlinear $3 D$ high field regime $M \sim \sqrt{H}$ already in the region of a relatively small fields $H_{c 2}(\epsilon) \lesssim H(\epsilon \lesssim h)$. The further increase of magnetic field leads to the next crossover in the magnetization field dependence at $h \sim r$. However the Exp.(80) was obtained in the assumption $h \ll r$ as an expansion over $1 / \rho$, and it does not work any more. Handling with the Hurvitz zeta-function the summation in $(79)$ for $3 D$ case can be carried out for an arbitrary field [34:

$$
\begin{aligned}
& M_{(3)}(\epsilon \ll r, h)=3 \frac{T}{\Phi_{0} s}\left(\frac{2}{r}\right)^{1 / 2} \sqrt{h} \times \\
& {\left[\zeta\left(-\frac{1}{2}, \frac{1}{2}+\frac{\epsilon}{2 h}\right)-\zeta\left(\frac{1}{2}, \frac{1}{2}+\frac{\epsilon}{2 h}\right) \frac{\epsilon}{6 h}\right] . }
\end{aligned}
$$

One can see from this formula that for large fields the magnetization saturates at the value $M_{\infty}[49$ :

$$
M(h \gg r) \rightarrow M_{\infty}=-\frac{\ln 2}{2} \frac{T}{\Phi_{0} s}=-0.346 \frac{T}{\Phi_{0} s},
$$

that is a typical for $2 D$ superconductors. Therefore at $h \sim r$ we have a $3 D \rightarrow 2 D$ crossover in $M(H)$ behavior in spite of the fact that all sizes of fluctuation Cooper pair exceed considerably the lattice parameters. The effective "bidimensionalization" of the fluctuations is related to the effect of a strong magnetic field which "freezes out" the rotations along its direction. Let us stress that this crossover occurs in the region of already strongly nonlinear dependence of $M(H)$ and therefore for a rather strong magnetic field from the experimental point of view in HTS.

Fixing the temperature $\epsilon \gg r$ in the formula (79) one can find the general formula for $2 D$ fluctuation regime [45]:

$$
\begin{gathered}
M_{(2)}(\epsilon \gg r, h)=\frac{T}{\Phi_{0} s}\left\{\ln \Gamma\left(\frac{1}{2}+\frac{\epsilon}{2 h}\right)-\frac{1}{2} \ln (2 \pi)\right. \\
\left.-\frac{\epsilon}{2 h}\left[\psi\left(\frac{1}{2}+\frac{\epsilon}{2 h}\right)-1\right]\right\},
\end{gathered}
$$

where $\Gamma(x)$ is the Euler gamma-function and $\psi(x)=d \ln \Gamma(x) / d x$ is already cited in Introduction digamma-function. Using (83) one can directly pass from the linear regime in a weak magnetic field corresponding to (60) to the saturation of magnetization 82 in strong fields.

Near the line of the upper critical field $\left(h_{c 2}(\epsilon)=-\epsilon\right)$ the contribution of the term with $n=0$ in the sum (79) becomes most important and for the magnetization the expression

$$
-M(h) \sim \frac{h}{\sqrt{\left(h-h_{c 2}\right)\left(h-h_{c 2}+r\right)}}
$$




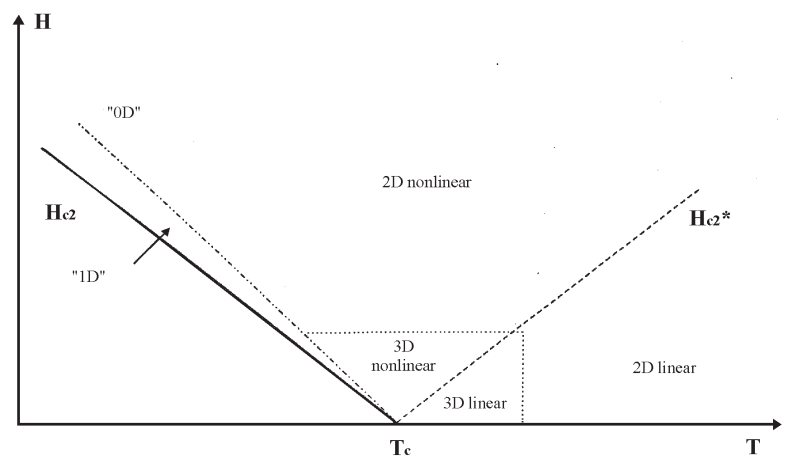

Fig. 3. Schematic representation of the different regimes for fluctuation magnetization in the $(\mathrm{H}, \mathrm{T})$ diagram. The line $H_{c 2}^{*}(T)$ is mirror-symmetric to the $H_{c 2}(T)$ line with respect to a $y$-axis passing through $T=T_{c}$. This line defines the crossover between linear and non-linear behavior of the fluctuation magnetization above $T_{c}$.

can be obtained. It contains the already familiar for us " $0 D$ " regime $(r \ll$ $\left.h-h_{c 2} \ll 1\right)$, where the magnetization decreases as $-M(h) \sim \frac{1}{h-h_{c 2}}$ (compare with (67)), while for $h-h_{c 2}<<r$ the regime becomes " $1 D$ " and the magnetization decreases slower, as $-M(h) \sim \frac{1}{\sqrt{h-h_{c 2}}}$.

Such an analogy is observed in the next orders in Gi too. In the Ref. [50] the analogy was demonstrated for the example of the first eleven terms for the $2 D$ case and nine for the $3 D$ case. Summation of the series of high order fluctuation contributions to the heat capacity by the Pade-Borel method resulted in its temperature dependence similar to the $0 D$ and $1 D$ cases without magnetic field. Nevertheless a considerable difference has not be forgotten: in the $0 D$ and $1 D$ cases no phase transition takes place while in the $2 D$ and $3 D$ cases in a magnetic field a phase transition of first order to the Abrikosov vortex lattice state occurs.

In conclusion, the fluctuation magnetization of a layered superconductor in the vicinity of the transition temperature turns out to be a complicated function of temperature and magnetic field, and it evidently cannot be factorized on these variables. The fit of the experimental data is very sensitive to the anisotropy parameter $r$ and allows determination of the latter with a rather high precision [51,52]. In Fig. 田 the successful application of the described approach to fit the experimental data on $\mathrm{YBa}_{2} \mathrm{Cu}_{3} \mathrm{O}_{7}$ is shown [53.

\section{Ginzburg-Landau theory of fluctuations in transport phenomena}

The appearance of fluctuating Cooper pairs above $T_{c}$ leads to the opening of a "new channel" for charge transfer. In the Introduction the fluctuation 


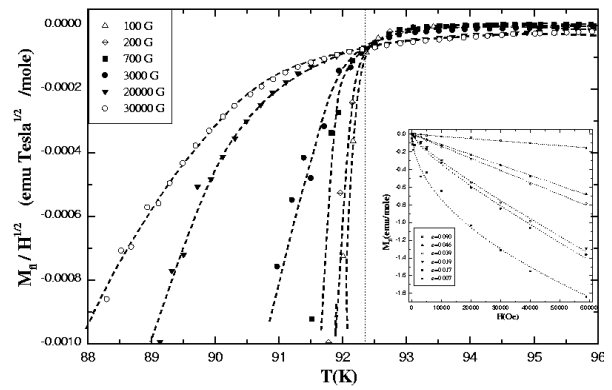

Fig. 4. Fluctuation magnetization of a YBaCO123 normalized on $\sqrt{H}$ as the function of temperature in accordance with the described theory shows the crossing of the isofield curves at $T=T_{c}(0)=92.3 \mathrm{~K}$. The best fit obtained for anisotropy parameter $r=0.09$. In the inset the magnetization curves as the function of magnetic field are reported.

Cooper pairs were treated as carriers with charge $2 e$ while their lifetime $\tau_{G L}$ was chosen to play the role of the scattering time in the Drude formula. Such a qualitative consideration results in the Aslamazov-Larkin (AL) pair contribution to conductivity (11) (so-called paraconductivity ${ }^{16}$ ).

Below we will present the generalization of the phenomenological GL functional approach to transport phenomena. Dealing with the fluctuation order parameter, it is possible to describe correctly the paraconductivity type fluctuation contributions to the normal resistance and magnetoconductivity, Hall effect, thermoelectric power and thermal conductivity at the edge of transition. Unfortunately the indirect fluctuation contributions are beyond the possibilities of the description by Time-Dependent GL approach and they will be calculated in the framework of the microscopic theory (see Sections 6-8).

\subsection{Time dependent GL equation}

In previous Sections we have demonstrated how the GL functional formalism allows one to account for fluctuation corrections to thermodynamical quantities. Let us discuss the effect of fluctuations on the transport properties of a superconductor above the critical temperature.

In order to find the value of paraconductivity, some time-dependent generalization of the GL equations is required. Indeed, the conductivity characterizes the response of the system to the applied electric field. It can be defined as $\mathbf{E}=-\partial \mathbf{A} / \partial t$ but, in contrast to the previous Section, $\mathbf{A}$ has to

$\overline{16}$ This term may have different origins. First of all, evidently, paraconductivity is analogous to paramagnetism and means excess conductivity. Another possible origin is an incorrect onomatopoeic translation from the Russian "paroprovodimost'" that means pair conductivity 
be regarded as being time dependent. The general non-stationary BCS equations are very complicated, even in the limit of slow time and space variations of the field and the order parameter. For our purposes it will be sufficient, following 66, 67, 68, 69, 70,71, 72, to write a model equation in the vicinity of $T_{c}$, which in general correctly reflects the qualitative aspects of the order parameter dynamics and in some cases is exact.

Let us keep in mind the GL functional formalism introduced above. If a deviation from equilibrium is assumed, then it is no more possible to derive the GL equations starting from the condition that the variational derivative of the free energy is zero. At the same time, in the absence of equilibrium $\Psi$ begins to depend on time. For small deviations from the equilibrium it is natural to assume that in the process of order parameter relaxation its time derivative $\partial \Psi / \partial t$ is proportional to the variational derivative of the free energy $\delta \mathcal{F} / \delta \Psi^{*}$, which is equal to zero at the equilibrium. But this is not all: side by side with the normal relaxation of the order parameter the effect of thermodynamical fluctuations on it has to be taken into account. This can be done by the introduction the Langevin forces $\zeta(\mathbf{r}, t)$ in the right-hand side of equation describing the order parameter dynamics. Finally, gauge invariance requires that $\partial \Psi / \partial t$ should be included in the equation in the combination $\partial \Psi / \partial t+2 i e \varphi \Psi$, where $\varphi$ is the scalar potential of the electric field. By concluding all these speculations one can write the model timedependent GL equation (TDGL) in the form

$$
-\gamma_{G L}\left(\frac{\partial}{\partial t}+2 i e \varphi\right) \Psi=\frac{\delta \mathcal{F}}{\delta \Psi^{*}}+\zeta(\mathbf{r}, t)
$$

with the GL functional $\mathcal{F}$ determined by $(16),(65),(69)$. The dimensionless coefficient $\gamma_{G L}$ in the left-hand-side of the equation can be related to pair life-time $\tau_{G L}$ (2): $\gamma_{G L}=\alpha T_{c} \epsilon \tau_{G L}=\pi \alpha / 8$ by the substitution in (84) of the first term of 116$)$ only 17 .

Neglecting the fourth order term in the GL functional, equation (84) can be rewritten in operator form as

$$
\left[\widehat{L}^{-1}-2 i e \gamma_{G L} \varphi(r, t)\right] \Psi(\mathbf{r}, t)=\zeta(\mathbf{r}, t)
$$

with the TDGL operator $\widehat{L}$ and Hamiltonian $\widehat{\mathcal{H}}$ defined as

$$
\widehat{L}=\left[\gamma_{G L} \frac{\partial}{\partial t}+\widehat{\mathcal{H}}\right]^{-1}, \widehat{\mathcal{H}}=\alpha T_{c}\left[\epsilon-\widehat{\xi}^{2}(\widehat{\nabla}-2 i e \mathbf{A})^{2}\right] .
$$

${ }^{17}$ It will be shown below that taking into account the electron-hole asymmetry leads to the appearance of an imaginary part of $\gamma_{G L}$ proportional to the derivative $\partial \ln \left(\rho v^{2} \tau\right) /\left.\partial E\right|_{E_{F}} \sim \mathcal{O}\left(1 / E_{F}\right)$. This is important for such phenomena as fluctuation Hall effect or fluctuation thermopower and, having in mind writing the most general formula, we will suppose $\gamma_{G L}=\pi \alpha / 8+i \operatorname{Im} \gamma_{G L}$, where necessary. 
We have introduced here the formal operator of the coherence length $\widehat{\xi}$ to have the possibility to deal with an arbitrary type of spectrum. For example, in the most interesting case for our applications to layered superconductors, the action of this operator is defined by the Exp.(69).

In the absence of an electric field one can write the formal solution of equation (85) as

$$
\Psi^{(0)}(\mathbf{r}, t)=\widehat{L} \zeta(\mathbf{r}, t) .
$$

The Langevin forces introduced above must satisfy the fluctuation-dissipation theorem, which means that the correlator $<\Psi_{\mathbf{p}}^{(0) *}\left(t^{\prime}\right) \Psi_{\mathbf{p}}^{(0)}(t)>$ at coinciding moments of time has to be the same as $\left\langle\left|\Psi_{\mathbf{p}}\right|^{2}\right\rangle$, obtained by averaging over fluctuations in thermal equilibrium. This requirement is fulfilled if the Langevin forces $\zeta(\mathbf{r}, t)$ and $\zeta^{*}(\mathbf{r}, t)$ are correlated by the Gaussian white-noise law

$$
<\zeta^{*}(\mathbf{r}, t) \zeta\left(\mathbf{r}^{\prime}, t^{\prime}\right)>=2 T \operatorname{Re} \gamma_{G L} \delta\left(\mathbf{r}-\mathbf{r}^{\prime}\right) \delta\left(t-t^{\prime}\right) .
$$

To show it let us restrict ourselves for sake of simplicity to the case of $A=0$ and calculate the correlator

$$
\begin{aligned}
& <\Psi^{*}(\mathbf{r}, t) \Psi\left(\mathbf{r}^{\prime}, t\right)>=<\zeta^{*}(\mathbf{r}, t) \widehat{\widehat{L^{*}}} \widehat{L} \zeta\left(\mathbf{r}^{\prime}, t\right)>= \\
& =2 T \operatorname{Re} \gamma_{G L} \int \frac{d \mathbf{p}}{(2 \pi)^{D}} e^{i \mathbf{p}\left(\mathbf{r}-\mathbf{r}^{\prime}\right)} \int_{-\infty}^{\infty} \frac{d \Omega}{2 \pi} L^{*}(\mathbf{p}, \Omega) L(\mathbf{p}, \Omega) .
\end{aligned}
$$

$L(\mathbf{p}, \Omega)$ can be found by making the Fourier transform in 86 ):

$$
L(\mathbf{p}, \Omega)=\left(-i \gamma_{G L} \Omega+\varepsilon_{\mathbf{p}}\right)^{-1},
$$

resulting in

$$
\left\langle\Psi^{*}(\mathbf{r}, t) \Psi\left(\mathbf{r}^{\prime}, t\right)\right\rangle_{\mathbf{p}}=2 T \operatorname{Re} \gamma_{G L} \int_{-\infty}^{\infty} \frac{d \Omega}{2 \pi} \frac{1}{\gamma_{G L}^{2} \Omega^{2}+\varepsilon_{\mathbf{p}}^{2}}=\left\langle\left|\Psi_{\mathbf{p}}\right|^{2}\right\rangle,
$$

where

$$
\varepsilon_{\mathbf{p}}=\alpha T_{c}\left(\epsilon+\widehat{\xi}^{2} \mathbf{p}^{2}\right) .
$$

is the Cooper pair energy spectrum.

\subsection{Paraconductivity}

Let us try to clear up the reason why the simple Drude formula works so well for complex phenomenon like paraconductivity. For this purpose let us try to derive the Boltzmann master equation for the fluctuation Cooper pair distribution function

$$
n_{\mathbf{p}}(t)=\int\left\langle\Psi(\mathbf{r}, t) \Psi^{*}\left(\mathbf{r}^{\prime}, t\right)\right\rangle \exp \left(-i \mathbf{p}\left(\mathbf{r}-\mathbf{r}^{\prime}\right)\right) d\left(\mathbf{r}-\mathbf{r}^{\prime}\right)
$$


Let us recall that in the state of thermal equilibrium $n_{\mathbf{p}}^{(0)}=<\left|\Psi_{\mathbf{p}}\right|^{2}>=T / \varepsilon_{\mathbf{p}}$.

In order to determine the electric field dependence of $n_{\mathbf{p}}$ let us write its time derivative using (84)

$$
\begin{aligned}
\frac{\partial n_{\mathbf{p}}(t)}{\partial t}= & \int d\left(\mathbf{r}-\mathbf{r}^{\prime}\right) e^{-i \mathbf{p}\left(\mathbf{r}-\mathbf{r}^{\prime}\right)}\left[\left\langle\frac{\partial \Psi(\mathbf{r}, t)}{\partial t} \Psi^{*}\left(\mathbf{r}^{\prime}, t\right)\right\rangle+\left\langle\Psi\left(\mathbf{r}^{\prime}, t\right) \frac{\partial \Psi^{*}(\mathbf{r}, t)}{\partial t}\right\rangle\right]= \\
= & \int d\left(\mathbf{r}-\mathbf{r}^{\prime}\right) e^{-i \mathbf{p}\left(\mathbf{r}-\mathbf{r}^{\prime}\right)}\left[2 \frac{e}{i}\left[\varphi(\mathbf{r})-\varphi\left(\mathbf{r}^{\prime}\right)\right]<\Psi(\mathbf{r}, t) \Psi^{*}\left(\mathbf{r}^{\prime}, t\right)>+\right. \\
& \left.+\frac{2}{\gamma_{G L}} \operatorname{Re}\left\langle\Psi(\mathbf{r}, t) \frac{\delta \mathcal{F}}{\delta \Psi}\left(\mathbf{r}^{\prime}, t\right)\right\rangle+\frac{2}{\gamma_{G L}} \operatorname{Re}\left\langle\zeta(\mathbf{r}, t) \Psi^{*}\left(\mathbf{r}^{\prime}, t\right)\right\rangle\right],
\end{aligned}
$$

where $\mathcal{F}$ is determined by $(65)$. Expressing the scalar potential by the electric field $E$ one can transform the first term of the last integral into $-2 e \mathbf{E} \frac{\partial n_{\mathbf{p}}}{\partial \mathbf{p}}$. The term with the variational derivative can be evaluated by means of (26) and expressed in the form $-\frac{2}{\gamma_{G L}} \varepsilon_{\mathbf{p}} n_{\mathbf{p}}$. More cumbersome is the evaluation of the last term, containing the Langevin force. To the first approximation it is possible to use here the order parameter $\Psi^{(0)}(\mathbf{r}, t)$ (see (87)) unperturbed by the electric field as the convolution $\widehat{L} \zeta(\mathbf{r}, t)$. In this way, using (88) and (89) we calculate the last average in (94)

$$
\begin{aligned}
\frac{2}{\gamma_{G L}} \int d\left(\mathbf{r}-\mathbf{r}^{\prime}\right) e^{-i \mathbf{p}\left(\mathbf{r}-\mathbf{r}^{\prime}\right)} \operatorname{Re}\left\langle\zeta^{*}(\mathbf{r}, t) \widehat{L} \zeta(\mathbf{r}, t)\right\rangle & = \\
4 T \int \frac{d \mathbf{p}}{(2 \pi)^{D}} e^{i \mathbf{p}\left(\mathbf{r}-\mathbf{r}^{\prime}\right)} \operatorname{Re} \int_{-\infty}^{\infty} \frac{d \Omega}{2 \pi} L(\mathbf{p}, \Omega) & =\frac{2 T}{\gamma_{G L}}=\frac{2}{\gamma_{G L}} \varepsilon_{\mathbf{p}} n_{\mathbf{p}}^{(0)}
\end{aligned}
$$

and obtain the transport equation

$$
\frac{\partial n_{\mathbf{p}}}{\partial t}+2 e \mathbf{E} \frac{\partial n_{\mathbf{p}}}{\partial \mathbf{p}}=-\frac{2}{\gamma_{G L}} \varepsilon_{\mathbf{p}}\left(n_{\mathbf{p}}-n_{\mathbf{p}}^{(0)}\right)=-\frac{2}{\tau_{\mathbf{p}}}\left(n_{\mathbf{p}}-n_{\mathbf{p}}^{(0)}\right) .
$$

In the absence of a magnetic field $\varepsilon_{\mathbf{p}}$ was determined by (92) and the momentum dependent lifetime, corresponding to the Ginzburg-Landau one, can be introduced:

$$
\tau_{\mathbf{p}}=\gamma_{G L} / \varepsilon_{\mathbf{p}}=\frac{\tau_{G L}(\epsilon)}{1+\xi^{2}(\epsilon) \mathbf{p}^{2}} .
$$

Let us stress the appearance of the coefficient 2 on the right hand side of the equation (95). This means that the real Cooper pair lifetime, characterizing its density decay, is $\tau_{\mathbf{p}} / 2$.

The effect of a weak electric field on the fluctuation Cooper pair distribution function in the linear approximation is determined by

$$
n_{\mathbf{p}}^{(1)}=-\frac{e \mathbf{E} \gamma_{G L}}{\varepsilon_{\mathbf{p}}} \frac{\partial n_{\mathbf{p}}^{(0)}}{\partial \mathbf{p}}=\frac{e T \gamma_{G L}}{\varepsilon_{\mathbf{p}}^{3}} \mathbf{E} \cdot \frac{\partial \varepsilon_{\mathbf{p}}}{\partial \mathbf{p}} .
$$


Substituting this formula into the expression for the electric current side by side with the Cooper pair velocity $\mathbf{v}_{\mathbf{p}}=\partial \varepsilon_{\mathbf{p}} / \partial \mathbf{p}$ one can find

$$
\mathbf{j}^{\alpha}=\sum_{\mathbf{p}}\left(2 e \mathbf{v}^{\alpha} n_{\mathbf{p}}\right)=\sigma^{\alpha \beta} E^{\beta},
$$

where the paraconductivity tensor components are:

$$
\sigma_{(D)}^{\alpha \beta}=\frac{\pi}{4} e^{2} \alpha T \sum_{\mathbf{p}} \frac{\mathbf{v}_{\mathbf{p}}^{\alpha} \mathbf{v}_{\mathbf{p}}^{\beta}}{\varepsilon_{\mathbf{p}}^{3}} .
$$

In the case of isotropic spectrum

$$
\sigma_{(D)}^{\alpha \beta}=\frac{\pi}{4} e^{2} \alpha T \sum_{\mathbf{p}} \frac{\mathbf{v}_{\mathbf{p}}^{\alpha} \mathbf{v}_{\mathbf{p}}^{\beta}}{\varepsilon_{\mathbf{p}}^{3}}= \begin{cases}\frac{e^{2}}{32 \xi} \frac{1}{\sqrt{\epsilon}} & \text { 3D case, } \\ \frac{e^{2}}{16 d} \frac{1}{\epsilon} & \text { 2D film, thickness }: d \ll \xi, \\ \frac{\pi e^{2} \xi}{16 S} \frac{1}{\epsilon^{3 / 2}} & 1 \mathrm{D} \text { wire, cross }- \text { section }: S \ll \xi^{2} .\end{cases}
$$

One can compare this result with that carried out in the Introduction from qualitative consideration, based on the Drude formula. Those simple speculations reflect correctly the physics of the phenomenon but were carried out with the assumption of the momentum independence of the relaxation time $\tau_{\mathbf{p}}$, taken as $\tau_{\mathbf{0}}=\frac{\pi}{8\left(T-T_{c}\right)}$. As we have just seen, in reality $\tau_{\mathbf{p}}$ decreases rapidly with increase of the momentum, the excess "2" appeared in (95) because of the wave nature of fluctuation Cooper pairs; accounting for this circumstance results in the precise coefficients of (99), different from (11).

An especially simple form the paraconductivity results in the $2 D$ case, where, calculated per unit square, it depends on the reduced temperature only:

$$
\sigma_{\square}(T)=\frac{e^{2}}{16 \hbar} \frac{T}{T-T_{c}} .
$$

The coefficient in this formula turns out to be a universal constant and is given by the value $\hbar / e^{2}=4.1 k \Omega$. For electronic spectra of other dimensionalities this universality is lost, and the paraconductivity comes to depend on the electron mean free path.

Let us compare $\sigma_{\square}(T)$ with the normal electron Drude part $\sigma_{n}=n_{e} e^{2} \tau / m$ by writing the total conductivity

$$
\sigma=\frac{e^{2}}{\hbar}\left(\frac{p_{F} l}{2 \pi \hbar}+\frac{1}{16 \epsilon}\right)
$$

One sees that at $\epsilon_{c r}=0.4 /\left(p_{F} l\right) \sim G i_{(2 c)}$ the fluctuation correction reaches the value of the normal conductivity. Let us recall that the same order of 
magnitude for the $2 D$ Ginzburg-Levanyuk number was obtained above from the heat capacity study. We will discuss the region of applicability of (101) below in Section 8.6.

It is worth mentioning that the results derived here for paraconductivity are valid with the assumption of weak fluctuations: for the temperature range $\epsilon \lesssim G i_{(D)}$ they are not anymore applicable. Nevertheless, one can see that for not very dirty films, with $p_{F}^{2} l d \gg 1$, a wide region of temperatures $G i_{(2 d)} \ll$ $\epsilon \ll 1$ exists where the temperature dependence of conductivity is determined by fluctuations and in this region the localization effects are negligible.

The transport equation (95) was originally derived many years ago by L.G.Aslamazov and A.I.Larkin [73]. Recently T.Mishonov et al. [74] rederived Eq.95) and solved it for $n_{\mathbf{p}}$ in the case of an arbitrary electric field.

\subsection{General expression for paraconductivity}

Unfortunately the applicability of the master equation derived is restricted to weak magnetic fields $\left(H \ll H_{c 2}(\epsilon)\right)$. For stronger fields $H_{c 2}(\epsilon) \lesssim H \ll$ $H_{c 2}(0)$ the simple evaluation of averages in (94) turns out to be incorrect, the density matrix has to be introduced and the master equation loses its attractive simplicity. At the same time, as we already know, namely these fields, quantizing the fluctuation Cooper pair motion, present special interest. This is why in order to include in the scheme the magnetic field and frequency dependencies of the paraconductivity, we return to the analysis of the general TDGL equation (84) without the objective to reduce it to a Boltzmann type transport equation.

Let us solve it in the case when the applied electric field can be considered as a perturbation. The method will much resemble an exercise from a course of quantum mechanics. To carry out the necessary generality side by side with a formal simplicity of expressions we will introduce some kind of subscript $\{i\}$ which includes the complete set of quantum numbers and time. By a repeated subscript a summation over a discrete and integration over continuous variables (time in particular) will be supposed.

We will look for the response of the order parameter to a weak electric field applied in the form

$$
\Psi_{k_{z}}(\mathbf{r}, t)=\Psi_{\{i\}}^{(0)}+\Psi_{\{i\}}^{(1)},
$$

where $\Psi_{\{i\}}^{(0)}$ is determined by (87). Substituting this expression into 85) and restricting our consideration to linear terms in the electric field we can write

$$
\left(\widehat{L}^{-1}\right)_{\{i k\}} \Psi_{\{k\}}^{(1)}=2 i e \gamma_{G L} \varphi_{\{i l\}} \Psi_{\{l\}}^{(0)}
$$

with the solution in the form

$$
\Psi_{\{i\}}^{(1)}=2 i e \gamma_{G L} \widehat{L}_{\{i k\}} \varphi_{\{k l\}} \widehat{L}_{\{l m\}} \zeta_{\{m\}} .
$$


Let us substitute the order parameter (102) in the quantum mechanical expression for current

$$
\mathbf{j}=2 e \operatorname{Re}\left[\Psi_{\{i\}}^{(0) *} \widehat{\mathbf{v}}_{\{i k\}} \Psi_{\{k\}}^{(1)}+\Psi_{\{i\}}^{(1) *} \widehat{\mathbf{v}}_{\{i k\}} \Psi_{\{k\}}^{(0)}\right],
$$

where $\widehat{\mathbf{v}}_{\{i k\}}$ is the velocity operator which can be expressed by means of the commutator of $\mathbf{r}$ with Hamiltonian (86):

$$
\widehat{\mathbf{v}}_{\{i k\}}=i\{\widehat{\mathcal{H}}, \mathbf{r}\}_{\{i k\}} .
$$

The second term of (104) can be written by means of a transposed velocity operator (which is Hermitian) as the complex conjugated value of the first one:

$$
\Psi_{\{i\}}^{(1) *} \widehat{\mathbf{v}}_{\{i k\}} \Psi_{\{k\}}^{(0)}=\left(\Psi_{\{k\}}^{(0) *} \widetilde{\mathbf{v}}_{\{i k\}} \Psi_{\{i\}}^{(1)}\right)^{*},
$$

which results in

$$
\begin{aligned}
\mathbf{j} & =2 \operatorname{Re}\left\{\Psi_{\{i\}}^{(0) *}\left(2 e \widehat{\mathbf{v}}_{\{i k\}}\right) \Psi_{\{k\}}^{(1)}\right\}= \\
& =-8 e^{2} \operatorname{Im}\left\{\gamma_{G L} \widehat{L}_{\{k i\}}^{*} \widehat{\mathbf{v}}_{\{i l\}} \widehat{L}_{\{l m\}} \varphi_{\{m n\}} \widehat{L}_{\{n p\}} \zeta_{\{k\}}^{*} \zeta_{\{p\}}\right\} .
\end{aligned}
$$

Let us average now (107) over the Langevin forces moving the operator $\widehat{L}_{\{k i\}}^{*}$ from the beginning to the end of the trace and using (88). One finds

$$
\mathbf{j}=-16 T e^{2} \operatorname{Re}\left(\gamma_{G L}\right) \operatorname{Im}\left\{\gamma_{G L} \widehat{\mathbf{v}}_{\{i l\}} \widehat{L}_{\{l m\}} \varphi_{\{m n\}} \widehat{L}_{\{n p\}} \widehat{L}_{\{p i\}}^{*}\right\} .
$$

Now we choose the representation where the $\widehat{L}_{\{l m\}}$ operator is diagonal (it is evidently given by the eigenfunctions of the Hamiltonian (86)):

$$
L_{\{m\}}(\Omega)=\frac{1}{\varepsilon_{\{m\}}-i \Omega \gamma_{G L}},
$$

where $\varepsilon_{\{m\}}$ are the appropriate energy eigenvalues. Then we assume that the electric field is coordinate independent but is a monochromatic periodic function of time:

$$
\varphi(r, t)=-E^{\beta} r^{\beta} \exp (-i \omega t) .
$$

In doing the Fourier transform in (108) one has to remember that the time dependence of the matrix elements $\varphi_{\{m n\}}$ results in a shift of the frequency variable of integration $\Omega \rightarrow \Omega-\omega$ in both L-operators placed after $\varphi_{\{m n\}}$ or, what is the same, to a shift of the argument of the previous $\widehat{L}_{\{l m\}}$ for $\omega$ :

$$
\begin{aligned}
\mathbf{j}_{\omega}^{\alpha}= & 16 T e^{2} \operatorname{Re}\left(\gamma_{G L}\right) \times \\
& \times \int \frac{d \Omega}{2 \pi} \Re\left\{\gamma_{G L} \widehat{\mathbf{v}}_{\{i l\}}^{\alpha} \widehat{L}_{\{l\}}(\Omega+\omega)\left[-i r_{\{l i\}}^{\beta}\right] \widehat{L}_{\{i\}}(\Omega) \widehat{L}_{\{i\}}^{*}(\Omega)\right\} \mathbf{E}^{\beta},
\end{aligned}
$$


where $\Re f(\omega) \equiv\left[f(\omega)+f^{*}(-\omega)\right] / 2$.

Let us express the matrix element $\mathbf{r}_{\{l i\}}$ by means of $\widehat{\mathbf{v}}_{\{l i\}}$ using the commutation relation (105). One can see that in the representation chosen

$$
\widehat{\mathbf{r}}_{\{l i\}}^{\beta}=i \frac{\widehat{\mathbf{v}}_{\{l i\}}^{\beta}}{\varepsilon_{\{i\}}-\varepsilon_{\{l\}}}
$$

and, carrying out the frequency integration in (111), finally write for the fluctuation conductivity tensor $\left(\mathbf{j}_{\omega}^{\alpha}=\sigma^{\alpha \beta}(\omega) \mathbf{E}^{\beta}\right)$ :

$$
\begin{aligned}
\sigma^{\alpha \beta}(\epsilon, H, \omega)= & 8 e^{2} T \operatorname{Re}\left(\gamma_{G L}\right) \sum_{\{i, l\}=0}^{\infty} \\
& \Re\left[\gamma_{G L} \frac{\widehat{\mathbf{v}}_{\{i l\}}^{\alpha} \widehat{\mathbf{v}}_{\{l i\}}^{\beta}}{\varepsilon_{\{i\}}\left(\gamma_{G L} \varepsilon_{\{i\}}+\gamma_{G L}^{*} \varepsilon_{\{l\}}-i\left|\gamma_{G L}\right|^{2} \omega\right)\left(\varepsilon_{\{l\}}-\varepsilon_{\{i\}}\right)}\right] .
\end{aligned}
$$

This is the most general expression which describes the d.c., galvanomagnetic and high frequency paraconductivity contribution. In the case when we are interested in diagonal effects only, where it is enough to accept $\gamma_{G L}$ as real $\left(\gamma_{G L}=\operatorname{Re} \gamma_{G L}=\pi \alpha / 8\right)$ omitting its small imaginary part, the last expression can be simplified by means of symmetrization of the summation variables):

$$
\sigma^{\alpha \alpha}(\epsilon, H, \omega)=\frac{\pi}{2} \alpha e^{2} T \sum_{\{i, l\}=0}^{\infty} \Re\left[\frac{\widehat{\mathbf{v}}_{\{i l\}}^{\alpha} \widehat{\mathbf{v}}_{\{l i\}}^{\alpha}}{\varepsilon_{\{i\}} \varepsilon_{\{l\}}\left(\varepsilon_{\{i\}}+\varepsilon_{\{l\}}-i \gamma_{G L} \omega\right)}\right] .
$$

Let us demonstrate the calculation of the d.c. paraconductivity in the simplest case of a metal with an isotropic spectrum. In this case we choose a plane wave representation. By using $\varepsilon_{\mathbf{p}}$ defined by (92) one has

$$
\widehat{\mathbf{v}}_{\left\{\mathbf{p p}^{\prime}\right\}}=\mathbf{v}_{\mathbf{p}} \delta_{\mathbf{p p}^{\prime}}, \mathbf{v}_{\mathbf{p}}=\frac{\partial \varepsilon_{\mathbf{p}}}{\partial \mathbf{p}}=2 \alpha T_{c} \xi^{2} \mathbf{p} .
$$

We do not need to keep the imaginary part of $\gamma_{G L}$, which is necessary to calculate particle-hole asymmetric effects only. Then the fluctuation conductivity calculated from (114) coincides exactly with (98).

\subsection{Fluctuation conductivity of layered superconductor}

Let us return to the discussion of our general formula (114) for the fluctuation conductivity tensor. A magnetic field directed along the c-axis still permits separation of variables even in the case of a layered superconductor. The Hamiltonian in this case can be written as in (71), (86):

$$
\widehat{\mathcal{H}}=\alpha T_{c}\left(\epsilon-\xi_{x y}^{2}\left(\nabla_{x y}-2 i e \mathbf{A}_{x y}\right)^{2}-\frac{r}{2}\left(1-\cos \left(k_{z} s\right)\right) .\right.
$$


It it is convenient to work in the Landau representation, where the summation over $\{i\}$ is reduced to one over the ladder of the Landau levels $i=0,1,2$.. (each is degenerate with a density $2 e H$ per unit square) and integration over the c-axis momentum in the limits of the Brillouine zone. The eigenvalues of the Hamiltonian (116) can be written in the form

$$
\varepsilon_{n}=\alpha T_{c}\left[\epsilon+\frac{r}{2}\left(1-\cos \left(k_{z} s\right)\right)+h(2 n+1)\right]=\varepsilon_{k_{z}}+\alpha T_{c} h(2 n+1),
$$

where $h=\frac{e H}{2 m \alpha T_{c}}$ was already defined by Eq. (77). For the velocity operators one can write

$$
\widehat{\mathbf{v}}^{x, y}=\frac{1}{2 m}(-i \nabla-2 i e \mathbf{A})^{x, y} ; \widehat{\mathbf{v}}^{z}=-\frac{\alpha r s}{2} T_{c} \sin \left(k_{z} s\right) .
$$

In-plane conductivity. Let us start from the calculation of the in-plane components. The calculation of the velocity operator matrix elements requires some special consideration. First of all let us stress that the required matrix elements have to be calculated for the eigenstates of a quantum oscillator whose motion is equivalent to the motion of a charged particle in a magnetic field. The commutation relation for the oscillator's velocity components is well known (see 75):

$$
\left[\widehat{\mathbf{v}}^{x}, \widehat{\mathbf{v}}^{y}\right]=i \frac{e H}{2 m^{2}}=\frac{i \alpha T_{c}}{m} h .
$$

In order to calculate the necessary matrix elements let us present the velocity operator components in the form of boson-type creation and annihilation operators $\widehat{a}^{+}, \widehat{a}$ with commutation relation $\left[\widehat{a}, \widehat{a}^{+}\right]=1$ :

$$
\widehat{\mathbf{v}}^{x, y}=\sqrt{\frac{\alpha T_{c} h}{2 m}}\left(\begin{array}{c}
\widehat{a}^{+}+\widehat{a} \\
i \widehat{a}^{+}-i \widehat{a}
\end{array}\right)
$$

One can check that the correct commutation relation (119) is fulfilled. Taking into account that

$$
<l|\widehat{a}| n>=<n\left|\widehat{a}^{+}\right| l>=\sqrt{n} \delta_{n, l+1}
$$

it is seen that the only non-zero matrix elements of the velocity operator are

$$
<l\left|\widehat{\mathbf{v}}^{x, y}\right| n>=\sqrt{\frac{\alpha T_{c} h}{2 m}}\left(\begin{array}{c}
\sqrt{l} \delta_{l, n+1}+\sqrt{n} \delta_{n, l+1} \\
i \sqrt{l} \delta_{l, n+1}-i \sqrt{n} \delta_{n, l+1}
\end{array}\right) .
$$

Using these relations the necessary product of matrix elements can be calculated:

$$
<l\left|\widehat{\mathbf{v}}^{x}\right| n><n\left|\widehat{\mathbf{v}}^{x}\right| l>=\frac{\alpha T_{c} h}{2 m}\left(l \delta_{l, n+1}+n \delta_{n, l+1}\right) .
$$


Its substitution into (114) and accounting of the degeneracy of the Landau levels $2 e H=4 m \alpha T_{c} h$ gives for the diagonal component of the in-plane conductivity tensor

$$
\begin{aligned}
\sigma^{x x}(\epsilon, H, \omega) & =\frac{\pi \alpha^{2} T_{c}^{2} e^{2}}{4 m} h \sum_{\{n\}=0}^{\infty} \sum_{\{l\}=0} \Re\left[\frac{\left(l \delta_{l, n+1}+n \delta_{n, l+1}\right)}{\varepsilon_{l} \varepsilon_{n}\left(\varepsilon_{l}+\varepsilon_{n}-i \gamma_{G L} \omega\right)}\right] \\
& =\pi e^{2}\left(\alpha T_{c}\right)^{3} h^{2} \int_{-\frac{\pi}{s}}^{\frac{\pi}{s}} \frac{d k_{z}}{2 \pi} \sum_{n=0}^{\infty} \Re\left[\frac{n+1}{\varepsilon_{n+1} \varepsilon_{n}\left(\varepsilon_{n+1}+\varepsilon_{n}-i \gamma_{G L} \omega\right)}\right] .
\end{aligned}
$$

Expanding the denominator into simple fractions we reduce the problem to the calculation of the $c$-axis momentum integral, which can be carried out in the general case by use of the identity:

$$
\int_{0}^{2 \pi} \frac{d x}{2 \pi} \frac{1}{\cos x-z}=-\frac{1}{\sqrt{z^{2}-1}}
$$

valid for any complex parameter $z \neq 1$ with the proper choice of the square root branch. Using it we write the general expression for the in-plane component of the fluctuation conductivity tensor

$$
\begin{aligned}
\sigma^{x x}(\varepsilon, h, \omega)= & \frac{e^{2} h}{16 s} \sum_{n=0}^{\infty}(n+1)\left\{\frac{1}{h-i \widetilde{\omega}} \frac{1}{\sqrt{[\epsilon+h(2 n+1)][r+\epsilon+h(2 n+1)]}}+\right. \\
& \frac{1}{h+i \widetilde{\omega}} \frac{1}{\sqrt{[\epsilon+h(2 n+3)][r+\epsilon+h(2 n+3)]}}- \\
& \left.\frac{2 h}{h^{2}+\widetilde{\omega}^{2}} \frac{1}{\sqrt{[\epsilon+h(2 n+2)-i \widetilde{\omega}][r+\epsilon+h(2 n+2)-i \widetilde{\omega}]}}\right\} .
\end{aligned}
$$

where $\widetilde{\omega}=\frac{\pi \omega}{16 T_{c}}$.

Out-of plane conductivity. The situation with the out-of plane component of paraconductivity turns out to be even simpler because of the diagonal structure of the $\widehat{\mathbf{v}}_{\{i n\}}^{z}=-\frac{\alpha r s}{2} T_{c} \sin \left(k_{z} s\right) \times \delta_{\text {in }} \times \delta\left(k_{z}-k_{z^{\prime}}\right)$. Taking into account that the Landau state degeneracy $2 e H=h \xi_{x y}^{-2}$ we write

$$
\begin{aligned}
\sigma^{z z}(\epsilon, H, \omega) & =\frac{1}{2} \pi \alpha e^{2} T \sum_{\{i, l\}=0}^{\infty} \Re\left[\frac{\widehat{\mathbf{v}}_{\{i l\}}^{\alpha} \widehat{\mathbf{v}}_{\{l i\}}^{\alpha}}{\varepsilon_{\{i\}} \varepsilon_{\{l\}}\left(\varepsilon_{\{i\}}+\varepsilon_{\{l\}}-i \alpha T_{c} \widetilde{\omega}\right)}\right]= \\
& =\frac{\pi e^{2}\left(\alpha T_{c}\right)^{3}}{16}\left(\frac{s r}{\xi_{x y}}\right)^{2} h \sum_{n=0}^{\infty} \int_{-\frac{\pi}{s}}^{\frac{\pi}{s}} \frac{d k_{z}}{2 \pi} \Re\left[\frac{\sin ^{2}\left(k_{z} s\right)}{\varepsilon_{n}^{2}\left(k_{z}\right)\left[\varepsilon_{n}\left(k_{z}\right)-i \alpha T_{c} \widetilde{\omega}\right]}\right]
\end{aligned}
$$


The following transformations are similar to the calculation of the in-plane component: we expand the integrand into simple fractions and perform the $k_{z}$-integration by means of the identity (122). The final expression can be written as

$$
\begin{aligned}
\sigma^{z z}(\epsilon, H, \omega)= & \frac{\pi e^{2}}{32 s}\left(\frac{s r}{\xi_{x y}}\right)^{2} h \sum_{n=0}^{\infty}\left(-\frac{\partial}{\partial \lambda}\right) \times \\
& \Re\left(\frac{1}{\lambda+i \widetilde{\omega}}\right)\left\{\frac{1}{\sqrt{(\epsilon+h(2 n+1)+\lambda)(\epsilon+h(2 n+1)+\lambda+r)}}-\right. \\
& \left.\frac{1}{\sqrt{(\epsilon+h(2 n+1)-i \widetilde{\omega})(\epsilon+h(2 n+1)-i \widetilde{\omega}+r)}}\right\}\left.\right|_{\lambda=0} .
\end{aligned}
$$

Analysis of the general expressions. In principle the expressions derived above give an exact solution for the a.c. $(\omega \ll T)$ paraconductivity tensor of a layered superconductor in a perpendicular magnetic field $H \ll H_{c 2}(h \ll 1)$ in the vicinity of the critical temperature $(\epsilon \ll 1)$. The interplay of the parameters $r, \epsilon, \omega h$ entering into (123)-(124), as we have seen in the example of fluctuation magnetization, yields a variety of crossover phenomena.

1. The simplest and most important results which can be derived are the components of the d.c. paraconductivity $(\omega=0)$ of layered superconductor in the absence of magnetic field. Keeping $\omega=0$ and setting $h \rightarrow 0$ one can change the summations over Landau levels into integration and find

$$
\begin{aligned}
& \sigma^{x x}(\varepsilon, h \rightarrow 0, \omega=0)=\frac{e^{2}}{16 s} \frac{1}{\sqrt{[\epsilon(r+\epsilon)]}}, \\
& \sigma^{z z}(\epsilon, h \rightarrow 0, \omega=0)=\frac{e^{2} s}{32 \xi_{x y}^{2}}\left(\frac{\epsilon+r / 2}{[\epsilon(\epsilon+r)]^{1 / 2}}-1\right) .
\end{aligned}
$$

2. The Aslamazov-Larkin contribution to the magnetoconductivity can be studied by putting $\omega=0$ and keeping magnetic field as arbitrary. We will not go into details and just report the results (following [154] with some revision of the coefficient in $3 D$ case)

\begin{tabular}{|l|l|l|l|}
\hline & $h \ll \epsilon$ & $\begin{array}{c}\epsilon \ll h \ll r \\
(3 D)\end{array}$ & $\begin{array}{c}\max \{\epsilon, r\} \ll h \\
(2 D)\end{array}$ \\
\hline$\sigma^{x x}$ & $\sigma^{x x}(\epsilon, h=0)-\frac{e^{2}}{2^{8} s} \frac{\left[8 \epsilon(\epsilon+r)+3 r^{2}\right]}{[\epsilon(\epsilon+r)]^{5 / 2}} h^{2}$ & $\frac{e^{2}}{4 s} \frac{1}{\sqrt{2 h r}}$ & $\frac{e^{2}}{8 s} \frac{1}{h}$ \\
\hline$\sigma^{z z}$ & $\sigma^{z z}(\epsilon, h=0)-\frac{e^{2} s}{2^{8} \xi_{x y}^{2}} \frac{r^{2}(\epsilon+r / 2)}{[\epsilon(\epsilon+r)]^{5 / 2}} h^{2}$ & $\frac{3.24 e^{2} s}{\xi_{x y}^{2}} \sqrt{\frac{r}{h}}$ & $\frac{7 \zeta(3) e^{2} s}{2^{9} \xi_{x y}^{2}} \frac{r^{2}}{h^{2}}$ \\
\hline
\end{tabular}

Table 2. 
Here it is worth making an important comment. The proportionality of the fluctuation magnetoconductivity to $h^{2}$ is valid when using the parametrization $\epsilon=\left(T-T_{c 0}\right) / T_{c 0}$ only. As it well known, a weak field shifts the critical temperature linearly, which often makes it attractive to analyze the experimental data by choosing as the reduced temperature parameter $\epsilon_{h}=$ $\left(T-T_{c}(H)\right) / T_{c}(H)$. In this parametrization one can get a term in the magnetoconductivity linear in $h$, which previously was cancelled out by the magnetic field renormalization of the critical temperature. So it is important to recognize that the effect of a weak magnetic field on the fluctuation conductivity cannot be reduced to a simple replacement of $T_{c 0}$ by $T_{c}(H)$ in the appropriate formula without the field. Vice versa, this effect is exactly compensated by the change in the functional dependence of the paraconductivity in magnetic field, and finally it contains the negative quadratic contribution only.

3. Letting the magnetic field go to zero and considering nonzero frequency of the electromagnetic field one can find general expressions for the components of the a.c paraconductivity tensor. They are cumbersome enough and in the complete form can be found, for instance, in [76]. We recall here the simplified asymptotics for the $\operatorname{Re} \sigma$ in the $2 D$ regime only:

$$
\begin{aligned}
\operatorname{Re} \sigma_{(2 D)}^{x x}(r & \ll \epsilon, \widetilde{\omega})= \\
& =\frac{e^{2}}{16 s} \frac{1}{\epsilon}\left[\frac{2 \epsilon}{\widetilde{\omega}} \arctan \frac{\widetilde{\omega}}{\epsilon}-\left(\frac{\epsilon}{\widetilde{\omega}}\right)^{2} \ln \left[1+\left(\frac{\widetilde{\omega}}{\epsilon}\right)^{2}\right]\right] \\
\operatorname{Re} \sigma_{(2 D)}^{z z}(r & \ll \epsilon, \widetilde{\omega})=\frac{e^{2}}{2^{8} s}\left(\frac{s r}{\xi_{x y}}\right)^{2}\left(\frac{1}{\widetilde{\omega}}\right)^{2} \ln \left[1+\left(\frac{\widetilde{\omega}}{\epsilon}\right)^{2}\right] .
\end{aligned}
$$

The general formulas (123)-(124) allow one to study the different crossovers in the a.c. conductivity of layered superconductor in the presence of magnetic field of various intensity. We leave this exercise for the reader having some practical interest in the problem.

\subsection{Magnetic field angular dependence of paraconductivity}

We have seen above that in the case of a geometry with a magnetic field directed along the c-axis many sophisticated fluctuation features of layered superconductors can be studied in the most general form. Nevertheless even the attempt to explore the d.c. conductivity in a longitudinal magnetic field (directed in ab plane) [7] or, moreover, with the field directed at some arbitrary angle $\theta$ with the c-axis leads to the appearance of the a vector potential component in the argument of $\cos \left(k_{z} s\right)$ and the problem requires a nontrivial calculation of the matrix elements over the Mathieu functions.

We already learned that at temperatures very near to the critical one $(\epsilon \ll$ $r$ ) the $3 D$ fluctuation regime takes place. Here the size of the Cooper pairs 
along the c-axis is so large that the peculiarities of the layered structure do not play any more role. This means that only small values of $k_{z}$ are important in the $k_{z}$-integrations, where the $\cos \left(k_{z} s\right)$ in (71) can be expanded and the LD functional is reduced to its traditional GL form with an anisotropic effective mass tensor:

$$
\begin{aligned}
\mathcal{F}[\Psi]= & \int d^{3} \mathbf{r}\left\{a|\Psi|^{2}+\frac{B}{2}|\Psi|^{4}+\sum_{\mu=1}^{3} \frac{1}{4 m_{\mu}}\left|\left(\frac{1}{i} \frac{d}{d x_{\mu}}-2 e A_{\mu}\right) \Psi\right|^{2}+\right. \\
& \left.+\frac{B^{2}}{8 \pi}-\frac{\mathbf{H} \cdot \mathbf{B}}{4 \pi}\right\} .
\end{aligned}
$$

We will demonstrate below that in this case a scaling approach provides a direct access to the most general results by rescaling the anisotropic problem to the corresponding isotropic one on the initial level of the GL approach 78.

Let us suppose that the external field $\mathbf{H}$ is chosen to lie in the $y-z$ plane and makes angle $\theta$ with the $z$ - axis. For sake of simplicity and because the oxide superconductors are within high accuracy uniaxial materials, we choose $m_{x}=m_{y}=m^{*}$, while $m_{z}^{-1}=2 \alpha s^{2} r$ (compare with (69)). The effective anisotropy parameter $\gamma_{a}^{2}=m^{*} / m_{z}=2 \alpha s^{2} r m^{*}<1$ is introduced. In (127) the anisotropy enters only in the gauge-invariant gradient term, so the simple rescaling of the coordinate axes: $x=\widetilde{x}, y=\widetilde{y}, z=\gamma_{a} \widetilde{z}$ together with the scaling of the vector potential: $\mathbf{A}=\left(\widetilde{A}_{x}, \widetilde{A}_{y}, \widetilde{A}_{z} / \gamma_{a}\right)$ will render this term isotropic. The magnetic field evidently is rescaled to $\mathbf{B}=\left(\widetilde{B}_{x} / \gamma_{a}, \widetilde{B}_{y} / \gamma_{a}, \widetilde{B}_{z}\right)$ and the last three terms in (127), describing the magnetic-field energy, are transformed to

$$
\begin{aligned}
\delta \mathcal{F}[\Psi]= & \frac{\gamma_{a}}{8 \pi} \int d^{3} \widetilde{\mathbf{r}}\left[\frac{1}{4 m} \sum_{\mu=1}^{3}\left|\left(\frac{\hbar}{i} \frac{d}{d \widetilde{x}_{\mu}}-\frac{2 e}{c} \widetilde{A}_{\mu}\right) \Psi\right|^{2}+\left(\frac{\widetilde{\mathbf{B}}_{x y}^{2}}{\gamma_{a}}+\widetilde{B}_{z}^{2}\right)-\right. \\
& \left.-2\left(\frac{\widetilde{\mathbf{B}}_{x y} \cdot \mathbf{H}_{x y}}{\gamma_{a}}+\widetilde{B}_{z} H_{z}\right)\right] .
\end{aligned}
$$

In short, we have removed the anisotropy from the gradient term but reintroduced it into the magnetic energy term. In general it is not possible to make both terms isotropic in the Gibbs energy simultaneously. However, depending on the physical question addressed, we can neglect fluctuations in the magnetic field, as was mostly done above.

Let us demonstrate how the method works for the example of the d.c. fluctuation conductivity tensor which was calculated above for a magnetic field directed along the c-axis. We restrict our consideration to the $3 D$ region $(\epsilon \ll r)$. One can write the scaling relations between the electric field and 
current components before and after the scaling transformation by means of a conductivity tensor and the anisotropy parameter:

$$
\begin{array}{rlrl}
j_{x, y} & =\widetilde{j}_{x, y} & j_{z} \sim e v_{z} \sim \gamma_{a} \widetilde{j}_{z} \\
E_{x, y}=\widetilde{E}_{x, y} & E_{z} \sim \frac{\partial \varphi}{\partial z} \sim \frac{1}{\gamma_{a}} \widetilde{E}_{z} .
\end{array}
$$

Now let us rewrite the relations between the current and electric field vectors before and after the scale transformation

$$
\begin{aligned}
& j_{\alpha}=\sigma_{\alpha \beta} E_{\beta} \\
& \widetilde{j}_{\alpha}=\widetilde{\sigma}_{\alpha \beta} \widetilde{E}_{\beta} .
\end{aligned}
$$

Comparing them with $(128)$ and introducing the operator of the direct scaling transformation $T_{\alpha \beta}$

$$
T_{\alpha \beta}=\left(\begin{array}{ccc}
1 & 0 & 0 \\
0 & 1 & 0 \\
0 & 0 & \gamma_{a}
\end{array}\right) \text {, }
$$

one can write $j_{\alpha}=T_{\alpha \mu} \widetilde{j}_{\mu}, E_{\alpha}=\left(T^{-1}\right)_{\alpha \mu} \widetilde{E}_{\mu}$ and express the conductivity tensor as

$$
\sigma_{\alpha \beta}=T_{\alpha \mu} \widetilde{\sigma}_{\mu \rho} T_{\rho \beta}
$$

Now let us work in the already isotropic coordinate frame. We suppose that initially the magnetic field was directed along the c-axis and now we rotate it in the $\mathrm{X}-\mathrm{Z}$ plane by the angle $\widetilde{\theta}$ with respect to the initial direction. The conductivity tensor will be transformed by the usual matrix law:

$$
\widetilde{\sigma}_{\alpha \beta}(\widetilde{\theta})=R_{\alpha \mu}^{T} \widetilde{\sigma}_{\mu \rho}(0) R_{\rho \beta}=R_{\alpha \mu}^{T}\left(T^{-1}\right)_{\mu \varsigma} \sigma_{\varsigma \eta}(0)\left(T^{-1}\right)_{\eta \delta} R_{\delta \beta}
$$

and

$$
\sigma_{\alpha \beta}(\widetilde{\theta})=T_{\alpha \gamma} \widetilde{\sigma}_{\gamma \delta}(\widetilde{\theta}) T_{\delta \beta}=T_{\alpha \gamma} R_{\gamma \mu}^{T}\left(T^{-1}\right)_{\mu \varsigma} \sigma_{\varsigma \eta}(0)\left(T^{-1}\right)_{\eta \delta} R_{\delta \kappa} T_{\kappa \beta},
$$

where

$$
R_{\alpha \beta}=\left(\begin{array}{ccc}
\cos \tilde{\theta} & 0 & -\sin \widetilde{\theta} \\
0 & 1 & 0 \\
\sin \tilde{\theta} & 0 & \cos \widetilde{\theta}
\end{array}\right) .
$$

Finally the fluctuation conductivity tensor $\sigma_{\alpha \beta}(\theta)$ in the initial tetragonal system with the magnetic field directed at the angle $\theta$ with respect to the 
c-axis can be expressed by means of the effective transformation operator $M_{\alpha \beta}$ :

$$
\sigma_{\alpha \beta}(\theta)=M_{\alpha \varsigma}^{T}(\widetilde{\theta}) \sigma_{\varsigma \eta}(0, \widetilde{H}) M_{\eta \beta}(\widetilde{\theta}),
$$

with

$$
M_{\alpha \beta}(\widetilde{\theta})=\left(T^{-1}\right)_{\alpha \delta} R_{\delta \kappa} T_{\kappa \beta}=\left(\begin{array}{ccc}
\cos \widetilde{\theta} & 0 & -\frac{1}{\gamma} \sin \widetilde{\theta} \\
0 & 1 & 0 \\
\gamma_{a} \sin \widetilde{\theta} & 0 & \cos \widetilde{\theta}
\end{array}\right) .
$$

The angle $\widetilde{\theta}$ can be expressed via the renormalized magnitude of the magnetic field $\widetilde{H}=\sqrt{H_{c}^{2}+\gamma_{a}^{2} H_{x}^{2}}$ :

$$
\cos \widetilde{\theta}=\frac{\widetilde{H}_{z}}{\widetilde{H}}=\frac{\cos \theta}{\sqrt{\cos ^{2} \theta+\gamma_{a}^{2} \sin ^{2} \theta}} ; \sin \tilde{\theta}=\frac{\gamma_{a} \sin \theta}{\sqrt{\cos ^{2} \theta+\gamma_{a}^{2} \sin ^{2} \theta}} .
$$

In the case of the paraconductivity of a layered superconductor with the magnetic field applied at an arbitrary angle $\theta$ the answer can be written in the general form by means of the three diagonal components of conductivity $\sigma_{i i}(0, \widetilde{H})$ in the perpendicular field $\widetilde{H}$ :

$$
\begin{aligned}
\sigma_{\alpha \beta}(\theta)= & \frac{1}{\cos ^{2} \theta+\gamma_{a}^{2} \sin ^{2} \theta} \times \\
& \times\left(\begin{array}{ccc}
\sigma_{x x} \cos \theta+ & 0 & \sigma_{z z}^{2} \gamma_{a} \sin ^{2} \theta \cos \theta- \\
+\gamma_{a}^{4} \sigma_{z z} \sin ^{2} \theta & & -\sigma_{x x} \sin \theta \\
0 & \sigma_{y y}\left(\cos ^{2} \theta+\gamma_{a}^{2} \sin ^{2} \theta\right) & 0 \\
\sigma_{z z}^{2} \gamma_{a} \sin \theta \cos \theta & 0 & \sigma_{z z} \cos ^{2} \theta
\end{array}\right)
\end{aligned}
$$

In the simplest case of a longitudinal field $\theta=90^{\circ}$ :

$$
\sigma_{\alpha \beta}\left(90^{0}, H\right)=\left(\begin{array}{ccc}
\gamma_{a}^{2} \sigma_{z z}\left(0, \gamma_{a} H\right) & 0 & -\gamma_{a}^{-2} \sigma_{z z}\left(0, \gamma_{a} H\right) \\
0 & \sigma_{y y}\left(0, \gamma_{a} H\right) & 0 \\
0 & 0 & 0
\end{array}\right)
$$

\section{Fluctuations near superconductor-insulator transition}

\subsection{Quantum phase transition}

It is usually supposed that the temperature of the superconducting transition does not depend on the concentration of non-magnetic impurities (Anderson's theorem [79,80]). Nevertheless when the degree of disorder is very 
high Anderson localization takes place, and it would be difficult to expect that under conditions of strong electron localization superconductivity can exist, even if there is inter-electron attraction. This means that at $T=0$ the phase transition takes place with a change of the disorder strength or carrier concentration. Such a transition is called a quantum phase transition since at zero temperature the classical fluctuations are absent. Indeed, one can see from (7) that in the limit $T \rightarrow 0$ the thermal fluctuation Cooper pairs vanish.

In the metallic phase of a disordered system the conductivity is mostly determined by the weakly decaying fermionic excitations, their dynamics yielding the familiar Drude formula (the method which accounts for the fermionic excitations will be referred to as the Fermi approach later on). Inside the critical region the charge transfer due to fluctuation Cooper pairs turns out to be more important. In some approximation, the pairs may be considered as Bose particles. Therefore the approach dealing with the fluctuation pairs will be called below the Bose approach.

Let us suppose that at temperature $T=0$ the superconducting state occurs in a weakly disordered system. In principle two scenarios of the development of the situation are possible with an increase of the disorder strength: the system at some critical disorder strength can go from the superconducting state to the metallic state or to the insulating state. The first scenario is natural and takes place in the following cases: if the effective constant of the inter-electron interaction changes its sign with the growth of the disorder; if the effective concentration of magnetic impurities increases together with the disorder growth; if the pairing symmetry of superconducting state is nontrivial it can be destroyed even by the weak disorder level. We will study here the second scenario where the superconductor becomes an insulator with disorder increase. This means that at some disorder degree range, higher than the localization edge when the normal phase does not exist any more at finite temperatures, superconductivity can still survive. From the first glance this statement seems strange: what does superconductivity mean if the electrons are already localized? And if it really can take place beyond the metallic phase, at what value of disorder strength and in which way does the superconductivity finally disappears?

One has to have in mind that localization is a quantum phenomenon in its nature and with the approach to the localization edge the coherence length of localization $\ell$ grows. From the insulator side of the transition vicinity this means the existence of large scale regions where delocalized electrons exist. If the energy level spacing in such regions does not exceed the value of superconducting gap Cooper pairs still can be formed by the delocalized electrons of this region.

The problem can be reformulated in other, already familiar, way: how does the critical temperature of the superconducting transition decrease with the increase of the disorder strength? In the previous Sections we have already tried to solve it by discussing the critical temperature fluctuation shift. We 
have seen that the fluctuation shift of the critical temperature is proportional to $\sqrt{G i_{(3)}}$ for a $3 D$ superconductor and to $G i_{(2)} \ln \left\{1 / G i_{(2)}\right\}$ for $2 D$. This means that the critical temperature is not changed noticeably as long as the Ginzburg-Levanyuk number remains small. So one can expect the complete suppression of superconductivity when $G i \sim 1$ only. For further consideration it is convenient to separate the $3 D$ and $2 D$ cases because the physical pictures of the superconductor-insulator transition for them are quite different.

\subsection{D superconductors}

As one can see from Table 1 in the $3 D$ case the Ginzburg-Levanyuk number remains small at $p_{F} l \sim 1: G i_{(3 d)} \approx\left(\frac{T_{c}}{E_{F}}\right) \ll 1$. Nevertheless, approaching the edge of localization, the width of the fluctuation region increases [81. In the framework of the self-consistent theory of localization 82 such growth of the width of the fluctuation region was found in paper [83].

Instead of the cited self-consistent theory let us make some more general assumptions concerning the character of the metal-insulator (M-I) transition in the absence of superconductivity [13]. We suppose that in the case of very strong disorder and not very strong Coulomb interaction the M-I transition is of second order. The role of "temperature" for this transition is played by the "disorder strength" which is characterized by the dimensionless value $g=\frac{p_{F} l}{2 \pi}$ With its decrease the conductivity of the metallic phase decreases and at some critical value $g_{c}$ tends to zero as

$$
\sigma=e^{2} p_{F}\left(g-g_{c}\right)^{\varkappa}
$$

This is the critical point of the Anderson (M-I) transition. We assume that the thermodynamic density of states remains constant at the transition point.

The electron motion in metallic phase far enough from the M-I transition has a diffusion character and the conductivity can be related to the diffusion coefficient $\mathcal{D}=p_{F} l / 3 m$ by the Einstein relation: $\sigma=\nu e^{2} \mathcal{D}$. One can say that diffusion like "excitations" with the spectrum $\omega(q)=i D q^{2}$ propagate in the system. At the point of the M-I transition normal diffusion terminates and conductivity, together with $\mathcal{D}$, turns zero. In accordance with scaling ideas, the diffusion coefficient can be assumed here to be a power function of $q$ : $\mathcal{D}(q) \sim q^{z-2}$, with the dynamical critical exponent $z>2$. The anomalous diffusion excitation spectrum in this case would take the form $\omega \sim q^{z}$.

In the insulating phase $\left(g<g_{c}\right)$ some local, anomalous diffusion, confined to regions of the scale $\ell$, is still possible. It cannot provide charge transfer through out all the system, so $\mathcal{D}(q=0)=0$, but for small distances $\left(q \gtrsim \ell^{-1}\right)$ anomalous diffusion takes place. Analogously, in the metallic phase $\left(g>g_{c}\right)$ the diffusion coefficient in the vicinity of the transition has an anomalous dependence on $q$ for $q \gtrsim \ell^{-1}$ and weakly depends on it for $q \lesssim \ell^{-1}$. So one can conclude that the diffusion coefficient for $q \gtrsim \ell^{-1}$ from both sides of the 
transition has the same $q$-dependence as for all $q$ in the transition point. It can be written in the form

$$
\mathcal{D}(q)=\frac{g}{3 m}\left[\frac{\varphi(q \ell)}{p_{F} \ell}\right]^{z-2}, \varphi(x)=\left\{\begin{array}{l}
x, x \gg 1 \\
1, x \ll 1, g>g_{c} \\
0, x \ll 1, g<g_{c}
\end{array},\right.
$$

where the dimensionless localization length $\ell$, characterizing the spatial scale near the transition, grows with the approach to the transition point like

$$
\ell(g)=\frac{1}{p_{F}}\left(g-g_{c}\right)^{-\frac{\varkappa}{z-2}} .
$$

The critical exponent in this formula is found from the Einstein relation in the vicinity of the M-I transition.

At finite temperatures, instead of the critical point $g_{c}$, a crossover from metallic to insulating behavior of $\sigma(g)$ takes place. The width of the crossover region is $\widetilde{g}-g_{c}$, where $\widetilde{g}$ is determined from the relation $\mathcal{D} \ell^{-2}(\widetilde{g}) \sim E_{F}\left[p_{F} \ell(\widetilde{g})\right]^{-z} \sim$ $T$ (we have used the second asymptotic of (131)). In this region the diffusion coefficient is

$$
\mathcal{D}(T) \sim T \ell^{2} \sim \frac{T}{p_{F}^{2}}\left(\frac{E_{F}}{T}\right)^{\frac{2}{z}}
$$

and it depends weakly on the $g-g_{c}$. Beyond this region the picture of the transition remains the same as at $T=0$.

Let us consider now what happens to superconductivity in the vicinity of the localization transition. In the mean field approximation (BCS) the thermodynamic properties of a superconductor do not depend on the character of the diffusion of excitations. This should be contrasted with the fluctuation theory, where such a dependence clearly exists. We will show that the type of superconducting transition depends on the dynamical exponent $z$. If $z>3$, the transition to superconductivity occurs on the metallic side of the localization transition (we will refer to such a transition as S-N transition). If $z<3$, the transition to superconductivity occurs from the insulating state directly (S-I transition).

Let us study how the superconducting fluctuations affect the transition under discussion. In spirit of the GL approach fluctuation phenomena in the vicinity of the transition can be described in the framework of the GL functional (26). The coherence length in the metallic region, far enough from the Anderson transition, was reported in Introduction to be equal $\xi^{2}=\xi_{c} l=0.42 \mathcal{D} / T$. In the vicinity of the M-I transition we still believe in the diffusive character of the electron motion resulting in the pair formation. The only difference from the previous consideration is the anomalous character of the quasiparticle diffusion. So in order to describe the superconducting fluctuations simultaneously near superconducting (in temperature) 
and Anderson (in $g$ ) transitions let us use the GL functional (26) with the $k$-dependent diffusion coefficient (131).

The value of $G i$ can be estimated from the expression for the fluctuation contribution to heat capacity (30) taken at $\epsilon \sim G i$, where the fluctuation correction reaches the value of the heat capacity jump:

$$
1 \sim \frac{T}{\nu} \int \frac{d^{3} q}{\left(T G i+\mathcal{D}(q) q^{2}\right)^{2}}
$$

with $T \simeq T_{c}$. Let us approach the M-I transition from the metallic side. If we are far enough from transition, $G i$ is small and the integral in (134) is determined by the region of small momenta $\mathcal{D}(q) q^{2} \lesssim T G i$ :

$$
G i \sim \frac{T}{\nu^{2} \mathcal{D}^{3}(q=0)} .
$$

Two scenarios are possible: Gi becomes of the order of 1 in the metallic phase, or it remains small up to the crossover region, where finally reaches its saturation value. In the first case we can use the second asymptotic of (131) for $D(q)$ and find:

$$
G i \sim \frac{T_{c}}{E_{F}}\left(p_{F} \ell\right)^{3 z-6} \text {. }
$$

One can see that $G i$ becomes of the order of 1 at $p_{F} \ell_{M} \sim\left(E_{F} / T\right)^{\frac{1}{3 z-6}}$. Comparing this value with $p_{F} \ell(\widetilde{g}) \sim\left(E_{F} / T\right)^{1 / z}$ at the limit of the crossover region we see that for $z>3$ the first scenario is realized. Concluding the first scenario discussion we see that the superconducting critical temperature goes to zero at $\ell=\ell_{M}$, still in the metallic phase, so at $T=0$ a superconductornormal phase (S-N) type quantum phase transition takes place.

The second scenario takes place for $z<3$ when Gi remains small even at the edge of crossover region, reaching there the value

$$
G i \sim\left(\frac{T_{c}}{E_{F}}\right)^{\frac{2(3-z)}{z}} \ll 1 .
$$

In the crossover region the diffusion coefficient, and hence $G i$, almost do not vary. This is why the temperature of superconducting transition remains almost frozen with further increase of disorder driving the system through the Anderson transition. The abrupt growth of $G i$ and decrease of $T_{c}$ take place when the system finally goes from the crossover to the insulating region. In the insulator phase the diffusion coefficient $D\left(q \lesssim l^{-1}\right)=0$ and from (134) one can find for $G i$ :

$$
G i \sim \sqrt{\frac{E_{F}}{T_{c}}} \frac{1}{\left(p_{F} \ell\right)^{3 / 2}}
$$


Comparing this result with the Table 1 it is easy to see that it coincides with the Ginzburg-Levanyuk number for a zero-dimensional granule of size $\ell \ll \xi(T)$. Hence we see that in the second scenario the Ginzburg number reaches 1 and, respectively, $T_{c} \rightarrow 0$ at $p_{F} \ell_{I} \sim\left(\frac{E_{F}}{T_{c}}\right)^{1 / 3}$, which is far enough from the M-I transition point. This is why in this case one can speak about the realization at $T=0$ of a superconductor-insulator (S-I) type quantum phase transition. The scale $\ell_{I}$ determines the size of the "conducting" domains in the insulating phase, where the level spacing reaches the order of the superconducting gap. It is evident that in the domain of scale $\ell \lesssim \ell_{I}$ superconductivity cannot be realized.

In the vicinity of a quantum phase transition one can expect the appearance of non monotonic dependencies of the resistance on temperature and magnetic field. Indeed, starting from the zero resistance superconducting phase and increasing temperature from $T=0$, the system passes through the localization region, where the resistance is high, to high temperatures where some hopping charge transfer will decrease the resistance again. The analogous speculations are applicable to the magnetic field effect: first the magnetic field "kills" superconductivity and increases the resistance, then it destroys localization and decreases it.

The phase diagram in the $(T, g)$ plane has the form sketched in Fig. 5 . For $g_{I}=g_{c}-\left(T_{c} / E_{F}\right)^{3(z-2) / \varkappa}$, an S-I transition takes place at $T=0$. Increasing the temperature from $T=0$ in the region $0 \lesssim g \lesssim g_{I}$ we remain in the insulating phase with exponential dependence of resistance on temperature. For $g_{I} \lesssim g \lesssim \widetilde{g}_{-}$at low temperatures $0 \leq T<T_{c}(g)$ the system stays in the superconducting state which goes to the insulating phase at higher temperatures. In the vicinity of the Anderson transition $\left(\widetilde{g}_{-} \lesssim g \lesssim \widetilde{g}_{+}\right)$the superconducting state goes with growth of the temperature to some crossover metal-insulator state which is characterized by a power decrease of the resistivity with the increase of temperature. Finally at $g_{c} \lesssim g$ the superconducting phase becomes of the BCS type and at $T=T_{c}$ it goes to a metallic phase.

The phase diagram in the magnetic field - disorder plane is similar to that in the $(T, g)$ plane with the only difference that at $T=0$ there is no crossover region, instead a phase transition takes place.

\subsection{D superconductors}

Preliminaries. As was demonstrated in Section 4, according to the conventional theory of paraconductivity, the sheet conductivity in the vicinity of the superconducting transition is given by a sum of the electron residual conductivity $g e^{2}$ (Fermi part) and the conductivity of the Cooper pair fluctuations (Bose part) (see (101)). This expression is valid in the Ginzburg Landau region when the second term is a small correction to the first one. 

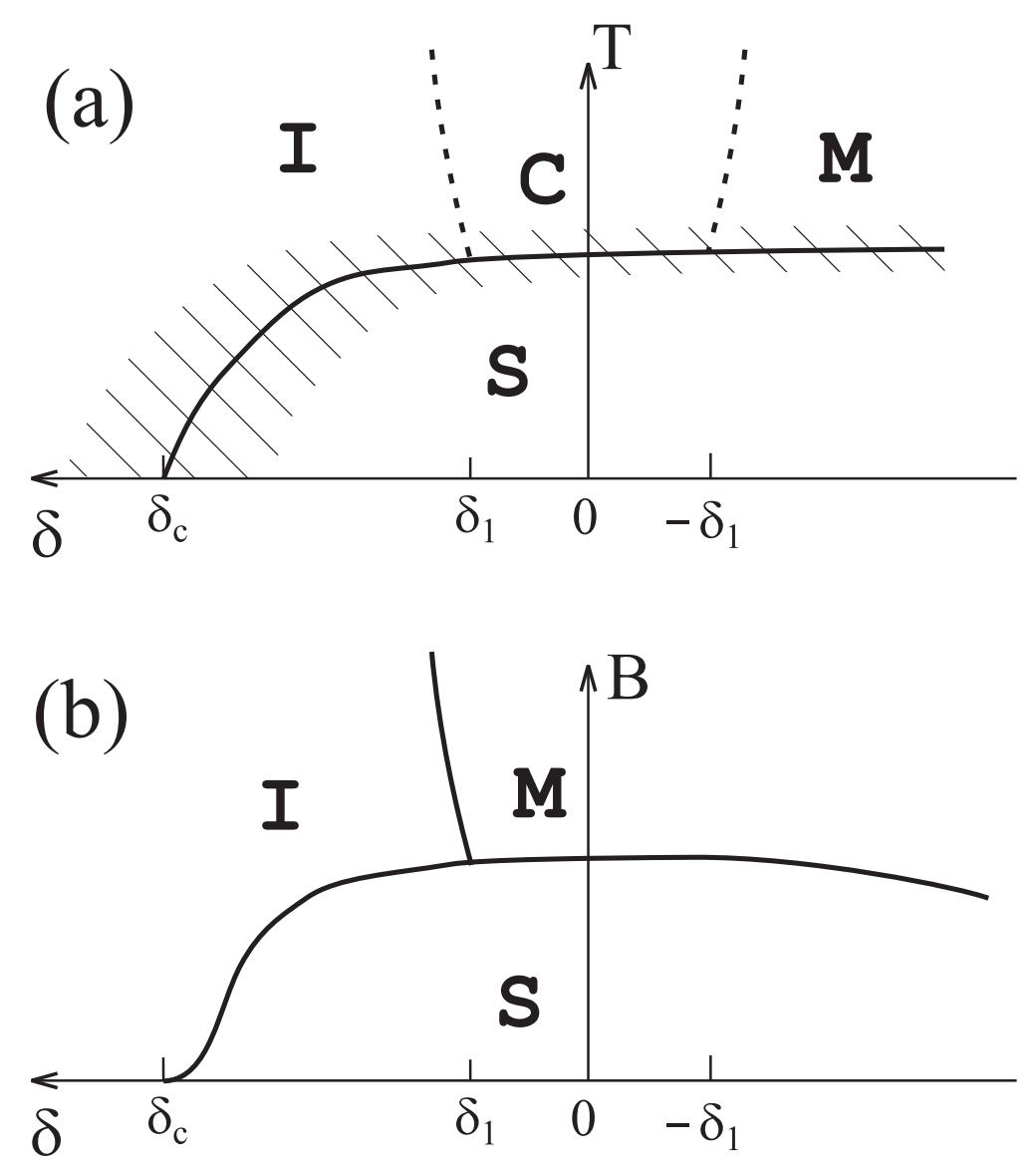

Fig. 5. Phase diagram in the temperature - disorder plane for a three-dimensional superconductor.

The width of the critical region can be determined from the requirement of equality of both contributions in (101) ${ }^{18}$ :

$$
\epsilon_{c r}=\frac{1}{16 g}=1.3 G i_{(2 d)} .
$$

In accordance with general scaling ideas one can believe that inside the fluctuation region the conductivity should obey the form:

$$
\sigma(T)=g e^{2} f\left(\frac{\epsilon}{G i_{(2 d)}}\right) .
$$

${ }^{18}$ It is worth mentioning that this definition of the Ginzburg Levanyuk number $\left(\widetilde{G i} i_{(2 d)}=\frac{\pi}{8 p_{F} l}\right)$ agrees with that defined from the heat capacity fluctuations $\left(G i_{(2 d)}=\frac{0.3}{p_{F} l}\right)$. 
Concerning the scaling function $f(x)$, we know its asymptotes in the mean field region $(x \gg 1)$ and just above the BKT transition 56.57:

$$
f(x)=\left\{\begin{array}{ll}
1+x^{-1}, & x \gg 1 \\
\left.\exp \left[-b\left(x-x_{\mathrm{BKT}}\right)^{-1 / 2}\right)\right], & x \rightarrow x_{\mathrm{BKT}}=-4
\end{array} .\right.
$$

The BKT transition temperature $T_{c}^{\mathrm{BKT}}$ is determined by

$$
n_{s 2}\left(T_{c}\right)=\frac{4 m T_{c}}{\pi} .
$$

and one can find its value by comparing the superfluid density $n_{s}$ from (142) with that found in the BCS scheme:

$$
T_{c}^{\mathrm{BKT}}=T_{c 0}(1-4 G i) .
$$

Here we assumed that the Ginzburg parameter is small, so that the BKT transition temperature does not deviate much from the mean-field BCS transition temperature $T_{c 0}$.

Boson mechanism of the $\boldsymbol{T}_{\boldsymbol{c}}$ suppression. The classical and quantum fluctuations reduce $n_{s}$ and therefore, suppress $T_{c}^{\mathrm{BKT}}$. At some $g=g_{c} \sim 1$, the superfluid density $n_{s}$, and simultaneously $T_{c}^{\mathrm{BKT}}$, go to zero. In the vicinity of this critical concentration of impurities $T_{c}^{\mathrm{BKT}} \ll T_{c 0}$. Thus a wide new window of intermediate temperatures $T_{c}^{\mathrm{BKT}} \ll T \ll T_{c 0}$ opens up. In this window, according the dynamical quantum scaling conjecture [84, one finds

$$
\sigma=e^{2} \varphi\left(\frac{T}{T_{c}^{B K T}}\right)
$$

At $T-T_{c}^{\mathrm{BKT}} \ll T_{c}^{\mathrm{BKT}}$ the Berezinski-Kosterlitz-Thouless law (140)-(141) should hold, so $\varphi(x)=f(x)$ and is exponentially small. In the intermediate region $T_{c}^{\mathrm{BKT}} \ll T \ll T_{c}^{\mathrm{BCS}}$ the duality hypothesis gives $\varphi(x)=\pi / 2$. Let us derive this relation.

We will start from the assumption that in the region $T_{c}^{\mathrm{BKT}} \ll T \ll T_{c 0}$ the conductivity is a universal function of temperature which does not depend on the pair interaction type. Being in the framework of the classical approach, let us suppose that in a weak electric field pairs move with the velocity $\mathbf{v}=\mathbf{F} / \eta$, where $\mathbf{F}=2 e \mathbf{E}$ is the force acting on the pairs. The current density $\mathbf{j}=2 e n \mathbf{v}=\sigma \mathbf{E}$, (here $n$ is the pair density), so one can relate the conductivity with the effective viscosity $\eta: \sigma=4 e^{2} n / \eta$.

Let us recall that we are dealing with a quantum fluid, so another, superconducting, view on the problem of its motion near the quantum phase transition exists. One can say that with the increase of $G i$ the role of quantum fluctuations grows too and fluctuation vortices carrying the magnetic flux quantum $\Phi_{0}=\pi / e$ are generated. With electric current flow in the system the Lorentz (Magnus) force acts on a vortex: $F=j \Phi_{0}$. The electric field 
is equal to the rate of magnetic flux transfer, i.e. to the density of the vortex current: $\mathbf{E}=\Phi_{0} n_{v} \mathbf{v}_{v}=\Phi_{0} n_{v} \mathbf{F} / \eta_{\mathbf{v}}$, where $n_{v}$ is the density and $\eta_{v}$ is the viscosity of the vortex liquid. As a result $\mathbf{E}=\Phi_{0}^{2} n_{v} \mathbf{j} / \eta_{\mathbf{v}}=\mathbf{j} / \sigma$. So one can conclude that for vortices the velocity is proportional to the voltage, and the force is proportional to the current. For Cooper pairs (bosons) the situation is exactly the opposite.

The duality hypothesis consists in the assumption that at the critical point the pair and the vortex liquid density flows are equal: $n_{v} \mathbf{v}_{v}=n \mathbf{v}$. Comparing these quantities, expressed in terms of the conductivity from the above relations, one can find a universal value for the conductivity at the critical point

$$
\sigma=\frac{2 e}{\Phi_{0}}=\frac{2 e^{2}}{\pi} .
$$

One can restrict oneself to a less strong duality hypothesis, supposing the product $n \eta=C T^{\delta}$ with a universal $\delta$ exponent both for the pair and the vortex liquids, while the constant $C$ for them is different. In this case, based on duality, is possible to demonstrate that $\delta=0$ and the conductivity is temperature independent up to $T_{c 0}$ but its value is not universal any more and can vary from one sample to another.

To conclude, let us emphasize that in the framework of the boson scenario of superconductivity suppression, the BCS critical temperature is changed insignificantly, while the "real" superconducting transition temperature $T_{c}^{\mathrm{BKT}} \rightarrow$ 0 .

Fermion mechanism of $\boldsymbol{T}_{\boldsymbol{c}}$ suppression. Apart from the above fluctuation (boson) mechanism of the suppression of the critical temperature in the $2 D$ case, there exists another, fermionic mechanism. The suppressed electron diffusion results in a poor dynamical screening of the Coulomb repulsion which, in turn, leads to the renormalization of the inter-electron interaction in the Cooper channel. and hence to the dependence of the critical temperature on the value of the high-temperature sheet resistivity of the film. As long as the correction to the non-renormalized BCS transition temperature $T_{c 0}$ is still small, one finds $85,86,87$ :

$$
T_{c}=T_{c 0}\left(1-\frac{1}{12 \pi^{2} g} \ln ^{3} \frac{1}{T_{c 0} \tau}\right) .
$$

At small enough $T_{c 0}$ this mechanism of critical temperature suppression turns out to be the principal one. The suppression of $T_{c}$ down to zero in this case may happen in principle even at $g \gg 1$. A renormalization group analysis gives [88] the corresponding critical value of conductance

$$
g_{c}=\left(\frac{1}{2 \pi} \ln \frac{1}{T_{c 0} \tau}\right)^{2} .
$$


Here we should recall that the typical experimental [89] values of $g_{c}$ are in the region $g_{c} \sim 1-2$, and do not differ dramatically from the predictions of the boson duality assumption $g_{c}=\frac{2}{\pi}$. If one attempts to explain the suppression of $T_{c}$ within the fermion mechanism, one should assume that $\ln \frac{1}{T_{c 0} \tau}>5$. Then, according to Eq. (147), $g_{c}>2 / \pi$ and the boson mechanism is not important. On the contrary, if $\ln \frac{1}{T_{c 0} \tau}<4$, then Eq. (146) gives a small correction for $T_{c}$ even for $g_{c}=2 / \pi$ and the fermion mechanism becomes unimportant. The smallness of the critical temperature $T_{c}$ compared to the Fermi energy is the cornerstone of the BCS theory of superconductivity and it is apparently satisfied even in high- $T_{c}$ materials. Nevertheless it is necessary to use the theoretically large logarithmic parameter with care, if one needs $\ln \frac{1}{T_{c 0} \tau}$ to be as large as 4 .

\section{Microscopic derivation of the Time-Dependent Ginzburg-Landau equation}

\subsection{Preliminaries}

We have seen above how the phenomenological approach based on the GL functional allows one to describe fluctuation Cooper pairs (Bose particles) near the superconducting transition and to account for their contribution to different thermodynamical and transport characteristics of the system. Now we pass to the discussion of the microscopic description of fluctuation phenomena in superconductors. The development of the microscopic approach is necessary for the following reasons:

1. This description permits microscopic determination of the values of the phenomenological parameters of the GL theory.

2. This method is more powerful than the phenomenological GL approach and permits treatment of fluctuation effects quantitatively even far from the transition point and for magnetic fields strong as $H_{c 2}$, taking into account the contributions of dynamical and short wavelength fluctuations.

3 . The electron energy relaxation times in metals are relatively large $\left(\tau_{\varepsilon} \gg\right.$ $\hbar / T$ ) which causes the electron low frequency dynamics to be sensitive to the nearness to the superconducting transition. This is why the temperature dependence of fluctuation corrections can be determined generally speaking not only by the Cooper pair motion but also by changes in the single-electron properties.

4. There are some fluctuation phenomena in which the direct Cooper pair contribution is considerably suppressed or even absent altogether. Among them we can mention the nuclear magnetic relaxation rate, tunnel conductivity, c-axis transport in strongly anisotropic layered metals, thermoelectric power and heat conductivity where the fluctuation pairing manifests itself by means of the indirect influence on the properties of the single-particle states of electron system. 
Formally in the above consideration averaging over the superconducting order parameter has been accomplished by means of a functional integration over all its possible bosonic field configurations. In this description we have dealt with the fluctuation Cooper pair related effects only and the method of the functional integration turned out to be simple and effective for their description. In the following Sections we will develop the diagrammatic method of Matsubara temperature Green functions which is more adequate for the description of the properties of a Fermi system of interacting electrons.

\subsection{The Cooper channel of electron-electron interaction}

Let us start the microscopic description of fluctuation phenomena in a superconductor from the electron Hamiltonian. We will choose it in the simple BCS form 19 :

$$
\mathcal{H}=\sum_{\mathbf{p}, \sigma} E(\mathbf{p}) \widetilde{\psi}_{\mathbf{p}, \sigma}^{+} \widetilde{\psi}_{\mathbf{p}, \sigma}+g \sum_{\mathbf{p}, \mathbf{p}^{\prime}, \mathbf{q}, \sigma, \sigma^{\prime}} \widetilde{\psi}_{\mathbf{p}+\mathbf{q}, \sigma}^{+} \widetilde{\psi}_{-\mathbf{p},-\sigma}^{+} \widetilde{\psi}_{-\mathbf{p}^{\prime},-\sigma^{\prime}} \widetilde{\psi}_{\mathbf{p}^{\prime}+\mathbf{q}, \sigma^{\prime}} \cdot(148
$$

The momentum conservation law side by side with singlet pairing are already taken into account in the interaction term. Here $E(\mathbf{p})$ is the quasiparticle spectrum of the normal metal; $g$ is the negative constant of electron-electron attraction which is supposed to be momentum independent and different from zero in a narrow domain of momentum space in the vicinity of the Fermi surface where

$$
p_{F}-\frac{\omega_{D}}{v_{F}}<|\mathbf{p}|,\left|\mathbf{p}^{\prime}\right|<p_{F}+\frac{\omega_{D}}{v_{F}} .
$$

$\widetilde{\psi}_{\mathbf{p}, \sigma}^{+}$and $\widetilde{\psi}_{\mathbf{p}, \sigma}$ are the creation and annihilation field operators in the Heisenberg representation, so the first term is just the kinetic energy of the noninteracting Fermi gas. The interaction term is chosen in the traditional form characteristic for the electron-phonon mechanism of superconductivity20.

For the description of the properties of an interacting electron system with the Hamiltonian (148) we will use the formalism of the Matsubara tempera-

${ }^{19}$ We suppose that reader is familiar with the BCS formulation of the theory of superconductivity (see for example, [92]).

${ }^{20}$ Fluctuations in the framework of more realistic Eliashberg 90 model of superconductivity were studied by B.Narozhny 91. He demonstrated that the strong coupling does not change drastically the results of the weak coupling approximation. The critical exponents turn out to be exactly the same as in the framework of the GL theory, which provides an adequate description of paraconductivity in strong coupling superconductors. The robustness of the critical exponents and their dependence in GL region on the space dimensionallity only was stressed in 83 in relation to the discussion of the paraconductivity at the edge of the superconductor-insulator transition. 
ture diagrammatic technique. The state of a non-interacting quasiparticle is described by its Green function

$$
G\left(\mathbf{p}, \varepsilon_{n}\right)=\frac{1}{i \varepsilon_{n}-\xi(\mathbf{p})},
$$

where $\varepsilon_{n}=(2 n+1) \pi T$ is a fermion Matsubara frequency and $\xi(\mathbf{p})=$ $E(\mathbf{p})-E_{F}$ is the quasiparticle energy measured from the Fermi level.

As it is well known the effective electron-electron attraction leads to a reconstruction of the ground state of the electron system which formally manifests itself by the appearance at the critical temperature of a pole in the two particle Green function

$$
\mathcal{L}\left(p, p^{\prime}, q\right)=<T_{\tau}\left[\widetilde{\psi}_{p+q, \sigma} \widetilde{\psi}_{-p,-\sigma} \widetilde{\psi}_{p^{\prime}+q, \sigma^{\prime}}^{+} \widetilde{\psi}_{-p^{\prime},-\sigma^{\prime}}^{+}\right]>,
$$

where $T_{\tau}$ is the time ordering operator and $4 D$ vector notations are used 92 . As it well known the two particle Green function can be expressed in terms of the vertex part 92. In the case under consideration it is the vertex part of the electron-electron interaction in the Cooper channel $L\left(\mathbf{q}, \Omega_{k}\right)$, which will be called below the fluctuation propagator. The Dyson equation for $L\left(\mathbf{q}, \Omega_{k}\right)$, accounting for the e-e attraction in the ladder approximation, is represented graphically in Fig.6. It can be written down analytically as

$$
L^{-1}\left(\mathbf{q}, \Omega_{k}\right)=g^{-1}-\Pi\left(\mathbf{q}, \Omega_{k}\right),
$$

where the polarization operator $\Pi\left(\mathbf{q}, \Omega_{k}\right)$ is defined as a loop of two singleparticle Green functions:

$$
\Pi\left(\mathbf{q}, \Omega_{k}\right)=T \sum_{\varepsilon_{n}} \int \frac{d^{3} \mathbf{p}}{(2 \pi)^{3}} G\left(\mathbf{p}+\mathbf{q}, \varepsilon_{n+k}\right) G\left(-\mathbf{p}, \varepsilon_{-n}\right) .
$$

Let us emphasize, that the two quantities introduced above, $\mathcal{L}\left(p, p^{\prime}, q\right)$ and $L(q)$, are closely connected with each other. The former being integrated over momenta $p$ and $p^{\prime}$ becomes an average of the product of two order parameters:

$$
\int d p d p^{\prime} \mathcal{L}\left(p, p^{\prime}, q\right)=\frac{1}{g^{2}}\left\langle\Delta_{q} \Delta_{q}^{*}\right\rangle,
$$

where $\Delta_{q}$ is the superconducting gap proportional to the condensate wave function $\Psi$. Thus, this quantity represents the coefficient in the linear term in the GL equation. In terms of the polarization operator introduced above it can be written as

$$
\int d p d p^{\prime} \mathcal{L}\left(p, p^{\prime}, q\right) \propto \frac{\Pi}{1-g \Pi} .
$$




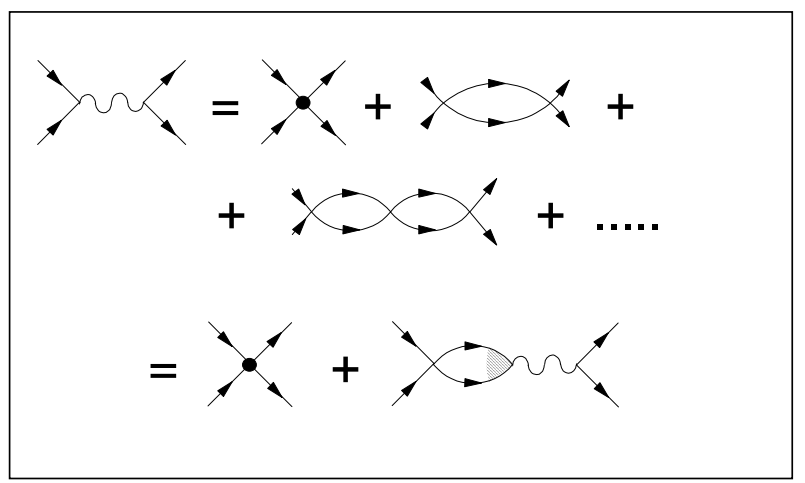

Fig. 6. The Dyson equation for the fluctuation propagator (wavy line) in the ladder approximation. Solid lines represent one-electron Green functions, bold points correspond to the model electron-electron interaction.

Comparing this equation with Eq. $(150)$ for the fluctuation propagator we see that the corresponding expressions are very similar. After analytical continuation to the real frequencies the fluctuation propagator $L(q, i \Omega)$ coincides with the quantity defined by Eq.(152) (up to a constant).

One can calculate the propagator (150) using the one-electron Green functions of the normal metal (149). For sake of convenience of future calculations let us define the correlator of two one-electron Green functions

$$
\begin{aligned}
\mathcal{P}\left(\mathbf{q}, \varepsilon_{1}, \varepsilon_{2}\right) & =\int \frac{d^{3} \mathbf{p}}{(2 \pi)^{3}} G\left(\mathbf{p}+\mathbf{q}, \varepsilon_{1}\right) G\left(-\mathbf{p}, \varepsilon_{2}\right)= \\
& =2 \pi \nu \Theta\left(-\varepsilon_{1} \varepsilon_{2}\right)\left\langle\frac{1}{\left|\varepsilon_{1}-\varepsilon_{2}\right|+\left.i \Delta \xi(\mathbf{q}, \mathbf{p})\right|_{\epsilon(\mathbf{p})=E_{F}}}\right\rangle_{F . S .},
\end{aligned}
$$

where $\Theta\left(-\varepsilon_{1} \varepsilon_{2}\right)$ is Heavyside step function, $\nu$ is the one-electron density of states, $<>_{F . S .}=\int \frac{d \Omega_{\mathrm{p}}}{4 \pi}$ means the averaging over the Fermi surface,

$$
\left.\Delta \xi(\mathbf{q}, \mathbf{p})\right|_{\epsilon(\mathbf{p})=E_{F}}=\left.[\xi(\mathbf{q}+\mathbf{p})-\xi(-\mathbf{p})]\right|_{\epsilon(\mathbf{p})=E_{F}} \approx\left(\mathbf{v}_{\mathbf{p}} \mathbf{q}\right)_{\xi(\mathbf{p})=0} .
$$

The last approximation is valid not too far from the Fermi surface, i.e. when $\left(\mathbf{v}_{\mathbf{p}} \mathbf{q}\right)_{\xi(\mathbf{p})=0} \ll E_{F}$.

It is impossible to carry out the angular averaging in (153) for a general anisotropic spectrum. Nevertheless in the following calculations of fluctuation effects in the vicinity of critical temperature only small momenta $\mathbf{v}_{\mathbf{p}} \mathbf{q} \ll$ $T$ will be involved in the integrations, so we can restrict our consideration here to this region, where one can expand the integrand in powers of $\mathbf{v}_{\mathbf{p}} \mathbf{q}$. Indeed, the presence of $\Theta\left(-\varepsilon_{1} \varepsilon_{2}\right)$ leaves the difference of the two fermionic frequencies in $(153)$ to be of the order of the temperature which permits 
this expansion. The first term in $\mathbf{v}_{\mathbf{p}} \mathbf{q}$ will evidently be averaged out, so with quadratic accuracy one can find:

$$
\mathcal{P}\left(\mathbf{q}, \varepsilon_{1}, \varepsilon_{2}\right)=2 \pi \nu \frac{\Theta\left(-\varepsilon_{1} \varepsilon_{2}\right)}{\left|\varepsilon_{1}-\varepsilon_{2}\right|}\left(1-2 \frac{\left\langle\left(\mathbf{v}_{\mathbf{p}} \mathbf{q}\right)^{2}\right\rangle_{F . S .}}{\left|\varepsilon_{1}-\varepsilon_{2}\right|^{2}}\right) .
$$

Now one can calculate the polarization operator

$$
\begin{aligned}
\Pi\left(\mathbf{q}, \Omega_{k}\right) & =T \sum_{\varepsilon_{n}} \mathcal{P}\left(\mathbf{q}, \varepsilon_{n+k}, \varepsilon_{-n}\right)= \\
& =\nu\left[\sum_{n \geq 0} \frac{1}{n+1 / 2+\frac{\left|\Omega_{k}\right|}{4 \pi T}}-2 \frac{\left\langle\left(\mathbf{v}_{\mathbf{p}} \mathbf{q}\right)^{2}\right\rangle_{F . S .}}{(4 \pi T)^{2}} \sum_{n=0}^{\infty} \frac{1}{\left(n+1 / 2+\frac{\left|\Omega_{k}\right|}{4 \pi T}\right)^{3}}\right] .
\end{aligned}
$$

The calculation of the sums in $(156)$ can be carried out in terms of the logarithmic derivatives of the $\Gamma$-function $\psi^{(n)}(x)$. It worth mentioning that the first sum is well known in the BCS theory, one can recognize in it the so-called "Cooper logarithm"; its logarithmic divergence at the upper limit $(\psi(x \gg 1) \approx \ln x)$ is cut off by the Debye energy $\left(N_{\max }=\frac{\omega_{D}}{2 \pi T}\right)$ and one gets:

$$
\begin{aligned}
\frac{1}{\nu} \Pi\left(\mathbf{q}, \Omega_{k}\right)= & \psi\left(\frac{1}{2}+\frac{\left|\Omega_{k}\right|}{4 \pi T}+\frac{\omega_{D}}{2 \pi T}\right)-\psi\left(\frac{1}{2}+\frac{\left|\Omega_{k}\right|}{4 \pi T}\right)- \\
& -\frac{\left\langle\left(\mathbf{v}_{\mathbf{p}} \mathbf{q}\right)^{2}\right\rangle_{F . S .}}{(4 \pi T)^{2}} \psi^{\prime \prime}\left(\frac{1}{2}+\frac{\left|\Omega_{k}\right|}{4 \pi T}\right) .
\end{aligned}
$$

The critical temperature in the BCS theory is determined as the temperature $T_{c}$ at which the pole of $L\left(0,0, T_{c}\right)$ occurs

$$
\begin{aligned}
& L^{-1}\left(\mathbf{q}=0, \Omega_{k}=0, T_{c}\right)=g^{-1}-\Pi\left(0,0, T_{c}\right)=0, \\
& T_{c}=\frac{2 \gamma_{E}}{\pi} \omega_{D} \exp \left(-\frac{1}{\nu g}\right),
\end{aligned}
$$

where $\gamma_{E}=1.78$ is the Euler constant. Introducing the reduced temperature $\epsilon=\ln \left(\frac{T}{T_{c}}\right)$ one can write the propagator as

$$
L^{-1}\left(\mathbf{q}, \Omega_{k}\right)=-\nu\left[\epsilon+\psi\left(\frac{1}{2}+\frac{\left|\Omega_{k}\right|}{4 \pi T}\right)-\psi\left(\frac{1}{2}\right)-\frac{\left\langle\left(\mathbf{v}_{\mathbf{p}} \mathbf{q}\right)^{2}\right\rangle_{F . S .}}{(4 \pi T)^{2}} \psi^{\prime \prime}\left(\frac{1}{2}+\frac{\left|\Omega_{k}\right|}{4 \pi T}\right)\right] .
$$

We found (159) for bosonic imaginary Matsubara frequencies $i \Omega_{k}=2 \pi i T k$. These frequencies are necessary for the calculation of fluctuation contributions to any thermodynamical characteristics of the system.

In the vicinity of the transition point one can restrict oneself in summations of the expressions with $L\left(\mathbf{q}, \Omega_{k}\right)$ over Matsubara frequencies to the so-called static approximation, taking into account the term with $\Omega_{k}=0$ 
only, which turns out to be the most singular term in $\epsilon \ll 1$. This approximation physically means that the product of Heisenberg field operators $\widetilde{\psi}_{p, \sigma} \widetilde{\psi}_{-p,-\sigma}$ appears here like a classical field $\Psi$, which in the phenomenological approach describes the Cooper pair wave function and in the vicinity of critical temperature is proportional to the fluctuation order parameter. Having in mind namely this GL region of temperatures we restricted ourselves above by the assumption of small momenta $\mathbf{v}_{\mathbf{p}} \mathbf{q} \ll T$. In these conditions the static propagator reduces to

$$
L(\mathbf{q}, 0)=-\frac{1}{\nu} \frac{1}{\epsilon+\xi^{2} \mathbf{q}^{2}}
$$

With an accuracy of a numerical factor and the total sign this correlator coincides with the expression for $\left\langle\left|\Psi_{\mathbf{q}}\right|^{2}\right\rangle$. By this expression we also have finally obtained the microscopic value of the coherence length $\xi$ for a clean superconductor with an isotropic $D$-dimensional Fermi surface which was often mentioned previously (compare with (5))

$$
\xi_{(D)}^{2}=\frac{7 \zeta(3) \mathbf{v}_{\mathbf{F}}^{2}}{16 D \pi^{2} T^{2}} .
$$

In order to describe the fluctuation contributions to transport phenomena one has to start from the analytical continuation of the propagator (159) from the discrete set of $\Omega_{k} \geq 0$ to the whole upper half-plane of imaginary frequencies. The analytical properties of $\psi^{(n)}(x)$-functions (which have poles at $x=0,-1,-2 \ldots)$ permit one to obtain the retarded propagator $L^{R}(\mathbf{q},-i \Omega)$ by simple substitution $i \Omega_{k} \rightarrow \Omega$. For small $\Omega \ll T$ the $\psi$-functions can be expanded in $-i \Omega / 4 \pi T$ and the propagator acquires the simple pole form :

$$
L^{R}(\mathbf{q}, \Omega)=-\frac{1}{\nu} \frac{1}{-\frac{i \pi}{8 T} \Omega+\epsilon+\xi^{2} \mathbf{q}^{2}}=\frac{8 T}{\pi \nu} \frac{1}{i \Omega-\left(\tau_{G L}^{-1}+\frac{8 T}{\pi} \xi^{2} \mathbf{q}^{2}\right)} .
$$

This expression provides us with the microscopic value of the GL relaxation time $\tau_{G L}=\frac{\pi}{8\left(T-T_{c}\right)}$, widely used above in the phenomenological theory. Moreover, comparison of the microscopically derived (162) with the phenomenological expressions (86), (90) and (109) shows that $\alpha T_{c}=\nu$ and $\gamma_{G L}=\pi \nu / 8 T_{c}$.

In evaluating $L\left(\mathbf{q}, \Omega_{k}\right)$ we neglected the effect of fluctuations on the oneelectron Green functions. This is correct when fluctuations are small, i.e. not too near to the transition temperature. The exact criterion of this approximation will be discussed in the following.

\subsection{Superconductor with impurities}

Account for impurities. In order to study fluctuations in real systems like superconducting alloys or high temperature superconductors one has to 


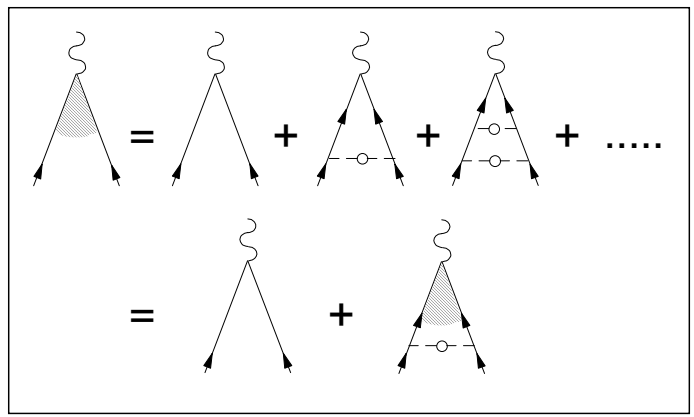

Fig. 7. The equation for the vertex part $\lambda\left(q, \omega_{1}, \omega_{2}\right)$ in the ladder approximation. Solid lines correspond to bare one-electron Green functions and dashed lines to the impurity random potential correlators.

perform an impurity average in the graphical equation for the fluctuation propagator (see Fig. 6). This procedure can be done in the framework of the Abrikosov-Gorkov approach [92], which we shortly recall below.

Let us start from the equation for the electron Green function in the potential of impurities $U(\mathbf{r})$ :

$$
(E-U(\mathbf{r})-\widehat{H}) G_{E}\left(\mathbf{r}, \mathbf{r}^{\prime}\right)=\delta\left(\mathbf{r}-\mathbf{r}^{\prime}\right)
$$

If we solve this equation using the perturbation theory for the impurity potential and average the solution, then the average product of two Green functions, can be presented as series, each term of which is associated with a graph drawn according the rules of diagrammatic technique (see Fig. 1). In this technique solid lines correspond to bare Green functions and dashed lines to random potential correlators. We assume that the impurity system random potential $U(r)$ is distributed according to the Gauss $\delta$-correlated law. Then all the correlators can be represented as the products of pair correlators

$$
\langle U(r)\rangle=0,\left\langle U(r) U\left(r^{\prime}\right)\right\rangle=\left\langle U^{2}\right\rangle \delta\left(r-r^{\prime}\right),
$$

where the angle brackets denote averaging over the impurity configuration. Equation 164 corresponds to the Born approximation for the electron interaction with short range impurities, and $\left\langle U^{2}\right\rangle=C_{i m p}\left(\int V(\mathbf{r}) d \mathbf{r}\right)^{2}$ where $C_{i m p}$ is the impurity concentration and $V(\mathbf{r})$ is the potential of the single impurity. 
In conductors (far enough from the metal-insulator transition) the mean free path is much greater than the electron wavelength $l \gg \lambda=2 \pi / p_{F}$ (which in practice means the mean free path up to tens of interatomic distances). As is well known 92] for the electron spectra with dimensionality $D>1$ the angular integration in momentum space reduces considerably the contribution of the diagrams with intersecting impurity lines what permits to omit them to the leading approximation in $\left(p_{F} l\right)^{-1}$. In this approximation the one-electron Green function keeps the same form as the bare one (149) with the only substitution

$$
\varepsilon_{n} \Rightarrow \widetilde{\varepsilon}_{n}=\varepsilon_{n}+\frac{1}{2 \tau} \operatorname{sign}\left(\varepsilon_{n}\right),
$$

where $1 / \tau=2 \pi \nu\left\langle U^{2}\right\rangle$ is the frequency of elastic collisions.

Another effect of the coherent scattering on the same impurity by both electrons forming a Cooper pair is the renormalization of the vertex part $\lambda\left(\mathbf{q}, \varepsilon_{1}, \varepsilon_{2}\right)$ in the particle-particle channel. Let us demonstrate the details of its calculation. The renormalized vertex $\lambda\left(\mathbf{q}, \varepsilon_{1}, \varepsilon_{2}\right)$ is determined by a graphical equation of the ladder type (see Fig. 7 ). Here after the averaging over the impurity configurations the value $\left\langle U^{2}\right\rangle=\frac{1}{2 \pi \nu \tau}$ is associated with the dashed line. In the momentum representation this, generally speaking, integral equation is reduced to the algebraic one

$$
\lambda^{-1}\left(\mathbf{q}, \varepsilon_{1}, \varepsilon_{2}\right)=1-\frac{1}{2 \pi \nu \tau} \mathcal{P}\left(\mathbf{q}, \widetilde{\varepsilon}_{1}, \widetilde{\varepsilon}_{2}\right),
$$

where $\mathcal{P}\left(\mathbf{q}, \widetilde{\varepsilon}_{1}, \widetilde{\varepsilon}_{2}\right)$ was defined above by (153).

Now one has to perform a formal averaging of the general expression (153) over the Fermi surface $\left(\langle\ldots\rangle_{F . S .}\right)$. Restricting consideration to small momenta

$$
\left.\Delta \xi(\mathbf{q}, \mathbf{p})\right|_{|\mathbf{p}|=p_{F}} \ll\left|\widetilde{\varepsilon}_{1}-\widetilde{\varepsilon}_{2}\right| .
$$

the calculation of $\lambda\left(\mathbf{q}, \omega_{1}, \omega_{2}\right)$ for the practically important case of an arbitrary spectrum can be done analogously to 155 . Indeed, expanding the denominator of (153) one can find

$$
\lambda\left(\mathbf{q}, \omega_{1}, \omega_{2}\right)=\frac{\left|\widetilde{\varepsilon}_{1}-\widetilde{\varepsilon}_{2}\right|}{\left|\varepsilon_{1}-\varepsilon_{2}\right|+\frac{\left\langle\left(\Delta \xi(\mathbf{q}, \mathbf{p})|| \mathbf{p} \mid p_{F}\right)^{2}\right\rangle_{F . S .}}{\tau\left|\hat{\omega}_{1}-\hat{\omega}_{2}\right|^{2}} \Theta\left(-\varepsilon_{1} \varepsilon_{2}\right)} .
$$

It is easy to see that assumed restriction on momenta is not too severe and is almost always satisfied in calculations of fluctuation effects at temperatures near $T_{c}$. In this region of temperatures the effective propagator momenta are determined by $|\mathbf{q}|_{\text {eff }} \sim\left[\xi^{G L}(T)\right]^{-1}=\xi^{-1} \sqrt{\epsilon} \ll \xi^{-1}$, while the Green function q-dependence becomes important for much larger momenta $q \sim$ $\min \left\{\xi^{-1}, l^{-1}\right\}$, which is equivalent to the limit of the condition (167). 
The average in (168) can be calculated for some particular types of spectra. For example in the cases of $2 D$ and $3 D$ isotropic spectra it is expressed in terms of the diffusion coefficient $\mathcal{D}_{(D)}$ :

$$
\left\langle\left(\left.\Delta \xi(\mathbf{q}, \mathbf{p})\right|_{|\boldsymbol{p}|=p_{F}}\right)^{2}\right\rangle_{F . S .(D)}=\tau^{-1} \mathcal{D}_{(D)} q^{2}=\frac{v_{F}^{2} q^{2}}{D} .
$$

Another important example is already familiar case of quasi-two-dimensional electron motion in a layered metal:

$$
\xi(\mathbf{p})=E\left(\mathbf{p}_{\|}\right)+J \cos \left(p_{z} s\right)-E_{F},
$$

where $E\left(\mathbf{p}_{\|}\right)=\mathbf{p}_{\|}^{2} /(2 m), \mathbf{p} \equiv\left(\mathbf{p}_{\|}, p_{z}\right), \mathbf{p}_{\|} \equiv\left(p_{x}, p_{y}\right), J$ is the effective nearest-neighbor interlayer hopping energy for quasiparticles. We note that $J$ characterizes the width of the band in the $c$-axis direction taken in the strong-coupling approximation and can be identified with the effective energy of electron tunneling between planes (see (63) and footnote 14). The Fermi surface, defined by the condition $\xi(\mathbf{p})=0$, is a corrugated cylinder (see Fig. 8). In this case the average (169) is written in a more sophisticated form:

$$
\left\langle\left(\left.\Delta \xi(\mathbf{q}, \mathbf{p})\right|_{|\boldsymbol{p}|=p_{F}}\right)^{2}\right\rangle_{F . S .}=\frac{1}{2}\left(v_{F}^{2} \mathbf{q}^{2}+4 J^{2} \sin ^{2}\left(q_{z} s / 2\right)\right)=\tau^{-1} \widehat{\mathcal{D}} q^{2},
$$

where we have introduced the definition of the generalized diffusion operator $\widehat{\mathcal{D}}$ in order to deal with an arbitrary anisotropic spectrum.

Propagator. In Section 4, in the process of the microscopic derivation of the TDGL equation, the fluctuation propagator was introduced. This object is of first importance for the microscopic fluctuation theory and it has to be generalized for the case of an impure metal with an anisotropic electron spectrum. This is easy to do using the averaging procedure presented in the previous Section. Formally it is enough to use in equation (150) the polarization operator $\Pi\left(\mathbf{q}, \Omega_{k}\right)$ averaged over impurity positions, which can be expressed in terms of $\mathcal{P}\left(\mathbf{q}, \widetilde{\varepsilon}_{n+k}, \widetilde{\varepsilon}_{-n}\right)$ introduced above:

$$
\begin{aligned}
\Pi\left(\mathbf{q}, \Omega_{k}\right) & =T \sum_{\omega_{n}} \lambda\left(q, \varepsilon_{n+k}, \varepsilon_{-n}\right) \mathcal{P}\left(\mathbf{q}, \widetilde{\omega}_{n+k}, \widetilde{\omega}_{-n}\right)= \\
& =T \sum_{\omega_{n}} \frac{1}{\left[\mathcal{P}\left(\mathbf{q}, \widetilde{\varepsilon}_{n+k}, \widetilde{\varepsilon}_{-n}\right)\right]^{-1}-\frac{1}{2 \pi \nu \tau}} .
\end{aligned}
$$

For relatively small $\mathbf{q}\left(\left.\Delta \xi(\mathbf{q}, \mathbf{p})\right|_{|E(\mathbf{p})|=E_{F}} \ll\left|\widetilde{\varepsilon}_{n+k}-\widetilde{\varepsilon}_{-n}\right| \sim \max \left\{T, \tau^{-1}\right\}\right)$ and $\Omega \ll T$ one can find an expression for the fluctuation propagator, which can be useful in studies of fluctuation effects near $T_{c}(\epsilon \ll 1)$ for the dirty and intermediate but not very clean case $(T \tau \ll 1 / \sqrt{\epsilon})$. Expanding (168) in 


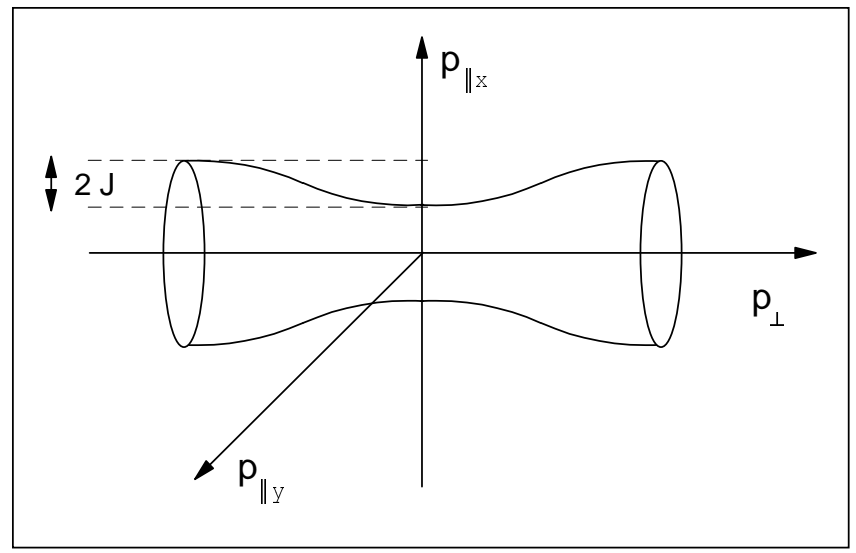

Fig. 8. The Fermi surface in the form of a corrugated cylinder

powers of $\left(\left.\Delta \xi(\mathbf{q}, \mathbf{p})\right|_{|E(\mathbf{p})|=E_{F}} /\left|2 \widetilde{\varepsilon}_{n}+\Omega_{k}\right|\right)^{2}$ it is possible write $L^{R}(q, \Omega)$ in a form almost completely coinciding with $\operatorname{Exp}$ (162):

$$
L^{R}(\mathbf{q}, \Omega)=-\frac{1}{\nu} \frac{1}{\epsilon-i \frac{\pi \Omega}{8 T}+\xi^{2}(T \tau) \mathbf{q}^{2}} .
$$

Let us stress that the phenomenological coefficient $\gamma_{G L}$ turns out to be equal to the same value $\frac{\pi \nu}{8 T}$ as in clean case, and hence does not depend on the impurity concentration. The only difference in comparison with the clean case is in appearance of a dependence of the natural effective coherence length on the elastic relaxation time. In the isotropic D-dimensional case it can be written as

$$
\begin{aligned}
\xi_{(D)}^{2}(T \tau)= & (4 m \alpha T)^{-1}=\eta_{(D)}= \\
& -\frac{\tau^{2} v_{F}^{2}}{D}\left[\psi\left(\frac{1}{2}+\frac{1}{4 \pi T \tau}\right)-\psi\left(\frac{1}{2}\right)-\frac{1}{4 \pi T \tau} \psi^{\prime}\left(\frac{1}{2}\right)\right]
\end{aligned}
$$

(we introduced here the parameter $\eta_{(D)}$ frequently used in the microscopic theory) 27 .

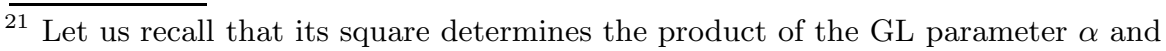
the Cooper pair mass entering in the GL functional. In clean case we supposed the letter equal to two free electron masses and defined $\alpha$ in accordance with 
The generalization of (173) for the case of a layered electronic spectrum is evident:

$$
L^{R}(q, \Omega)=-\frac{1}{\nu} \frac{1}{\epsilon-i \frac{\pi \Omega}{8 T}+\eta_{(2)} \mathbf{q}_{\|}^{2}+r \sin ^{2}\left(q_{z} s / 2\right)} .
$$

One has to remember that the Exp. (173) was derived in the assumption of small momenta $\left.\Delta \xi(\mathbf{q}, \mathbf{p})\right|_{|E(\mathbf{p})|=E_{F}} \ll\left|\widetilde{\varepsilon}_{n+k}-\widetilde{\varepsilon}_{-n}\right| \sim \max \left\{T, \tau^{-1}\right\}$, so the range of its applicability is restricted to the GL region of temperatures $\epsilon=\ln \left(\frac{T}{T_{c}}\right) \ll 1$, where the integrands of diagrammatic expressions have singularities at small momenta of the Cooper pair center of mass.

Finally let us express the Ginzburg-Levanyuk parameter for the important $2 D$ case in terms of the microscopic parameter $\eta_{(2)}$. In accordance with (37) and the definition (174):

$$
G i_{(2)}(T \tau)=\frac{7 \zeta(3)}{16 \pi^{2}} \frac{1}{m T_{c} \eta_{(2)}(T \tau)} .
$$

One can see that this general definition in the limiting cases of a clean and dirty metal results in the same values $G i_{(2 c)}$ and $G i_{(2 d)}$ as was reported in Table 1.

\subsection{Microscopic theory of fluctuation conductivity of layered superconductor}

Qualitative discussion of different fluctuation contributions In Section 4 the direct fluctuation effect on conductivity, related with the charge transfer by means of fluctuation Cooper pairs, was discussed in details. Nevertheless, below in this Section we return to its discussion and will demonstrate its calculation by means of the microscopic theory. This will be done in purpose to prepare the basis for studies of the Aslamazov-Larkin contribution in the variety of physical values like magnetoconductivity near the upper critical field, conductivity far from transition point, fluctuation conductivity in ultra-clean limit, Hall conductivity etc.

Microscopic approach permits also to calculate the cited above indirect fluctuation effects like so called DOS and MT contributions. We will start now from their qualitative discussion.

The important consequence of the presence of fluctuating Cooper pairs above $T_{c}$ is the decrease of the one-electron density of states at the Fermi level. Indeed, if some electrons are involved in pairing they can not simultaneously

(18). As we just have seen in the case of the impure superconductor $\xi$ depends on impurity concentration and this dependence, in principle, can be attributed both to $\alpha$ or $m$. For our further purposes it is convenient to leave $\alpha$ in the same form (18) as in the case of a clean superconductor. The Cooper pair mass in this case becomes dependent on the electron mean free pass what physically can be attributed to the diffusion motion of the electrons forming the pair. 
participate in charge transfer and heat capacity as single-particle excitations. Nevertheless, the total number of the electronic states can not be changed by the Cooper interaction, and only a redistribution of the levels along the energy axis is possible 93,94. In this sense one can speak about the opening of a fluctuation pseudo-gap at the Fermi level. The decrease of the oneelectron density of states at the Fermi level leads to a reduction of the normal state conductivity. This, indirect, fluctuation correction to the conductivity is called the density of states (DOS) contribution and it appears side by side with the paraconductivity (or Aslamazov-Larkin contribution). It has the opposite (negative) sign and turns out to be much less singular in $\left(T-T_{c}\right)^{-1}$ in comparison with the AL contribution, so that in the vicinity of $T_{c}$ it was usually omitted. However, in many cases [29,95, 96, 97, 98, 99, when for some special reasons the main, most singular, corrections are suppressed, the DOS correction becomes of major importance. Such a situation takes place in many cases of actual interest (quasiparticle current in tunnel structures, c-axis transport in strongly anisotropic high temperature superconductors, NMR relaxation rate, thermoelectric power).

The correction to the normal state conductivity above the transition temperature related with the fluctuation DOS renormalization for the dirty superconductor can be evaluated qualitatively. Indeed, the fact that some electrons $\left(\Delta \mathcal{N}_{e}\right.$ per unit volume) participate in fluctuation Cooper pairing means that the effective number of carriers taking part in one-electron charge transfer diminishes leading to a decrease of conductivity (we deal here with the longitudinal component):

$$
\delta \sigma_{x x}^{D O S}=-\frac{\Delta \mathcal{N}_{e} e^{2} \tau}{m}=-\frac{2 n_{s} e^{2} \tau}{m}
$$

where $n_{s}$ is the superfluid density coinciding with the Cooper pairs concentration. The latter can be identified with the average value of the square of the order parameter modulus already calculated as the correlator

$$
\left\langle\Psi^{*}(0) \Psi(\mathbf{r})\right\rangle\left(T>T_{c 0}\right)=\sum_{\mathbf{k}}\left\langle\left|\Psi_{k}\right|^{2}\right\rangle \exp (i \mathbf{k r})=\frac{1}{4 \pi \alpha \xi^{2}} \mathbf{K}_{0}\left(\frac{r}{\xi(\epsilon)}\right)
$$

with $r \sim \xi$. For the $2 D$ case, which is of the most interest to us, one finds:

$$
n_{s}=\frac{1}{4 \pi \alpha \xi^{2}} \frac{1}{s} \mathbf{K}_{0}(\sqrt{\epsilon})=\frac{7 \zeta(3)}{\pi^{4} v_{F}^{2}} \frac{E_{F}}{s \tau} \ln \frac{1}{\epsilon},
$$

where we have used the explicit expression (18) for $\alpha$ and $\xi$. As we will see the corresponding expression for the fluctuation DOS correction to conductivity (177) coincides with the accuracy of 2 with the microscopic expression (191) which will be carried out below.

The third, purely quantum, fluctuation contribution is generated by the coherent scattering of the electrons forming a Cooper pair on the same elastic 
impurities. This is the so called anomalous Maki-Thompson (MT) contribution [6,7] which can be treated as the result of Andreev scattering of the electron by fluctuation Cooper pairs. This contribution often turns out to be important in conductivity and other transport phenomena. Its temperature singularity near $T_{c}$ is similar to that of the paraconductivity, although being extremely sensitive to electron phase-breaking processes and to the type of orbital symmetry of pairing it can be suppressed. Let us evaluate it.

The physical origin of the Maki-Thompson correction consists in the fact that the Cooper interaction of electrons with the almost opposite momenta changes the mean free path (diffusion coefficient) of electrons. As we have already seen in the previous Section the amplitude of this interaction increases drastically when $T \rightarrow T_{c}$ :

$$
g_{e f f}=\frac{g}{1-g \ln \frac{\omega_{D}}{2 \pi T}}=\frac{1}{\ln \frac{T}{T_{c}}} \approx \frac{T}{T-T_{c}}=\frac{1}{\epsilon} .
$$

What is the reason of this growth? One can say that the electrons scatter one at another in resonant way with the virtual Cooper pairs formation. Or it is possible to imagine that the electrons undergo the Andreev scattering at fluctuation Cooper pairs binding in the Cooper pair themselves. The probability of such induced pair irradiation (let us remind that Cooper pairs are Bose particles) is proportional to their number in the final state, i.e. $n(p)$ (7). For small momenta $n(p) \sim 1 / \epsilon$.

One can ask why such interaction does not manifest itself considerably far from the transition point? The matter of fact that so intensively interacts just small number of electrons with the total momentum $q \lesssim \xi^{-1}(T)$. In accordance with the Heisenberg principle the minimal distance between such electrons is of the order of $\sim \xi(T)$. From the other hand such electrons in purpose to interact have to approximate one another up to the distance of the Fermi length $\lambda_{F} \sim 1 / p_{F}$. The probability of such event may be estimated in the spirit of the self-intersection trajectories contribution evaluation in the weak localization theory [100, 102$]$.

In the process of diffusion motion the distance between two electrons increases with the time growth in accordance with the Einstein law: $R(t) \sim$ $(D t)^{1 / 2}$. Hence the scattering probability

$$
W \sim \int_{t_{\min }}^{t_{\max }} \frac{\lambda_{F}^{D-1}}{R^{D}(t)} v_{F} d t .
$$

The lower limit of the integral can be estimated from the condition $R\left(t_{\min }\right)$ $\sim \xi(T)$ (only such electrons interact in the resonant way). The upper limit is determined by the phase breaking time $\tau_{\varphi}$ since for larger time intervals the phase coherence, necessary for the pair formation, is broken. In result the relative correction to conductivity due to such processes is equal to the 
product of the scattering probability on the effective interaction constant: $\delta \sigma^{M T} / \sigma=W g_{\text {eff }}$. In the $2 D$ case

$$
\delta \sigma^{M T} \sim \frac{e^{2}}{8 \epsilon} \ln \frac{D \tau_{\varphi}}{\xi^{2}(T)} .
$$

This result will be confirmed below in the frameworks of the microscopic consideration.

Generalities Let us pass to the microscopic calculation of the fluctuation conductivity of the layered superconductor. We begin by discussing the quasiparticle normal state energy spectrum. While models with several conducting layers per unit cell and with either intralayer or interlayer pairing have been considered 103, it has been shown 104 that all of these models give rise to a Josephson pair potential that is periodic in $k_{z}$, the wave-vector component parallel to the $c$-axis, with period $s$, the $c$-axis repeat distance. While such models differ in their superconducting densities of states, they all give rise to qualitatively similar fluctuation propagators, which differ only in the precise definitions of the parameters and in the precise form of the Josephson coupling potential. Ignoring the rather unimportant differences between such models in the Gaussian fluctuation regime above $T_{c}(H)$, we therefore consider the simplest model of a layered superconductor, in which there is one layer per unit cell, with intralayer singlet $s$-wave pairing. These assumptions lead to the simple spectrum (170) and hence to a Fermi surface having the form of a corrugated cylinder (see Fig.8).

Some remarks regarding the normal-state quasiparticle momentum relaxation time are necessary. In the "old" layered superconductors the materials were generally assumed to be in the dirty limit (like $\operatorname{Ta}_{2}$ (pyridine) ${ }_{1 / 2}$ ). In the high- $T_{c}$ cuprates, however, both single crystals and epitaxial thin films are nominally in the "intermediate" regime, with $l / \xi_{x y} \approx 2-5$. In addition, the situation in the cuprates is complicated by the presence of phonons for $T \simeq T_{c} \simeq 100 K$, the nearly localized magnetic moments on the $\mathrm{Cu}^{2+}$ sites, and by other unspecified inelastic processes. In this Section we assume simple elastic intralayer scattering and restrict our consideration to the local limit in the fluctuation Cooper pair motion. This means that we consider the case of not too clean superconductors, keeping the impurity concentration $n_{i}$ and reduced temperature such that the resulting mean-free path satisfies the requirement $l<\xi_{x y}(T)=\frac{\xi_{x y}}{\sqrt{\varepsilon}}$ and the impurity vertex can be taken in the local form (168) with $\left\langle(\Delta \xi(\mathbf{q}, \mathbf{p}) \mid)^{2}\right\rangle_{F . S .}$. determined by (171). The phase-breaking time $\tau_{\varphi}$ is supposed to be much larger than $\tau$.

The most general relation between the current density $\mathbf{j}(\mathbf{r}, t)$ and vectorpotential $\mathbf{A}\left(\mathbf{r}^{\prime}, t^{\prime}\right)$ is given through the so-called electromagnetic response operator $Q_{\alpha \beta}\left(\mathbf{r}, \mathbf{r}^{\prime}, t, t^{\prime}\right)$ [92]:

$$
\mathbf{j}(\mathbf{r}, t)=\int Q_{\alpha \beta}\left(\mathbf{r}, \mathbf{r}^{\prime}, t, t^{\prime}\right) \mathbf{A}\left(\mathbf{r}^{\prime}, t^{\prime}\right) \mathbf{d} \mathbf{r}^{\prime} d t,
$$


Assuming space and time homogeneity, one can take the Fourier transform of this relation and compare it with the definition of the conductivity tensor $j_{\alpha}=\sigma_{\alpha \beta} E_{\beta}$. This permits us to express the conductivity tensor in terms of the retarded electromagnetic response operator

$$
\sigma_{\alpha \beta}(\omega)=-\frac{1}{i \omega}\left[Q_{\alpha \beta}\right]^{R}(\omega) .
$$

The electromagnetic response operator $Q_{\alpha \beta}\left(\omega_{\nu}\right)$, defined on Matsubara frequencies $\omega_{\nu}=(2 \nu+1) \pi T$, can be presented as the correlator of two exact one-electron Green functions 92] averaged over impurities and accounting for interactions, in our case the particle-particle interactions in the Cooper channel. The appropriate diagrams corresponding to the first order of perturbation theory in the fluctuation amplitude are shown in Fig. (9).

With each electromagnetic field component $A_{\alpha}$ we associate the external vertex $e v_{\alpha}(p)=e \frac{\partial \xi(p)}{\partial p_{\alpha}}$. For the longitudinal conductivity tensor elements (parallel to the layers, for which $\alpha=x, y$ ), the resulting vertex is simply $e p_{\alpha} / m$. For the $c$-axis conductivity, the vertex is given by

$$
e v_{z}(p)=e \frac{\partial \xi(p)}{\partial p_{z}}=-e J s \sin \left(p_{z} s\right) .
$$

Each solid line in the diagrams represents a one-electron Green function averaged over impurities (149), a wavy line represents a fluctuation propagator $L\left(\mathbf{q}, \Omega_{k}\right)(173)$, three-leg vertices were defined by the Exp.(168). The four-leg impurity vertex, appearing in diagrams 3-4, 9-10 of the Fig. 9, is called the Cooperon in the weak localization theory (see, for example, 105 ) It is easy to see that it differs from the above three-leg vertex only by the additional factor $(2 \pi \nu \tau)^{-1}$. We do not renormalize the current vertices: it is well known (see 92]) that this renormalization only leads to the substitution of the scattering time $\tau$ by the transport one $\tau_{t r}$. We integrate over the internal Cooper pair momentum $\mathbf{q}$ and electron momentum $\mathbf{p}$ and sum over the internal fermionic and bosonic Matsubara frequencies, with momentum and energy conservation at each internal vertex (fluctuation propagator endpoint) in the analytical expressions for the diagrams presented in Fig. (9).

After these necessary introductory remarks and definitions we pass to the microscopic calculation of the different fluctuation contributions.

Aslamazov-Larkin contribution We first examine the AL paraconductivity (diagram 1 of Fig.(9)). Actually this contribution was already studied in the Section 4 in the framework of the TDGL equation but, in order to demonstrate how the method works, we will carry out here the appropriate calculations in the microscopic approach, as was originally done by Aslamazov and Larkin [5]. 

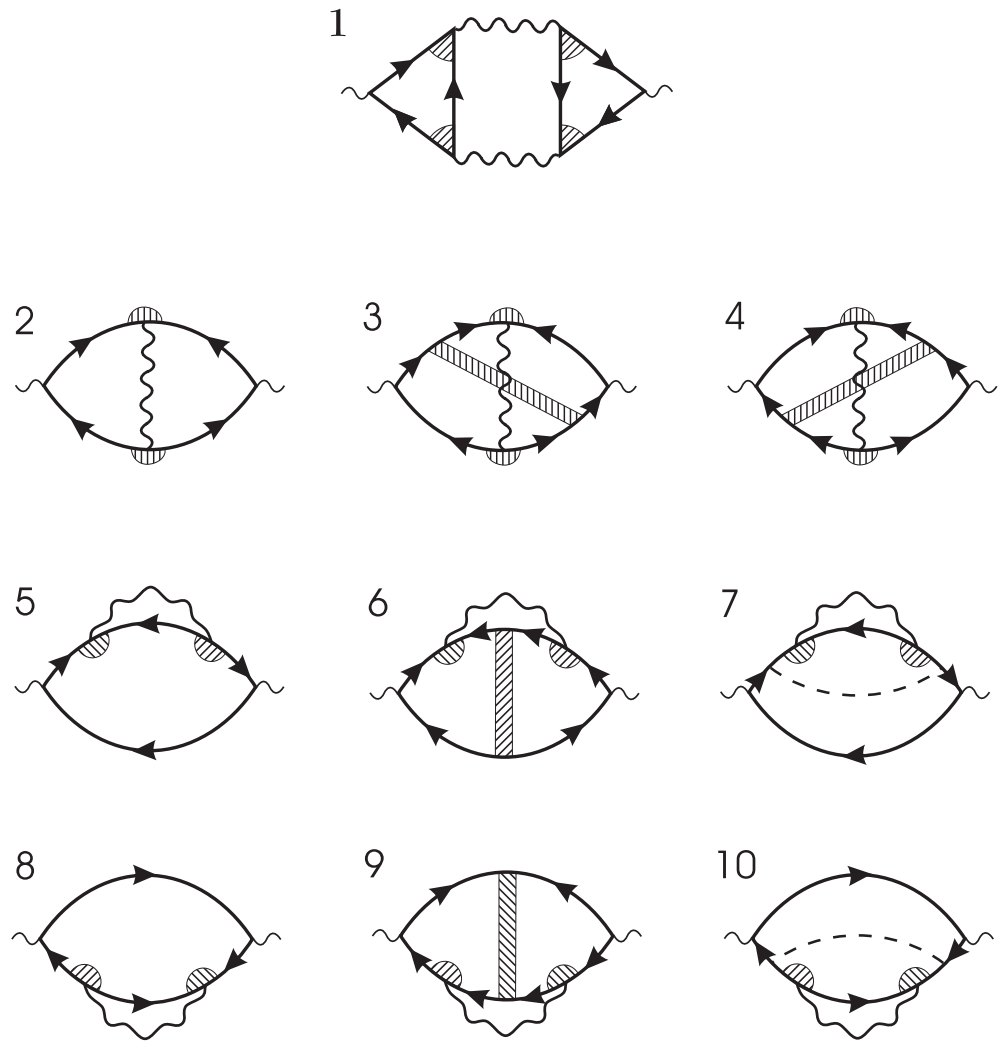

Fig. 9. Feynman diagrams for the leading-order contributions to the fluctuation conductivity. Wavy lines are fluctuation propagators, thin solid lines with arrows are impurity-averaged normal-state Green's functions, shaded semicircles are vertex corrections arising from impurities, dashed lines with central crosses are additional impurity renormalisations and shaded rectangles are impurity ladders. Diagram 1 represents the Aslamazov-Larkin term; diagrams 2-4 represent the Maki-Thompson type contributions; diagrams 5-10 arise from corrections to the normal state density of states. 
The AL contribution to the electromagnetic response operator tensor has the form:

$$
\begin{aligned}
Q_{\alpha \beta}^{A L}\left(\omega_{\nu}\right)= & 2 e^{2} T \sum_{\Omega_{k}} \int \frac{d^{3} \mathbf{q}}{(2 \pi)^{3}} B_{\alpha}\left(\mathbf{q}, \Omega_{k}, \omega_{\nu}\right) L\left(\mathbf{q}, \Omega_{k}\right) \times \\
& \times B_{\beta}\left(\mathbf{q}, \Omega_{k}, \omega_{\nu}\right) L\left(\mathbf{q}, \Omega_{k}+\omega_{\nu}\right),
\end{aligned}
$$

where the three Green function block is given by

$$
\begin{aligned}
B_{\alpha}\left(\mathbf{q}, \Omega_{k}, \omega_{\nu}\right)= & T \sum_{\varepsilon_{n}} \lambda\left(\mathbf{q}, \varepsilon_{n+\nu}, \Omega_{k}-\varepsilon_{n}\right) \lambda\left(\mathbf{q}, \varepsilon_{n}, \Omega_{k}-\varepsilon_{n}\right) \times \\
& \times \int \frac{d^{3} \mathbf{p}}{(2 \pi)^{3}} v_{\alpha}(\mathbf{p}) G\left(\mathbf{p}, \varepsilon_{n+\nu}\right) G\left(\mathbf{p}, \varepsilon_{n}\right) G\left(\mathbf{q}-\mathbf{p}, \Omega_{k}-\varepsilon_{n}\right) .
\end{aligned}
$$

Expanding $G\left(\mathbf{q}-\mathbf{p}, \Omega_{k}-\varepsilon_{n}\right)$ over $\mathbf{q}$ one find that the angular integration over the Fermi surface kills the first term and leaves nonzero the second term of the expansion only. Then the $\xi$-integration is performed by means of the Cauchy theorem. The further summation over the fermionic frequency is cumbersome, so we will show it for the example of the simplest case of a dirty superconductor with $T \tau \ll 1$. In this case the main sources of the $\varepsilon_{n}$-dependence in (183) are the $\lambda$-vertices and that originating from the Green functions can be neglected by the parameter $T \tau \ll 1$ (indeed, one can see that $\varepsilon_{n} \sim T$ are important in vertices, while in Green functions $\varepsilon_{n} \gtrsim \tau^{-1}$ only). The remaining summation in (183) is performed in the same way as was done in (173) and results in:

$$
\begin{aligned}
B_{\alpha}\left(\mathbf{q}, \Omega_{k}, \omega_{\nu}\right)= & \nu \frac{\eta_{(2)}}{v_{F}^{2}}\left\langle v_{\alpha} q_{\beta} v_{\beta}\right\rangle_{F S} \times \\
& \times \frac{8 T}{\pi \omega_{\nu}}\left[\psi\left(\frac{1}{2}+\frac{\left|\Omega_{k}\right|+\omega_{\nu}+\widehat{D} q^{2}}{4 \pi T}\right)-\psi\left(\frac{1}{2}+\frac{\left|\Omega_{k}\right|+\widehat{D} q^{2}}{4 \pi T}\right)+\right. \\
& \left.+\psi\left(\frac{1}{2}+\frac{\left|\Omega_{k+\nu}\right|+\omega_{\nu}+\widehat{D} q^{2}}{4 \pi T}\right)-\psi\left(\frac{1}{2}+\frac{|| \Omega_{k+\nu} \mid+\widehat{D} q^{2}}{4 \pi T}\right)\right] .
\end{aligned}
$$

Now let us return to the general expression for $Q_{\alpha \beta}^{A L}\left(\omega_{\nu}\right)$ and transform the $\Omega_{k}$ - summation into a contour integral, using the identity 106.

$$
T \sum_{\Omega_{k}} f\left(\Omega_{k}\right)=\frac{1}{4 \pi i} \oint_{C} d z \operatorname{coth} \frac{z}{2 T} f(-i z),
$$

where $z=i \Omega_{k}$ is a variable in the plane of complex frequency and the contour $C$ encloses all bosonic Matsubara frequencies over which the summation is carried out. In our case the contour $C$ can be chosen as a circle with radius tending to infinity (see Fig. 10): 


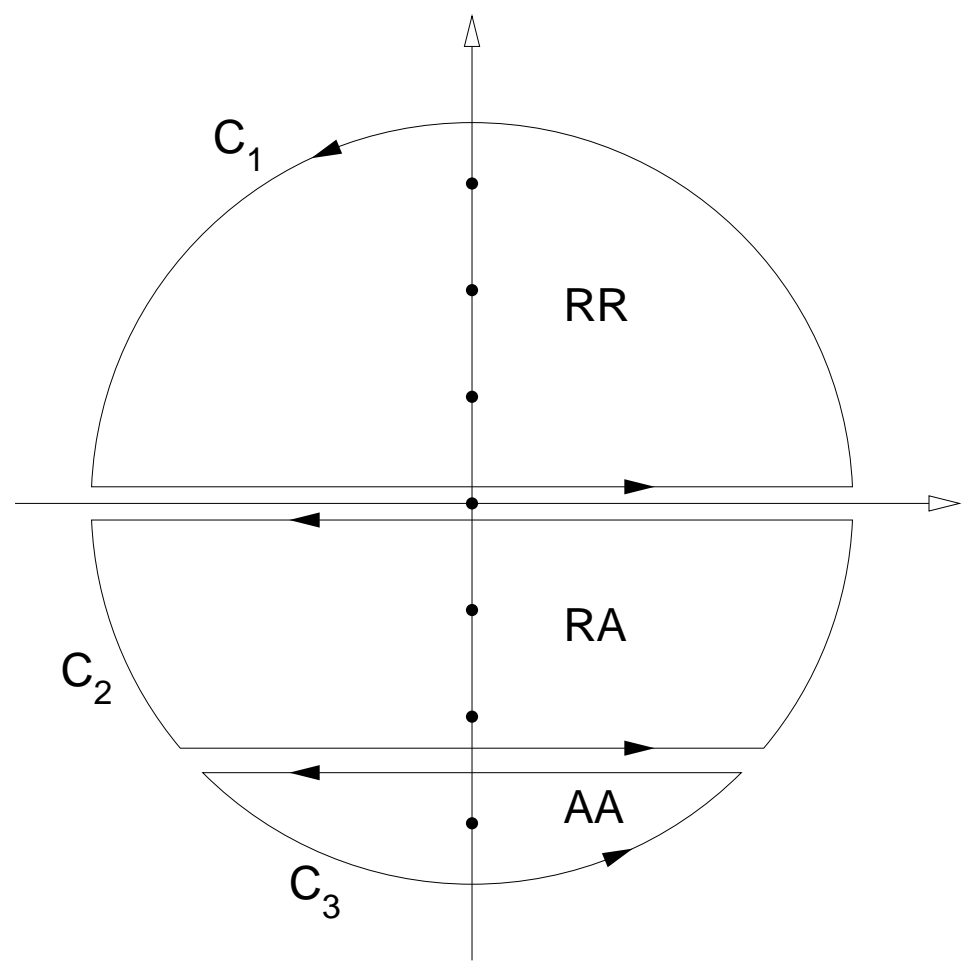

Fig. 10. The contour of integration in the plane of complex frequencies.

$$
\begin{aligned}
Q_{\alpha \beta}^{A L}\left(\omega_{\nu}\right)= & \frac{e^{2}}{2 \pi i} \int \frac{d^{3} \mathbf{q}}{(2 \pi)^{3}} \oint_{C} d z \operatorname{coth} \frac{z}{2 T} B_{\alpha}\left(\mathbf{q},-i z+\omega_{\nu},-i z\right) \times \\
& \times L(\mathbf{q},-i z) B_{\beta}\left(\mathbf{q},-i z+\omega_{\nu},-i z\right) L\left(\mathbf{q},-i z+\omega_{\nu}\right) .
\end{aligned}
$$

One can see that the integrand function in 185 ) has breaks of analyticity at the lines $\operatorname{Im} z=0$ and $\operatorname{Im} z=-i \omega_{\nu}$. Indeed, the fluctuation propagator $L\left(\mathbf{q}, \Omega_{k}\right)$ and Green function blocks $B_{\alpha}\left(\mathbf{q}, \Omega_{k}, \omega_{\nu}\right)$ were defined on the bosonic Matsubara frequencies only, while now we have to use them as functions of the continuous variable $z$. As it is well known from the properties of Green functions in the complex plane $z$, two analytical functions, related with $L\left(\mathbf{q}, \Omega_{k}\right)$, can be introduced. The first one, $L^{R}(\mathbf{q},-i z)$ (retarded), is analytic in the upper half-plane $(\operatorname{Im} z>0)$, while the second one, $L^{A}(\mathbf{q},-i z)$ (advanced), has no singularities in the lower half-plane $(\operatorname{Im} z<0)$. As we have seen above the same lines separate the domains of the analyticity of the Green function blocks, so the functions $B^{R R}, B^{R A}, B^{A A}$ analytic in each domain can be introduced (with the appropriate choices of the $\left|\Omega_{k+\nu}\right|$ and $\left|\Omega_{k}\right|$ 
signs in the arguments of the $\psi$ - functions, see (184)). This means that by cutting the $z$-plane along the lines $\operatorname{Im} z=0$ and $\operatorname{Im} z=-i \omega_{\nu}$ we can reduce the calculation of the contour integral in (185) to the sum of three integrals along the contours $C_{1}, C_{2}, C_{3}$ which enclose domains of well defined analyticity of the integrand function. The integral along the large circle evidently vanishes and the contour integral is reduced to four integrals along the cuts of the plane in Fig.10:

$$
\begin{aligned}
I\left(\mathbf{q}, \omega_{\nu}\right)= & \oint_{C_{1}+C_{2}+C_{3}} d z \operatorname{coth} \frac{z}{2 T} B_{\alpha}(\mathbf{q},-i z) L(\mathbf{q},-i z) B_{\beta}(\mathbf{q},-i z) L\left(\mathbf{q},-i z+\omega_{\nu}\right)= \\
= & \int_{-\infty}^{\infty} d z \operatorname{coth} \frac{z}{2 T} L^{R}\left(\mathbf{q},-i z+\omega_{\nu}\right)\left[B_{\alpha}^{R R} B_{\beta}^{R R} L^{R}(\mathbf{q},-i z)-\right. \\
& \left.-B_{\alpha}^{R A} B_{\beta}^{R A} L^{A}(\mathbf{q},-i z)\right]+\int_{-\infty-i \omega_{\nu}}^{\infty-i \omega_{\nu}} d z \operatorname{coth} \frac{z}{2 T} L^{A}(\mathbf{q},-i z) \times \\
& {\left[B_{\alpha}^{R A} B_{\beta}^{R A} L^{R}\left(\mathbf{q},-i z+\omega_{\nu}\right)-B_{\alpha}^{A A} B_{\beta}^{A A} L^{A}\left(\mathbf{q},-i z+\omega_{\nu}\right)\right] . . }
\end{aligned}
$$

Now one can shift the variable in the last integral to $z=z^{\prime}-i \omega_{\nu}$, take into account that $i \omega_{\nu}$ is the period of $\operatorname{coth} \frac{z}{2 T}$ and get an expression analytic in $i \omega_{\nu} \rightarrow \omega$.

In the vicinity of $T_{c}$, due to the pole structure of the fluctuation propagators in (182), the leading contribution to the electromagnetic response operator $Q_{\alpha \beta}^{A L(R)}$ arises from them rather than from the frequency dependence of the vertices $B_{\alpha}$, so we can neglect the $\Omega_{k^{-}}$and $\omega_{\nu}$-dependencies of the Green functions blocks and use the expression for $B_{\alpha}(\mathbf{q}, 0,0)$ valid for small $\mathbf{q}_{a b}$ only:

$$
B_{\alpha}(\mathbf{q})=-2 \nu \frac{\eta_{(2)}}{v_{F}^{2}}\left\{\begin{array}{c}
v_{F}^{2} q_{\alpha}, \quad \alpha=x, y \\
s J^{2} \sin q_{z} s, \quad \alpha=z
\end{array} .\right.
$$

Detailed calculations demonstrate that this result can be generalized to an arbitrary impurity concentration just by using the expression (174) for $\eta_{(2)}$. Finally:

$$
\begin{aligned}
Q_{\alpha \beta}^{A L(R)}(\omega)=\frac{2 e^{2}}{\pi} \int \frac{d^{3} q}{(2 \pi)^{3}} B_{\alpha}(\mathbf{q}) B_{\beta}(\mathbf{q}) & \int_{-\infty}^{\infty} d z \operatorname{coth}\left(\frac{z}{2 T}\right)\left[L^{R}(\mathbf{q},-i z-i \omega)\right. \\
& \left.+L^{A}(\mathbf{q},-i z+i \omega)\right] \operatorname{Im} L^{R}(\mathbf{q},-i z)
\end{aligned}
$$

Being interested here in the d.c. conductivity one can expand the integrand function in $\omega$. It is possible to show that the zeroth order term is cancelled by the same type contributions from all other diagrams (this cancellation confirms the absence of anomalous diamagnetism above the critical temperature). The remaining integral can be integrated by parts and then 
carried out taking into account that the contribution most singular in $\epsilon$ comes from the region $z \sim \epsilon \ll T$ :

$$
\begin{aligned}
\sigma_{x x}^{A L} & =\frac{e^{2}}{2 \pi T} \int \frac{d^{3} \mathbf{q}}{(2 \pi)^{3}} B_{x, z}^{2}(\mathbf{q}, 0,0) \int_{-\infty}^{\infty} \frac{d z}{\sinh ^{2} \frac{z}{2 T}}\left[\operatorname{Im} L^{R}(\mathbf{q},-i z)\right]^{2}= \\
& =\frac{\pi^{2} e^{2} \eta_{(2)}^{2}}{s} \int \frac{d^{2} \mathbf{q}}{(2 \pi)^{2}} \frac{\mathbf{q}^{2}}{\left[\left(\eta_{(2)} \mathbf{q}^{2}+\epsilon\right)\left(\eta_{(2)} \mathbf{q}^{2}+\epsilon+r\right)\right]^{3 / 2}} \\
& =\frac{e^{2}}{16 s} \frac{1}{[\epsilon(\epsilon+r)]^{1 / 2}} \rightarrow \frac{e^{2}}{16 s}\left\{\begin{array}{c}
1 / \sqrt{\epsilon r}, \epsilon \ll r \\
1 / \epsilon, \quad \epsilon \gg r .
\end{array}\right.
\end{aligned}
$$

where the Lawrence-Doniach anisotropy parameter $r$ [27] was already defined by 63 .

In the same way one can evaluate the AL contribution to the transverse fluctuation conductivity 107,95, 108:

$$
\begin{aligned}
\sigma_{z z}^{A L} & =\frac{\pi e^{2} s r^{2}}{32} \int \frac{d^{2} \mathbf{q}}{(2 \pi)^{2}} \frac{1}{\left[\left(\eta_{(2)} \mathbf{q}^{2}+\epsilon\right)\left(\eta_{(2)} \mathbf{q}^{2}+\epsilon+r\right)\right]^{3 / 2}}= \\
& =\frac{e^{2} s}{32 \eta_{(2)}}\left(\frac{\epsilon+r / 2}{[\epsilon(\epsilon+r)]^{1 / 2}}-1\right) \rightarrow \frac{e^{2} s}{64 \eta_{(2)}}\left\{\begin{array}{c}
\sqrt{r / \epsilon}, \text { for } \epsilon \ll r \\
(r / 2 \epsilon)^{2}, \text { for } \epsilon \gg r
\end{array} .\right.
\end{aligned}
$$

Note, that contrary to the case of in-plane conductivity, the critical exponent for $\sigma_{z z}$ above the Lawrence-Doniach crossover temperature $T_{L D}$ (for which $\left.\epsilon\left(T_{L D}\right)=r\right)$ is 2 instead of 1 , so the crossover occurs from the $0 \mathrm{D}$ to $3 \mathrm{D}$ regimes. This is related with the tunneling (so from the band structure point of view effectively zero dimensional) character of electron motion along the c-axis.

Contributions from fluctuations of the density of states In original paper of Aslamazov and Larkin [5] the most singular AL contribution to conductivity, heat capacity and other properties of a superconductor above the critical temperature was considered. The diagrams of the type 5-6 were pictured and correctly evaluated as less singular in $\epsilon$. Nevertheless the specific form of the AL contribution to the transverse conductivity of a layered superconductor, which may be considerably suppressed for small interlayer transparency, suggested to re-examine the contributions from diagrams 5-10 of Fig. 9 which are indeed less divergent in $\epsilon$, but turn out to be of lower order in the transmittance and of the opposite sign with respect to the AL one 95.96. These, so-called DOS, diagrams describe the changes in the normal Drude-type conductivity due to fluctuation renormalization of the normal quasiparticles density of states above the transition temperature (see Section 8.5). In the dirty limit, the calculation of contributions to the longitudinal fluctuation conductivity $\sigma_{x x}$ from such diagrams was discussed in [109, 99. Contrary to the case of the AL contribution, the in-plane and out-of-plane components of the DOS contribution differ only in the square of the ratio of 
effective Fermi velocities in the parallel and perpendicular directions. This allows us to calculate both components simultaneously. The contribution to the fluctuation conductivity due to diagram 5 is

$$
\begin{aligned}
Q_{\alpha \beta}^{5}\left(\omega_{\nu}\right)= & 2 e^{2} T \sum_{\Omega_{k}} \int \frac{d^{3} \mathbf{q}}{(2 \pi)^{3}} L\left(\mathbf{q}, \Omega_{k}\right) T \sum_{\varepsilon_{n}} \lambda^{2}\left(\mathbf{q}, \varepsilon_{n}, \Omega_{k}-\varepsilon_{n}\right) \times \\
& \int \frac{d^{3} \mathbf{p}}{(2 \pi)^{3}} v_{\alpha}(\mathbf{p}) v_{\beta}(\mathbf{p}) G^{2}\left(\mathbf{p}, \varepsilon_{n}\right) G\left(\mathbf{q}-\mathbf{p}, \Omega_{k}-\varepsilon_{n}\right) G\left(\mathbf{p}, \varepsilon_{n+\nu}\right),
\end{aligned}
$$

and diagram 6 gives an identical contribution. Evaluation of the integrations over the in-plane momenta $\mathbf{p}$ and the summation over the internal frequencies $\varepsilon_{n}$ are straightforward. Treatment of the other internal frequencies $\Omega_{k}$ is less obvious, but in order to obtain the leading singular behavior in the vicinity of transition it suffices to set $\Omega_{k}=0$ [99]. After integration over $q_{z}$, we have [95, 97 :

$$
\begin{aligned}
\sigma_{\alpha \beta}^{5+6} & =-\frac{\pi e^{2}}{2 s} A_{\alpha \beta} \kappa_{1} \eta_{(2)} \int_{|\mathbf{q}| \leq \xi^{-1}} \frac{d^{2} \mathbf{q}}{(2 \pi)^{2}} \frac{1}{\left[\left(\epsilon+\eta_{(2)} \mathbf{q}^{2}\right)\left(\epsilon+r+\eta_{(2)} \mathbf{q}^{2}\right)\right]^{1 / 2}} \\
& \approx-\frac{e^{2} \kappa_{1}}{8 s} A_{\alpha \beta} \ln \left(\frac{2}{\epsilon^{1 / 2}+(\epsilon+r)^{1 / 2}}\right),
\end{aligned}
$$

where $A_{x x}=A_{y y}=1, A_{z z}=\left(s J / v_{F}\right)^{2}, A_{\alpha \neq \beta}=0$ and

$$
\kappa_{1}=\frac{2\left(v_{F} \tau\right)^{2}}{\pi^{2} \eta_{(2)}}\left[\psi^{\prime}\left(\frac{1}{2}+\frac{1}{4 \pi T \tau}\right)-\frac{3}{4 \pi T \tau} \psi^{\prime \prime}\left(\frac{1}{2}\right)\right] .
$$

In order to cut off the ultra-violet divergence in $q$ we have introduced here a cut off parameter $q_{\max }=\xi^{-1}=\eta_{(2)}^{-1 / 2}$ in complete agreement with Section 3 . Let us stress that in the framework of the phenomenological GL theory we attributed this cut off to the breakdown of the GL approach at momenta as large as $q \sim \xi^{-1}$. The microscopic approach developed here permits to see how this cut off appears: the divergent shortwave-length contribution arising from GL-like fluctuation propagators is automatically restricted by the $q$ dependencies of the impurity vertices and Green functions, which appear at the scale $q \sim l^{-1}$.

In a similar manner, the equal contributions from diagrams 7 and 8 sum to

$$
\begin{aligned}
\sigma_{\alpha \beta}^{7+8} & =-\frac{\pi e^{2}}{2 s} A_{\alpha \beta} \kappa_{2} \eta_{(2)} \int_{|\mathbf{q}| \leq q_{\max }} \frac{d^{2} \mathbf{q}}{(2 \pi)^{2}} \frac{1}{\left[\left(\epsilon+\eta_{(2)} \mathbf{q}^{2}\right)\left(\epsilon+r+\eta_{(2)} \mathbf{q}^{2}\right)\right]^{1 / 2}} \\
& \approx-\frac{e^{2} \kappa_{2}}{8 s} A_{\alpha \beta} \ln \left(\frac{2}{\epsilon^{1 / 2}+(\epsilon+r)^{1 / 2}}\right) \\
\kappa_{2}= & \frac{\left(v_{F} \tau\right)^{2}}{2 \pi^{3} T \tau} \psi^{\prime \prime}\left(\frac{1}{2}\right) .
\end{aligned}
$$


Comparing (189) and (190), we see that in the clean limit, the main contributions from the DOS fluctuations arise from diagrams 5 and 6 . In the dirty limit, diagrams 7 and 8 are also important, having $-1 / 3$ the value of diagrams 5 and 6 , for both $\sigma_{x x}$ and $\sigma_{z z}$. Diagrams 9 and 10 are not singular in $\epsilon<<1$ at all and can be neglected. The total DOS contribution to the in-plane and $c$-axis conductivity is therefore

$$
\sigma_{\alpha \beta}^{D O S}=-\frac{e^{2}}{2 s} \kappa(T \tau) A_{\alpha \beta} \ln \left(\frac{2}{\epsilon^{1 / 2}+(\epsilon+r)^{1 / 2}}\right),
$$

where

$$
\begin{aligned}
\kappa(T \tau) & =\kappa_{1}+\kappa_{2}=\frac{-\psi^{\prime}\left(\frac{1}{2}+\frac{1}{4 \pi \tau T}\right)+\frac{1}{2 \pi \tau T} \psi^{\prime \prime}\left(\frac{1}{2}\right)}{\pi^{2}\left[\psi\left(\frac{1}{2}+\frac{1}{4 \pi \tau T}\right)-\psi\left(\frac{1}{2}\right)-\frac{1}{4 \pi \tau T} \psi^{\prime}\left(\frac{1}{2}\right)\right]} \\
& \rightarrow\left\{\begin{array}{c}
56 \zeta(3) / \pi^{4} \approx 0.691, T \tau \ll 1 \\
8 \pi^{2}(T \tau)^{2} /[7 \zeta(3)] \approx 9.384(T \tau)^{2}, \quad 1 \ll T \tau \ll 1 / \sqrt{\epsilon}
\end{array}\right.
\end{aligned}
$$

is a function of $\tau T$ only. As it will be shown below at the upper limit $T \tau \sim 1 / \sqrt{\epsilon}$ the DOS contribution reaches the value of the other fluctuation contributions and in the limit of $T \tau \rightarrow \infty$ exactly eliminates the MakiThompson one.

Maki-Thompson contribution We now consider another quantum correction to fluctuation conductivity which is called the Maki-Thompson (MT) contribution (diagram 2 of Fig. 9). It was firstly discussed by Maki [6] in a paper which appeared almost simultaneously with the paper of Aslamazov and Larkin [5]. Both these articles gave rise to the microscopic theory of fluctuations in superconductor. Maki found that, in spite of the seeming weaker singularity of diagram 2 with respect to the AL one (it contains one propagator only, while the AL one contains two of them) it can contribute to conductivity comparably or even stronger than AL one.

Since the moment of its discovery the MT contribution became the subject of intense controversy. In its original paper Maki found that in $3 D$ case this fluctuation correction is four times larger than the AL one. In $2 D$ case the result was striking: the MT contribution simply diverged. This paradox was, at least at the level of recipe, resolved by Thompson [7]: he proposed to cut off the infra-red divergence in the Cooper pair center of mass momentum integration by the introduction of the finite length $l_{s}$ of inelastic scatterings of electrons on paramagnetic impurities. In the further papers of Patton 110, Keller and Korenman [11] it was cleared up that the presence of paramagnetic impurities or other external phase-breaking sources is not necessary: the fluctuation Cooper pairing of two electrons results in a change of the quasiparticle phase itself and the corresponding phase-breaking time $\tau_{\varphi}$ appears as a natural cut off parameter of the MT divergence in the strictly $2 \mathrm{D}$ case. The minimal quasi-two-dimensionality of the electron spectrum, as we will show below, automatically results in a cut off of the MT divergence. 
Although the MT contribution to the in-plane conductivity is expected to be important in the case of low pair-breaking, experiments on high-temperature superconductors have shown that the excess in-plane conductivity can usually be explained in terms of the fluctuation paraconductivity alone. Two possible explanations can be found for this fact. The first one is that the pair-breaking in these materials is not weak. The second is related with the $d$-wave symmetry of pairing which kills the anomalous Maki-Thompson process 112,113. We will consider below the case of s-pairing, where the Maki-Thompson process is well pronounced.

The appearance of the anomalously large MT contribution is nontrivial and worth being discussed. We consider the scattering lifetime $\tau$ and the pairbreaking lifetime $\tau_{\varphi}$ to be arbitrary, but satisfying $\tau_{\varphi}>\tau$. In accordance with diagram 2 of Fig.(9) the analytical expression for the MT contribution to the electromagnetic response tensor can be written as

$$
Q_{\alpha \beta}^{M T}\left(\omega_{\nu}\right)=2 e^{2} T \sum_{\Omega_{k}} \int \frac{d^{3} \mathbf{q}}{(2 \pi)^{3}} L\left(\mathbf{q}, \Omega_{k}\right) I_{\alpha \beta}\left(\mathbf{q}, \Omega_{k}, \omega_{\nu}\right),
$$

where

$$
\begin{aligned}
& I_{\alpha \beta}\left(\mathbf{q}, \Omega_{k}, \omega_{\nu}\right)=T \sum_{\varepsilon_{n}} \lambda\left(\mathbf{q}, \varepsilon_{n+\nu}, \Omega_{k-n-\nu}\right) \lambda\left(\mathbf{q}, \varepsilon_{n}, \Omega_{k-n}\right) \times \\
& \times \int \frac{d^{3} \mathbf{p}}{(2 \pi)^{3}} v_{\alpha}(\mathbf{p}) v_{\beta}(\mathbf{q}-\mathbf{p}) G\left(\mathbf{p}, \varepsilon_{n+\nu}\right) G\left(\mathbf{p}, \varepsilon_{n}\right) G\left(\mathbf{q}-\mathbf{p}, \Omega_{k-n-\nu}\right) G\left(\mathbf{q}-\mathbf{p}, \Omega_{k-n}\right) .
\end{aligned}
$$

In the vicinity of $T_{c}$, it is possible to restrict consideration to the static limit of the MT diagram, simply by setting $\Omega_{k}=0$ in (193). Although dynamic effects can be important for the longitudinal fluctuation conductivity well above $T_{L D}$, the static approximation is correct very close to $T_{c}$, as shown in [98, 125]. The main $q$-dependence in (193) arises from the propagator and vertices $\lambda$. This is why we can assume $q=0$ in Green functions and to calculate the electron momentum integral passing, as usual, to a $\xi(\mathbf{p})$ integration:

$$
\begin{aligned}
I_{\alpha \beta}\left(q, 0, \omega_{\nu}\right)= & \pi \nu\left\langle v_{\alpha}(p) v_{\beta}(q-p)\right\rangle_{F S} \times \\
& \times T \sum_{\varepsilon_{n}} \frac{1}{\left(\left|2 \varepsilon_{n+\nu}\right|+\widehat{D} q^{2}\right)} \frac{1}{\left(\left|2 \varepsilon_{n}\right|+\widehat{D} q^{2}\right)} \frac{1}{\left|\widetilde{\varepsilon}_{n+\nu}\right|+\left|\widetilde{\varepsilon}_{n}\right|} .
\end{aligned}
$$

In evaluating the sum over the Matsubara frequencies $\varepsilon_{n}$ in (195) it is useful to split it into the two parts. In the first $\varepsilon_{n}$ belongs to the domains ] $-\infty,-\omega_{\nu}[$ and $[0, \infty[$, which finally give two equal contributions. This gives rise to the regular part of the MT diagram. The second, anomalous, part of the MT diagram arises from the summation over $\varepsilon_{n}$ in the domain $\left[-\omega_{\nu}, 0[\right.$. In this interval, the further analytic continuation over $\omega_{\nu}$ leads to the appearance of an additional diffusive pole: 


$$
\begin{aligned}
I_{\alpha \beta}\left(q, 0, \omega_{\nu}\right)= & I_{\alpha \beta}^{(r e g)}\left(q, \omega_{\nu}\right)+I_{\alpha \beta}^{(a n)}\left(q, \omega_{\nu}\right)=\nu\left\langle v_{\alpha}(p) v_{\beta}(q-p)\right\rangle_{F S} \\
& \times\left[2 \pi T \sum_{n=0}^{\infty} \frac{1}{\left(2 \varepsilon_{n+\nu}+\widehat{D} q^{2}\right)} \frac{1}{\left(2 \varepsilon_{n}+\widehat{D} q^{2}\right)} \frac{1}{2 \varepsilon_{n}+\omega_{\nu}+\tau^{-1}}+\right. \\
& \left.+\frac{\pi T}{\omega_{\nu}+\tau^{-1}} \sum_{n=-\nu}^{-1} \frac{1}{\left(2 \varepsilon_{n+\nu}+\widehat{D} q^{2}\right)} \frac{1}{\left(-2 \varepsilon_{n}+\widehat{D} q^{2}\right)}\right]
\end{aligned}
$$

The limits of summation in the first sum do not depend on $\omega_{\nu}$, so it is an analytic function of this argument and can be continued to the upper halfplane of the complex frequency by the simple substitution $\omega_{\nu} \rightarrow-i \omega$. Then, tending $\omega \rightarrow 0$, one can expand the sum over powers of $\omega$ and perform the summation in terms of digamma-function:

$$
\begin{aligned}
I_{\alpha \beta}^{(r e g)}\left(q, \omega_{\nu}\right)= & \nu\left\langle v_{\alpha}(\mathbf{p}) v_{\beta}(\mathbf{q}-\mathbf{p})\right\rangle_{F S} \times \\
& \left\{\text { const }+\frac{i \omega}{2} \frac{\partial}{\partial\left(\widehat{D} q^{2}\right)}\left(\frac{\partial}{\partial\left(\widehat{D} q^{2}\right)}+\frac{\partial}{\partial\left(\tau^{-1}\right)}\right) .\right. \\
& \left.\frac{1}{\tau^{-1}-\widehat{D} q^{2}}\left[\psi\left(\frac{1}{2}+\frac{\omega_{\nu}+\widehat{D} q^{2}}{4 \pi T}\right)-\psi\left(\frac{1}{2}+\frac{\widehat{D} q^{2}}{4 \pi T}\right)\right]\right\} .
\end{aligned}
$$

The values of characteristic momenta $q \ll l^{-1}$ are determined by the domain of convergibility of the final integral of the propagator $L(\mathbf{q}, 0)$ in (193) (analogously to 190 ) and one can neglect $\widehat{D} q^{2}$ with respect to $\tau^{-1}$. In result

$$
\begin{aligned}
I_{\alpha \beta}^{(r e g) R}(q, \omega \rightarrow & 0)=\nu\left\langle v_{\alpha}(\mathbf{p}) v_{\beta}(\mathbf{q}-\mathbf{p})\right\rangle_{F S} \times \\
& \left\{\text { const }+\frac{i \omega \tau^{2}}{4}\left[\psi^{\prime}\left(\frac{1}{2}+\frac{1}{4 \pi \tau T}\right)-\psi^{\prime}\left(\frac{1}{2}\right)-\frac{\psi^{\prime \prime}\left(\frac{1}{2}\right)}{4 \pi T \tau}\right]\right\} .
\end{aligned}
$$

The appearance of the constant in $Q_{\alpha \beta}\left(\omega_{\nu}\right)$ was already discussed in the case of the AL contribution and, as was mentioned there, it is cancelled with the similar contributions of the other diagrams [98] and we will not consider it any more.

Now let us pass to the calculation of $I_{\alpha \beta}^{(a n)}\left(q, \omega_{\nu}\right)$. Expanding the summing function in simple fractions one can express the result of summation in terms of digamma-functions

$$
I_{\alpha \beta}^{(a n)}\left(q, \omega_{\nu}\right)=\frac{1}{4} \frac{\nu\left\langle v_{\alpha}(\mathbf{p}) v_{\beta}(\mathbf{q}-\mathbf{p})\right\rangle_{F S}}{\omega_{\nu}+\tau^{-1}} \frac{1}{\omega_{\nu}+\widehat{D} q^{2}} \times
$$




$$
\left[\psi\left(\frac{1}{2}+\frac{2 \omega_{\nu}+\widehat{D} q^{2}}{4 \pi T}\right)-\psi\left(\frac{1}{2}+\frac{\widehat{D} q^{2}}{4 \pi T}\right)\right] .
$$

Doing the analytical continuation $i \omega_{\nu} \rightarrow \omega \rightarrow 0$ and taking into account that in the further $q$-integration of $I_{\alpha \beta}^{(a n) R}(q, \omega \rightarrow 0)$, due to the singular at small $q$ propagator, the important range is $\widehat{D} q^{2} \ll T$, one can find

$$
I_{\alpha \beta}^{(a n) R}(q, \omega \rightarrow 0)=-\frac{i \pi \omega \tau}{16 T} \frac{\nu\left\langle v_{\alpha}(\mathbf{p}) v_{\beta}(\mathbf{q}-\mathbf{p})\right\rangle_{F S}}{-i \omega+\widehat{D} q^{2}} .
$$

Because of the considerable difference in the angular averaging of the different tensor components we discuss the MT contribution to the in-plane and out of plane conductivities separately.

Taking into account that $\left\langle v_{x}(\mathbf{p}) v_{x}(\mathbf{q}-\mathbf{p})\right\rangle_{F S}=-v_{F}^{2} / 2$ one can find that the calculation of the regular part of MT diagram to the in-plane conductivity is completely similar to the corresponding DOS contribution and here we list the final result [97] only:

$$
\sigma_{x x}^{M T(r e g)}=-\frac{e^{2}}{2 s} \tilde{\kappa} \ln \left(\frac{2}{\epsilon^{1 / 2}+(\epsilon+r)^{1 / 2}}\right),
$$

where

$$
\begin{aligned}
\tilde{\kappa}(T \tau) & =\frac{-\psi^{\prime}\left(\frac{1}{2}+\frac{1}{4 \pi \tau T}\right)+\psi^{\prime}\left(\frac{1}{2}\right)+\frac{1}{4 \pi T \tau} \psi^{\prime \prime}\left(\frac{1}{2}\right)}{\pi^{2}\left[\psi\left(\frac{1}{2}+\frac{1}{4 \pi T}\right)-\psi\left(\frac{1}{2}\right)-\frac{1}{4 \pi \tau T} \psi^{\prime}\left(\frac{1}{2}\right)\right]} \\
& \rightarrow\left\{\begin{array}{c}
28 \zeta(3) / \pi^{4} \approx 0.346, \text { for } T \tau \ll 1 \\
\pi^{2} /[14 \zeta(3)] \approx 0586, \text { for } 1 \ll T \tau \ll 1 / \sqrt{\epsilon}
\end{array}\right.
\end{aligned}
$$

is a function only of $\tau T$. We note that this regular MT term is negative, as is the overall DOS contribution.

For the anomalous part of the in-plane MT contribution we have:

$$
\begin{aligned}
\sigma_{x x}^{M T(a n)} & =8 e^{2} \eta_{(2)} T \int \frac{d^{3} q}{(2 \pi)^{3}} \frac{1}{\left[1 / \tau_{\varphi}+\hat{D} q^{2}\right]\left[\epsilon+\eta_{(2)} \mathbf{q}^{2}+\frac{r}{2}\left(1-\cos q_{z} s\right)\right]} \\
& =\frac{e^{2}}{4 s\left(\epsilon-\gamma_{\varphi}\right)} \ln \left(\frac{\epsilon^{1 / 2}+(\epsilon+r)^{1 / 2}}{\gamma_{\varphi}^{1 / 2}+\left(\gamma_{\varphi}+r\right)^{1 / 2}}\right),
\end{aligned}
$$

where, in accordance with [7], the infra-red divergence for the purely $2 \mathrm{D}$ case $(r=0)$ is cut off at $D q^{2} \sim 1 / \tau_{\varphi}{ }^{22}$. The dimensionless parameter

$$
\gamma_{\varphi}=\frac{2 \eta}{v_{F}^{2} \tau \tau_{\varphi}} \rightarrow \frac{\pi}{8 T \tau_{\varphi}}\left\{\begin{array}{c}
1, \quad T \tau \ll 1 \\
7 \zeta(3) /\left(2 \pi^{3} T \tau\right), \quad 1 \ll T \tau \ll 1 / \sqrt{\epsilon}
\end{array}\right.
$$

$\overline{22}$ The detailed study of the phase-breaking time, its energy dependence and the effect on the MT contribution was done in [115] 
is introduced for simplicity. If $r \neq 0$ the MT contribution turns out to be finite even with $\tau_{\varphi}=\infty$. Comparison of the expressions (187) and (201) indicates that in the weak pair-breaking limit, the MT diagram makes an important contribution to the longitudinal fluctuation conductivity: it is four times larger than the AL contribution in the $3 \mathrm{D}$ regime, and even logarithmically exceeds it in the $2 \mathrm{D}$ regime above $T_{L D}$. For finite pair-breaking, however, the MT contribution is greatly reduced in magnitude.

We now consider the calculation of the MT contribution to the transverse conductivity. The explicit expressions for $v_{z}(p)$ and $v_{z}(q-p)$ (see Exp. (181)), result in $\left\langle v_{x}(p) v_{x}(q-p)\right\rangle_{F S}=\frac{1}{2} J^{2} s^{2} \cos q_{z} s$. We take the limit $J \tau<<1$ in evaluating the remaining integrals, which may then be performed exactly.

The regular part of the MT contribution to the transverse conductivity is

$$
\begin{aligned}
\sigma_{z z}^{M T(\mathrm{reg})} & =-\frac{e^{2} s^{2} \pi r \tilde{\kappa}(T \tau)}{4} \int \frac{d^{3} q}{(2 \pi)^{3}} \frac{\cos q_{z} s}{\epsilon+\eta_{(2)} \mathbf{q}^{2}+\frac{r}{2}\left(1-\cos q_{z} s\right)} \\
& =-\frac{e^{2} s r \tilde{\kappa}(T \tau)}{16 \eta_{(2)}}\left(\frac{(\epsilon+r)^{1 / 2}-\epsilon^{1 / 2}}{r^{1 / 2}}\right)^{2}
\end{aligned}
$$

This term is smaller in magnitude than is the DOS one, and therefore makes a relatively small contribution to the overall fluctuation conductivity. In the $3 \mathrm{D}$ regime below $T_{L D}$, it is proportional to $J^{2}$, and in the $2 \mathrm{D}$ regime above $T_{L D}$, it is proportional to $J^{4}$.

For the anomalous part of the MT diagram one can find

$$
\begin{aligned}
\sigma_{z z}^{M T(\mathrm{an})}= & \frac{\pi e^{2} J^{2} s^{2} \tau}{4} \int \frac{d^{3} q}{(2 \pi)^{3}} \frac{\cos q_{z} s}{\left[1 / \tau_{\varphi}+\hat{\mathbf{D}} q^{2}\right]\left[\epsilon+\eta_{(2)} \mathbf{q}^{2}+\frac{r}{2}\left(1-\cos q_{z} s\right)\right]} \\
= & \frac{\pi e^{2} s}{4\left(\epsilon-\gamma_{\varphi}\right)} \int \frac{d^{2} \mathbf{q}}{(2 \pi)^{2}}\left[\frac{\gamma_{\varphi}+\eta_{(2)} \mathbf{q}^{2}+r / 2}{\left[\left(\gamma_{\varphi}+\eta_{(2)} \mathbf{q}^{2}\right)\left(\gamma_{\varphi}+\eta_{(2)} \mathbf{q}^{2}+r\right)\right]^{1 / 2}}-\right. \\
& \left.-\frac{\epsilon+\eta_{(2)} \mathbf{q}^{2}+r / 2}{\left[\left(\epsilon+\eta_{(2)} \mathbf{q}^{2}\right)\left(\epsilon+\eta_{(2)} \mathbf{q}^{2}+r\right)\right]^{1 / 2}}\right] \\
= & \frac{e^{2} s}{16 \eta_{(2)}}\left(\frac{\gamma_{\varphi}+r+\epsilon}{[\epsilon(\epsilon+r)]^{1 / 2}+\left[\gamma_{\varphi}\left(\gamma_{\varphi}+r\right)\right]^{1 / 2}}-1\right) .
\end{aligned}
$$


In examining the limiting cases of (202), it is useful to consider the cases of weak $\left(\gamma_{\varphi}<<r, \Longleftrightarrow J^{2} \tau \tau_{\varphi}>>1 / 2\right)$ and strong $\left(\gamma_{\varphi}>>r, \Longleftrightarrow J^{2} \tau \tau_{\varphi}<<\right.$ $1 / 2$ ) pair-breaking separately ${ }^{23}$. For weak pair-breaking, we have

$$
\sigma_{z z}^{M T(\text { an })} \rightarrow \frac{e^{2} s}{16 \eta_{(2)}}\left\{\begin{array}{c}
\sqrt{r / \gamma_{\varphi}}, \quad \epsilon \ll \gamma_{\varphi} \ll r \\
\sqrt{r / \epsilon}, \quad \gamma_{\varphi} \ll \epsilon \ll r \\
r /(2 \epsilon), \quad \gamma_{\varphi} \ll r \ll \epsilon
\end{array} .\right.
$$

In this case, there is the usual $3 \mathrm{D}$ to $2 \mathrm{D}$ dimensional crossover in the anomalous MT contribution at $T_{L D}$. There is an additional crossover at $T_{\varphi}$ (where $\left.T_{c}<T_{\varphi}<T_{L D}\right)$, characterized by $\epsilon\left(T_{\varphi}\right)=\gamma_{\varphi}$, below which the anomalous MT term saturates. Below $T_{L D}$, the MT contribution is proportional to $J$, but in the $2 \mathrm{D}$ regime above $T_{L D}$, it is proportional to $J^{2}$.

For strong pair-breaking

$$
\sigma_{z z}^{M T(\text { an })} \rightarrow \frac{e^{2} s}{32 \eta_{(2)}}\left\{\begin{array}{c}
r / \gamma_{\varphi}, \quad \epsilon \ll r \ll \gamma_{\varphi} \\
r^{2} /\left(4 \gamma_{\varphi} \epsilon\right), \quad r \ll \min \left\{\gamma_{\varphi}, \epsilon\right\}
\end{array} .\right.
$$

In this case, the $3 \mathrm{D}$ regime (below $T_{L D}$ ) is not singular, and the anomalous MT contribution is proportional to $J^{2}$, rather than $J$ for weak pair-breaking. In the $2 \mathrm{D}$ regime, it is proportional to $J^{4}$ for strong pair-breaking, as opposed to $J^{2}$ for weak pair-breaking. In addition, the overall magnitude of the anomalous MT contribution with strong pair-breaking is greatly reduced from that for weak pair-breaking.

Let us now compare the regular and anomalous MT contributions. Since these contributions are opposite in sign, it is important to determine which will dominate. For the in-plane resistivity, the situation is straightforward: the anomalous part always dominates over the regular and the latter can be neglected. The case of $c$-axis resistivity requires more discussion. Since we expect $\tau_{\varphi} \geq \tau$, strong pair-breaking is likely in the dirty limit. When the pair-breaking is weak, the anomalous term is always of lower order in $J$ than the regular term, so the regular term can be neglected. This is true for both the clean and dirty limits. The most important regime for the regular MT term is the dirty limit with strong pair-breaking. In this case, when $\tau_{\varphi} T \sim 1$, the regular and anomalous terms are comparable in magnitude. In short, it is usually a good approximation to neglect the regular term, except in the dirty limit with relatively strong pair-breaking and only for the out-of-plane conductivity.

Finally let us mention that the contributions from the two other diagrams of the MT type (diagrams 3 and 4 of Fig. 9) in the vicinity of critical temperature can be omitted: one can check that they have an additional square of 23

Physically the value $J^{2} \tau$. characterizes the effective interlayer tunneling rate [97.114. When $1 / \tau_{\phi}<<J^{2} \tau<<1 / \tau$, the quasiparticles scatter many times before tunneling to the neighboring layers, and the pairs live long enough for them to tunnel coherently. When $J^{2} \tau<<1 / \tau_{\phi}$, the pairs decay before both paired quasiparticles tunnel. 


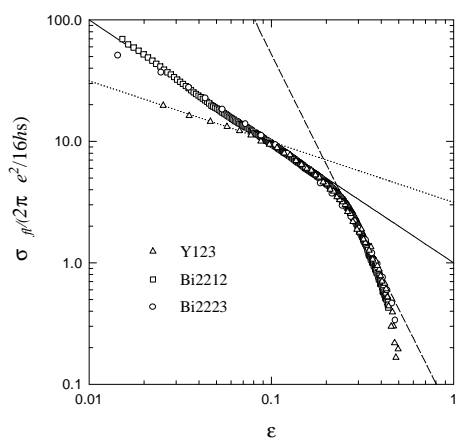

Fig. 11. The normalised excess conductivity for samples of YBCO-123 (triangles), BSSCO-2212(squares) and BSSCO-2223 (circles) plotted against $\epsilon=\ln T / T_{c}$ on a $\ln -\ln$ plot as described in [124]. The dotted and solid lines are the AL theory in 3D and $2 \mathrm{D}$ respectively. The dashed line is the extended theory of 125. .

the Cooper pair center of mass momentum $q$ in the integrand of $q$-integration with respect to diagram 2 and hence turn out to be less singular in $\epsilon$.

Discussion Although the in-plane and out-of plane components of the fluctuation conductivity tensor of a layered superconductor contain the same fluctuation contributions, their temperature behavior may be qualitatively different. In fact, for $\sigma_{x x}^{f l}$, the negative contributions are considerably less than the positive ones in the entire experimentally accessible temperature range above the transition, and it is a positive monotonic function of the temperature. Moreover, for HTS compounds, where the pair-breaking is strong and the MT contribution is in the overdamped regime, it is almost always enough to take into account only the paraconductivity to fit experimental data. Some examples of the experimental findings for in-plane fluctuation conductivity of HTS materials on can see in [116, 117, 118, 119, 120, 121, 122, 123.

In Fig. 11 the fluctuation part of in-plane conductivity $\sigma_{x x}^{f l}$ is plotted as a function of $\epsilon=\ln T / T_{c}$ on a double logarithmic scale for three HTS samples (the solid line represents the $2 D$ AL behavior $(1 / \epsilon)$, the dotted line represents the $3 D$ one: $3.2 / \sqrt{\epsilon}$ ] 124]. One can see that paraconductivity of the 
less anisotropic YBCO compound asymptotically tends to the 3D behavior $\left(1 / \epsilon^{1 / 2}\right)$ for $\epsilon<0.1$, showing the LD crossover at $\epsilon \approx 0.07$; the curve for more anisotropic 2223 phase of BSCCO starts to bend for $\epsilon<0.03$ while the most anisotropic 2212 phase of BSCCO shows a $2 D$ behavior in the whole temperature range investigated. All three compounds show a universal $2 D$ temperature behavior above the LD crossover up to the limits of the GL region. It is interesting that around $\epsilon \approx 0.24$ all the curves bend down and follow the same asymptotic $1 / \epsilon^{3}$ behavior (dashed line). Finally at the value $\epsilon \approx 0.45$ all the curves fall down indicating the end of the observable fluctuation regime.

Reggiani et al. 125] extended the $2 D$ AL theory to the high temperature region by taking into account the short wavelength fluctuations. The following universal formula for $2 D$ paraconductivity of a clean $2 \mathrm{D}$ superconductor as a function of the generalized reduced temperature $\epsilon=\ln T / T_{c}$ was obtained ${ }^{24}$ :

$$
\sigma_{x x}^{f l}=\frac{e^{2}}{16 s} f(\epsilon)
$$

with $f(\epsilon)=\epsilon^{-1}, \epsilon \ll 1$ and $f(\epsilon)=\epsilon^{-3}, \epsilon \gtrsim 1$.

In the case of the out-of-plane conductivity the situation is quite different. Both positive contributions (AL and anomalous MT) are suppressed by the interlayer transparency, leading to a competition between positive and negative terms. This can lead to a maximum in the c-axis fluctuation resistivity which occurs in the $2 \mathrm{D}$ regime (in the case discussed $J \tau<<1, r \kappa<<1$ and $\left.\gamma_{\varphi} \kappa>1\right)$ :

$$
\epsilon_{m} / r \approx \frac{1}{(8 r \kappa)^{1 / 2}}-\frac{1}{8 \kappa}\left[\tilde{\kappa}-\frac{1}{2 \gamma_{\varphi}}\right]
$$

This nontrivial effect of fluctuations on the transverse resistance of a layered superconductor allows a successful fit to the data observed on optimally and overdoped HTS samples (see, for instance, Fig.12) where the growth of the resistance still can be treated as the correction.

The fluctuation mechanism of the growth of the transverse resistance can be easily understood in a qualitative manner. Indeed to modify the in-plane result (100) for the case of c-axis paraconductivity one has to take into account the hopping character of the electronic motion in this direction. If the probability of one-electron interlayer hopping is $\mathcal{P}_{1}$, then the probability of coherent hopping for two electrons during the fluctuation Cooper pair lifetime $\tau_{G L}$ is the conditional probability of these two events: $\mathcal{P}_{2}=\mathcal{P}_{1}\left(\mathcal{P}_{1} \tau_{G L}\right)$. The transverse paraconductivity may thus be estimated as $\sigma_{\perp}^{A L} \sim \mathcal{P}_{2} \sigma_{\|}^{A L} \sim \mathcal{P}_{1}^{2} \frac{1}{\epsilon^{2}}$, in complete accordance with (188). We see that the temperature singularity of $\sigma_{\perp}^{A L}$ turns out to be stronger than that in $\sigma_{\|}^{A L}$, however for a strongly

$\overline{{ }^{24} \text { In Section } 7.2}$ we will demonstrate how such a dependence $\left(1 / \ln ^{3}\left(T / T_{\mathrm{c}}\right)\right)$ appears by accounting for short wavelength fluctuations for the $2 D$ fluctuation susceptibility. 


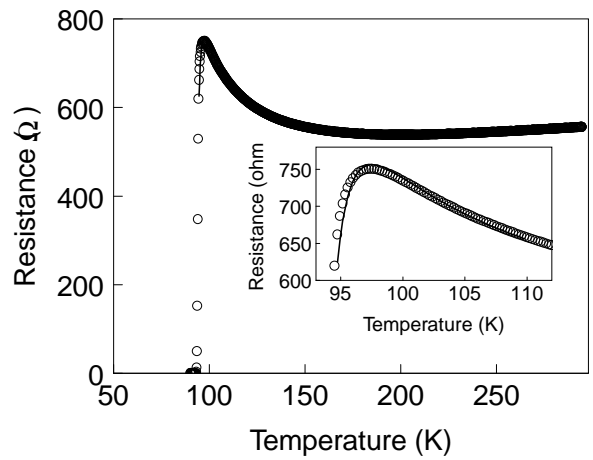

Fig. 12. Fit of the temperature dependence of the transverse resistance of an underdoped BSCCO c-axis oriented film with the results of the fluctuation theory 126. The inset shows the details of the fit in the temperature range between $T_{c}$ and $110 \mathrm{~K}$.

anisotropic layered superconductor $\sigma_{\perp}^{A L}$ is considerably suppressed by the square of the small probability of inter-plane electron hopping which enters in the pre-factor. It is this suppression which leads to the necessity of taking into account the DOS contribution to the transverse conductivity. The latter is less singular in temperature but, in contrast to the paraconductivity, manifests itself in the first, not the second, order in the interlayer transparency $\sigma_{\perp}^{D O S} \sim-\mathcal{P}_{1} \ln \frac{1}{\varepsilon}$. The DOS fluctuation correction to the one-electron transverse conductivity is negative and, being proportional to the first order of $\mathcal{P}_{1}$, can completely change the traditional picture of fluctuations just rounding the resistivity temperature dependence around transition. The shape of the temperature dependence of the transverse resistance mainly is determined by competition of the opposite sign contributions: the paraconductivity and MT term, which are strongly temperature dependent but are suppressed by the square of the barrier transparency and the DOS contribution which has a weaker temperature dependence but depends only linearly on the barrier transparency.

\section{Manifestation of fluctuations in various properties}

In this Section we will demonstrate the applications of the microscopic theory of fluctuations. The limited volume does not permit us to deliver here the systematic review of the modern theory and we restrict ourselves only by presentation of the several representative recent studies. The details one can find in the articles cited in subtitles.

It is necessary to underline that the comparison of the results of fluctuation theory with the experimental findings on HTS materials has to be 
considered sooner in qualitative than quantitative context. Indeed, as is clear now, the superconductivity in the most of HTS compounds has the nontrivial symmetry. Moreover, as was discussed in the previous Section, these compounds are rather clean than dirty. Both these complications can be taken into account (see for example [112,97]) but this was not done in the majority of the cited papers.

\subsection{The effects of fluctuations on magnetoconductivity 97, 127, 154, 155}

The experimental investigations of the fluctuation magnetoconductivity are of special interest first because this physical value weakly depends on the normal state properties of superconductor and second due to its special sensitivity to temperature and magnetic field. The role of AL contribution for both the in-plane and out-of-plane magnetoconductivities was studied above in the framework of the phenomenological approach. The microscopic calculations of the other fluctuation corrections to the in-plane magnetoconductivity conductivity show that the MT contribution has the same positive sign and temperature singularity as the $\mathrm{AL}$ one. In the case of weak pair-breaking it can even considerably exceed the latter. The negative DOS contribution, like in the case of the zero-field conductivity, turns out to be considerably less singular and many authors (see e.g. Refs. 136, 137, 138, 139, 142, 140,141, 143, 144, 145, 146, 147) successfully explained the in-plane magnetoresistance data in HTS using the $\mathrm{AL}$ and MT contributions only 148, 149, 150,151.

Turning to the out-of-plane magnetoconductivity of a layered superconductor one can find a quite different situation. Both the AL and MT contributions turn out to be here of the second order in the interlayer transparency and this circumstance makes the less singular DOS contribution, which remains however of first order in transparency, to be competitive with the main terms 127]. The large number of microscopic characteristics involved in this competition, like the Fermi velocity, interlayer transparency, phase-breaking and elastic relaxation times, gives rise to the possibility of occurrence of different scenarios for various compounds. The c-axis magnetoresistance of a set of HTS materials shows a very characteristic behavior above $T_{c 0}$. In contrast to the ab-plane magnetoresistance which is positive at all temperatures, the magnetoresistance along the c-axis has been found in many HTS compounds (BSSCO 128, 130, 131, 132, LSSCO [133, YBCO [134 and TIBCCO 1135]) to have a negative sign not too close to $T_{c 0}$ and turn positive at lower temperatures. We will show how this behavior find its explanation within the fluctuation theory 97.

We consider here the effect of a magnetic field parallel to the $c$-axis. In this case both quasiparticles and Cooper pairs move along Landau orbits within the layers. The $c$-axis dispersion remains unchanged from the zero-field form. In the chosen geometry one can generalize the zero-field results reported in 
the previous Section to finite field strengths simply by the replacement of the two-dimensional integration over $\mathbf{q}$ by a summation over the Landau levels

$$
\int \frac{d^{2} \mathbf{q}}{(2 \pi)^{2}} \rightarrow \frac{H}{\Phi_{0}} \sum_{n}=\frac{h}{2 \pi \eta_{(2)}} \sum_{n}
$$

(let us remind that $\eta_{(2)}=\xi_{x y}^{2}$ ). So the general expressions for all fluctuation corrections to the c-axis conductivity in a magnetic field can be simply written in the form [97]:

$$
\begin{aligned}
& \sigma_{z z}^{A L}=\frac{e^{2} s r^{2} h}{64 \xi_{x y}^{2}} \sum_{n=0}^{\infty} \frac{1}{\{[\epsilon+h(2 n+1)][r+\epsilon+h(2 n+1)]\}^{3 / 2}} \\
& \sigma_{z z}^{D O S}=-\frac{e^{2} s r \kappa h}{8 \xi_{x y}^{2}} \sum_{n=0}^{1 / h} \frac{1}{\{[\epsilon+h(2 n+1)][r+\epsilon+h(2 n+1)]\}^{1 / 2}} \\
& \sigma_{z z}^{M T(r e g)}=-\frac{e^{2} s \tilde{\kappa} h}{4 \xi_{x y}^{2}} \sum_{n=0}^{\infty}\left(\frac{\epsilon+h(2 n+1)+r / 2}{\{[\epsilon+h(2 n+1)][r+\epsilon+h(2 n+1)]\}^{1 / 2}}-1\right) \\
& \sigma_{z z}^{M T(a n)}=\frac{e^{2} s h}{8 \xi_{x y}^{2}\left(\varepsilon-\gamma_{\varphi}\right)} \sum_{n=0}^{\infty}\left(\frac{\gamma_{\varphi}+h(2 n+1)+r / 2}{\left\{\left[\left(\gamma_{\varphi}+h(2 n+1)\right]\left[\gamma_{\varphi}+h(2 n+1)+r\right)\right]\right\}^{1 / 2}}-\right. \\
& \left.-\frac{\epsilon+h(2 n+1)+r / 2}{\{[\epsilon+h(2 n+1)][r+\epsilon+h(2 n+1)]\}^{1 / 2}}\right)
\end{aligned}
$$

For the in-plane component of the fluctuation conductivity tensor the only additional problem appears in the AL diagram, where the matrix elements of the harmonic oscillator type, originating from the $B_{\|}\left(q_{\|}\right)$blocks, have to be calculated. How to do this was demonstrated in details in Section 4. The other contributions are essentially analogous to their c-axis counterparts:

$$
\begin{aligned}
& \sigma_{x x}^{A L}= \frac{e^{2}}{4 s} \sum_{n=0}^{\infty}(n+1)\left(\frac{1}{\{[\epsilon+h(2 n+1)][r+\epsilon+h(2 n+1)]\}^{1 / 2}}-\right. \\
& \frac{2}{\{[\epsilon+h(2 n+2)][r+\epsilon+h(2 n+2)]\}^{1 / 2}}+ \\
&\left.\frac{1}{\{[\epsilon+h(2 n+3)][r+\epsilon+h(2 n+3)]\}^{1 / 2}}\right), \\
& \sigma_{x x}^{D O S}+\sigma_{x x}^{M T(r e g)}=-\frac{e^{2} h(\kappa+\tilde{\kappa})}{2 s} \sum_{n=0}^{1 / h} \frac{1}{\{[\epsilon+h(2 n+1)][r+\epsilon+h(2 n+1)]\}^{1 / 2}}
\end{aligned}
$$


and

$$
\begin{aligned}
\sigma_{x x}^{M T(a n)=} & \frac{e^{2} h}{4 s\left(\epsilon-\gamma_{\varphi}\right)} \sum_{n=0}^{\infty}\left(\frac{1}{\left\{\left[\left(\gamma_{\varphi}+h(2 n+1)\right]\left[\gamma_{\varphi}+h(2 n+1)+r\right)\right]\right\}^{1 / 2}}-\right. \\
& \left.\frac{1}{\{[\epsilon+h(2 n+1)][r+\epsilon+h(2 n+1)]\}^{1 / 2}}\right) .
\end{aligned}
$$

These results can in principle be already used for numerical evaluations and fitting of the experimental data which was indeed successfully done in a series of papers 134,152,135.

The detailed comparison of the cited results with the experimental data 134 153, especially in strong fields, raised the problem of regularization of the DOS contribution. If in the absence of the magnetic field its ultra-violet divergence was successfully cut off at $q \sim \xi^{-1}$, in the case under consideration the cut off parameter depends on the magnetic field and makes the fitting procedure ambiguous. The solution of this problem was proposed in [154, where the authors calculated the difference $\Delta \sigma_{z z}^{D O S}=\sigma_{z z}^{D O S}(h, \epsilon)-\sigma_{z z}^{D O S}(0, \epsilon)$ applying to formulas (204) and (208) the same trick which was already used in Section 2 for the regularization of the free energy in magnetic field (Eq. (78)). The corresponding asymptotics for all out-of-plane fluctuation contributions are presented in the following table:

\begin{tabular}{|l|l|l|l|}
\hline & $h \ll \epsilon$ & $\epsilon \ll h \ll r$ & $\max \{\epsilon, r\} \ll h \quad(2 D)$ \\
\hline$\Delta \sigma_{z z}^{D O S}$ & $\frac{e^{2} s \kappa}{32^{5} \xi_{x y}^{2}} \frac{r(\epsilon+r / 2)}{[\epsilon(\epsilon+r)]^{3 / 2}} h^{2}$ & $0.428 \frac{e^{2} s \kappa}{16 \xi_{x y}^{2}} r \sqrt{\frac{h}{r}}$ & $\frac{e^{2} s \kappa}{8 \xi_{x y}^{2}} r \ln \frac{\sqrt{h}}{\sqrt{\epsilon}+\sqrt{\epsilon+r}}$ \\
\hline$\Delta \sigma_{z z}^{M T(r e g)}$ & $\frac{e^{2} s \tilde{\kappa}}{32^{6} \xi_{x y}^{2}} \frac{r^{2}}{[\epsilon(\epsilon+r)]^{3 / 2}} h^{2}$ & $0.428 \frac{e^{2} s \tilde{\kappa}}{8 \xi_{x y}^{2}} r \sqrt{\frac{h}{r}}$ & $-\sigma_{z z}^{M T(r e g)}(0, \epsilon)-\frac{\pi^{2} e^{2} s \tilde{\kappa}}{2^{8} \xi_{x y}^{2}} \frac{r^{2}}{h}$ \\
\hline$-\Delta \sigma_{z z}^{A L}$ & $\frac{e^{2} s}{2^{8} \xi_{x y}^{2}} \frac{r^{2}(\epsilon+r / 2)}{[\epsilon(\epsilon+r)]^{5 / 2}} h^{2}$ & $\sigma_{z z}^{A L}(0, \epsilon)-\frac{3.24 e^{2} s}{\xi_{x y}^{2}} \sqrt{\frac{r}{h}}$ & $\sigma_{z z}^{A L}(0, \epsilon)-\frac{7 \zeta(3) e^{2} s}{2^{9} \xi_{x y}^{2}} \frac{r^{2}}{h^{2}}$ \\
\hline $\begin{array}{c}-\Delta \sigma_{z z}^{M T(a n)} \\
\min \{\epsilon, r\} \ll \gamma_{\varphi}\end{array}$ & $\frac{e^{2} s}{32^{7} \xi_{x y}^{2}} \frac{r^{2}}{[\epsilon(\epsilon+r)]^{2}} h^{2}$ & $\sigma_{z z}^{M T(a n)}(0, \epsilon)-\frac{e^{2} s}{32 \xi_{x y}^{2}} \sqrt{\frac{r}{\gamma_{\varphi}}}$ & $\sigma_{z z}^{M T(a n)}(0, \epsilon)-\frac{3 \pi^{2} e^{2} s}{2^{8} \xi_{x y}^{2}} \frac{\max \left\{r, \gamma_{\varphi}\right\}}{h}$ \\
\hline $\begin{array}{c}-\Delta \sigma_{z z}^{M T(a n)} \\
\gamma_{\varphi} \ll \min \{\epsilon, r\}\end{array}$ & $\frac{e^{2} s}{32^{7} \xi_{x y}^{2}} \frac{\sqrt{r}}{\epsilon \gamma_{\varphi}^{3 / 2}} h^{2}$ & $\sigma_{z z}^{M T(a n)}(0)-\frac{3.24 e^{2} s}{64 \xi_{x y}^{2}} \sqrt{\frac{r}{h}}$ & $\sigma_{z z}^{M T(a n)}(0, \epsilon)-\frac{3 \pi^{2} e^{2} s}{2^{8} \xi_{x y}^{2}} \frac{(r+\epsilon)}{h}$ \\
\hline
\end{tabular}

Table 3

The procedure described gives an excellent fitting up to very high fields [155 which is shown in Fig . 13.

Let us start the analysis from the $2 D$ case $(r \ll \epsilon)$. One can see that here the positive DOS contribution to magnetoconductivity turns out to be dominant. It grows as $H^{2}$ up to $H_{c 2}(\epsilon)$ and then crosses to a slow logarithmic asymptote. At $H \sim H_{c 2}(0)$ the value of $\Delta \sigma_{z z}^{D O S}(h \sim 1, \epsilon)=-\sigma_{z z}^{D O S}(0, \epsilon)$ which means the total suppression of the fluctuation correction in such a strong field. The regular part of the Maki-Thompson contribution does not manifest itself in this case while the AL term can compete with the DOS one in the immediate vicinity of $T_{c}$, where the small anisotropy factor $r$ can be compensated by the additional $\epsilon^{3}$ in the denominator. The anomalous MT 


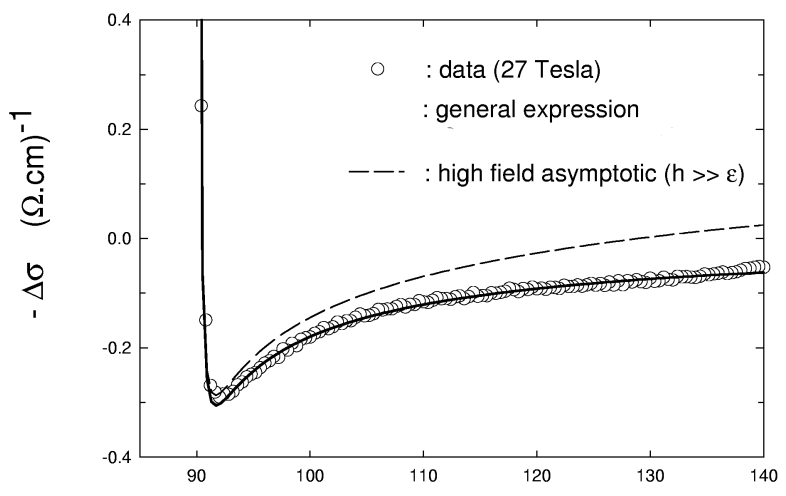

Fig. 13. Magnetoconductivity versus temperature at $27 \mathrm{~T}$ for an underdoped Bi2212 single crystal. The solid line represents the theoretical calculation. The symbols are the experimental magnetoconductivity $\Delta \sigma_{z z}(B\|c\| I) 155$

contribution can contribute in the case of small pairbreaking only, which is opposite to what is expected in HTS.

In the $3 D$ case $(\epsilon \ll r)$ the behavior of the magnetoconductivity is more complex. In weak and intermediate fields the main, negative, contribution to the magnetoconductivity occurs from the AL and MT terms. At $H \sim$ $H_{c 2}(\epsilon)(h \sim \epsilon)$ the paraconductivity is already considerably suppressed by the magnetic field and the $h^{2}$ - dependence of the magnetoconductivity changes through the $\sqrt{\frac{r}{h}}$ tendency to the high field asymptote $-\sigma_{z z}^{(f l)}(0, \epsilon)$. In this intermediate region of fields $(\epsilon \ll h \ll r)$, side by side with the decrease $(\sim$ $\sqrt{\frac{r}{h}}$ ) of the main AL and MT contributions, the growth of the still relatively small DOS term takes place. At the upper limit of this region $(h \sim r)$ its positive contribution is of the same order as the AL one and at high fields $(r \ll h \ll 1)$ the DOS contribution determines the slow logarithmic decay of the fluctuation correction to the conductivity which is completely suppressed only at $H \sim H_{c 2}(0)$. The regular part of the Maki- Thompson contribution is not of special importance in the $3 D$ case. It remains comparable with the DOS contribution in the dirty case at fields $h \lesssim r$, but decreases rapidly $\left(\sim \frac{r}{h}\right)$ at strong fields $(h \gtrsim r)$, in the only region where the robust $\Delta \sigma_{c}^{D O S}(h, \epsilon) \sim \ln \frac{h}{r}$ shows up surviving up to $h \sim 1$.

The temperature dependence of the different fluctuation contributions to the magnetoconductivity calculated for an underdoped Bi-2212 single crystal at the magnetic field $27 \mathrm{~T}$ is presented in Fig. 14.

The formulas for the in-plane magnetoconductivity are presented in the table: 


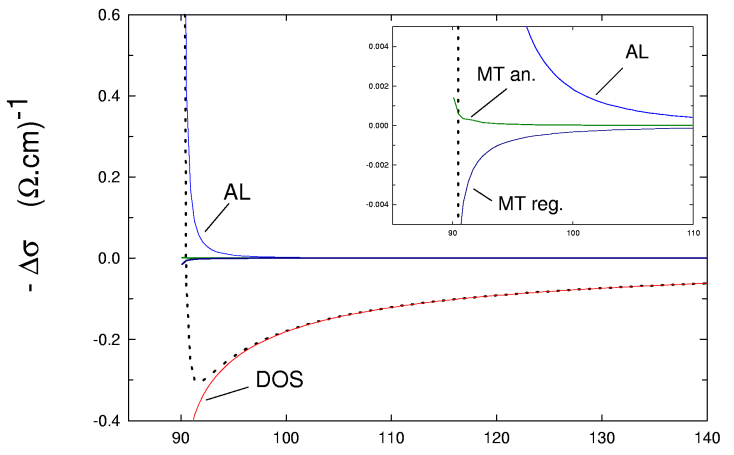

Fig. 14. Decomposition of the calculation of total theoretical magnetoconductivity for an underdoped Bi-2212 single crystal at $27 \mathrm{~T}$. The inset shows the regular and anomalous parts of the MT contribution which are too small to be presented in the same scale as the AL and DOS contributions [155].

\begin{tabular}{|c|c|c|}
\hline & $h \ll \epsilon$ & $\epsilon \ll h \ll r ; \max \{\epsilon, r\} \ll h$ \\
\hline$\Delta \sigma_{x x}^{A L}$ & $-\frac{e^{2}}{2^{8} s} \frac{\left[8 \epsilon(\epsilon+r)+3 r^{2}\right]}{[\epsilon(\epsilon+r)]^{5 / 2}} h^{2}$ & $-\sigma_{x x}^{A L}(0, \epsilon)+\frac{e^{2}}{4 s} \frac{1}{\sqrt{2 h r}} ;-\sigma_{x x}^{A L}(0, \epsilon)+\frac{e^{2}}{8 s} \frac{1}{h}$ \\
\hline $\begin{array}{c}\Delta \sigma_{x x}^{M T(a n)} \\
\left(\min \{\epsilon, r\} \ll \gamma_{\varphi}\right)\end{array}$ & $-\frac{e^{2}}{32^{5} s} \frac{(\epsilon+r / 2)}{[\epsilon(\epsilon+r)]^{3 / 2}} h^{2}$ & $-\sigma_{x x}^{M T}(0, \epsilon)+\frac{e^{2}}{8 s} \frac{1}{\gamma_{\varphi}} \ln \frac{\sqrt{\gamma_{\varphi}}}{\sqrt{2 h}+\overline{\bar{v}} 2 h+r}$ \\
\hline $\begin{array}{c}\Delta \sigma_{x x}^{M T(a n)} \\
\left(\gamma_{\varphi} \ll \min \{\epsilon, r\}\right)\end{array}$ & $-\frac{e^{2}}{32^{5} s} \frac{1}{\epsilon \gamma^{3 / 2} r^{1 / 2}} h^{2}$ & $-\sigma_{x x}^{M T}(0, \epsilon)+$ \\
\hline $\begin{array}{l}\Delta\left(\sigma_{x x}^{D O S}+\right. \\
\left.\sigma_{x x}^{M T(r e g)}\right)\end{array}$ & $\frac{e^{2}(\kappa+\tilde{\kappa})}{32^{7} s} \frac{(\varepsilon+r / 2)}{[\varepsilon(\varepsilon+r)]^{3 / 2}} h^{2}$ & $\frac{e^{2}(\kappa+\tilde{\kappa})}{32 s} \ln \frac{\sqrt{h}}{(\sqrt{\epsilon}+\sqrt{\epsilon+r})}$ \\
\hline
\end{tabular}

Table 4

Analyzing it one can see that in almost all regions the negative AL and MT contributions govern the behavior of in-plane magnetoconductivity. Nevertheless, similar to the c-axis case, the high field behavior is again determined by the positive logarithmic $\Delta\left(\sigma_{x x}^{D O S}+\sigma_{x x}^{M T(r e g)}\right)$ contribution, which is the only one to survive in strong field. It is important to stress that the suppression of the DOS contribution by a magnetic field takes place very slowly. Such robustness with respect to the magnetic field is of the same physical origin as the slow logarithmic dependence of the DOS-type corrections on temperature.

Another important problem which appears in the fitting of the resistive transition shape in relatively strong fields with the fluctuation theory is the much larger broadening of the transition than predicted by the AbrikosovGorkov theory [156]. Kim and Gray [96] explained the broadening of the c-axis peak with increasing magnetic field in terms of Josephson coupling, describing a layered superconductor as a stack of Josephson junctions. In Refs. 
157.71 the self-consistent Hartree approach was proposed for the extension of fluctuation theory beyond the Gaussian approximation. It results in the considerable shift of $T_{c}(H)$ toward low temperatures with a corresponding broadening of the transition. The renormalized reduced temperature $\tilde{\varepsilon}_{h}$ is determined according to the self-consistent equation [71]:

$$
\varepsilon_{h}=\tilde{\varepsilon}_{h}-\frac{1}{4} G i_{(2)} h \sum_{n=0}^{1 / h} \frac{1}{\left[\left(\tilde{\varepsilon}_{h}+h n\right)\left(\tilde{\varepsilon}_{h}+h(n+1)+r\right)\right]^{1 / 2}}
$$

The authors of 158 , following the procedure proposed by Dorsey and Ullah [71, modified the expressions (203)- (209) by account for 210). In result they succeeded to fit quantitatively both in-plane resistivity transition and the transverse resistivity peak for BSCCO films strongly broadened by applied magnetic field.

\subsection{Fluctuations far from $T_{c}$ or in strong magnetic fields}

As was mentioned above the role of fluctuations is especially pronounced in the vicinity of the critical temperature. Nevertheless for some phenomena they can be still considerable far from the transition too. In these cases the GL theory is certainly unapplicable since the short-wave and dynamical fluctuation contributions have to be taken into account. It can be done in the microscopic approach which we will demonstrate in several examples.

Fluctuation magnetic susceptibility far from transition [29]. The given above qualitative estimations (58)-(64) for the fluctuation diamagnetic susceptibility, based on the Langevin formula, demonstrate that even at high temperatures $T \gg T_{c}$ it turns to be of the order of $\chi_{P}$ for clean $3 D$ superconductors and exceeds noticeably this value for $2 D$ systems. In order to develop the microscopic theory [159,29, 161 let us start from the general expression for free energy in the one-loop approximation:

$$
F=T \sum_{\Omega_{k}} \operatorname{Tr}\left\{\ln \left[1-g \Pi\left(\Omega_{k}, \mathbf{r}, \mathbf{r}^{\prime}\right)\right]\right\},
$$

where $g$ is the effective interaction constant related with the transition critical temperature by (158). This approximation corresponds to the ladder one (see (150)) for the fluctuation propagator. The polarization operator $\Pi\left(\Omega_{k}, \mathbf{r}, \mathbf{r}^{\prime}\right)$ is determined by expression (151) but in the case of an applied magnetic field the homogeneity of the system is lost and $\Pi\left(\Omega_{k}, \mathbf{r}, \mathbf{r}^{\prime}\right)$ depends not on the space variable difference $\mathbf{r}-\mathbf{r}^{\prime}$ but on each separately. Expanding $\Pi\left(\Omega_{k}, \mathbf{r}, \mathbf{r}^{\prime}\right)$ one can express the magnetic susceptibility of a layered superconductor in a weak magnetic field perpendicular to the layers in terms of the derivatives $\Pi_{x}=\frac{\partial}{\partial q_{x}} \Pi(\mathbf{q})$ 29:

$$
\chi=-\frac{\partial^{2} F}{\partial H^{2}}=-\frac{2}{3} e^{2} T \sum_{\Omega_{k}} \int \frac{d^{3} \mathbf{q}}{(2 \pi)^{3}} L^{3} \Pi_{x}\left(\Pi_{x} \Pi_{y y}-\Pi_{y} \Pi_{x y}\right) .
$$


The final expressions for the fluctuation diamagnetic susceptibility in the clean and dirty cases for wide range of temperatures can be written as:

$$
\begin{aligned}
& \chi_{f l}^{(3)}(T)=\frac{\chi_{P}}{3}\left\{\begin{array}{c}
\left.0.05\left(\ln ^{-2}\left(\omega_{D} / T_{c}\right)\right)-\ln ^{-2}\left(T / T_{c}\right)\right), \tau^{-1} \ll T \ll \omega_{D} \\
2 \sqrt{T \tau} \ln ^{-1}\left(T / T_{c}\right), T_{c} \ll T \ll \tau^{-1}
\end{array}\right. \\
& \chi_{f l}^{(2)}(T)=\frac{0.05}{p_{F} s} \chi_{P}\left(\frac{E_{F}}{T}\right) \frac{1}{\ln ^{3}\left(T / T_{c}\right)}
\end{aligned}
$$

Let us stress that these results are valid for the fluctuation diamagnetism of a normal metal with $g>0$ too, if by $T_{c}$ one uses the formal value $T_{c} \sim$ $E_{F} \exp \left(\frac{1}{\nu g}\right)$.

Fluctuation magnetoconductivity far from transition 162. Let us discuss the conductivity of the $2 D$ electron system with impurities in a magnetic field at low temperatures. Even in the absence of the field the effects of quantum interference of the non-interacting electrons in their scatterings on elastic impurities already results in the appearance of a nontrivial temperature dependence of the resistance. This result contradicts the statement of the classical theory of metals requiring the saturation of the resistance at its residual value at low temperatures. In a superconductor above the critical temperature this, so-called weak localization (WL), effect is amplified by the Andreev reflection of electrons on the fluctuation Cooper pair leading to appearance of the MT correction to the conductivity. The characteristic feature of both the MT and WL corrections is their extreme sensitivity to the dephasing time $\tau_{\varphi}$ and to weak magnetic fields.

Beyond the GL region $\left(T \gtrsim T_{c}\right)$ the MT correction is determined by the same diagram 2 of Fig. 9 but now the dynamic $\left(\Omega_{k} \neq 0\right)$ and shortwave-length $\left(q \sim \xi^{-1}\right)$ fluctuation modes have to be taken into account. The corresponding calculations were performed in 98, 162 and the result can be written in the form:

$$
\delta \sigma_{W L+M T}=\frac{e^{2}}{2 \pi^{2}}[\alpha-\beta(T)] Y\left(\Omega_{L} \tau \epsilon\right),
$$

where we introduced the effective Larmour frequency for the diffusion motion $\Omega_{L}=4 \mathcal{D} e H$ with the diffusion coefficient $\mathcal{D} 25$ and the function

$$
Y(x)=\ln x+\psi\left(\frac{1}{2}+\frac{1}{x}\right)=\left\{\begin{array}{c}
\frac{x^{2}}{24}, x \ll 1 \\
\ln x, x \gg 1
\end{array} .\right.
$$

The first term in this formula corresponds to the WL contribution $(\alpha=1$ if the spin-orbit interaction of the electrons with the impurities is small while

$\overline{{ }^{25} \text { Comparison }}$ of the expressions (77), (117) and (215) relates the Larmour frequency with the dimensionless field: $h=\Omega_{L} / 2 T_{c}$ introduced in section 2 and the diffusion coefficient with the phenomenological GL constants $\mathcal{D}=1 / m \alpha$. 
in the opposite limiting case $\alpha=-1 / 2$ ), the second describes the MT contribution to magnetoconductivity. The function $\beta\left[\ln \left(T / T_{c}\right)\right]$ was introduced in Ref. 162. At $T \rightarrow T_{c} \beta(x)=1 / x$ and (215) reduces to the already studied MT correction in the vicinity of critical temperature. For $T \gg T_{c} \beta(x)=1 / x^{2}$ and the MT contribution gives a logarithmically small correction to the WL result. Its zero-field value, being proportional to $\ln ^{-2}\left(T / T_{c}\right)$, decreases with the growth of the temperature faster than both the AL contribution (in the dirty case $\delta \sigma_{A L} \sim 1 / \ln \left(T / T_{c}\right)$ ) and the especially slow DOS contribution $\left(\delta \sigma_{D O S} \sim \ln \ln \left(1 / T_{c} \tau\right)-\ln \ln \left(T / T_{c}\right)\right)$ (see Ref. 98.99).

It worth mentioning that for the region of temperatures $T \gg T_{c}$, analogous to Exp.(213)- (214), the result (215) can be applied both to superconducting and normal metals $(g>0)$, if in place of the critical temperature the formal value $T_{c} \sim E_{F} \exp \left(\frac{1}{\nu g}\right)$ is undermined. The interplay of the localization and fluctuation corrections was extensively studied (see, for example, (163, 164, 165, 166, 167)

Fluctuations in magnetic fields near $\boldsymbol{H}_{c 2}(0)$ [168]. As one can see from (207)- (209), in the vicinity of the upper critical field $H_{c 2}(T)$ the fluctuation corrections diverge as $\epsilon_{h}^{-1}$ for the $2 D$ case and as $\epsilon_{h}^{-1 / 2}$ for the $3 D$ case 2 (it is enough to keep just the terms with $n=0$ in these formulas). This behavior is preserved in strong magnetic fields too, but the coefficients undergo changes. A case of special interest is $T \ll T_{c}$ (which means $H \rightarrow H_{c 2}(0)$ ) which represents an example of a quantum phase transition 168. Microscopic analysis of the magnetoconductivity permits us to study the effect of fluctuations in magnetic fields of the order of $H_{c 2}(0)$, where the GL functional approach is inapplicable.

We restrict our consideration to the case of a dirty metal $(T \tau \ll 1)$. In this limit $\left|\tilde{\omega}_{n+\mu}-\tilde{\omega}_{-n}\right| \approx \tau^{-1}$ and the Green function correlator (153) can be written in the form

$$
\mathcal{P}\left(\mathbf{q}, \varepsilon_{1}, \varepsilon_{2}\right)=2 \pi \nu \tau^{2} \theta\left(-\varepsilon_{1} \varepsilon_{2}\right)\left(\tau^{-1}-\left|\varepsilon_{1}-\varepsilon_{2}\right|-\widehat{\mathcal{D}} q^{2}\right) .
$$

Expressing $\Pi\left(\mathbf{q}, \Omega_{k}\right)$ in terms of $\mathcal{P}\left(\mathbf{q}, \varepsilon_{1}, \varepsilon_{2}\right)$ by means of $(172)$ and using the definition of the critical temperature one can find an explicit formula for the fluctuation propagator

$$
\begin{aligned}
L^{-1}\left(\mathbf{q}, \Omega_{k}\right) & =g^{-1}-\Pi\left(\mathbf{q}, \Omega_{k}\right)= \\
& =-\nu\left[\ln \frac{T}{T_{c}}+\psi\left(\frac{1}{2}+\frac{\left|\Omega_{k}\right|+\widehat{\mathcal{D}} \mathbf{q}^{2}}{4 \pi T}\right)-\psi\left(\frac{1}{2}\right)\right] .
\end{aligned}
$$

The prominent characteristic of this expression is that it is valid even relatively far from the critical temperature (for temperatures $T \ll \min \left\{\tau^{-1}, \omega_{D}\right\}$ ) and for $|\mathbf{q}| \ll l^{-1},\left|\Omega_{k}\right| \ll \omega_{D}$.

${ }^{26} \epsilon_{h}$ is the renormalized by the magnetic field reduced temperature $\epsilon_{h}=\epsilon+h$ 
One can rewrite this expression in a magnetic field applied along the c-axis in the Landau representation by simply replacing $\left(\widehat{\mathcal{D}} \mathbf{q}^{2}\right)_{\|} \Rightarrow \Omega_{L}(n+1 / 2)$ [159:

$$
\begin{aligned}
L_{n}^{-1}\left(q_{z}, \Omega_{k}\right)= & -\nu\left[\ln \frac{T}{T_{c}}+\psi\left(\frac{1}{2}+\frac{\left|\Omega_{k}\right|}{4 \pi T}+\right.\right. \\
& \left.\left.+\frac{\Omega_{L}(n+1 / 2)+4 \tau J^{2} \sin ^{2}\left(q_{z} s / 2\right)}{4 \pi T}\right)-\psi\left(\frac{1}{2}\right)\right] .
\end{aligned}
$$

In the case of arbitrary temperatures and magnetic fields the expression for the AL contribution to the conductivity takes the form:

$$
\begin{aligned}
\sigma_{x x}^{A L}\left(\omega_{\nu}\right)= & \nu e^{2} T \sum_{\Omega_{k}} \sum_{n, m=0}^{\infty} B_{n, m}\left(\Omega_{k}+\omega_{\nu}, \Omega_{k}\right) L_{m}\left(\Omega_{k}\right) \times \\
& \times B_{m, n}\left(\Omega_{k}, \Omega_{k}+\omega_{\nu}\right) L_{n}\left(\Omega_{k}+\omega_{\nu}\right)
\end{aligned}
$$

(we have restricted our consideration to the $2 D$ case). The expression for $B_{n, m}\left(\Omega_{k}, \omega_{\nu}\right)$ can be rewritten as

$$
\begin{aligned}
B_{n, m}\left(\Omega_{k}+\omega_{\nu}, \Omega_{k}\right)= & -\frac{2 \pi \nu}{\sqrt{e H}} \tau^{2} \mathcal{D}_{(2)} T \sum_{\varepsilon_{i}}\left[\sqrt{n+1} \delta_{m, n+1}+\sqrt{n} \delta_{m, n-1}\right] \times \\
& \lambda_{n}\left(\varepsilon_{i}+\omega_{\nu}, \Omega_{k}-\varepsilon_{i}\right) \lambda_{m}\left(\varepsilon_{i}, \Omega_{k}-\varepsilon_{i}\right)
\end{aligned}
$$

with

$$
\lambda_{m}\left(\varepsilon_{1}, \varepsilon_{2}\right)=\frac{1}{\tau} \frac{\Theta\left(-\varepsilon_{1} \varepsilon_{2}\right)}{\left|\varepsilon_{1}-\varepsilon_{2}\right|+\Omega_{L}(m+1 / 2)} .
$$

The critical field $H_{c 2}(T)$ is determined by the equation $L_{0}^{-1}\left(q_{z}=0, \Omega_{k}=\right.$ $0)=0$. This is why in the vicinity of $H_{c 2}(T)$ the singular contribution to (220) originates only from the terms with $L_{0}\left(0, \Omega_{k}\right)$. The frequency dependencies of the functions $B_{n, m}\left(\Omega_{k}+\omega_{\nu}, \Omega_{k}\right)$ and $L_{1}\left(\Omega_{k}\right)$ are weak although we cannot omit them to get nonvanishing answer. It is enough to restrict ourselves to the linear approximation in their frequency dependencies. If the temperature $T \ll$ $T_{c 0}$ the sum over frequencies in (221) can be approximated by an integral. Transforming the boson frequency $\Omega_{k}$ summation to a contour integration as was done above and making the analytic continuation in the external frequency $\omega_{\nu}$ one can get an explicit expression for the d.c. paraconductivity. In the same spirit the contributions of all other diagrams from the Fig. 9 which contribute to fluctuation conductivity in the case under discussion are 
calculated side by side with the AL one. The final answer can be presented in the form:

$$
\begin{aligned}
\delta \sigma_{t o t}= & \frac{2 e^{2}}{3 \pi^{2}}\left\{-\ln \frac{\pi T_{c 0}}{2 \gamma T}+\frac{3 \gamma_{E} T}{T_{c 0}}\left(\frac{H_{c 2}(T)}{H-H_{c 2}(T)}\right)+\right. \\
& +\psi\left[\frac{T_{c 0}}{2 \gamma_{E} T}\left(\frac{H-H_{c 2}(T)}{H_{c 2}(T)}\right)\right]+ \\
& \left.+4\left(\frac{T_{c 0}}{2 \gamma_{E} T} \frac{H_{c 2}(T)}{H-H_{c 2}(T)} \psi^{\prime}\left[\frac{T_{c 0}}{2 \gamma_{E} T}\left(\frac{H-H_{c 2}(T)}{H_{c 2}(T)}\right)\right]-1\right)\right\},
\end{aligned}
$$

where $\gamma_{E}$ is the Euler constant. Let us consider some limiting cases. If the temperature is relatively high $T / T_{c 0} \gg\left(H-H_{c 2}(T)\right) / H_{c 2}(T)$, we obtain the following formula for the fluctuation conductivity:

$$
\delta \sigma=\frac{2 \gamma_{E} e^{2}}{\pi^{2}} \frac{T}{T_{c 0}}\left(\frac{H_{c 2}(T)}{H-H_{c 2}(T)}\right) .
$$

If $H<H_{c 2}(0)$, we can introduce $T_{c}(H)$ and rewrite Eq.(224) in the usual way

$$
\delta \sigma=\frac{3 e^{2}}{2 \gamma_{E} \pi^{2}} \frac{T_{c 0}}{T-T_{c}(H)} .
$$

If $H>H_{c 2}(0)$, in the low-temperature limit $T / T_{c 0} \ll\left(H-H_{c 2}(T)\right) / H_{c 2}(T)$ we have

$$
\delta \sigma=-\frac{2 e^{2}}{3 \pi^{2}} \ln \frac{H_{c 2}(T)}{H-H_{c 2}(T)} .
$$

One can see, that even at zero temperature a logarithmic singularity remains and the corresponding correction is negative. It results from all three fluctuation contributions, although the DOS one exceeds the others by numerical factor. Let us recall that in the case of the c-axis conductivity of a layered superconductor, or in granular superconductors above $T_{c}$, the DOS contribution exceeds the MT and AL ones parametrically 160 .

\subsection{The effect of fluctuations on the Hall conductivity [174]}

Let us start with a discussion of the physical meaning of the Hall resistivity

$\rho_{x y}$. In the case of only one type of carriers it depends on their concentration $n$ and turns out to be independent of the electron diffusion coefficient: $\rho_{x y}=$ $H /(e n)$. The fluctuation processes of the MT and DOS types contribute to the diffusion coefficient, so their expected contribution to the Hall resistivity is zero. For the Hall conductivity in a weak field one can write

$$
\sigma_{x y}=\rho_{x y} \sigma_{x x}^{2}=\rho_{x y} \sigma_{x x}^{(n) 2}+2 \rho_{x y} \sigma_{x x}^{(n)} \delta \sigma_{x x}=\sigma_{x y}^{(n)}\left(1+2 \frac{\delta \sigma_{x x}}{\sigma_{x x}}\right)
$$


so, evidently, the relative fluctuation correction to Hall conductivity is twice as large as the fluctuation correction to the diagonal component. This qualitative speculation is confirmed by the direct calculation of the MT type diagram [169].

The AL process corresponds to an independent charge transfer which cannot be reduced to a renormalization of the diffusion coefficient. It contributes weakly to the Hall effect, and this contribution is related to the Cooper pair particle-hole asymmetry. This effect was investigated in a set of papers: 169, 170, 171, 172, 71, 173, 174. Let us recall that the proper general expression describing the paraconductivity contribution to the Hall conductivity in the general case of arbitrary magnetic fields and frequencies (in the TDGL theory limits) was already carried out above in the phenomenological approach (see Eq. (113)). The microscopic consideration of this value can be done in the spirit of the calculation of $\sigma_{x x}^{A L}$ (see (185)) and after the analytical continuation results in

$$
\begin{aligned}
\sigma_{x y}^{A L}= & \left(\frac{2 h \nu(0)}{\pi}\right)^{2} \sum_{n=0}^{\infty}(n+1) \int_{-\pi / s}^{\pi / s} \frac{d k_{z}}{2 \pi} \int_{-\infty}^{\infty} \operatorname{coth} \frac{z}{2 T} d z \times \\
& \times\left[\operatorname{Im} L_{n}^{R}(z) \frac{\partial}{\partial z} \operatorname{Re} L_{n+1}^{R}(z)-\operatorname{Im} L_{n+1}^{R}(z) \frac{\partial}{\partial z} \operatorname{Re} L_{n}^{R}(z)\right] .
\end{aligned}
$$

where dimensionless magnetic field $h$ was introduced by (77). The phenomenological expression (113) can be obtained from this formula by carrying out the frequency integration in the same way as was done in the calculation of (185) (the essential region of integration is $z \ll T$ ).

One can see from (228) that if $\operatorname{Im} L_{n}^{R}(-z)=-\operatorname{Im} L_{n}^{R}(z)$ and $\operatorname{Re} L_{n}^{R}(-z)=$ $\operatorname{Re} L_{n}^{R}(z)$ the Hall conductivity is equal to zero, or, in terms of the phenomenological parametrization, the reality of $\gamma_{G L}$ results in a zero Hall effect. Physically it is possible to say that this zero is the direct consequence of electron-hole symmetry. However, from the formula (151) one can see that an energy dependence of the density of states or the electron interaction constant $g$ immediately results in the appearance of an imaginary part of $\gamma_{G L}$. In the weak interaction approximation

$$
\operatorname{Im} \gamma_{G L}=\frac{\nu(0)}{2}\left(\frac{\partial \ln T_{c}}{\partial E}\right)_{E=E_{F}} .
$$

Usually this value is small in comparison with $\operatorname{Re} \gamma_{G L}$ by a ratio of the order of $T_{c} / E_{F}$. Taking into account the terms of the order of $\operatorname{Im} \gamma_{G L}$ in (228) and using the explicit form of the fluctuation propagator for layered superconductor (175) one can find

$$
\sigma_{x y}^{A L}=e^{2} T \frac{\operatorname{Im} \gamma_{G L}}{2 \nu(0)} h
$$




$$
\times \int_{-\pi / s}^{\pi / s} \frac{d k_{z}}{2 \pi} \frac{1}{\left.\left[\epsilon+r \sin ^{2}\left(k_{z} s / 2\right)\right)\right]^{2}} F\left(\frac{\left.\epsilon+r \sin ^{2}\left(k_{z} s / 2\right)\right)}{2 h}\right),
$$

where

$$
F(x)=4 x^{2}\left[\psi(x)+x \psi^{\prime}(x)-1-\psi\left(\frac{1}{2}+x\right)\right] . .
$$

For $H \rightarrow 0$ the expression for the fluctuation Hall paraconductivity takes the form

$$
\sigma_{x y}^{A L}=\frac{e^{2} T}{6 s}\left(\frac{\operatorname{Im} \gamma_{G L}}{\nu(0)}\right) h \frac{\epsilon+r / 2}{[\epsilon(\epsilon+r)]^{3 / 2}}=\frac{e^{3} \Phi_{0} T}{6 \pi s}\left(\frac{\operatorname{Im} \gamma_{G L}}{\nu(0)}\right) \frac{\epsilon+r / 2}{[\epsilon(\epsilon+r)]^{3 / 2}} h .
$$

One can see that in the $2 D$ case the temperature dependence of the $\mathrm{AL}$ fluctuation correction to the Hall conductivity

$$
\sigma_{x y}^{A L} \approx \frac{e^{3} \Phi_{0}}{12 \pi s}\left(\frac{T_{c}}{E_{F}}\right) \frac{h}{\epsilon^{2}}
$$

turns out to be more singular than the MT one.

\subsection{Fluctuations in the ultra-clean case 175}

When dealing with the superconductor electrodynamics in the fluctuation regime, it is necessary to remember that in the vicinity of the critical temperature the role of the effective size of a fluctuation Cooper pair is played by the GL coherence length $\xi_{G L}(T)=\xi_{0} / \sqrt{\epsilon}$. So, as was already mentioning above, the case of a pure enough superconductor with electron mean free path $\ell \gg \xi_{0}$ has to be formally subdivided into the clean $\left(\xi_{0} \ll \ell \ll \xi_{G L}(T)\right)$ and ultra-clean $\left(\xi_{G L}(T) \ll \ell\right)$ limits. The nontrivial cancellation of the contributions, previously divergent in $T \tau$ (see, for example, (192)), will be shown in this Section. This results in a reduction of the total fluctuation correction in the ultra-clean case to the AL term only. We will base on 175 restricting our consideration to the case of a $2 \mathrm{D}$ electron system.

In terms of the parameter $T \tau$, used in the theory of disordered alloys, three different domains of the metal purity can be distinguished: $T \tau \ll 1$ (dirty case), $1 \ll T \tau \ll 1 / \sqrt{\epsilon}$ (clean case) and $1 / \sqrt{\epsilon} \ll T \tau$ (ultra-clean case of nonlocal electrodynamics). The latter case was rarely discussed in the literature 150, 176, 177 in spite of the fact that it becomes of primary importance for metals of very modest purity, let us say, with $T \tau \approx 10$. Really, in this case the condition $T \tau \geq 1 / \sqrt{\epsilon}$, which in terms of the reduced temperature is read as $10^{-2} \leq \epsilon \ll 1$, practically covers all the experimentally accessible range of temperatures for the fluctuation conductivity measurements. As regards the usually considered local clean case $(1 \ll T \tau \ll 1 / \sqrt{\epsilon})$ for the chosen value $T \tau \approx 10$, it would not have any range of applicability. Indeed, the equivalent 
condition for the allowed temperature interval is $\epsilon \ll(T \tau)^{-2}$, and it almost contradicts the $2 D$ thermodynamical Ginzburg-Levanyuk criterion for the mean field approximation applicability $\left(G i_{(2)}=\frac{T_{c}}{E_{F}} \ll \epsilon\right)$. Moreover, as we will show below, for transport coefficients the higher order corrections become comparable with the mean field results much before they are important for thermodynamical quantities, namely at $\epsilon \sim \sqrt{G i_{(2)}}$ 178, 179. So in practice one can speak about the dirty and the non-local ultra-clean limits only.

As we saw above the 2D AL contribution turns out to be completely independent of the electron mean free path $\ell$ [5]. The anomalous Maki-Thompson contribution, being induced by the pairing on the Brownian diffusive trajectories [76], naturally depends on $T \tau$, but in an indirect way. It turns out to be $\tau$-independent up to $T \tau \sim 1 / \sqrt{\epsilon}$, (see (201)) and diverges as $T \tau \ln (T \tau)$ for $T \tau \gg 1 / \sqrt{\epsilon} 150,176$. The analogous problem takes place in the case of the DOS contribution: its standard diagrammatic technique calculations lead to a negative correction (192) [95] evidently strongly divergent when $T \tau \rightarrow \infty$. In the derivation of all these results the local form of the fluctuation propagator and Cooperons were used. This is why the direct extension of their validity for $T \tau \gg 1 / \sqrt{\epsilon} \rightarrow \infty$ is incorrect.

One can notice 175 that at the upper limit of the clean case, when $T \tau \sim 1 / \sqrt{\epsilon}$, both the DOS and anomalous MT (201) contributions turn out to be of the same order of magnitude but of opposite signs. So one can suspect that in the case of a correct procedure of impurity averaging in the ultra-clean case the large negative DOS contribution can be cancelled with the positive anomalous MT one. In the case of a $2 D$ electron spectrum the Cooperon can be calculated exactly for the case of an arbitrary electron mean free path:

$$
\lambda\left(\mathbf{q}, \varepsilon_{1}, \varepsilon_{2}\right)=\left(1-\frac{\Theta\left(-\varepsilon_{1} \varepsilon_{2}\right)}{\tau \sqrt{\left(\widetilde{\varepsilon}_{1}-\widetilde{\varepsilon}_{2}\right)^{2}+v_{F}^{2} q^{2}}}\right)^{-1} .
$$

One can see that this expression can be reduced to (168) in the case of $v_{F} q \ll\left|\widetilde{\varepsilon}_{1}-\widetilde{\varepsilon}_{2}\right|$. Let us stress that this result was carried out without any expansion over the Cooper pair center of mass momentum $\mathbf{q}$ and is valid in the $2 D$ case for an arbitrary $\ell q$.

The fluctuation propagator in the $2 D$ case of an arbitrary mean free path can be written as 175

$$
\begin{aligned}
-\left[\nu L\left(\mathbf{q}, \Omega_{k}\right)\right]^{-1}= & \ln \frac{T}{T_{c}}+\sum_{n=0}^{\infty}\left\{\frac{1}{n+1 / 2}\right. \\
& \left.-\frac{1}{\sqrt{\left(n+\frac{1}{2}+\frac{\Omega_{k}}{4 \pi T}+\frac{1}{4 \pi T \tau}\right)^{2}+\frac{v_{F}^{2} \mathbf{q}^{2}}{16 \pi^{2} T^{2}}}-\frac{1}{4 \pi T \tau}}\right\} .
\end{aligned}
$$

Near $T_{c} \ln \frac{T}{T_{c}} \approx \epsilon$ and in the local limit, when only small momenta $\ell q \ll 1$ are involved in the final integrations, the Exp. (234) can be expanded in $v_{F} q / \max \left\{T, \tau^{-1}\right\}$ and reduces to the appropriate local expression. 
Let us demonstrate the specifics of the non-local calculations for the example of the Maki-Thompson contribution. We restrict our consideration to the vicinity of the critical temperature, where the static approximation is valid. Using the non-local expressions for the Cooperon and the propagator one can find after integration over electronic momentum:

$$
\begin{aligned}
Q^{(M T)}\left(\omega_{v}\right)= & -4 \pi \nu v_{F}^{2} e^{2} T^{2} \sum_{\varepsilon_{n}} \int \frac{d^{2} \mathbf{q}}{(2 \pi)^{2}} L(\mathbf{q}, 0) \times \\
& \times\left[\mathcal{M}\left(\tilde{\epsilon}_{n}, \tilde{\epsilon}_{n+\nu}, \mathbf{q}\right)+\mathcal{M}\left(\tilde{\epsilon}_{n+\nu}, \tilde{\epsilon}_{n}, \mathbf{q}\right)\right],
\end{aligned}
$$

where

$$
\mathcal{M}(\alpha, \beta, \mathbf{q})=\frac{R_{q}(2 \alpha) R_{q}(\alpha+\beta)-\Theta(\alpha \beta) R_{q}(2 \alpha) R_{q}(2 \beta)}{(\beta-\alpha)^{2}\left(R_{q}(2 \alpha)-\frac{1}{\tau}\right)\left(R_{q}(2 \beta)-\frac{1}{\tau}\right) R_{q}(\alpha+\beta)}
$$

and $R_{q}(x)=\sqrt{x^{2}+v_{F}^{2} \mathbf{q}^{2}}$. The analogous consideration of the DOS diagrams 2 and 4 which are the leading ones in the clean case [95] results in similar expressions.

One can see that, after analytical continuation over the external frequency $\omega_{\nu} \rightarrow-i \omega$ and the consequent tending $\omega \rightarrow 0$, each of the DOS or MT type diagrams is written in the form of a Laurent series of the type $C_{-2}(T \tau)^{2}+$ $C_{-1}(T \tau)+C_{0}+C_{1}(T \tau)^{-1}+\ldots$ and is divergent at $T \tau \rightarrow \infty$ in accordance with (192). Nevertheless the expansion in a Laurent series of the sum of these nonlocal diagrams leads to the exact cancellation of all divergent contributions. The leading order of the sum of the MT and DOS contributions in the limit of $T \tau \gg 1$ turns out to be proportional to $(T \tau)^{-1}$ only and disappears in the ultra-clean limit. So the correct accounting for non-local scattering processes in the ultra-clean limit results in a total quantum correction negligible in comparison with the AL contribution. Nevertheless, its formal independence on impurities concentration (see (99)) was re-examined for ultra-clean case too in 174 and there it was demonstrated that this statement is valid in a rigorous sense only in the case of direct current and absence of a magnetic field. Let us recall that the normal Drude conductivity in the ultra-clean case takes the form

$$
\sigma_{ \pm}(\omega)=\sigma_{x x} \pm i \sigma_{x y}=\frac{e^{2} n \tau / m}{1-i\left(\omega \mp \omega_{c}\right) \tau}
$$

where $\omega_{c}$ is the cyclotron frequency. When $\tau \rightarrow \infty$ the real part of the conductivity vanishes. The analysis of the AL diagram in the ultra-clean case demonstrates that each of the Green functions blocks $B$ acquires the same denominator. As a result the expression for the fluctuation conductivity contains the same Drude like pole but of second order

$$
\sigma_{ \pm}^{A L}(\omega)=\frac{\sigma_{x x}^{A L(l)} \pm i \sigma_{x y}^{A L(l)}}{\left(1-i\left(\omega \mp \omega_{c}\right) \tau\right)^{2}}
$$


$\left(\sigma_{\alpha \beta}^{A L(l)}\right.$ is the component of the paraconductivity tensor calculated above in the local limit (99-(232)). The origin of this pole can be recognized by means of the following speculation. The electric field does not interact directly with the fluctuation Cooper pairs, but it produces the effect by interaction with the quasiparticles forming these pairs only. The characteristic time of the change of a quasiparticle state is of the order of $\tau$. Consequently the single-particle Drude type conductivity in an a.c. field has a first order pole, while in the AL paraconductivity it is of second order [174]. In spite of this difference one can see that the AL conductivity, like the Drude one, vanishes at $\omega \neq 0, \tau \rightarrow \infty$ because in the absence of impurities the interaction of the electrons does not produce any effective force acting on the superconducting fluctuations, while the d.c. paraconductivity conserves its usual $\tau$-independent form. It is impossible to distinguish the motion of the electron liquid from the condensate motion in current experiments without additional scattering.

The non-local form of the Cooperon and fluctuation propagator have to be taken into account not only for the ultra-clean case but in every problem where relatively large bosonic momenta are involved: the consideration of dynamical and short wavelength fluctuations beyond the vicinity of critical temperature, the effect of relatively strong magnetic fields on fluctuations etc. Recently such an approach was developed in a number of studies 176, $175,180,181$.

\subsection{The effect of fluctuations on the one-electron density of states and on tunneling measurements}

Density of states [94]. The appearance of non-equilibrium Cooper pairing above $T_{c}$ leads to a redistribution of the one-electron states around the Fermi level. A semi-phenomenological study of the fluctuation effects on the density of states (DOS) of a dirty superconducting material was first carried out while analyzing the tunneling experiments of granular $A l$ in the fluctuation regime just above $T_{c} 182$. The second metallic electrode was in the superconducting regime and its well developed gap gave a bias voltage around which a structure, associated with the superconducting fluctuations of $A l$, appeared. The measured DOS energy dependence has a dip at the Fermi level 27, reaches its normal value at some energy $E_{0}(T)$, show a maximum at an energy value equal to several times $E_{0}$, finally decreases towards its normal value at higher energies. The characteristic energy $E_{0}$ was found to be of the order of the inverse of the GL relaxation time $\tau_{G L}$ introduced above.

The presence of a depression at $E=0$ and of a peak at $E \sim\left(1 / \tau_{G L}\right)$ in the DOS above $T_{c}$ are precursor effects of the appearance of the superconducting gap in the quasiparticle spectrum at temperatures below $T_{c}$. The microscopic calculation of the fluctuation contribution to the one-electron DOS can be carried out within the diagrammatic technique 993,94].

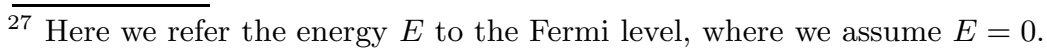




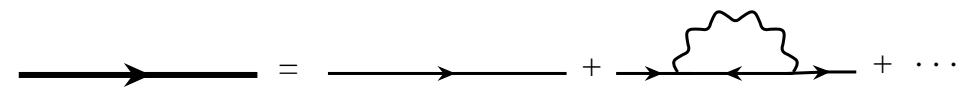

Fig. 15. The one-electron Green function with the first order fluctuation correction.

Let us start from the discussion of a clean superconductor. As is well known the one-electron DOS is determined by the imaginary part of the retarded Green function integrated over momentum. This definition permits us to express the appropriate fluctuation correction in terms of the fluctuation propagator:

$$
\delta \nu^{(c)}(E, \epsilon)=-\frac{1}{\pi} \operatorname{Im} \int \frac{d^{D} \mathbf{p}}{(2 \pi)^{D}} \delta G^{R}(\mathbf{p}, E)=-\frac{1}{\pi} \operatorname{Im} R^{R}(E)
$$

where $R^{R}(E)$ is the retarded analytical continuation of the expression corresponding to the diagram of Fig. 15:

$$
R\left(\varepsilon_{n}\right)=T \sum_{\Omega_{k}} \int \frac{d^{D} \mathbf{q}}{(2 \pi)^{D}} L\left(\mathbf{q}, \Omega_{k}\right) \int \frac{d^{D} \mathbf{p}}{(2 \pi)^{D}} G^{2}\left(\mathbf{p}, \varepsilon_{n}\right) G\left(\mathbf{q}-\mathbf{p}, \Omega_{k}-\varepsilon_{n}\right) .
$$

The result of the integration of the last expression depends strongly of the electron spectrum dimensionality: for the two important cases of isotropic $3 D$ and $2 D$ electron spectra one finds 94

$$
\begin{aligned}
\frac{\delta \nu_{(3)}^{(c)}(E, \epsilon)}{\nu_{(3)}(0)}= & -\frac{(4 \pi)^{3 / 2}}{7 \zeta(3)} \sqrt{G i_{(3, c)}} \operatorname{Re} \frac{\sqrt{T_{c}}}{\sqrt{\tau_{G L}^{-1}-2 i E+\varkappa_{3}^{2} T_{c}}} \times \\
& \left\{\frac{1}{\tau_{G L}^{-1}-i E+\tau_{G L}^{-1 / 2}\left[\tau_{G L}^{-1}-2 i E+\varkappa_{3}^{2} T_{c}\right]^{1 / 2}}\right\}, \\
\frac{\delta \nu_{(2)}^{(c)}(E, \epsilon)}{\nu_{(2)}(0)}=- & -\frac{(4 \pi)^{2}}{7 \zeta(3)} G i_{(2, c)} \frac{T_{c}^{2}}{\left[E^{2}+\varkappa_{2}^{2} T_{c} \tau_{G L}^{-1}\right]} \times \\
& \left\{\frac{E}{\sqrt{E^{2}+\varkappa_{2}^{2} T_{c} \tau_{G L}^{-1}}} \ln \frac{E+\sqrt{E^{2}+\varkappa_{2}^{2} T_{c} \tau_{G L}^{-1}}}{\varkappa \sqrt{T_{c} \tau_{G L}}}\right\},
\end{aligned}
$$

where $\varkappa_{D}=\pi \sqrt{\pi D / 7 \zeta(3)}$.

In a dirty superconductor the calculations may be carried out in a similar way with the only difference that the impurity renormalization of the Cooper 


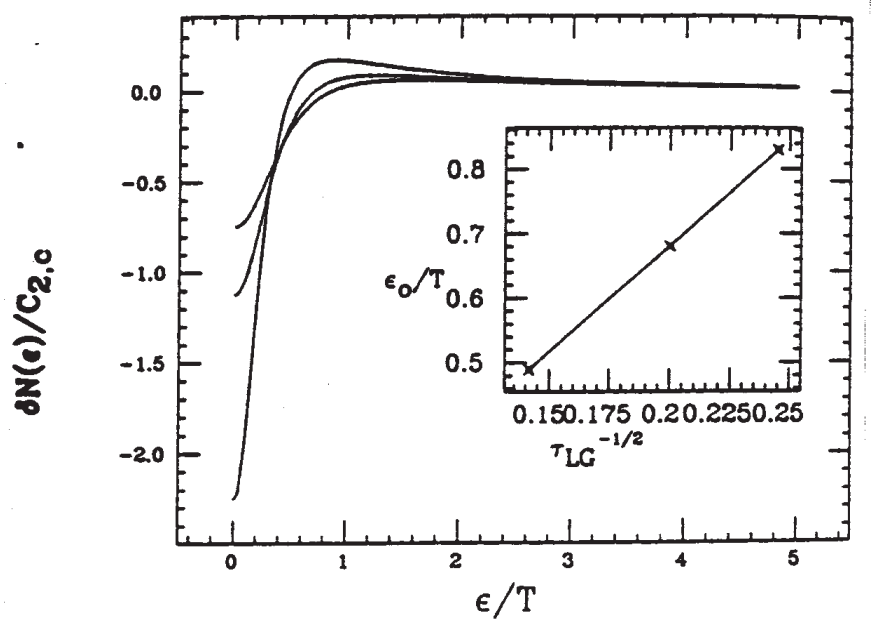

Fig. 16. The theoretical curve of the energy dependence for the normalised correction to the single-particle density of states vs energy for a clean two-dimensional superconductor above $T_{c}$.

vertices has to be taken into account 93. The value of the fluctuation dip at the Fermi level can be written in the form:

$$
\frac{\delta \nu^{(d)}(0)}{\nu(0)} \sim-\left\{\begin{array}{ll}
\sqrt{G i_{(3, d)}} \epsilon^{-3 / 2}, & D=3 \\
G i_{(2, d)} \epsilon^{-2}, & D=2
\end{array} .\right.
$$

At large energies $E \gg \tau_{G L}^{-1}$ the DOS recovers its normal value, according to the same laws (242) but with the substitution $\epsilon \rightarrow E / T_{c}$. It is interesting that the critical exponents of the fluctuation correction of the DOS change when moving from a dirty to a clean superconductor [94]: the analysis of (240)-(241) gives

$$
\frac{\delta \nu^{(c)}(0)}{\nu(0)} \sim-\left\{\begin{array}{ll}
\sqrt{G i_{(3, c)}} \epsilon^{-1 / 2}, & D=3 \\
G i_{(2, c)} \epsilon^{-1}, & D=2
\end{array} .\right.
$$

Another important respect in which the character of the DOS renormalization differs strongly for the clean and dirty cases is the energy scale at which this renormalization occurs. In the dirty case this energy turns out to be 93] $E_{0}^{(d)} \sim T-T_{c} \sim \tau_{G L}^{-1}$, while in the clean case $E_{0}^{(c)} \sim \sqrt{T_{c}\left(T-T_{c}\right)}$ 94]. To understand this important difference one has to study the character of the electron motion in both cases [94]. The relevant energy scale in the dirty case is the inverse of the time necessary for the electron to diffuse over a distance equal to the coherence length $\xi(T)$. This energy scale coincides with the inverse relaxation time: $t_{\xi}^{-1}=\mathcal{D} \xi^{-2}(T) \sim \tau_{G L}^{-1} \sim T-T_{c}$. In the clean case, the ballistic motion of the electrons gives rise to a different characteristic energy scale $t_{\xi}^{-1} \sim v_{F} \xi^{-1}(T) \sim\left(T_{c} \tau_{G L}^{-1}\right)^{1 / 2} \sim \sqrt{T_{c}\left(T-T_{c}\right)}$. 
One can check that the integration of (240)-(241) over all positive energies gives zero:

$$
\int_{0}^{\infty} \delta \nu(E) d E=0
$$

This "sum rule" is a consequence of a conservation law: the number of quasiparticles is determined by the number of cells in the crystal and cannot be changed by the interaction. So the only effect which can be produced by the inter-electron interaction is a redistribution of the energy levels near the Fermi energy. The sum rule (244) plays an important role in the understanding of the manifestation of the fluctuation DOS renormalization in the observable phenomena. As we will see in the next Section the singularity in the tunneling current (at zero voltage), due to the density of states renormalization, turns out to be much weaker than that in the DOS itself (ln $\epsilon$ instead of $\epsilon^{-1}$ or $\epsilon^{-2}$, see (242)-(243)). A similar smearing of the DOS singularity occurs in the opening of the pseudo-gap in the c-axis optical conductivity, in the NMR relaxation rate etc. These features are due to the fact that we must always form the convolution of the DOS with some slowly varying function: for example, a difference of Fermi functions in the case of the tunnel current. The sum rule then leads to an almost perfect cancellation of the main singularity at low energies. The main non-zero contribution then comes from the high energy region where the DOS correction has its 'tail'. Another important consequence of the conservation law (244) is the considerable increase of the characteristic energy scale of the fluctuation pseudo-gap opening with respect to $E_{0}$ : this is $e V_{0}=\pi T$ for tunneling and $\omega \sim \tau^{-1}$ for the c-axis optical conductivity.

The effect of fluctuations on the tunnel current [179]. It is quite evident that the renormalization of the density of states near the Fermi level, even of only one of the electrodes, will lead to the appearance of anomalies in the voltage-current characteristics of a tunnel junction. The quasiparticle current flowing through it may be written as a convolution of the densities of states with the difference of the electron Fermi distributions in each electrode (L and $\mathrm{R}$ ):

$$
\begin{aligned}
I_{q p}= & \frac{1}{e R_{n} \nu_{L}(0) \nu_{R}(0)} \times \\
& \int_{-\infty}^{\infty}\left(\tanh \frac{E+e V}{2 T}-\tanh \frac{E}{2 T}\right) \nu_{L}(E) \nu_{R}(E+e V) d E,
\end{aligned}
$$

where $R_{n}$ is the Ohmic resistance per unit area and $\nu_{L}(0), \nu_{R}(0)$ are the densities of states at the Fermi levels in each of electrodes in the absence of interaction. One can see that for low temperatures and voltages the expression in parenthesis is a sharp function of energy near the Fermi level. Nevertheless, 
depending on the properties of the DOS functions, the convolution (245) may exhibit different properties. If the energy scale of the DOS correction is much larger than $T$, the expression in parenthesis in (245) acts as a delta-function and the zero-bias anomaly in the tunnel conductivity strictly reproduces the anomaly of the density of states around the Fermi level:

$$
\frac{\delta G(V)}{G_{n}(0)}=\frac{\delta \nu(e V)}{\nu(0)},
$$

where $G(V)$ is the differential tunnel conductance and $G_{n}(0)$ is the background value of the Ohmic conductance supposed to be bias independent, $\delta G(V)=G(V)-G_{n}(0)$. This situation, for instance, occurs in a junction with one amorphous electrode 183, where the dynamically screened Coulomb interaction is strongly retarded, which leads to a considerable suppression of the density of states in the vicinity of the Fermi level, within $\tau^{-1} \gg T$.

It is worth stressing that the proportionality between the tunneling current and the electron DOS of the electrodes is widely accepted as an axiom, but generally speaking this is not always so. As one can see from the previous subsection, the opposite situation occurs in the case of the DOS renormalization due to the electron-electron interaction in the Cooper channel: in this case the DOS correction varies strongly already in the scale of $E_{0} \sim E_{\mathrm{ker}} \ll T$ and the convolution in (245) with the DOS (241) has to be carried out without the simplifying approximations assumed to obtain (246). We will show that the fluctuation induced pseudo-gap like structure in the tunnel conductance differs drastically from the anomaly of the density of states (241), both in its temperature singularity near $T_{c}$ and in the energy range of its manifestation.

Let us first discuss the effect of the fluctuation suppression of the density of states on the properties of a tunnel junction between a normal metal and a superconductor above $T_{c}$. The effect under discussion turns out to be most pronounced in the case of thin superconducting films $(d \ll \xi(T))$ and layered superconductors like HTS cuprates. In order to derive the explicit expression for the fluctuation contribution to the differential conductance of a tunnel junction with one thin film electrode close to its $T_{c}$ we differentiate (245) with respect to voltage, and substitute the DOS correction given by (241). This results in (see [179):

$$
\begin{aligned}
\frac{\delta G_{f l}(V, \epsilon)}{G_{n}(0)} & =\frac{1}{2 T} \int_{-\infty}^{\infty} \frac{d E}{\cosh ^{2}\left(\frac{E+e V}{2 T}\right)} \delta \nu^{(2)}(E, \epsilon) \sim \\
& \sim G i_{(2)} \ln \left(\frac{2}{\sqrt{\epsilon}+\sqrt{\epsilon+r}}\right) \operatorname{Re} \psi^{\prime \prime}\left(\frac{1}{2}-\frac{i e V}{2 \pi T}\right) .
\end{aligned}
$$

It is important to emphasize several nontrivial features of the result obtained. First, the sharp decrease $\left(\epsilon^{-2(1)}\right)$ of the density of electron states in the immediate vicinity of the Fermi level generated by fluctuations surprisingly results in a much more moderate growth of the tunnel resistance at zero 


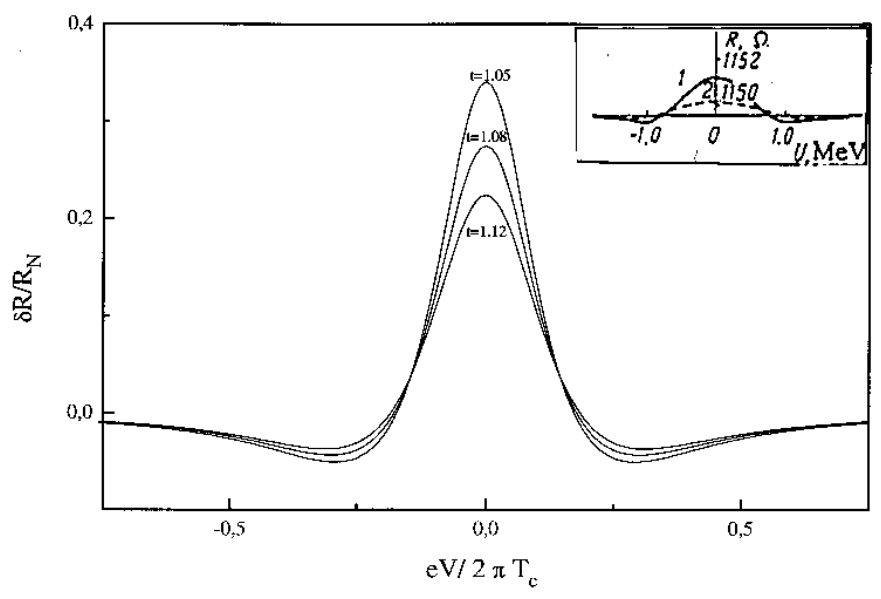

Fig. 17. The theoretical prediction for the fluctuation-induced zero-bias anomaly in tunnel-junction resistance as a function of voltage for reduced temperatures $\epsilon=0.05$ (top curve), $\epsilon=0.08$ (middle curve) and $\epsilon=0.12$ (bottom curve). The insert shows the experimentally observed differential resistance as a function of voltage in an Al-I-Sn junction just above the transition temperature

voltage (ln $1 / \epsilon)$. Second, in spite of the manifestation of the DOS renormalization at the characteristic scales $E_{0}^{(d)} \sim T-T_{c}$ or $E_{0}^{(c l)} \sim \sqrt{T_{c}\left(T-T_{c}\right)}$, the energy scale of the anomaly developed in the $I-V$ characteristic is much larger: $\mathrm{eV}=\pi T \gg E_{0}$ (see Fig. 17).

In the inset of Fig. 17 the result of measurements of the differential resistance of the tunnel junction $A l-I-S n$ at temperatures slightly above the critical temperature of $S n$ electrode is presented. This experiment was done 184 with the purpose of checking the theory proposed 179]. The nonlinear differential resistance was precisely measured at low voltages which permitted the observation of the fine structure of the zero-bias anomaly. The reader can compare the shape of the measured fluctuation part of the differential resistance (the inset in Fig. 17) with the theoretical prediction. It is worth mentioning that the experimentally measured positions of the minima are $e V \approx \pm 3 T_{c}$, while the theoretical prediction following from (247) is $e V= \pm \pi T_{c}$. Recently similar results on an aluminium film with two regions of different superconducting transition temperatures were reported 185. The observations of the pseudogap anomalies in tunneling experiments at temperatures above $T_{c}$ obtained by a variety of experimental techniques were reported in 186, 187, 188, 189, 190]..

We will now consider the case of a symmetric junction between two superconducting electrodes at temperatures above $T_{c}$. In this case, evidently, the correction (247) has to be multiplied by a factor of "two" because of the possibility of fluctuation pairing in both electrodes. Furthermore, in view 
of the extraordinarily weak $(\sim \ln 1 / \epsilon)$ temperature dependence of the first order correction, different types of high order corrections may manifest themselves on the energy scale $\mathrm{eV} \sim T-T_{c}$ or $\sqrt{T_{c}\left(T-T_{c}\right)}$. Among them are the familiar AL and MT corrections which take place in the first order of $G i$ but in the second order of the barrier transparency. Another type of higher order correction appears in the first order of barrier transparency but in the second of fluctuation strength $\left(\sim G i^{2}\right)$ [179. Such corrections are generated by the interaction of fluctuations through the barrier and they can be evaluated directly from (245) applied to a symmetric junction. The second order correction in $G i$ can be written as 179:

$$
\delta G_{f l}^{(2)}(0, \epsilon) \sim \int_{-\infty}^{\infty} \frac{d E}{\cosh ^{2}\left(\frac{E}{2 T}\right)}\left[\delta \nu^{(2)}(E, \epsilon)\right]^{2} \sim \frac{G i_{(2)}^{2}}{\epsilon^{3}}
$$

This nonlinear fluctuation correction turns out to be small by $G i^{2}$ but its strong singularity in temperature and opposite sign with respect to $\delta G_{f l}^{(1)}$ make it interesting. Apparently it leads to the appearance of a sharp maximum at zero voltage in $G(V)$ with a characteristic width $e V \sim T-T_{c}$ in the immediate vicinity of $T_{c}$ (one can call this peak as the hyperfine structure). This result was confirmed in 191] but to our knowledge such corrections were never observed in tunneling experiments.

One can see that $\delta G_{f l}^{(1)}$ and $\delta G_{f l}^{(2)}$ become of the same order at $\epsilon_{c r}^{*} \sim \sqrt[3]{G i}$, i.e. the critical region where nonlinear fluctuations effects become important in the problem under consideration starts much before the thermodynamical criterion $\epsilon_{c r} \sim G i$. In the next Section we will discuss this early manifestation of nonlinear fluctuation effects in transport phenomena.

\subsection{Nonlinear fluctuation effects 14}

As we have already seen in the temperature region $G i \ll \epsilon \ll 1$ the thermodynamic fluctuations of the order parameter $\Psi$ can be considered to be Gaussian. Nevertheless the example of the previous Section demonstrates that in transport phenomena nonlinear effects, related with the interaction of fluctuations (higher order corrections) can manifest themselves much earlier. It has been found in paper [178], that nonlinear fluctuation phenomena restrict the Gaussian region in the fluctuation conductivity of a superconducting film to a new temperature scale: $\sqrt{G i_{(2 d)}} \ll \epsilon \ll 1$ (see also [110,111, 179, 115, 192). In this Section we obtain expressions for the conductivity in the temperature region $G i_{(2 d)}<\epsilon<\sqrt{G i_{(2 d)}}$, where both the perturbation theory works well and the nonlinear fluctuation effects are important.

Let us start from the correlator (173) which can be expressed by means of the $G i_{(2 d)}$ number:

$$
\left\langle\Psi_{\mathbf{k}}^{*} \Psi_{\mathbf{k}}\right\rangle=\frac{T}{\nu} \frac{1}{\epsilon+\frac{\pi \mathcal{D}}{8 T} \mathbf{k}^{2}}=\frac{32 \pi^{3}}{7 \zeta(3)} G i_{(2 d)} \frac{T^{2}}{k^{2}+\frac{8 T \epsilon}{\pi \mathcal{D}}} .
$$


The long-wave-length fluctuations with $k^{2}<k_{\min }^{2}=8 T \epsilon / \pi \mathcal{D}$ can be considered as a local condensate. They lead to the formation of the pseudogap

$$
\Delta_{p g}=\left[\int_{k^{2} \lesssim k_{\min }^{2}} \frac{d^{2} k}{(2 \pi)^{2}}\left\langle\Psi_{\mathbf{k}}^{*} \Psi_{\mathbf{k}}\right\rangle\right]^{1 / 2} \simeq T \sqrt{G i_{(2 d)}} .
$$

in the single-particle spectrum of excitations.

Not very close to the transition $\left(\epsilon>\sqrt{G i_{(2 d)}}\right)$ only excitations with energies $E>\Delta_{p g}$ are important. The pseudogap does not play any role for them. Thus, in this region of temperatures it is sufficient to consider fluctuations in the linear approximation only (see [5,6,7]). However, in the temperature region $\epsilon<\sqrt{G i_{(2 d)}}$ the nonlinear fluctuation contribution of the excitations with energies $E<\Delta_{p g}$ becomes essential.

To take into account the spatial dependence of the order parameter we will use the results obtained in 193]. It was shown there that the spatial variations of $\Delta_{p g}$ act on single-particle excitations in the same way as magnetic impurities do (the analogy between the effect of fluctuations and magnetic impurities was observed in many papers, see for example, 194). In this case, the total pairbreaking rate $\Gamma$ can be written as a sum of the pairbreaking rate due to the magnetic impurities and the fluctuation term. Thus, the selfconsistent equation for $\Gamma$ can be written in the following form [193]:

$$
\Gamma=\int \frac{d^{2} k}{(2 \pi)^{2}} \frac{\left\langle\Psi_{k}^{*} \Psi_{k}\right\rangle}{E+\frac{1}{2} \mathcal{D} k^{2}+\Gamma}+\frac{1}{\tau_{s}} .
$$

In the region $E \lesssim \Gamma, \Gamma \gg T \epsilon$ we obtain from Eqs.(251), (249):

$$
\Gamma \sim T\left(G i_{(2 d)}\right)^{1 / 2} \simeq \Delta_{p g},
$$

which coincides with the results obtained in 110,101.

Let us note, that the pair-breaking rate $\Gamma$ was found to be of the order of the pseudogap $\Delta_{p g}$. Thus, a wide maximum appears in the density of states at $E \sim \Delta_{p g}$. As we already saw (201), in purely $2 D$ case the Maki-Thompson correction to the conductivity saturates for $T \epsilon<\Gamma$ (where $\Gamma=8 T \gamma_{\varphi} / \pi$ ) and takes the form [14:

$$
\frac{\delta \sigma^{M T}}{\sigma_{n}} \sim \frac{T}{\Gamma} G i_{(2 d)} \ln \frac{\pi \Gamma}{8 T \epsilon} .
$$

As it can be seen from Eqs. (252) such a saturation takes place when $\epsilon<$ $\sqrt{G i_{(2 d)}}$. Similar results have been obtained in [110,111, 115, with slightly different numerical coefficients ${ }^{28}$. However, its exact value is not very important since in the region $T \epsilon<\Gamma$ the Maki-Thompson correction is less singular

${ }^{28}$ Note, that the numerical coefficient in Eq. 253 depends on the definition of $G i_{(2 d)}$ and how the summation of higher order diagrams is made. 
than the Aslamazov-Larkin one and can be neglected. The latter does not saturate when $T$ tends to $T_{c}$ but becomes more and more singular.

In the presence of the pseudogap if there is no equilibrium, the fluctuating Cooper pair lifetime increases with respect to the GL one: $\tau_{f l}=a \tau_{G L}$ $(a>1)$. Recall, that analogous changes in the coefficient $a$ in the TDGL equations appear below the transition temperature (see e.g. 66, 72, 195, 196, 197]). The growth of the coefficient $a$ and, consequently, the increase of the fluctuation lifetime is because the quasiparticles require more time to attain thermal equilibrium (the corresponding time we denote as $\tau_{e}$ ). A rough estimate gives $a \sim \Delta_{p g} \tau_{e}$. In the case of weak energy relaxation, $\tau_{e}$ has to be determined from the diffusion equation taking account of the pseudogap (see [196, 197, 198]). Note, that in this complicated case the coefficient $a$ becomes a non-local operator. Rough estimates give the following value for the thermal equilibrium transition time $\tau_{e} \sim\left(\mathcal{D} k_{\min }^{2}\right)^{-1} \sim(T \epsilon)^{-1}$. Taking into account Eq. 250) we obtain from (11) for the paraconductivity contribution in the discussed limit of the weak energy relaxation [14] :

$$
\frac{\delta \sigma}{\sigma_{n}} \sim \frac{G i_{(2 d)}^{3 / 2}}{\epsilon^{2}} .
$$

Let us discuss now the role of the energy relaxation processes, characterized by a quasiparticle lifetime $\tau_{\varepsilon}$. Nonelastic electron scattering off phonons and other possible collective excitations can decrease $\tau_{\varepsilon}$ significantly. These processes together with additional pairbreaking processes (due to magnetic impurities or a magnetic field) lead to a decrease of the nonlinear effects. In view of these processes, one can write the following interpolation formula for the non-linear fluctuation conductivity [14]:

$$
\frac{\delta \sigma}{\sigma_{n}} \sim \frac{G i_{(2 d)}^{3 / 2}}{\epsilon\left(\epsilon+\frac{1}{T \tau_{\varepsilon}}\right)\left(1+\frac{\Gamma}{T \sqrt{G i_{(2 d)}}}\right)} .
$$

Note that Eqs. 254 255) are valid only if the parameters $\Gamma$ and $\tau_{\varepsilon}$ are such that the correction to conductivity $\delta \sigma$ is larger than the usual AslamazovLarkin correction Eq.999). If $\Gamma>T, T \tau_{\varepsilon}<\sqrt{G i_{(2 d)}}$ or if $T^{2} \tau_{\varepsilon} / \Gamma<G i_{(2 d)}$, than nonlinear effects are negligible and the usual result (99) is valid for all $\epsilon>G i_{(2 d)}$.

We see that the paraconductivity can exceed the value of the normal conductivity $\sigma_{n}$ in the region $G i_{(2 d)}<\epsilon<G i_{(2 d)}^{3 / 4}$. Let us recall, that in this region corrections to all the thermodynamic coefficients are still small and the linear theory is well applicable.

\subsection{The effect of fluctuation on the optical conductivity [199]}

The optical conductivity of a layered superconductor can be expressed by the same analytically continued electromagnetic response operator $Q_{\alpha \beta}^{(R)}(\omega)$ 
(see Exp.(180) but in contrast to the d.c. conductivity case, calculated without the assumption $\omega \rightarrow 0$. Let us recall that the paraconductivity tensor in an a.c. field was already studied in Section 4 in the framework of the TDGL equation [31] and the most interesting asymptotics for our discussion (125)-(126), valid for $\omega \ll T$ in the $2 D$ regime, were calculated there. The microscopic calculation of the AL diagram [98] shows that in the vicinity of $T_{c}$ and for $\omega \ll T$ the leading singular contribution to the response operator $Q_{\alpha \beta}^{A L(R)}$ arises from the fluctuation propagators rather than from the $B_{\alpha}$ blocks, which confirms the TDGL results. Nevertheless the DOS and MT corrections can be calculated only by the microscopic method, as was done in 98,199 ..

Let us note that the external frequency $\omega_{\nu}$ enters in the expression for the DOS contribution to $Q_{\alpha \beta}(\omega)$ only by means of the Green's function $G\left(\mathbf{p}, \omega_{n+\nu}\right)$ and it is not involved in $q$ integration. So, near $T_{c}$, even in the case of an arbitrary external frequency, we can restrict consideration to the static limit, taking into account only the propagator frequency $\Omega_{k}=0$, and to get 199:

$$
\operatorname{Re} \sigma_{\alpha \beta}^{D O S}(\omega)=-\frac{e^{2}}{2 \pi s} \hat{\kappa}(\omega, T, \tau) A_{\alpha \beta} \ln \left[\frac{2}{\sqrt{\epsilon+r}+\sqrt{\epsilon}}\right],
$$

where the anisotropy tensor $A_{\alpha \beta}$ was introduced in 189 . Let us stress that, in contrast to the AL frequency dependent contribution, this result has been found with only the assumption $\epsilon \ll 1$, so it is valid for any frequency, and impurity concentration. The function $\hat{\kappa}(\omega, T, \tau)$ was calculated in 199. exactly but we present here only its asymptotics for the clean and dirty cases:

$$
\begin{aligned}
& \hat{\kappa}_{d}\left(\omega, T \ll \tau^{-1}\right)=\frac{8}{\pi} \begin{cases}\frac{7 \zeta(3)}{2 \pi^{2}}, & \omega \ll T \ll \tau^{-1} \\
\left(\frac{T}{\omega}\right)^{2}, & T \ll \omega \ll \tau^{-1}, \\
-\frac{\pi T^{2}}{\omega^{3} \tau}, & T \ll \tau^{-1} \ll \omega\end{cases} \\
& \hat{\kappa}_{c l}\left(\omega, T \gg \tau^{-1}\right)=\frac{\pi^{3}}{28 \zeta(3)}\left\{\begin{array}{cl}
(T \tau)^{2}, & \omega \ll \tau^{-1} \ll T \\
\left(\frac{T}{\omega}\right)^{2}, & \tau^{-1} \ll \omega \ll T \\
-4\left(\frac{T}{\omega}\right)^{3}, \tau^{-1} \ll T \ll \omega
\end{array} .\right.
\end{aligned}
$$

The general expression for the MT contribution is too cumbersome, so we restrict ourselves here to the important $2 D$ overdamped regime $\left(r \ll \epsilon \leq \gamma_{\varphi}\right)$ :

$$
\begin{aligned}
& \sigma_{z z}^{M T(a n)(2 D)}(\omega)=\frac{e^{2} s}{2^{7} \eta_{(2)}} \frac{r^{2}}{\gamma_{\varphi} \epsilon}\left\{\begin{array}{c}
1, \quad \tilde{\omega} \ll \tau_{\varphi}^{-1} \\
\left(\frac{8 T_{c} \gamma_{\varphi}}{\pi \omega}\right)^{2}, \tilde{\omega} \gg \tau_{\varphi}^{-1}
\end{array}\right. \\
& \sigma_{x x}^{M T(a n)(2 D)}(\omega)=\frac{e^{2}}{8 s}\left\{\begin{array}{cl}
\frac{1}{\gamma_{\varphi}} \ln \frac{\gamma_{\varphi}}{\epsilon}, \omega \ll \tau_{\varphi}^{-1} \\
\left(\frac{8 T_{c} \gamma_{\varphi}}{\pi \omega}\right)^{2}, \quad \omega \gg \tau_{\varphi}^{-1} .
\end{array}\right.
\end{aligned}
$$


Let us discuss the results obtained. Because of the large number of parameters entering the expressions we restrict our consideration to the most interesting $c$-axis component of the fluctuation conductivity tensor in the $2 D$ region (above the Lawrence-Doniach crossover temperature).

The AL contribution describes the fluctuation condensate response to the applied electromagnetic field. The current associated with it can be treated as the precursor phenomenon of the screening currents in the superconducting phase. As was demonstrated above the characteristic "binding energy " of fluctuation Cooper pair is of the order of $T-T_{c}$, so it is not surprising that the AL contribution decreases when the electromagnetic field frequency exceeds this value. Indeed $\omega^{A L} \sim T-T_{c}$ is the only relevant scale for $\sigma^{A L}$ : its frequency dependence does not contain $T, \tau_{\varphi}$ and $\tau$. The independence from the latter is due to the fact that elastic impurities do not present obstacles for the motion of Cooper pairs. The interaction of the electromagnetic wave with the fluctuation Cooper pairs resembles, in some way, the anomalous skin-effect where the reflection is determined by the interaction with the free electron system.

The anomalous MT contribution also is due to fluctuation Cooper pairs, but this time they are formed by electrons moving along self-intersecting trajectories. Being the contribution related with the Cooper pair electric charge transfer it does not depend on the elastic scattering time but it turns out to be extremely sensitive to the phase-breaking mechanisms. So two characteristic scales turn out to be relevant in its frequency dependence: $T-T_{c}$ and $\tau_{\varphi}^{-1}$. In the case of HTS, where $\tau_{\varphi}^{-1}$ has been estimated as at least $0.1 T_{c}$, for temperatures up to $5 \div 10 \mathrm{~K}$ above $T_{c}$ the MT contribution is overdamped, it is determined by the value of $\tau_{\varphi}$ and is almost temperature independent.

The DOS contribution to $\operatorname{Re} \sigma(\omega)$ is quite different from those above. In the wide range of frequencies $\omega \ll \tau^{-1}$ the lack of electron states at the Fermi level leads to the opposite sign effect in comparison with the AL and MT contributions: $\operatorname{Re} \sigma^{D O S}(\omega)$ turns out to be negative and this means an increase of the surface impedance, or, in other words, decrease of the reflectance. Nevertheless, the applied electromagnetic field affects the electron distribution and at very high frequencies $\omega \sim \tau^{-1}$ the DOS contribution changes its sign. It is interesting that the DOS contribution, as a one-electron effect, depends on the impurity scattering in a similar manner to the normal Drude conductivity. The decrease of $\operatorname{Re} \sigma^{D O S}(\omega)$ starts at frequencies $\omega \sim$ $\min \left\{T, \tau^{-1}\right\}$ which for HTS are much higher than $T-T_{c}$ and $\tau_{\varphi}^{-1}$.

The $\omega$-dependence of $\operatorname{Re} \sigma_{z z}^{t o t}$ with the most natural choice of parameters $\left(T_{c} r \ll T_{c} \epsilon \leq \tau_{\varphi}^{-1} \ll \min \left\{T, \tau^{-1}\right\}\right)$ is presented in Fig. 18 .

Let us discuss it referring to a strongly anisotropic layered superconductor. The positive AL and MT contributions to $\sigma_{z z}^{\text {tot }}$, being suppressed by the square of the interlayer transparency, are small in magnitude and they vary in the low frequency region $\omega \sim \min \left\{T-T_{c}, \tau_{\varphi}^{-1}\right\}$. The DOS contribution is proportional to the first order of transparency and remains in this region almost 


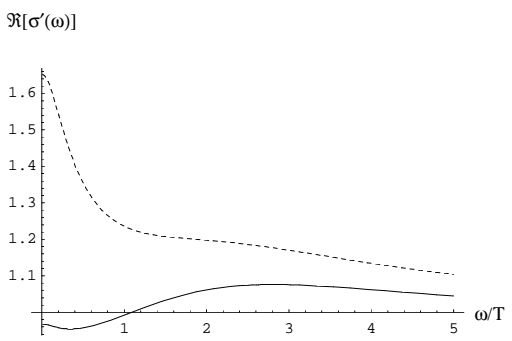

Fig. 18. The theoretical dependence 199 of the real part of the conductivity, normalized by the Drude normal conductivity, on $\omega / T, \Re\left[\sigma^{\prime}(\omega)\right]=\operatorname{Re}[\sigma(\omega)] / \sigma^{\mathrm{n}}$. The dashed line refers to the $a b$-plane component of the conductivity tensor whose Drude normal conductivity is $\sigma_{\|}^{\mathrm{n}}=N(0) e^{2} \tau v_{F}^{2}$. The solid line refers to the $c$-axis component whose Drude normal conductivity is $\sigma_{\perp}^{\mathrm{n}}=\sigma_{\|}^{\mathrm{n}} J^{2} s^{2} / v_{F}^{2}$. In this plot we have put $T \tau=0.3, E_{F} / T=50, r=0.01, \epsilon=0.04, T \tau_{\varphi}=4$.

invariable. With a further increase of frequency $\min \left\{T-T_{c}, \tau_{\varphi}^{-1}\right\} \lesssim \omega$ the $\mathrm{AL}$ and MT contributions decay; $\operatorname{Re} \sigma_{\perp}$ remains negative up to $\omega \sim \min \left\{T, \tau^{-1}\right\}$, then it changes its sign at $\omega \sim \tau^{-1}$, reaches maximum and rapidly decreases. The following high frequency behavior is governed by the Drude law. So one can see that the characteristic pseudo-gap-like behavior in the frequency dependence of the c-axis optical conductivity takes place: a transparency window appears in the range $\omega \in\left[T-T_{c}, \tau^{-1}\right]$.

In the case of the $a b$-plane optical conductivity the two first positive contributions are not suppressed by the interlayer transparency, and exceed considerably the negative DOS contribution in a wide range of frequencies. Any pseudo-gap like behavior is therefore unlikely in $\sigma_{x x}^{\text {tot }}(\omega)$ : the reflectivity will be of the metallic kind.

\subsection{Thermoelectric power above the superconducting transition [203,201}

Thermoelectric effects are difficult both to calculate and to measure if compared with electrical transport properties. At the heart of the problem lies the fact that the thermoelectric coefficients in metals are the small resultant of two opposing currents which almost completely cancel. In calculating the thermoelectric power one finds that the electrons above the Fermi level carry a heat current that is nearly the negative of that carried by the electrons below $E_{F}$. In the model of a monovalent metal in which band structure and scattering probabilities are symmetric about $E_{F}$, this cancellation would be exact; in a real metal a small asymmetry survives. Because of their compensated nature, thermoelectric effects are very sensitive to the characteristics 
of the electronic spectrum, presence of impurities and peculiarities of scattering mechanisms. The inclusion of many-body effects, such as electron-phonon renormalization, multi-phonon scattering, drag effect, adds even more complexity to the problem of calculating the thermoelectric power. Among such effects, there is also the influence of thermodynamical fluctuations on the thermoelectric transport in a superconductor above the critical temperature. This problem has been attracting the attention of theoreticians for more than twenty years, since the paper of Maki 202 appeared, where the logarithmically divergent AL contribution was predicted for the two-dimensional case. So the AL term turns out to be less singular compared with the corresponding correction to conductivity.

In every case where the main AL and MT fluctuation corrections are suppressed for some reason, the contribution connected with fluctuation renormalization of the one-electron density of states (DOS) can become important. The analogous situation also occurs in the case of the thermoelectric coefficient 200,201. Although the DOS term has the same temperature dependence as the AL contribution 202 203, it turns out to be the leading fluctuation contribution in both the clean and dirty cases, due to its specific dependence on the electron mean free path.

We introduce the thermoelectric coefficient $\vartheta$ in the framework of linear response theory as

$$
\vartheta=\frac{1}{T} \lim _{\omega \rightarrow 0} \frac{\operatorname{Im}\left[Q^{(e h) R}(\omega)\right]}{\omega}
$$

where $Q^{(e h) R}(\omega)$ is the Fourier representation of the retarded correlation function of electric $J^{e}$ and heat $J^{h}$ current operators in Heisenberg representation:

$$
Q^{(e h) R}\left(X-X^{\prime}\right)=-\Theta\left(t-t^{\prime}\right)\left\langle\left\langle\left[J^{h}(X), J^{e}\left(X^{\prime}\right)\right]\right\rangle\right\rangle .
$$

Here $X=(\mathbf{r}, t)$ and $\langle\langle\cdots\rangle\rangle$ represents both thermodynamical averaging and averaging over random impurity positions. The correlation function $Q^{(e h) R}$ in the diagrammatic technique is represented by a bubble with two exact electron Green's functions and two external field vertices, the first, ev, associated with the electric current operator and the second, $\frac{i}{2}\left(\varepsilon_{n}+\varepsilon_{n+\nu}\right) \mathbf{v}$, associated with the heat current operator $\left(\varepsilon_{n}\right.$ is fermionic Matsubara frequency) 172. The first order fluctuation corrections to $Q^{(e h)}\left(\omega_{\nu}\right)$ are represented by the same diagrams as for conductivity (see Fig.9).

The first diagram describes the AL contribution to thermoelectric coefficient and was calculated in [202,203] with the electron-hole asymmetry factor taken into account in the fluctuation propagator. Diagrams 2-4 represent the Maki-Thompson contribution, neither anomalous nor regular parts of these diagrams contribute to $\vartheta$ in any order of electron-hole asymmetry 172,203. The contribution from diagrams 5-10 describes the correction to $\vartheta$ due to fluctuation renormalization of the one-electron density of states. Evaluating 
it in the same way as (193) but with one heat current vertex one obtains a vanishing result if electron-hole asymmetry is not taken into account. The first possible source of this factor is contained in the fluctuation propagator; it was used in 203] for the AL diagram but for the DOS contribution this correction results in non-singular contributions to $\vartheta$ only and can be neglected. Another source of electron-hole asymmetry is connected with expansion of energy-dependent functions in powers of $\xi / E_{F}$ near the Fermi level:

$$
\nu(\xi) \mathbf{v}^{2}(\xi)=\nu(0) \mathbf{v}^{2}(0)+\xi\left[\frac{\partial\left(\nu(\xi) \mathbf{v}^{2}(\xi)\right)}{\partial \xi}\right]_{\xi=0} .
$$

Only the second term in Eq. (256) contributes to the thermoelectric coefficient. Performing the integration over $\xi$, summations over fermionic frequencies and analytical continuation of the result obtained we find that the contribution to the thermoelectric coefficient associated with the DOS renormalization takes the form

$$
\vartheta_{2 \mathrm{D}}^{\mathrm{DOS}}=\frac{1}{4 \pi^{2}} \frac{e T_{c}}{\nu(0) \mathbf{v}_{F}^{2}}\left[\frac{\partial\left(\nu \mathbf{v}^{2}\right)}{\partial \xi}\right]_{\xi=0} \kappa^{*}(T \tau) \ln \left[\frac{2}{\sqrt{\epsilon}+\sqrt{\epsilon+r}}\right],
$$

where

$$
\begin{aligned}
\kappa^{*}(T \tau) & =-\frac{1+\frac{\pi}{8 T \tau}}{T \tau\left[\psi\left(\frac{1}{2}+\frac{1}{4 \pi T \tau}\right)-\psi\left(\frac{1}{2}\right)-\frac{1}{4 \pi T \tau} \psi^{\prime}\left(\frac{1}{2}\right)\right]} \\
& =\left\{\begin{array}{c}
\frac{8 \pi^{2}}{7 \zeta(3)} T \tau \approx 9,4 T \tau T \tau \gg 1 \\
(T \tau)^{-1} \quad T \tau \ll 1
\end{array}\right.
\end{aligned}
$$

Summing Eq. 257) with the AL contribution 203 one can find the total correction to the thermoelectric coefficient in the case of a $2 \mathrm{D}$ superconducting film of thickness $d$ :

$$
\frac{\vartheta^{D O S}+\vartheta^{A L}}{\vartheta_{0}}=-0.17 \frac{1}{E_{F} \tau} \frac{1}{p_{F} d} \ln \left(\frac{T_{c}}{T-T_{c}}\right)\left[\kappa^{*}\left(T_{c} \tau\right)+5.3 \ln \frac{\omega_{D}}{T_{c}}\right],
$$

Assuming $\ln \left(\omega_{D} / T_{c}\right) \approx 2$ one finds that the DOS contribution dominates the $\mathrm{AL}$ one for any value of impurity concentration: $\kappa^{*}$ has a minimum at $T \tau \approx 0.3$ and even at this point the DOS term is twice as large. In both limiting cases $T \tau \ll 1$ and $T \tau \gg 1$ this difference strongly increases.

In practice, although the Seebeck coefficient $S=-\vartheta / \sigma$ is probably the easiest to measure among the thermal transport coefficients, the comparison between experiment and theory is complicated by the fact that $S$ cannot be calculated directly; it is rather a composite quantity of the electrical conductivity and thermoelectric coefficient. As both $\vartheta$ and $\sigma$ have corrections due to superconducting fluctuations, the total correction to the Seebeck coefficient is given by

$$
\Delta S=S_{0}\left(\frac{\Delta \vartheta}{\vartheta_{0}}-\frac{\Delta \sigma}{\sigma_{0}}\right)
$$


We see that the fluctuations result in a decrease of the absolute value of the overall Seebeck coefficient as the temperature approaches $T_{c}$.

The situation is complicated additionally in HTS materials, where the temperature behavior of the background value of the thermoelectric power remains unknown. This does not permit to extract precisely from the experimental data the fluctuation part $\Delta \vartheta$ to compare it with the theoretical prediction. Nevertheless the very sharp maximum in the Seebeck coefficient experimentally observed in a few papers [204,205,206 seems to be unrelated to the fluctuation effects. This conclusion is supported by recent analysis of the temperature dependence of the thermoelectric coefficient close to the transition in Refs. 207.

\subsection{The effect of fluctuations on NMR characteristics 176}

Preliminaries. In this Section we discuss the contribution of superconducting fluctuations to the spin susceptibility and the NMR relaxation rate. For both these effects the interplay of different fluctuation contributions is unusual with respect to the case of the conductivity. Like in the case of the optical conductivity, the fluctuation contributions to the spin susceptibility and the NMR relaxation rate can manifest themselves as the opening of a pseudogap already in the normal phase, a phenomenon which is characteristic to HTS compounds.

We begin with the dynamic spin susceptibility $\chi_{ \pm}^{(R)}(\mathbf{k}, \omega)=\chi_{ \pm}\left(\mathbf{k}, i \omega_{\nu} \rightarrow\right.$ $\omega+i 0^{+}$) where

$$
\chi_{ \pm}\left(\mathbf{k}, \omega_{\nu}\right)=\int_{0}^{1 / T} d \tau e^{i \omega_{\nu} \tau}\left\langle\left\langle\hat{T}_{\tau}\left(\hat{S}_{+}(\mathbf{k}, \tau) \hat{S}_{-}(-\mathbf{k}, 0)\right)\right\rangle\right\rangle .
$$

Here $\hat{S}_{ \pm}$are the spin raising and lowering operators, $\hat{T}_{\tau}$ is the time ordering operator, and the brackets denote thermal and impurity averaging in the usual way. The uniform, static spin susceptibility is given by $\chi_{s}=\chi_{ \pm}^{(R)}(\mathbf{k} \rightarrow$ $0, \omega=0$ ) while the dynamic NMR relaxation rate is given by

$$
\frac{1}{T_{1} T}=\lim _{\omega \rightarrow 0} \frac{A}{\omega} \int \frac{d^{3} \mathbf{k}}{(2 \pi)^{3}} \operatorname{Im} \chi_{ \pm}^{(R)}(\mathbf{k}, \omega)
$$

where $A$ is a positive constant involving the gyromagnetic ratio.

For non-interacting electrons $\chi_{ \pm}^{0}\left(\mathbf{k}, \omega_{\nu}\right)$ is determined by the usual loop diagram. Simple calculations lead to the well known results for $T \ll E_{F}$ : $\chi_{s}^{0}=\nu$ (Pauli susceptibility) and $\left(1 / T_{1} T\right)^{0}=A \pi \nu^{2}$ (Korringa relaxation). We will present the fluctuation contributions in a dimensionless form by normalizing to the above results.

To leading order in $G i$ the fluctuation contributions to $\chi_{ \pm}$can be discussed with the help of the same diagrams drawn for the conductivity in Fig. 9. It is important to note that the role of the external vertices (electron interaction with the external field) is now played by the $\hat{S}_{ \pm}(\mathbf{k}, \tau)$ operators. This means 
that the two fermion lines attached to the external vertex must have opposite spin labels (up and down). Consequently, the Aslamazov-Larkin diagram for $\chi_{ \pm}$does not exist since one cannot consistently assign a spin label to the central fermion for spin-singlet pairing. The next set of diagrams to consider is the Maki-Thompson contribution. While the MT diagrams for $\chi_{ \pm}$appear to be identical to those for the conductivity, there is an important difference in topology which arises from their spin structure. It is easy to see, by drawing the fluctuation propagator explicitly as a ladder of attractive interaction lines, that the MT diagram is a non-planar graph with a single fermion loop. In contrast the MT graph for the conductivity is planar and has two fermion loops. The number of loops, in accordance with the rules of diagrammatic technique [92], affects the sign of the contribution.

The diagrams 5 and 6 represent the effect of fluctuations on the singleparticle self energy, leading to a decrease in the DOS. The DOS diagrams 7 and 8 include impurity vertex corrections (note that these have only a single impurity scattering line as additional impurity scattering in the form of a ladder has a vanishing effect). Finally 9 and 10 are the DOS diagrams with the Cooperon impurity corrections.

Spin Susceptibility [208, 176]. We note that, when the external frequency and momentum can be set to zero at the outset, as is the case for $\chi_{s}$, there is no anomalous MT piece (which as we shall see below is the most singular contribution to $1 / T_{1}$ ). The MT diagram 2 then yields a result which is identical to the sum of the DOS diagrams 5 and 6 .

In the clean limit $\left(T_{c} \tau \gg 1\right)$ the fluctuation contribution is given by $\chi_{s}^{\mathrm{fl}}=\chi_{s 2}+\chi_{s 5}+\chi_{s 6}$; all other diagrams turn out to be negligible. In the dirty case $\left(T_{c} \tau \ll 1\right)$, the DOS diagrams 5 and 6 , together with the regular part of the MT diagram (2), yield the same result as in the clean limit (of the order $\left.\mathcal{O}\left(T_{c} / E_{F}\right)\right)$. One can see, that this contribution is negligible in comparison with the expected dominant one for the dirty case of the order $\mathcal{O}\left(1 / E_{F} \tau\right)$. A thorough study of all diagrams shows that the important graphs in the dirty case are those with the Cooperon impurity corrections MT 3 and 4, and the DOS ones 9 and 10. This is the unique example known to us where the Cooperons, which play a central role in the weak localization theory, give the leading order result in the study of superconducting fluctuations. Diagrams 3 and 4 give one half of the final result given below; diagrams 9 and 10 provide the other half. The total fluctuation susceptibility is $\chi_{s}^{\mathrm{f}}=$ $\chi_{s 3}+\chi_{s 4}+\chi_{s 9}+\chi_{s 10}$. Interesting, that in both the clean and dirty cases $\chi_{s}^{\mathrm{fl}} / \chi_{s}{ }^{(0)}$ can be expressed by the same formula if one expresses the coefficient in terms of the GL number $G i_{(2)}(176)$ :

$$
\frac{\chi_{s}^{\mathrm{fl}}}{\chi_{s}{ }^{(0)}}=-2 G i_{(2)} \ln \left(\frac{2}{\sqrt{\epsilon}+\sqrt{\epsilon+r}}\right) \text {. }
$$

It is tempting to explain the negative sign of the fluctuation contribution to the spin susceptibility in Eq. (262) as arising from a suppression of the DOS 
at the Fermi level. But one must keep in mind that only the contribution of diagrams 5 and 6 can strictly be interpreted in this manner; the MT graphs and the coherent impurity scattering described by the Cooperons do not permit such a simple interpretation.

Relaxation Rate[209, 210,204, 176]. The calculation of the fluctuation contribution to $1 / T_{1}$ requires rather more care than $\chi_{s}$ because of the subtleties of analytic continuation. Let us define the local susceptibility

$$
K\left(\omega_{\nu}\right)=\int(d \mathbf{k}) \chi_{+-}\left(\mathbf{k}, \omega_{\nu}\right) .
$$

In order to write down the fluctuation contribution to $1 / T_{1}$ for the case of an arbitrary impurity concentration including the ultra-clean case let us start from the anomalous MT contribution and evaluate it using the standard contour integration techniques

$$
\begin{aligned}
\lim _{\omega \rightarrow 0} \frac{1}{\omega} \operatorname{Im} K^{(a n) R}(\omega)=-\frac{\pi \nu^{2}}{8} \int(d \mathbf{q}) L(\mathbf{q}, 0) \mathcal{K}(\mathbf{q}) \\
\mathcal{K}(\mathbf{q})=2 \tau \int_{-\infty}^{\infty} \frac{d z}{\cosh ^{2}(z / 4 T \tau)} \frac{1}{\left(\sqrt{l^{2} q^{2}-(z-i)^{2}}-1\right)} \times \\
\frac{1}{\left(\sqrt{l^{2} q^{2}-(z+i)^{2}}-1\right)} .
\end{aligned}
$$

We have used the impurity vertices in the general form (233). The first simple limiting case for (264) is $l q \ll 1$, when the square roots in the denominator can be expanded and $\mathcal{K}(\mathbf{q})=2 \pi / \mathbf{D} q^{2}$. As we already know from Section 8.4 this corresponds to the usual local approximation and covers the domain $T_{c} \tau \ll 1 / \sqrt{\epsilon}$. Introducing the pair breaking rate $\gamma_{\varphi}$ as an infrared cut off one can find:

$$
\frac{\delta\left(1 / T_{1}\right)^{M T(a n)}}{\left(1 / T_{1}\right)^{0}}=\frac{28 \zeta(3)}{\pi^{4}} G i_{(2, d)} \frac{1}{\epsilon-\gamma_{\varphi}} \ln \left(\epsilon / \gamma_{\varphi}\right) .
$$

The other limiting case is the "ultra-clean limit" when the characteristic $q$ values satisfy $l q \gg 1$. This is obtained when $T \tau \gg 1 / \sqrt{\epsilon} \gg 1$. From (264) we then find $\mathcal{K}(\mathbf{q})=4 \ln (l q) / v q$, which leads to

$$
\frac{\delta\left(1 / T_{1}\right)^{M T(a n)}}{\left(1 / T_{1}\right)^{0}}=\frac{\pi^{3}}{\sqrt{14 \zeta(3)}} G i_{(2, c l)} \frac{1}{\sqrt{\varepsilon}} \ln (T \tau \sqrt{\epsilon}) .
$$

We note that in all cases the anomalous MT contribution leads to an enhancement of the NMR relaxation rate over the normal state Korringa value. In particular, the superconducting fluctuations above $T_{c}$ have the opposite 
sign to the effect for $T \ll T_{c}$ (where $1 / T_{1}$ drops exponentially with $T$ ). One might argue that the enhancement of $1 / T_{1}$ is a precursor to the coherence peak just below $T_{c}$. Although the physics of the Hebel-Slichter peak (pile-up of the DOS just above gap edge and coherence factors) appears to be quite different from that embodied in the MT process, we note that both effects are suppressed by strong inelastic scattering.

We now discuss the DOS and the regular MT contributions which are important when strong dephasing suppresses the anomalous MT contribution discussed above. The local susceptibility arising from diagrams 5 and 6 can be easily evaluated. The other remaining contribution is from the regular part of the MT diagram. It can be shown that this regular contribution is exactly one half of the total DOS contribution from diagrams 5 and 6 . All other diagrams either vanish (as is the case for graphs 7 and 8) or contribute at higher order in $1 / E_{F} \tau$ (this applies to the graphs with the Cooperon corrections). The final results can be presented in a unique way for the clean (but not ultra-clean) and dirty cases by means of the $G i_{(2)}$ number :

$$
\frac{\delta\left(1 / T_{1}\right)^{D O S}}{\left(1 / T_{1}\right)^{0}}=-12 G i_{(2)} \ln \left(\frac{2}{\sqrt{\epsilon}+\sqrt{\epsilon+r}}\right) .
$$

The negative DOS contribution to the NMR relaxation rate is evident from the Korringa formula and it sign seems very natural while the sign of the positive Maki-Thompson contribution can generate a questions about its physical origin. Let us consider a self-intersecting trajectory and the motion of the electron along it with fixed spin orientation (let us say "spin up"). If, after passing a full turn, the electron interacts with the nucleus and changes its spin state and momentum to the opposite value it can pass again along the previous trajectory moving in the opposite direction. Interaction of the electron with itself on the previous stage of the motion is possible due to the retarded character of the Cooper interaction and such a pairing process, in contrast to the AL one, turns out to be an effective mechanism for relaxation near $T_{c}$. This purely quantum process opens a new mechanism of spin relaxation, and so contributes positively to the relaxation rate $1 / T_{1}$.

In the case of the nuclear magnetic relaxation rate calculations, the electron interaction causing nuclear spin flip is considered. If one would try to imagine an $\mathrm{AL}$ process of this type he would be in trouble, because the electron-nuclei scattering with spin-flip evidently transforms the initial singlet state of the fluctuation Cooper pair in a triplet-one, which is forbidden in the scheme discussed. So the formally discovered absence of the AL contribution to the relaxation rate is evident enough.

It is worth mentioning that the cancellation of the MT and DOS contributions to conductivity found in Section 8.4 is crucial for the fluctuation contributions to the NMR relaxation rate. In fact, the MT and DOS contributions here have the same structure as in the conductivity while the $\mathrm{AL}$ contribution is absent. So the full fluctuation correction to the NMR relaxation rate in clean superconductor simply disappears. 
Discussion. The main results of this Section, valid for $\epsilon \ll 1$, can be summarized as follows:

(1) Fluctuations lead to a suppression of the spin susceptibility $\chi_{s}$, due to the combined effect of the reduction of the single particle density of states arising from the self energy contributions, and of the regular part of the MT process.

(2) "Cooperon" impurity interference terms, involving impurity ladders in the particle-particle channel, are crucial for the $\chi_{s}$ suppression in the dirty limit.

(3) The processes which dominate the results in (1) and (2) above have usually been ignored in fluctuation calculations (conductivity, $1 / T_{1}$, etc.). The spin susceptibility is unusual in that the AL and the anomalous MT terms, which usually dominate, are absent.

(4) For weak pair-breaking $\left(1 / \tau_{\varphi} \ll T_{c}\right)$, an enhancement of $1 / T_{1} T$, coming from the positive anomalous MT term, takes place [210,176].

(5) Strong dephasing suppresses the anomalous MT contribution, and $1 / T_{1}$ is then dominated by the less singular DOS and the regular MT terms. Being negative, these contributions lead to a suppression of spectral weight and a decrease in $1 / T_{1}$.

An intensive controversy took place in recent years in relation to the magnetic field dependence of the fluctuation contribution to $1 / T_{1}$. The situation here resembles much the situation with the magnetoconductivity: a positive MT contribution is suppressed by the magnetic field while the magnetic field dependent part of the DOS contribution increases with the growth of the field. But in contrast to the magnetoconductivity, which can be measured extremely precisely, the NMR relaxation rate measurements are much more sophisticated. The result of this delicate competition, depending on many parameters $\left(r, \gamma_{\varphi}, \tau\right)$, was found in HTS materials to be qualitatively different in experiments of various groups. The absence of a strong positive AL contribution, possible d-pairing, killing the MT contribution [210], small magnitude of the sum of MT and DOS effects even in the case of s-pairing, lack of the precise values of $r, \gamma_{\varphi}, \tau$, leading to contradictive theoretical predictions 176, 113, 180,212, the dispersion in the quality of samples and experimental methods were the reason of this discussion 113, 213,214,215, 44, 216.

\section{Conclusions}

Several comments should be made in conclusion. As was mentioned in the Introduction the first "fluctuation boom" took place at the end of 60's beginning of 70's, just after the discovery of the fluctuation smearing of the superconducting transition and formulation of the microscopic theory of fluctuations. The discovery of HTS reanimated this interest and, in order to account for the specifics of these layered structures with high critical tempera- 
tures, low charge carrier concentration and other particularities, considerable progress in studies of fluctuation phenomena was achieved (see for instance the conference proceedings [217,218 and the extensive review article [76]). As it is recognized now the optimally or overdoped phases of HTS compounds present an example of a "bad" Fermi liquid. The accounting for superconducting fluctuations is identical to including of the electron-electron interaction beyond the Fermi-liquid approximation. As a result a lot of anomalies of the normal state properties of such HTS compounds can be explained. The situation was found to be much more sophisticated in underdoped phases where the quasiparticle approach, which from we have started this Chapter, fails.

In the fluctuation theory discussed above, as in modern statistical physics in general, two methods have been used mainly: they are the diagrammatic technique and the method of functional (continual) integration over the order parameter. Each of them as we have seen, has its own advantages and disadvantages and in different parts of this review we used the former or the latter.

The years of the fluctuation boom coincided with the maximum development of the diagrammatic methods of many body theory in Condensed Matter Theory. This methods turns out to be extremely powerful: any physical problem, after its clear formulation and writing down the Hamiltonian, can be reduced to the summation of some classes of diagrams. The diagrammatic technique is especially comfortable for problems containing some small parameter. In the theory of superconducting fluctuations such a small parameter exists: as we have seen, it is the Ginzburg-Levanyuk number $G i_{(D)}$ which is expressed as some powers of the ratio $\max \left\{T_{c}, \tau^{-1}\right\} / E_{F}$. This is why superconducting fluctuations led to the appearance of the small corrections to different physical values in a wide range of temperatures, and due to this smallness these corrections can be evaluated quantitatively. On the other hand their specific dependence on nearness to the critical temperature $T-T_{c}$ permits to separate them in experiment from other effects.

In those cases when fluctuations are small it is possible to restrict their summation to the ladder approximation only. The diagrammatic technique permits in a unique way to describe the quantum and classical fluctuations, the thermodynamical and transport effects

In the description of thermodynamic fluctuations the method of functional integration turned out to be simpler. The ladder approximation in the diagrammatic approach is equivalent to the Gaussian approximation in functional integration. The method of functional integration turns out to be more effective too in the case of strong fluctuations, for instance, in the immediate vicinity of the phase transition. The final equations of the renormalization group carried out by means of functional integrations turn out to be equivalent to the result of the summation of the parquet diagrams series. Nevertheless the former derivation is much more simple. 
There is one another reason why we have tried to use both methods and even to carry out some results in both ways. In its explosive development of the last decades physics became an "oral science". In the process of such direct communication near a blackboard it is difficult to write and to read some cumbersome formulas. The language of diagrams is much more comprehensive: by drawing them the speaker demonstrates that this one is small and that one has to be taken into account for this and that reasons clear for the experienced listener. The success of the diagrammatic technique in some sense is similar to the success of geometry in Ancient Greece, where the science was "oral" too.

This advantage of the diagrammatic technique transforms into its disadvantage when there is no direct communication between the speaker and listener. It is difficult to learn the diagrammatic technique by a textbook on your own, when no one helps you to find the necessary insight on a complex graph. May be because of similar reasons geometry disappeared in Middle Ages when direct communications between scientists was minimal while the "written" algebra had continued to develop. Operating with Osvald Spengler "prosymbols" we can say that the diagrammatic technique belongs more to the Ancient Greece culture style with its "finite body" prosymbol, while functional integration, side by side with the Vikings travels to unknown lands and Leibnitz analysis of infinitesimals, is an evident modern contribution to West-European culture with its "infinite space" prosymbol.

That is why, suspecting that the modern physics in the near future can fall down to a "New Middle Ages" period, we have carried out some results by means of functional integration instead of the diagrammatic technique.

\section{Acknowledgments}

In the first place we would like to express our deep gratitude to R.S. Thompson and T. Mishonov, who were the first readers of the manuscript and made a lot of valuable comments. We are grateful to our colleagues and friends G. Balestrino, A. Buzdin, F. Federici, V. Galitski, D. Geshkenbein, A. Koshelev, D. Livanov, Yu.N. Ovchinnikov, A. Rigamonti, G. Savona, collaboration and discussions with whom helped us in writing this work. A.A.Varlamov acknowledges the financial support of COFIN-MURST 2000 and the Scientific Exchange Programme of the University of Minnesota. A considerable part of this work was written during the visits in their frameworks. A.I. Larkin acknowledges the financial support of the NSF Grant No. DRM-9812340.

\section{References}

1. V.L.Ginzburg, L.D.Landau, Zh. Exp. E Teor. Fiz. 20, 1064 (1950). 
2. J.Bardeen, L.N.Cooper, J.R.Schrieffer, Phys.Rev. 106, 162; 108, 1175; (1957).

3. V.L.Ginzburg, Soviet Solid State 2, 61 (1960).

4. A.P.Levanyuk, Zh. Exp. ES Teor. Fiz. 36, 810 (1959).

5. L.G.Aslamazov, A.I.Larkin, Soviet Solid State 10, 875 (1968); Phys. Letters 26A, 238 (1968).

6. K.Maki, Progr. Theor. Phys. (Kyoto) 39, 897 (1968); 40, 193 (1968).

7. R.S.Thompson, Phys. Rev. B1, 327 (1970).

8. R.E.Glover, Phys. Rev. Lett. A25, 542 (1967); J. Low Temp. Phys. 6, (1970).

9. K.Maki, in Superconductivity, edited by R.D.Parks (Dekker, New York), (1969).

10. L.P.Gorkov, Zh. Exp. \& Teor. Fiz. 37, 1407 (1959).

11. V.V.Schmidt, in Proceedings of the 10th International Conference on Low Temperature Physics, (VINITI, Moscow), C2, 205 (1967).

12. Tsuboi Taketumi, Suzuki Takao, Journ. of Phys. Soc. of Japan 42, (1977).

13. A.I.Larkin, Ann.Phys.(Leipzig) 8, 785 (1999).

14. A.I. Larkin, Yu. N. Ovchinnikov. Zh. Exp. E Teor. Fiz. 119, 595 (2001); condmat/0010014 (2000).

15. L.D.Landau, A.A.Abrikosov, I.M.Khalatnikov, DAN USSR. 95, 437, ibid.773, ibid 1177, (1954).

16. I.T.Dyatlov, V.V. Sudakov, K.A.Ter-Martirosyan, Zh. Exp. E Teor. Fiz. 32, 767 (1957).

17. V.V.Sudakov, DAN USSR. 111, 338 (1956).

18. A.I.Larkin, D.E.Khmelnitski, Zh. Exp. \& Teor. Fiz. 56, 2087 (1969).

19. M.Gel Man, F.E.Low, Phys.Rev. 95, 1300 (1954).

20. E.S.Stueckelberg, A.Peterman, Helv. Phys. Acta 24, 153 (1951).

21. C. Di Castro, G.Jona-Lasinio, Physics Lett. A29, 322 (1969).

22. K.G.Wilson, Phys.Rev. B4, 3174, ibid. 3184(1971).

23. A.S.Patashinski, V.L.Pokrovski, The Fluctuation Theory of Phase Transitions, Nauka, Moscow (1982).

24. A.A.Varlamov, L.Yu, Phys.Rev. 44, 7078 (1991).

25. T.Tsuzuki, Journ. Low Temp. Phys. 9, 525 (1972).

26. D.E.Prober, M.R.Beasley, R.E.Schwall, Phys. Rev. B15, 5245 (1977).

27. W.E.Lawrence and S.Doniach, Proceedings of the Twelfth International Conference on Low Temperature Physics, edited by E.Kanda, Academic Press of Japan, Kyoto, p.361 (1971).

28. K.Yamaji, Phys. Lett. A38, 43 (1972).

29. L.G.Aslamazov and A.I.Larkin, Zh. Exp. ES Teor. Fiz. 67, 647 (1973).

30. A.Schmid, Phys. Rev. 180, 527 (1969).

31. H.Schmidt, Z. Phys. B216, 336 (1968).

32. R.E.Prange, Phys. Rev. B1, 2349 (1970).

33. B.R.Patton, V.Ambegaokar, J.W.Wilkins, Solid St.Commun. 7, 1287, (1969).

34. J.Kurkijarvi, V.Ambegaokar and G.Eilenberger, Phys. Rev. B5, 868 (1972).

35. J.P. Gollub, M.R.Beasley, R.S.Newbower, M.Tinkham, Phys. Rev.Lett. 22, 1288 (1969).

36. J.P. Gollub, M.R.Beasley, M.Tinkham, Phys. Rev.Lett. 25, 1646 (1970).

37. J.P. Gollub, M.R.Beasley, R.Callarotti, M.Tinkham, Phys. Rev. B7, 3039 (1973).

38. P.A.Lee, S.R.Shenoy, Phys. Rev.Lett. 28, 1025 (1972).

39. S.P.Farrant, C.E.Gough, Phys. Rev.Lett. 34, 943 (1975). 
40. W.J.Skocpol and M.Tinkham, Rep.Prog. Phys. 38, 1094 (1975).

41. R.S.Thompson, V.Z.Kresin, Mod. Phys. Lett. B2, 1159 (1988).

42. K.F.Quader, E.Abrahams, Phys. Rev. B38, 11977 (1988).

43. W.C.Lee, R.A.Klemm, D.C.Johnson, Phys. Rev.Lett. 63, 1012 (1989).

44. P.Carretta, A.Lascialfari, A.Rigamonti, A.Rosso, A.Varlamov, Phys. Rev. B61, 12420 (2000).

45. T.M.Mishonov, E.S.Penev, Thermodynamics of Gaussian Fluctuations and paraconductivity in layered superconductors. International Journal of Modern Physics 14, 3831 (2000) (cond-mat N 0004023).

46. A.Buzdin, V.Dorin in "Fluctuation Phenomena in High Temperature Superconductors", ed. by M.Ausloos and A.A.Varlamov, NATO-ASI Series, Kluwer, Dordrecht (1997).

47. T.Mishonov, in "Physics and material Science of High Temperature Superconductors ", NATO ASI E 181, Edited by R.Kossovski et al., (Kluwer, Dordrecht, 1990).

48. A.Koshelev, Phys. Rev. B50, 506 (1994).

49. R.A.Klemm, M.R.Beasley, A.Luther, Phys. Rev. B8, 5072 (1973).

50. S.Hikami, A.Fujita, A.I. Larkin, Phys. Rev. B44, 10400 (1991).

51. C.Baraduc, A.Buzdin, J-Y. Henry, J.P.Brison and L.Puech, Physica C248, 138 (1995).

52. A.Junod, J-Y.Genoud, G.Triscone, Physica C294, 115 (1998).

53. A.Lascialfari, A.Rigamonti, P.Tedesco, to be published (2001).

54. A.I.Buzdin, B.Vuyichich, Mod. Physics Letters B4, 485 (1990).

55. L.Glazman, A.Koshelev, Soviet JETP 70, 774 (1990); A.Koshelev, private communication, (1999).

56. V.S.Berezinskii, Zh. Exp. ES Teor. Fiz. Letters 34, 610 (1971).

57. J.M.Kosterlitz and P.J.Thouless, J. Phys. C6, 1181 (1973).

58. D.R.Nelson, J.M.Kosterlitz, Phys. Rev.Lett. 39, 1201 (1977).

59. P.C.Hohenberg, Phys. Rev. 158, 383 (1967).

60. P.Minnhagen, Rev. Mod. Phys. 59, 1001 (1987).

61. P.Minnhagen in "Fluctuation Phenomena in High Temperature Superconductors", ed. by M.Ausloos and A.A.Varlamov, NATO-ASI Series, Kluwer (1997).

62. V.Vaks, A.Larkin, Soviet JETP 22, 678 (1966).

63. W.A.Little, Phys. Rev. 166, 398 (1967).

64. J.S.Langer, V.Ambegaokar, Phys. Rev. 164, 498 (1967).

65. B.I.Halperin, T.C.Lubenski, Shang-keng Ma, Phys. Rev.Lett. 32, 292 (1974).

66. A. Schmid. Phys. Konden. Mater. 5, 302 (1966).

67. C.Caroli, K. Maki, Phys. Rev. 159, 306,316 (1967).

68. E. Abrahams, T. Tsuneto, Phys. Rev. 152, 416 (1966).

69. J.W.F.Woo, E. Abrahams, Phys. Rev. 169, 407 (1968).

70. C. Di Castro, W.Young, Il Nuovo Cimento B62, 273 (1969).

71. S.Ullah, A.T.Dorsey, Phys. Rev. B44, 262 (1991).

72. L.P. Gor'kov, G.M. Eliashberg. Zh. Exp. ES Teor. Fiz. 54, 612 (1968).

73. L.G.Aslamazov, A.I.Larkin, Proceedings of the "1st European conference on condesed matter" European Phys.Soc, Geneva, Switzerland (1971).

74. T.Mishonov, A.Pozheninnikova, J.Indekeu, to be published (2001).

75. L.D.Landau, E.M.Lifshitz, Course of Theoretical Physics, vol.3, "Quantum Mechanics ", Pergamon Press, Oxford, (1978).

76. A.A.Varlamov, G.Balestrino, D.Livanov, E.Milani, Advances in Physics 48, 655 (1999). 
77. K.B.Efetov, Zh. Exp. \& Teor. Fiz. 76, 1781 (1979).

78. G.Blatter, V.B.Geshkenbein, A.I.Larkin, Phys. Rev. Lett. 68, 875 (1992).

79. P.W.Anderson, J. Phys. Chem. Sol. 11, 26 (1959).

80. A.A.Abrikosov, L.P.Gorkov, Soviet JETP 8, 1090; 9, 220 (1959).

81. A.Kapitulnik and G.Kotliar, Phys. Rev. Lett. 54, 474 (1985).

82. P.Wollhardt and D. Wolfle, "Anderson localization ", edited by Y. Nagaoka and H.Fukuyama, Springer Verlag, Berlin, (1982).

83. L.N.Bulaevski, A.A.Varlamov, M.V.Sadovski, Soviet Solid State Physics 28, 997 (1986).

84. M.P.A.Fisher, Phys. Rev. Lett. 65, 923 (1990).

85. Yu.N.Ovchinnikov, Soviet JETP 36, 366 (1973).

86. S.Maekawa and H.Fukuyama, Journ. of Phys. Soc. of Japan 51,1380 (1982); H.Takagi and Y.Kuroda, Solid State Commun. 41, 643 (1982).

87. A.A.Varlamov, V.V.Dorin, Sov. JETP 64, 1159 (1986).

88. A.M.Finkelstein, Soviet JETP Letters 45, 37 (1987).

89. A.Goldman and N.Markovic, Physics Today, November 1998 and refs.inside.

90. G.M.Eliashberg, Soviet JETP 11, 696 (1960).

91. B.N.Narozhny, Soviet JETP 77, 301 (1993); Phys. Rev. B49, 6375 (1994).

92. A.A.Abrikosov, L.P.Gorkov, I.E.Dzyaloshinski, "Methods of the quantum field theory in statistical physics ", Dover Publications, New York (1963).

93. E.Abrahams, M.Redi, and C.Woo, Phys. Rev. B1, 218 (1970).

94. C.Di Castro, C.Castellani, R.Raimondi, A.Varlamov, Phys. Rev. B49, 10211 (1990).

95. L.B.Ioffe, A.I.Larkin, A.A.Varlamov, L.Yu, Phys. Rev. B47, 8936 (1993); Physica C 235-240, 1963 (1994).

96. K.E.Gray, D.H.Kim, Phys. Rev. Lett. 70, 1693 (1993).

97. V.V.Dorin, R.A.Klemm, A.A.Varlamov, A.I.Buzdin, D.V.Livanov, Phys. Rev. B48, 12951 (1993).

98. L.G.Aslamazov, A.A.Varlamov, Journ. of Low Temp. Phys. 38, 223 (1980).

99. B.L.Altshuler, M.Yu.Reizer and A.A.Varlamov, Soviet JETP 57, 1329 (1983).

100. A.I.Larkin, D.E.Khmelnitski, Soviet Phys. Uspekhi, 136, 536 (1982).

101. W.Breing, M.C.Chang, E.Abrahams, P.Wolfle, Phys.Rev. B 31, 7001 (1985).

102. A.A.Abrikosov, Fundamentals of the Theory of Metals, North-Holland, Elsevier, Groningen (1988).

103. R.A.Klemm and S. H.Liu, Phys. Rev. B49, 6375 (1994).

104. S. H.Liu and R.A.Klemm Phys. Rev. B48, 4080; ibid 10650 (1993).

105. B.L.Altshuler and A.G.Aronov, Electron-Electron Interaction in Disordered Conductors in Efros, A.L. and Pollak, M. (eds.), Elseiver Scientific Publishing (1985).

106. G.M.Eliashberg, Zh. Exp. E Teor. Fiz. 41, 1241 (1961).

107. R.A.Klemm, J. Low Temp. Phys. 16, 381 (1974).

108. C.Baraduc, V.Pagnon, A.Buzdin, J.Henry, C.Ayache, Phys. Lett. A166, 267 (1992).

109. S.Ami, K.Maki, Phys. Rev. B18, 4714 (1978).

110. B.R.Patton, Phys. Rev. Lett. 27, 1273 (1971).

111. J.Keller, V Korenman, Phys. Rev. Lett. 27, 1270 (1971); Phys. Rev. B5, 4367 (1972).

112. S.Yip, Phys. Rev. B41, 2612 (1990).

113. P.Carretta, A.Rigamonti, A.A.Varlamov, D.V.Livanov, Phys. Rev. B54, R9682 (1996). 
114. A.Varlamov, Europhysics Letters 28, 347 (1994).

115. M. Reizer, Phys. Rev. B45, 12949 (1992).

116. M.Ausloos, Ch.Laurent, Phys. Rev. B37, 611 (1988).

117. P.P.Frietas, C.C.Tsuei, T.S.Plaskett, Phys. Rev. B36, 833 (1987).

118. M.Hikita, M.Suzuki, Phys. Rev. B41, 834 (1990).

119. M.Akinaga, D.Abukay, L.Rinderer, Mod. Phys. Lett. 2, 891 (1988).

120. A.Poddar, P.Mandal, A.N.Das, B.Ghosh, P.Choudhury, Physica C159 , 231 (1989).

121. D.H.Kim, A.M.Goldman, J.H.Kang, K.E.Gray, R.T.Kampwirth, Phys. Rev. B39, 12275 (1989).

122. G.Balestrino, A.Nigro, R.Vaglio, Phys. Rev. B39, 12264 (1989).

123. G.Kumm, K.Winzer, Physica B165-166 , 1361 (1990).

124. M.R.Cimberle, C.Ferdeghini, D.Marrè, M.Putti, S.Siri, F.Federici, A.Varlamov, Phys. Rev. B55, R14745 (1997).

125. L.Reggiani, R.Vaglio, A.Varlamov, Phys. Rev. B44, 9541 (1991).

126. G.Balestrino, E.Milani, A.Varlamov, Physica C210 , 386 (1993).

127. G.Balestrino, E.Milani, and A.A.Varlamov, JETP Letters 61, 833 (1995).

128. Y.F.Yan, P.Matl, J.M.Harris and N.P.Ong, Phys. Rev. B52, R751 (1995) ; N.P.Ong, Y.F.Yan, and J.M.Harris, Procedings of CCAST Symposium on High Tc Superconductivity and the C60 Family, Beijing, (1994).

129. W.Lang, Physica C282-287, (1997).

130. K.Nakao, K.Takamaku, K.Hashimoto, N.Koshizuka and S.Tanaka, Physica B201, 262 (1994).

131. K.Hashimoto, K.Nakao, H.Kado and N.Koshizuka, Phys. Rev. B53, 892 (1996).

132. G.Heine, W.Lang, X.L.Wang and X.Z.Wang, J. Low Temp. Phys. 105, 945 (1996).

133. T. Kimura, S. Miyasaka, H. Takagi, K. Tamasaku, H. Eisaki, S. Uchida, M. Hiroi, M. Sera, K. Kobayashi, Phys. Rev. B53, 8733 (1996).

134. J.Axnas, W.Holm, Yu.Eltsev, and O.Rapp, Phys Rev. Lett. 77, 2280 (1996).

135. A. Wahl, D. Thopart, G. Villard, A. Maignan, V. Hardy, J.C. Soret, L.Ammor, A.Ruyter, Phys. Rev. B59, 7216 (1999).

136. M.Suzuki and M. Hikita, Phys. Rev. B39, 4756 (1989); Phys. Rev. B44, 249 (1991).

137. A.Matsuda, T.Ishii, K.Kinishita, T.Yamada, Physica C162-164, 371 (1989); A.Matsuda, T.Hirai, S.Komiyama, Solid State Commun. 68, 103 (1988).

138. J.Sugawara, H.Iwasaki, N.Kobayashi, H.Yamane and T.Hirai, Phys. Rev. B46, 14818 (1992).

139. W.Holm, M.Andersson, O.Rapp, M.A.Kulikov and I.N.Makarenko, Phys. Rev. B48, 4227 (1993).

140. W.Lang, G.Heine, W.Kula and R.Sobolewski, Phys. Rev. B51, 9180 (1995).

141. W.Holm, O.Rapp, C.N.L.Johnson and U.Helmersson, Phys. Rev. B52, 3748 (1995).

142. Yu.I.Latyshev, O.Laborde and P.Monceau, Europhysics Letters 29, 495 (1995).

143. W. Lang, Z. Phys. B97, 583 (1995).

144. T.Watanabe, A.Matsuda, Physica C263, 313 (1996).

145. W.Lang, Physica C245, 69 (1995).

146. W. Volz, F.S. Razavi,G. Quiron, H.-U. Habermeier, A.L. Solovjov, Phys. Rev. B55 6631 (1997). 
147. C.Sekirnjak, W.Lang, S.Proyer, P.Schwab, Physica C243, 60 (1995).

148. S.Hikami, A.I.Larkin, Mod. Phys. Lett. B2, 693 (1988).

149. A.G.Aronov, S.Hikami and A.I.Larkin, Phys. Rev. Lett. 62, 965 (1989).

150. J.B.Bieri and K.Maki, Phys. Rev. B42, 4854 (1990).

151. J.B.Bieri, K.Maki, and R.S.Thompson, Phys. Rev. B44, 4709 (1991).

152. A.S.Nygmatulin, A.Varlamov, D.Livanov, G.Balestrino and E.Milani, Phys. Rev B53 (1996).

153. A. Wahl, D. Thopart, G. Villard, A. Maignan, Ch.Simon, J.C.Soret, L.Ammor, A.Ruyter, Phys. Rev. B60, 12495 (1999).

154. A.Buzdin, A.Varlamov, Phys. Rev. B58, 14195 (1998).

155. D.Thopart, A. Wahl, Ch.Simon, J.C.Soret, L.Ammor, A.Ruyter, A.Buzdin, A.A.Varlamov, S. de Brion, Phys. Rev. B62, 9721 (2000).

156. A.A.Abrikosov, L.P.Gorkov, Soviet JETP 11, 1781 (1960).

157. R.Ikeda, T.Ohmi, and T.Tsuneto, Journ. of Phys. Soc. of Japan Letters 58, 1377 (1989); ibid. 60, 1051 (1991).

158. D.Livanov, E.Milani, G.Balestrino and C.Aruta, Phys. Rev. B55 R8701 (1997).

159. K.Maki, Phys. Rev. Lett. 30, 648 (1973).

160. I.S.Beloborodov, K.B.Efetov, A.I.Larkin, Phys. Rev. B61, 9145 (2000).

161. L.N.Bulaevski, Zh. Exp. EG Teor. Fiz. 66, 2212(1974).

162. A.I.Larkin, Zh. Exp. ES Teor. Fiz. Letters 31, 219 (1980).

163. E.Abrahams, J. Stat. Phys. 38, 89 (1985); J.M.B. Lopes dos Santos, E.Abrahams, Phys. Rev. B31, 172 (1985).

164. M.E.Gershenzon, V.N.Gubankov, Yu.E.Zhuravlev, Solid State Commun. 45, 87 (1983).

165. R.Rosenbaum, Phys. Rev. B32, 2190 (1985).

166. N.Mori, Jpn. J. Appl. Phys. Suppl. 26, 1339 (1987).

167. B.I.Belevtsev, Yu.F.Komnik, A.V.Fomin, J. Phys. B58, 111 (1985); Fiz. Nizk. Temp. (USSR) 10, 850 (1984).

168. V.M.Galitski, A.I.Larkin, Phys. Rev. B63, 1745X (2001).

169. H.Fukuyama, H.Ebisawa, and T.Tuzuki, Prog. Theor. Phys. 46,1028 (1971).

170. E.Abrahams, R.E.Prange, and M.E.Stephen, Physica 55, 230 (1971).

171. T.Inoue, S.Miwa, K.Okamoto, M.Awano, Journ. of Phys. Soc. of Japan 46, 418 (1979).

172. A.A.Varlamov and D.V.Livanov, Soviet JETP 71, 325 (1990).

173. A.G.Aronov and A.B. Rapoport, Mod. Phys. Lett. B6,1093 (1992).

174. A.G.Aronov, S.Hikami and A.I.Larkin, Phys. Rev. B51, 3880 (1995).

175. D.V.Livanov, G.Savona, A.A.Varlamov, Phys. Rev. B62, 8675 (2000).

176. M.Randeria and A.A.Varlamov, Phys. Rev. B50, 10401 (1994).

177. I.Aleiner, L.Glazman, A.Rudin, Phys. Rev. B55, 322 (1997).

178. A.I.Larkin, Yu.A.Ovchinnikov, J. Low Temp. Phys. 10, 407 (1973).

179. A.A.Varlamov and V.V.Dorin, Soviet JETP 57, 1089 (1983).

180. M.Eschrig, D.Rainer, J.Souls, Phys.Rev. B59, 12095 (1999).

181. J.Axnaes, J. Low Temp. Phys. 117, 259 (1999).

182. R.W.Cohen, B.Abels, Phys. Rev. 168, 444 (1968).

183. B.L.Altshuler and A.G.Aronov, Soviet JETP 50, 968 (1979).

184. M.Belogolovski, O. Chernyak and A.Khachaturov, Fiz. Nizk. Temp. (USSR) 12, 630 (1986).

185. M.Park, M.S.Isaacson, J.M.Parpia, Phys. Rev. Lett. 75, 3740 (1995). 
186. H.J.Tao, Farun Lu, E.L.Wolf, Physica C 282-287, 563 (1997).

187. T.Watanabe et al., Phys. Rev. Lett. 79, 2113 (1997); A.Matsuda, S.Sugita, T.Watanabe, Phys. Rev. B60, 1377 (1999); T.Watanabe, T.Fujii, A.Matsuda, Phys. Rev. Lett. 84, 5848 (2000).

188. M.Suzuki, S.Karimoto, K.Namekawa, Journ. of Phys. Soc. of Japan 67, v.3 (1998).

189. Ch.Renner, B.Revaz, J-Y. Genoud, K.Kadowaki and O.Fischer, Phys. Rev. Lett. 80, 149 (1998).

190. A.M.Cucolo, M.Cuoco, A.A.Varlamov, Phys. Rev. Lett. 59, R11675 (1999).

191. M. Yu. Reizer, Phys. Rev. B48, 13703 (1993).

192. K.Maki, R.S.Thompson, Physica C162-164, 1441 (1989).

193. A.I. Larkin, Yu.N. Ovchinnikov. Zh. Exp. \& Teor. Fiz. 61, 2147 (1971).

194. A.A.Varlamov, V.V.Dorin and I.E.Smolyarenko, Soviet JETP 67, 2536 (1988).

195. A. Schmid, G. Schon. J. Low Temp. Phys. 20, 207 (1975).

196. A.I. Larkin, Yu. N. Ovchinnikov. Zh. Exp. \& Teor. Fiz. 68, 1916 (1975).

197. A.I. Larkin, Yu.N. Ovchinnikov. In "Nonequilibrium superconductivity". Edited by D.N. Langenberg and A.I. Larkin. Elsevier Science Publishers B.V. 1986.

198. A.I. Larkin, Yu.N. Ovchinnikov. Zh. Exp. E Teor. Fiz. 73, 299 (1977).

199. F.Federici, A.A.Varlamov, JETP Letters 64, 497 (1996); Phys. Rev. B57, 6071 (1997).

200. A.Varlamov, D.Livanov, L.Reggiani, Physics Letters A165, 369 (1992).

201. A.Varlamov, D.Livanov, F.Federici, JETP Letters 65, 182 (1997).

202. K.Maki, J. Low Temp. Phys. 14, 419 (1974).

203. M.Yu.Reizer and A.V.Sergeev, Phys. Rev. B50, 9344 (1994).

204. M.A.Howson et al., Phys. Rev. B41, 300 (1990).

205. N.V.Zavaritski, A.V.Samoilov, A.A.Yurgens, JETP Letters 55, 127 (1992).

206. S.Keshri, P.K.Barhai, Chech. J. Phys., 47, 249 (1997).

207. J.Mosqueira, J.A.Viera, F.Vidal, Physica C229, 301 (1994); J.Mosqueira, J.A.Viera, J.Masa, O.Cabeza, F.Vidal, Physica C253, 1 (1995);

208. T.Maniv, S.Alexander, J. Phys. C10, 2419 (1977).

209. T.Maniv, S.Alexander, Solid State Commun. 18, 1197 (1976).

210. K.Kuboki, H.Fukuyama, Journ. of Phys. Soc. of Japan 58, 376 (1989).

211. J.Heym, J. Low Temp. Phys. 89, 859 (1992).

212. P.Mosconi, A.Rigamonti, A.Varlamov, Applied Magnetic Resonance 19, 345 (2000).

213. V. Mitrovic et al., Phys. Rev. Lett. 82, 2784 (1999).

214. H.Zimmermann, M.Mali, M.Bankey, D.Brinkmann, Physica C185 - 189, 1145 (1991); D. Brinkmann, Appl. Magn. Resonance 8, 67 (1995).

215. K.Gorny et al., Phys. Rev. Lett. 82, 177 (1999).

216. G.Zheng et al., Phys. Rev. B60, R9947 (1999); G.Zheng et al., Phys. Rev. Lett. 85, 405 (2000).

217. M.Ausloos and A.A.Varlamov, Editors of "Fluctuation Phenomena in High Temperature Superconductors", ed. by NATO-ASI Series, Kluwer, Dordrecht (1997).

218. J.Bok, G.Deutcher, D.Pavuna, S.A.Wolf, Editors of "Gap Symmetry and Fluctuations in High Temperature Superconductors" (Plenum, London) (1998). 